\title{
Sensitivity analysis of flexible pavement performance parameters in the mechanistic-empirical design guide
}

\author{
Armando Orobio \\ West Virginia University
}

Follow this and additional works at: https://researchrepository.wvu.edu/etd

\section{Recommended Citation}

Orobio, Armando, "Sensitivity analysis of flexible pavement performance parameters in the mechanisticempirical design guide" (2010). Graduate Theses, Dissertations, and Problem Reports. 2979.

https://researchrepository.wvu.edu/etd/2979

This Dissertation is protected by copyright and/or related rights. It has been brought to you by the The Research Repository @ WVU with permission from the rights-holder(s). You are free to use this Dissertation in any way that is permitted by the copyright and related rights legislation that applies to your use. For other uses you must obtain permission from the rights-holder(s) directly, unless additional rights are indicated by a Creative Commons license in the record and/ or on the work itself. This Dissertation has been accepted for inclusion in WVU Graduate Theses, Dissertations, and Problem Reports collection by an authorized administrator of The Research Repository @ WVU.

For more information, please contact researchrepository@mail.wvu.edu. 


\title{
SENSITIVITY ANALYSIS OF FLEXIBLE PAVEMENT PERFORMANCE PARAMETERS IN THE MECHANISTIC-EMPIRICAL DESIGN GUIDE
}

\author{
Armando Orobio \\ Dissertation submitted to the \\ College of Engineering and Mineral Resources \\ at West Virginia University \\ in partial fulfillment of the requirements \\ for the degree of \\ Doctor of Philosophy \\ In \\ Civil Engineering
}

Committee members

Dr. John P. Zaniewski, Committee Chairperson

Dr. Radhey S. Sharma

Dr. Darrell Dean

Dr. Gary Winn

Dr. Indrajit Ray

Department of Civil and Environmental Engineering

Morgantown, West Virginia

2010

Keywords: Pavement Design, Flexible Pavement, Mechanistic - Empirical, Sensitivity Analysis, MEPDG, M-E PDG 


\begin{abstract}
Sensitivity Analysis of Flexible Pavement Performance Parameters in the Mechanistic-Empirical Design Guide

by

Armando Orobio
\end{abstract}

The Mechanistic-Empirical Pavement Design Guide (MEPDG) was the result of NCHRP project 1-37A. This is a mechanistic-empirical pavement design procedure which uses mechanistic and empirical models, nationally calibrated with information from several databases, especially the national Long-Term Pavement Performance (LTPP) study. This database includes data from pavements located throughout North America.

A large amount of inputs are required in order to perform pavement design with MEPDG. These may be classified into traffic loads, material properties and climate input parameters. The pavement distress mechanisms are too complex to be completely modeled without utilization of empirical data. So, calibration is required to improve the accuracy of the models for local conditions. The recommended approach for calibration includes review of the input data, sensitivity analysis, comparative studies, validation and calibration studies, the modification of the input defaults and calibration coefficients, and the verification of the national calibration by collecting a local validation database. The goal of this calibration is to verify that the performance models accurately predict pavement distress and ride quality. Unfortunately, the collection of the data either for calibration or individual designs requires numerous tests to characterize materials, and the field work for collecting the database to verify the models is laborious.

The main goal of this research is the determination of the most important parameters in the MEPDG. However, MEPDG is so complex and the input parameters are so numerous that the sensitivity analysis methodology must be carefully designed to identify the relative importance of each input variable. This research used space-filling computer experiments with Latin hypercube sampling, standardized regression coefficients, and Gaussian stochastic processes to categorize the relative importance of the flexible pavement performance parameters in MEPDG. The use of these statistical techniques allows analysis of the entire space of the input parameters. Additionally, this project studied the feasibility of this methodology for sensitivity analysis of the MEPDG. 


\section{ACKNOWLEDGMENTS}

I would like to thank Dr. John P. Zaniewski who was my advisor during my doctorate studies.

I thank Dr. Zaniewski for his continuous instruction, supervision, and support and the time he expended in this research.

I would like to thank my Advisory and Examining Committee members Dr. John P. Zaniewski, Dr Radhey S. Sharma, Dr Indrajit Ray, Dr. Darrell Deanand, and Dr. Gary Winn for their support, guidance, and critical review throughout the development of this dissertation.

I would like to express my gratitude to the School of Civil Engineering and Geomatics of University of Valle in Colombia for its support.

I extend sincere thanks to my mother, brothers, and wife for their never-ending support and encouragement throughout my years of study. 


\section{TABLE OF CONTENTS}

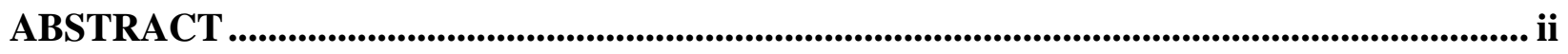

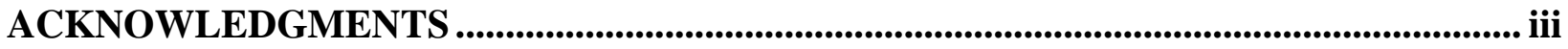

TABLE OF CONTENTS .............................................................................................................. iv

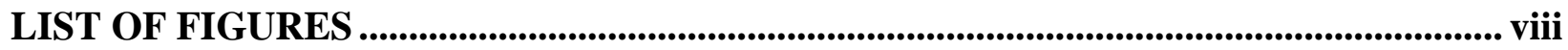

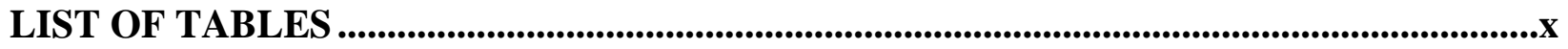

CHAPTER 1： INTRODUCTION ..................................................................................1

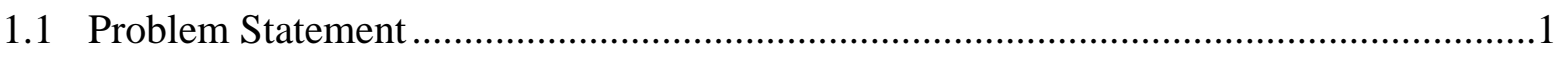

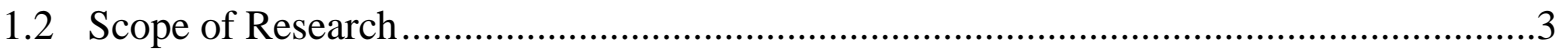

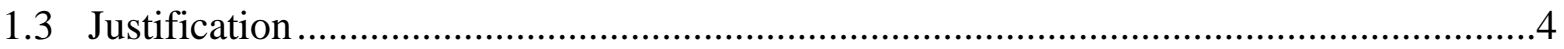

CHAPTER 2: REVIEW OF MEPDG ..........................................................................6

2.1 Guide for Mechanistic-Empirical Pavement Design ...............................................6

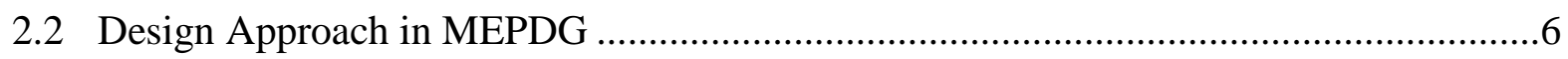

2.2.1 Hierarchical Design Inputs in MEPDG ....................................................

2.2.2 Principles of Mechanistic Procedure .......................................................10

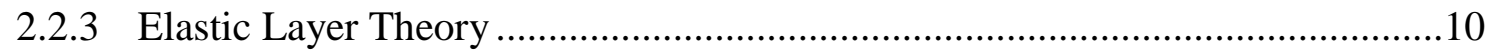

2.2.4 Advanced Material Response Models .....................................................12

2.2.5 Nonlinear Resilient Modulus Model ..............................................................12

2.2.6 Finite Elements Implementation of Nonlinear Resilient Modulus....................14

2.3 Response Models of Flexible Pavements ................................................................16

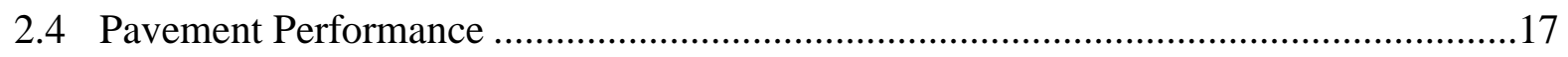

2.4.1 Pavement Performance Models ................................................................18

2.4.2 Distress Prediction Equations for Flexible Pavement .....................................19

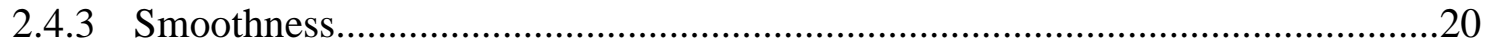

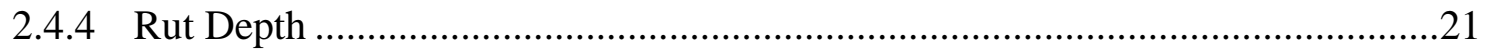

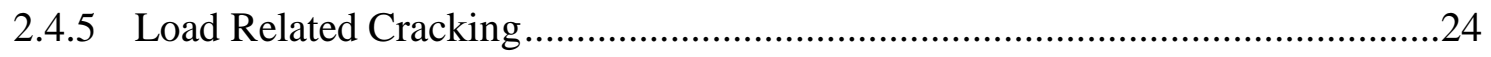


2.4.6 Fatigue Cracking for Cement Treated Bases...............................................26

2.4.7 Non-load Related Cracking ..................................................................27

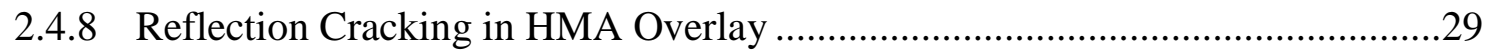

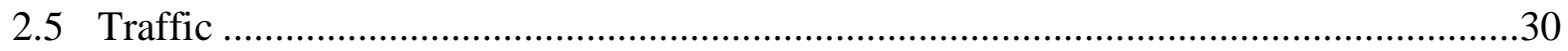

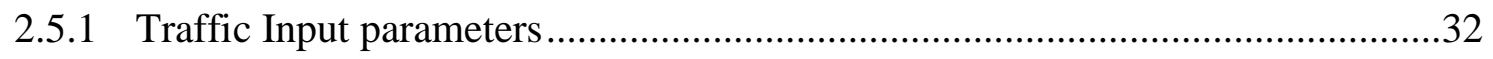

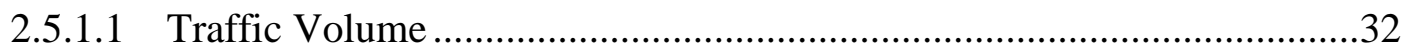

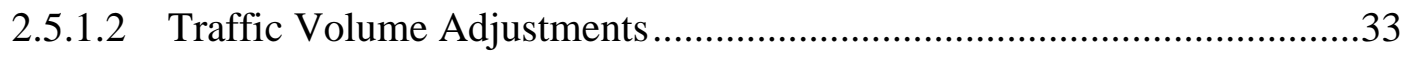

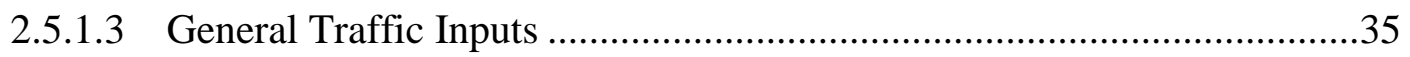

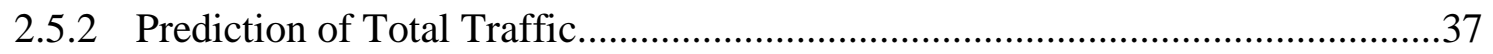

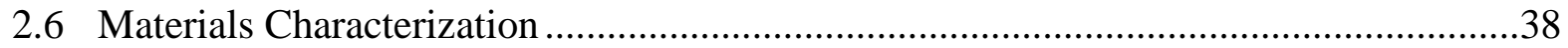

2.6.1 Hierarchical Approach for Materials Inputs...............................................39

2.6.2 Required Material Properties ............................................................ 40

CHAPTER 3： PREVIOUS SENSITIVITY ANALYSIS OF MEPDG ................................52

3.1 Pilot Study in Sampling-based Sensitivity Analysis of MEPDG ...............................52

3.2 Sensitivity of Design Input Variables in MEPDG for Rigid Pavements ......................53

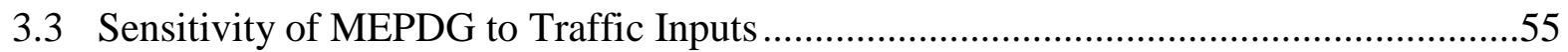

3.4 Simplified Approach for Sensitivity of Rigid Pavement Inputs in the MEPDG ...........56

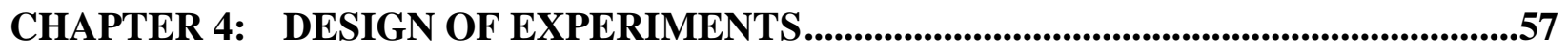

4.1 Basic Principles of Design of Experiments .........................................................58

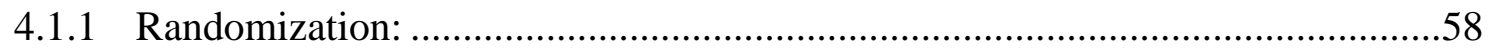

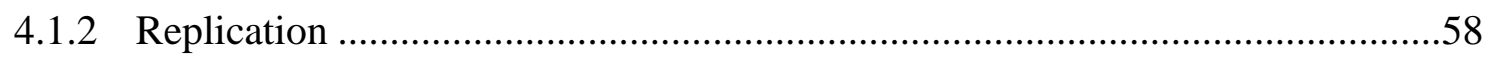

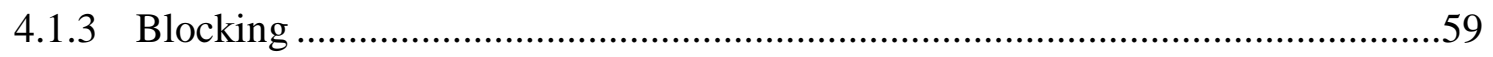

4.2 Physical Experiments and Computer Experiments ................................................59

4.2.1 Sampling in Computer Experiments .........................................................60

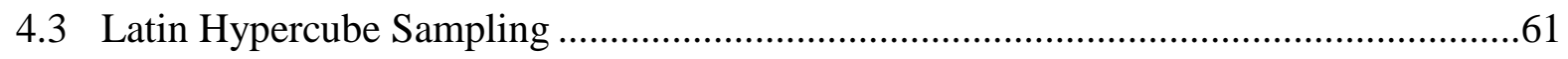

4.3.1 Latin Hypercube Sampling Algorithm ......................................................62

4.4 Sensitivity Analysis Base on Regression Analysis .............................................63 


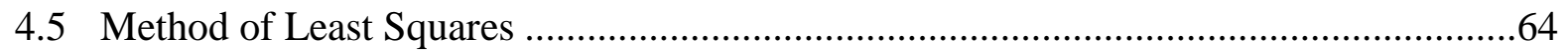

4.6 Hypothesis Testing in Regression Analysis...............................................................65

4.6.1 Test for the Significance of the Regression .......................................................65

4.6.2 Test for Individual Regression Coefficients......................................................66

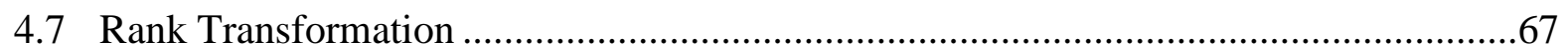

4.8 Sensitivity Based on Gaussian Stochastic Models......................................................67

CHAPTER 5: RESEARCH METHODOLOGY _................................................................69

5.1 Research Questions and Hypothesis ………………….............................................69

5.2 Research Design and Methodology ...............................................................................70

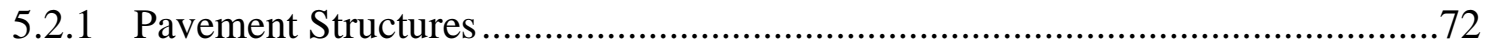

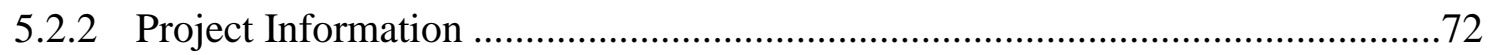

5.2.3 Input Values and Ranges in the Experiment .....................................................73

5.2.4 Analysis Process...........................................................................................73

CHAPTER 6: SENSITIVITY OF MEPDG TO TRAFFIC PARAMETERS ........................74

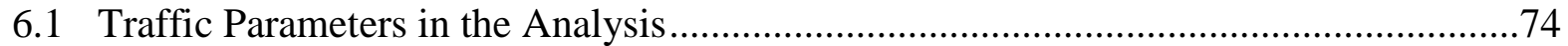

6.2 Latin Hypercube Sampling for Traffic Sensitivity Analysis ..........................................75

6.3 Results of Traffic Sensitivity of Structure 1 ..............................................................7

6.3.1 IRI Sensitivity to Traffic Input Parameters on Structure 1 ……………............77

6.3.2 Cracking Sensitivity to Traffic Input Parameters on Structure 1 ........................80

6.3.3 Rutting Sensitivity to Traffic Input Parameters on Structure 1 1..........................83

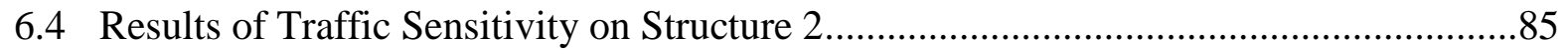

6.4.1 IRI Sensitivity to Traffic Input Parameters on Structure 2 …….........................85

6.4.2 Cracking Sensitivity to Traffic Input Parameters on Structure 2 2.......................88

6.4.3 Rutting Sensitivity to Traffic Input Parameters on Structure 2 .........................92

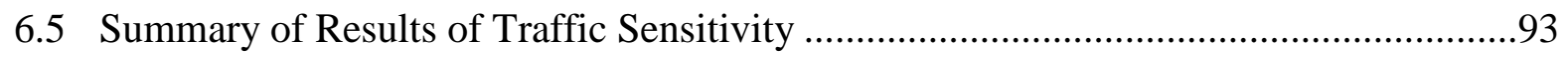

6.6 Recommended Hierarchical Levels for Traffic Input Parameters ..................................98

CHAPTER 7: SENSITIVITY OF MEPDG TO MATERIAL PROPERTIES ...................100

7.1 Material Properties Parameters in the Analysis .............................................................100 
7.2 Latin Hypercube Sampling for Material Sensitivity Analysis .................................102

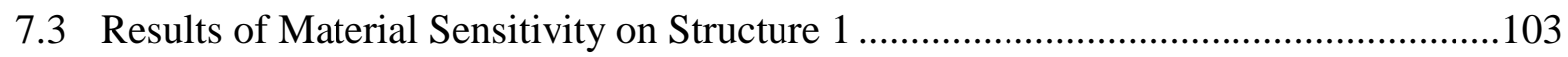

7.3.1 IRI Sensitivity to Material Input Parameters on Structure 1 ...........................103

7.3.2 Rutting Sensitivity to Material Input Parameters on Structure 1 .....................105

7.3.3 Cracking Sensitivity to Material Input Parameters on Structure 1...................107

7.4 Results of Material Sensitivity on Structure 2 .....................................................109

7.4.1 IRI Sensitivity to Material Input Parameters on Structure 2 .........................109

7.4.2 Rutting Sensitivity to Material Input Parameters on Structure 2 ....................112

7.4.3 Cracking Sensitivity to Material Input Parameters on Structure 2..................114

7.5 Summary of Results of Material Inputs Sensitivity ...............................................116

7.6 Recommended Hierarchical Levels for Material Parameters .....................................124

CHAPTER 8: MATERIAL PROPERTY DESIRABILITY ..........................................127

8.1 The Concept of Material Property Desirability …..............................................127

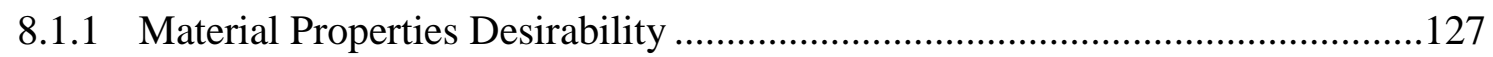

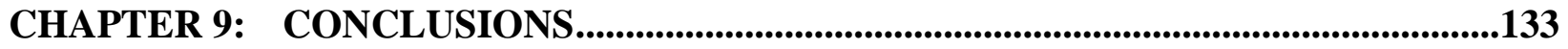




\section{LIST OF FIGURES}

FIGURE 2.1 Three-stages Design Process …………......................................................

FIGURE 2.2 Overall Design Process for Flexible Pavement ...................................................... 9

FIGURE 2.3 Multi-layered Elastic System ...................................................................... 11

FIGURE 2.4 Linear and Nonlinear Material Behavior.............................................................. 13

FIGURE 2.5 Pavement Performance Trends ……………............................................... 19

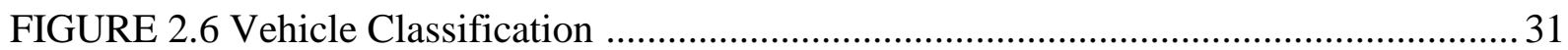

FIGURE 2.7 Interactions Between Material Properties and Models ....................................... 39

FIGURE 3.1 Pearson's and Spearman's Coefficients …………..........................................53

FIGURE 4.1 Division of the Density Function of a Variable ..................................................62

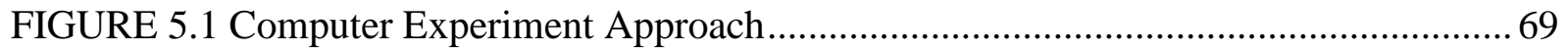

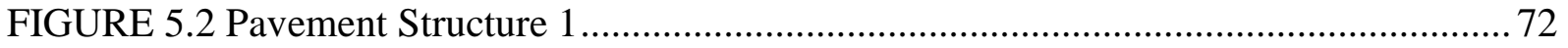

FIGURE 5.3 Pavement Structure 2 ……………………........................................... 72

FIGURE 6.1 Latin Hypercube Sampling and MEPDG Output .................................................. 75

FIGURE6.2 Pairwise Correlations Among Input Parameters ................................................. 77

FIGURE 6.3 IRI Actual by Predicted Plot for Structure 1 ..................................................... 78

FIGURE 6.4 Cracking Actual by Predicted Plot for Structure 1 ………................................. 81

FIGURE 6.5 Verification of Cracking Sensitivity by a Gaussian Process ................................ 82

FIGURE 6.6 Rutting Actual by Predicted Plot for Structure 1 ................................................ 84

FIGURE 6.7 IRI Actual by Predicted Plot for Structure 2 …................................................ 86

FIGURE 6.8 Verification of IRI Sensitivity with a Gaussian Process ...................................... 88

FIGURE 6.9 Cracking Actual by Predicted Plot for Structure 2 .............................................. 89

FIGURE 6.10 Cracking Actual by Predicted Plot for Two Approaches of Structure 2 ........... 89 
FIGURE 6.11 Verification of Cracking Sensitivity with a Gaussian Process ....................... 92

FIGURE 6.12 Rutting Actual by Predicted Plot for Structure 2 ..................................... 92

FIGURE 6.13 Standardized Regression Coefficients of Structure 1 ...................................95

FIGURE 6.14 Standardized Regression Coefficients of Structure 2 ..................................96

FIGURE 6.15 IRI Standardized Regression Coefficients............................................. 96

FIGURE 6.16 Rutting Standardized Regression Coefficients ......................................... 97

FIGURE 6.17 Cracking Standardized Regression Coefficients ....................................... 97

FIGURE 7.1 IRI Actual by Predicted Plot for Structure 1 ........................................... 103

FIGURE 7.2 Rutting Actual by Predicted Plot for Structure 1 ...................................... 106

FIGURE 7.3 Cracking Actual by Predicted Plot for Structure 1 .................................... 108

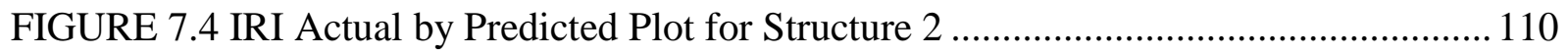

FIGURE 7.5 IRI Actual by Predicted Plot for Structure 2 .......................................... 110

FIGURE 7.6 Verification of IRI Sensitivity with a Gaussian Process .............................. 112

FIGURE 7.7 Rutting Actual by Predicted Plot for Structure 2 ........................................ 113

FIGURE 7.8 Cracking Actual by Predicted Plot for Structure 2 ................................... 115

FIGURE 7.9 Standardized Regression Coefficients for Structure 1................................ 118

FIGURE 7.10 Standardized Regression Coefficients for Structure 2 .............................. 121

FIGURE 8.1 Distribution of MEPDG Outputs ........................................................... 132

FIGURE 8.2 Distribution of MEPDG Outputs with the New Runs 11 and 181 .................. 132 


\section{LIST OF TABLES}

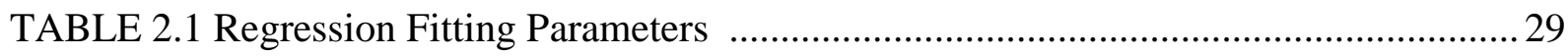

TABLE 2.2 MEPDG Traffic Growth Functions ………………………………………......... 34

TABLE 2.3 Default Values of Number of Axle Type per Truck Class .................................... 36

TABLE 2.4 Tires Width and Minimal Dual Tire Spacing ......................................................... 37

TABLE 2.5 Maximum Loads and Cold Inflation Pressures........................................................ 37

TABLE 2.6 Material Inputs for Asphalt Materials ………………………………................. 42

TABLE 2.7 Material Inputs for Chemically Stabilized Materials .............................................45

TABLE 2.8 Material Inputs for Non-stabilized Base, Subbase, and Subgrde Soils................. 48

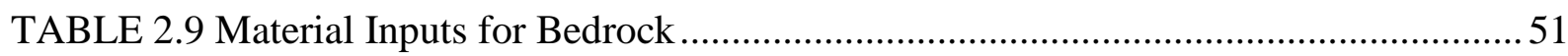

TABLE 3.1 Individual Values Utilized in Pilot Study .......................................................52

TABLE 3.2 Structural Constant Inputs in the Study …………...........................................5

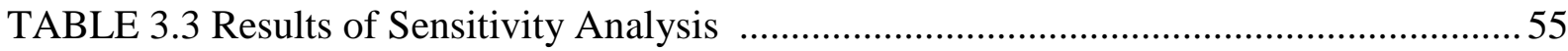

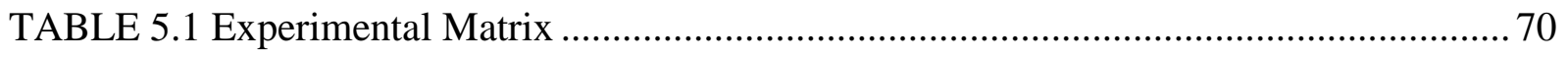

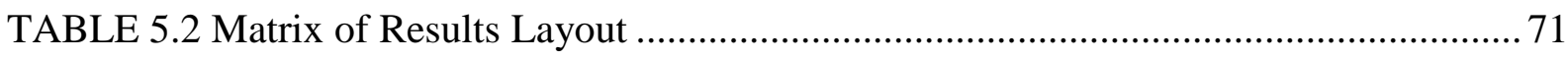

TABLE 5.3 Total Numbers of Input Parameters ………………………….......................... 73

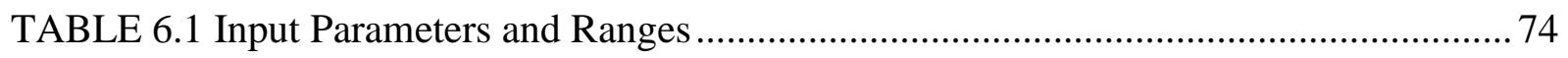

TABLE 6.2 Pairwise Correlations Coefficients Among Input Parameters ............................... 76

TABLE 6.3 IRI Regression Coefficients for Structure 1 …………………………............... 79

TABLE 6.4 IRI Standardized Regression Coefficients for Structure 1 ..................................... 80

TABLE 6.5 Cracking Regression Coefficients for Structure 1 ................................................ 82

TABLE 6.6 Cracking Standardized Regression Coefficients for Structure 1 .......................... 83

TABLE 6.7 Rutting Regression Coefficients for Structure 1 .................................................. 84 
TABLE 6.8 Rutting Standardized Regression Coefficients for Structure 1 ............................ 85

TABLE 6.9 IRI Regression Coefficients for Structure 2 …................................................ 87

TABLE 6.10 IRI Standardized Regression Coefficients for Structure 2 …............................. 87

TABLE 6.11 Cracking Regression Coefficients for Structure 2 ............................................ 90

TABLE 6.12 Cracking Standardized Regression Coefficients for Structure 2 ……................90

TABLE 6.13 Cracking Regression Coefficients for Structure 2 (Values > 5\%) ...................... 91

TABLE 6.14 Cracking Standardized Regression Coefficients for Structure 2 (> 5\%) ............ 91

TABLE 6.15 Rutting Regression Coefficients for Structure 2 …………………………........ 93

TABLE 6.16 Rutting Standardized Regression Coefficients for Structure 2 ........................... 93

TABLE 6.17 Summary of SRC from Traffic Results.............................................................. 94

TABLE 6.18 Recommended Hierarchical Levels for Traffic Inputs ...................................... 99

TABLE 7.1 Input Parameters and Ranges for Structure 1.................................................. 101

TABLE 7.2 Input Parameters and Ranges for Structure 2 …………………………......... 102

TABLE 7.3 IRI Standardized Regression Coefficients for Structure 1 ................................. 104

TABLE 7.4 Rutting Standardized Regression Coefficients for Structure 1 .......................... 107

TABLE 7.5 Cracking Standardized Regression Coefficients for Structure 1 ....................... 109

TABLE 7.6 IRI Standardized Regression Coefficients for Structure 2 …............................. 111

TABLE 7.7 Rutting Standardized Regression Coefficients for Structure 2 …....................... 114

TABLE 7.8 Cracking Standardized Regression Coefficients for Structure 2 …................... 115

TABLE 7.9 Standardized Regression Coefficients of Structure 1 ........................................117

TABLE 7.10 Standardized Regression Coefficients of Structure 2 ……………………...... 120

TABLE 7.11 Effect of Material Input Parameters in MEPDG Outputs on Structure 1 ......... 123

TABLE 7.12 Effect of Material Input Parameters in MEPDG Outputs on Structure 2 ......... 124

TABLE 7.13 Recommended Hierarchical Levels for Material Parameters for Structure 1 ... 125 
TABLE 7.14 Recommended Hierarchical Levels for Material Parameters for Structure 2 ... 126

TABLE 8.1 Material Properties Desirability for Structure 1............................................... 128

TABLE 8.2 Checking Runs 11 and 181 of Structure 1 ..................................................... 130 


\section{CHAPTER 1: INTRODUCTION}

Pavement design theory has evolved from empirical to the mechanistic-empirical (M-E) methodologies. The M-E method has been a significant advance in pavement engineering since it connects the design theories with the performance of the pavement structures in the field. This characteristic requires calibration and validation processes which require database building, field data collection technologies, and adequate technology for material and climate characterization. M-E pavement design methods are complex and require computer software which considers the factors and their interactions involved in the design procedure. Generally, structure and material properties, climate, traffic, construction, and maintenance are the factors included in the method.

\subsection{Problem Statement}

NCHRP project 1-37A, Development of the 2002 Guide for Design of New and Rehabilitated Pavement Structures, was completed in 2004 (1). The MechanisticEmpirical Pavement Design Guide (MEPDG) resulted from this project (2). This mechanistic-empirical pavement design procedure uses mechanistic and empirical models, nationally calibrated with information from several databases, especially the national Long-Term Pavement Performance (LTPP) study (1). This database includes data from pavements located throughout North America.

The software included in the guide is an important tool in the design procedure. Pavement response and pavement performance models were incorporated in the software. An iterative process is used for the design of a pavement structure. The performance of a trial section is analyzed over time using response and performance models; the results are compared to the performance criteria; the trial structure is modified if it does not meet criteria. The process is repeated until all criteria are met.

A large number of inputs are required in order to perform a MEPDG design. These may be classified into traffic loads, material properties and climate input parameters. The pavement design procedure itself has a large amount of uncertainty in the determination of input parameters. The most obvious source of 
variability is the prediction of traffic loads over the time (1). Construction process and material characteristics are also important sources of variability. Design reliability was incorporated in the guide for dealing with this uncertainty.

Three hierarchical input levels are considered in the MEPDG; Level 1 requires the direct measurement of the parameters, Level 2 allows the estimation of the parameters from regression equations or correlations, and Level 3 uses typical values. These levels classify the inputs base on the knowledge which the designers have of each parameter. Level 1 is the most accurate and Level 3 is the least accurate.

Pavement designers are encouraged to calibrate the models to local conditions in order to successfully implement the procedure. The distress mechanisms are too complex to be completely modeled without utilization of empirical data. Calibration is required to improve the accuracy of the models for local conditions. The recommended approach for calibration includes review of the input data, sensitivity analysis, comparative studies, validation and calibration studies, and the modification of the input defaults and calibration coefficients as needed (1). Calibration to local conditions includes verification of the national calibration by collecting a local validation database. The goal of this calibration is to verify that the performance models accurately predict pavement distress and ride quality. Collecting the data either for calibration or individual designs requires numerous tests to characterize materials. The field work for collecting the database for verifying the models is also laborious.

The primary interest in this research was the identification of the most sensitive input parameters of the design procedure. However, MEPDG is so complex and the input parameters are so numerous that the sensitivity analysis methodology was carefully designed to identify the relative importance of each input variable. The sensitivity analysis will be useful during the MEPDG implementation process. For example, data gathering can be done more efficiently focusing on the most sensitive parameters. The most sensitive parameter would desirably require Level 1 evaluation while the less sensitive parameters can be left at Level 2 or 3. 


\subsection{Scope of Research}

The complexity of the design procedure with several response and performance models and the large number of input parameters required for a specific design lead to uncertainty in the design process. It is necessary to perform sensitivity analysis of the input parameters for the global understanding of the MEPDG. The present work considers Latin Hypercube Sampling in order to analyze the effect of the entire input parameter space in the sensitivity analysis. It will allow the classification of the input parameter in order of importance. The main goal of this research is the determination of the most important parameters in the MEPDG. This will be helpful not only in the implementation but also in the daily use of the design procedure. Additionally, this project will study the feasibility of this methodology for sensitivity analysis of the MEPDG.

DOTs could apply this methodology to identify particular needs in their states. Once the sensitive parameters have been identified, DOTs working in the implementation or calibration of the MEPDG can focus their effort and funds on those parameters that are the most sensitive. The effort in characterization of material properties and traffic data collection can be focused on those more sensitive parameters. The local calibration factors would be more accurate and the process for the development of those factors would be less expensive and time consuming. The selection of the level for each parameter would be done with the knowledge of the impact of each parameter in the entire MEPDG.

MEPDG can be used for new or rehabilitation design for either rigid or flexible pavements. The research reported herein is limited to the design of new flexible pavements. The methodology is applicable to other design situations by altering the selection of input parameters.

MEPDG software has the ability to consider non-linear behavior through the use of finite element analysis. However, this approach has not been calibrated so it was not consider in this research. 


\subsection{Justification}

In the case of the Mechanistic-Empirical Pavement Design Guide the pavement response models are mechanistic and the pavement performance models are empirical. This characteristic makes calibration to local conditions an important step in the implementation process.

The literature review showed that a large number of input parameters are required not only for the regular use of the MEPDG but also for the calibration process. Some of the model variables and material properties are controllable or easy to be collected but others are difficult to obtain, requiring sophisticated equipment or field data from local databases which in some cases are not available or accurate. There are three hierarchical levels of design inputs, Level 1, Level 2, and Level 3, being Level 1 is the most rigorous and level 3 least rigorous; this approach is applied to traffic, materials, and environmental inputs. Inputs from the three levels may be used for a specific project.

These characteristics in MEPDG make the global understanding of the several parameters in the design procedure important for implementation of the method. Testing the sensitivity of input parameters will be helpful in the implementation, calibration process, and daily use. As shown in the literature review, several researchers have addressed sensitivity analyses of MEPDG. Most researchers have used the technique of varying the parameters one at time while leaving the others parameters unchanged. This technique does not account for parameter interactions which are an important concern in MEPDG sensitivity. Another approach followed in previous research has been the factorial experiment based on analysis of variance (ANOVA). The problem with factorial experiment is that MEPDG has a large number of parameters and parameter combinations, making a complete analysis of MEPDG with factorial experiments untractable because of the size of experiment required. Additionally, ANOVA techniques are base on the analysis of the variability due to experimental error. Since MEPDG is computer code, any time it is run with the same set of input parameters, the same output is obtained. So, there is no experimental error and ANOVA may not be suitable for this type of study. The literature review also reported a recent study using Monte Carlo simulation in a very limited range of parameters using Pearson's and Spearman's 
coefficients to analyze the sensitivity of the outputs in MEPDG. Although, this was a limited study the effectiveness of this type of random sampling was proved to work well in the case of the MEPDG. The difference between Monte Carlo and Latin Hypercube sampling is that Monte Carlo is random sampling while Latin Hypercube is a stratified random sampling which does a better job in space filling experiments to sample the entire space of the parameters with a smaller sample size.

Finding a suitable methodology for sensitivity analysis of MEPDG might have a beneficial impact in the design procedure itself. The methodology proposed may be included into the software of MEPDG as a sensitivity analysis tool integrated to the design procedure. This integration would be a useful enhancement to reduce uncertainty and address calibration and implementation of MEPDG. 


\section{CHAPTER 2: REVIEW OF MEPDG}

The literature review is divided in three parts: Part 1 summarizes the design procedure and the performance models in the MEPDG guide, the second part, chapter 3, summarizes relevant studies related to this research, and the third part, chapter 4, explains the statistical concepts needed for this research.

\subsection{Guide for Mechanistic-Empirical Pavement Design}

The AASHO Road Test was performed in the late 1950s in Ottawa, Illinois (1). The study was addressed to evaluate the performance of pavements under traffic. The Road Test provided the basis of empirical-base procedures for pavement design. The AASHTO Guide for the Design of Pavements structures was the primary result from AASHO Road Test, issued in the early 1960s and with major modifications in 1986 and 1993 (1). In subsequent years important limitations of the road test have been identified. Some of those limitations are: the test was conducted in only one climate region, with limited traffic, only one type of vehicle per loop, one type of subgrade, and a single set of materials.

Due to the limitations of the empirical procedures, the NCHRP sponsored Project 1-37A for the development of a state-of-the-art pavement design procedure using the existing mechanistic-based models and databases. The result from this project was the 2002 Mechanistic-Empirical Pavement Design Guide (MEPDG) including a software tool for analysis and design of pavement structures.

\subsection{Design Approach in MEPDG}

The design approach in the MEPDG has three stages as shown in Figure 2.1 (1). Notice that Stage 2 analysis produces a single design but Stage 3 calls for comparing multiple alternatives, so several trials should be analyzed with Stage 2 by varying the input parameters defined in Stage 1, and then comparing them in Stage 3 in order to have the better choice. 


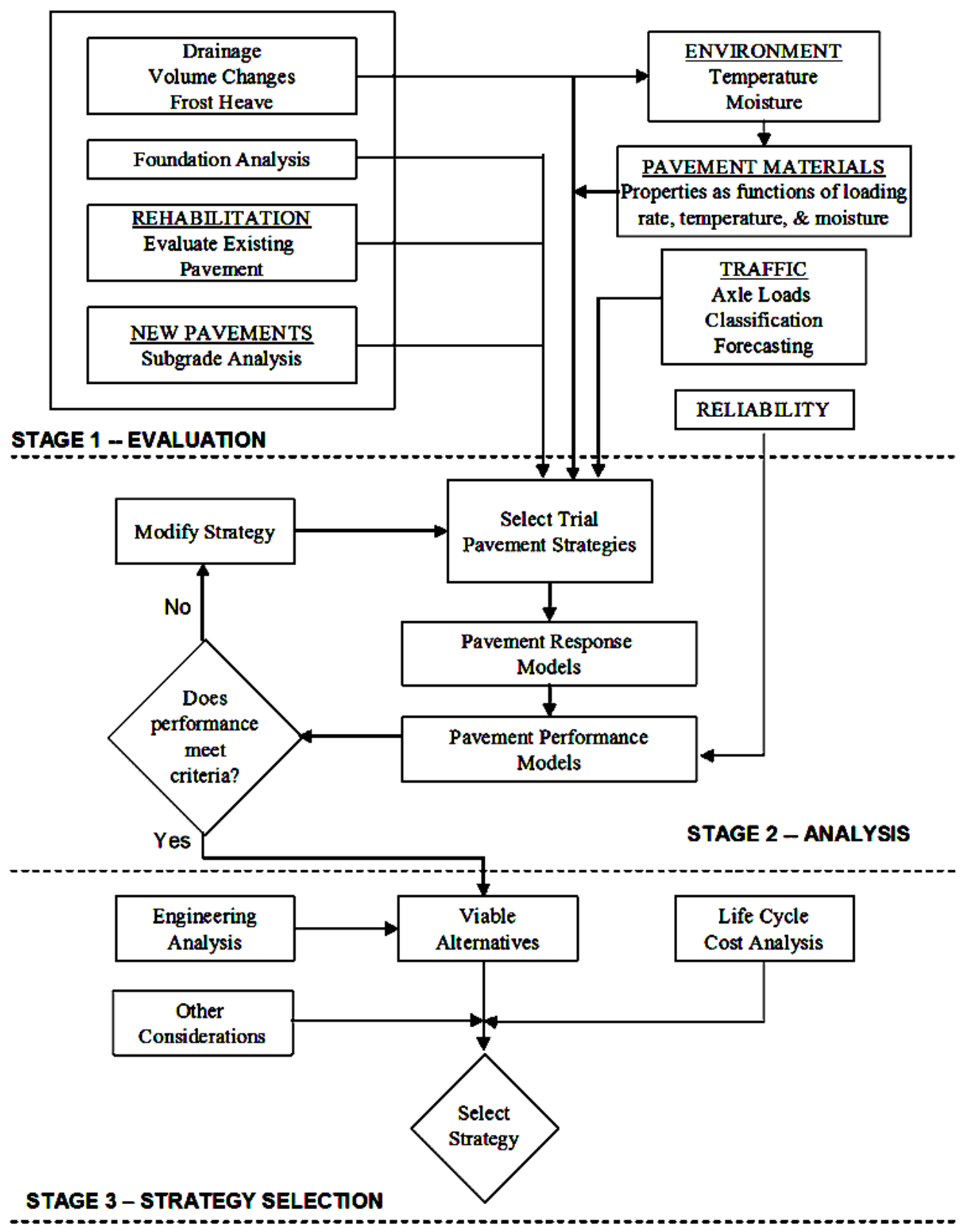

FIGURE 2.1 Three-stages Design Process (NCHRP, 2004)

- Stage 1 Development of the input values for analysis

- The input process includes four steps:

- Determination of foundation characteristics; in the case of new pavements these include stiffness, volume changes, frost heave, and thaw weakening. For 
new pavement design, subgrade improvements can be considered such as strengthening and drainage. In the case pavements of rehabilitation the foundation characteristics include evaluation of existing distress types and causes.

- Pavement materials characterization; determination of material properties for pavement response models, distress-transfer functions, and climatic models.

- Traffic input data; determination of the axle-load spectra.

- Climate considerations for modeling temperature and moisture within pavement layers using Enhance Integrated Climate Model (EICM).

\section{- Stage 2 Structural / Performance analysis}

- The analysis process includes five steps:

- Selection of the initial trial design. It can be created by the designer base on a general catalog or a previous design.

- Analysis of the trial design using the response and performance models

- Trial design is check against criteria. If the trial design does not meet the criteria it is modified and models are run again.

- The process is repeated until the trial design meets the criteria.

- The design process can be repeated using alternative design concepts.

\section{- Stage 3 Evaluation of structurally viable alternatives.}

- The set of alternative designs is evaluated for selecting the recommended design.

- Engineering and life cycle cost analysis of alternatives. Several trial designs are analyzed according to the previous stage. 
- Selection of best alternative according to the engineering and life cycle cost analysis of alternatives.

The overall design process for flexible pavement is shown in Figure 2.2 (1).

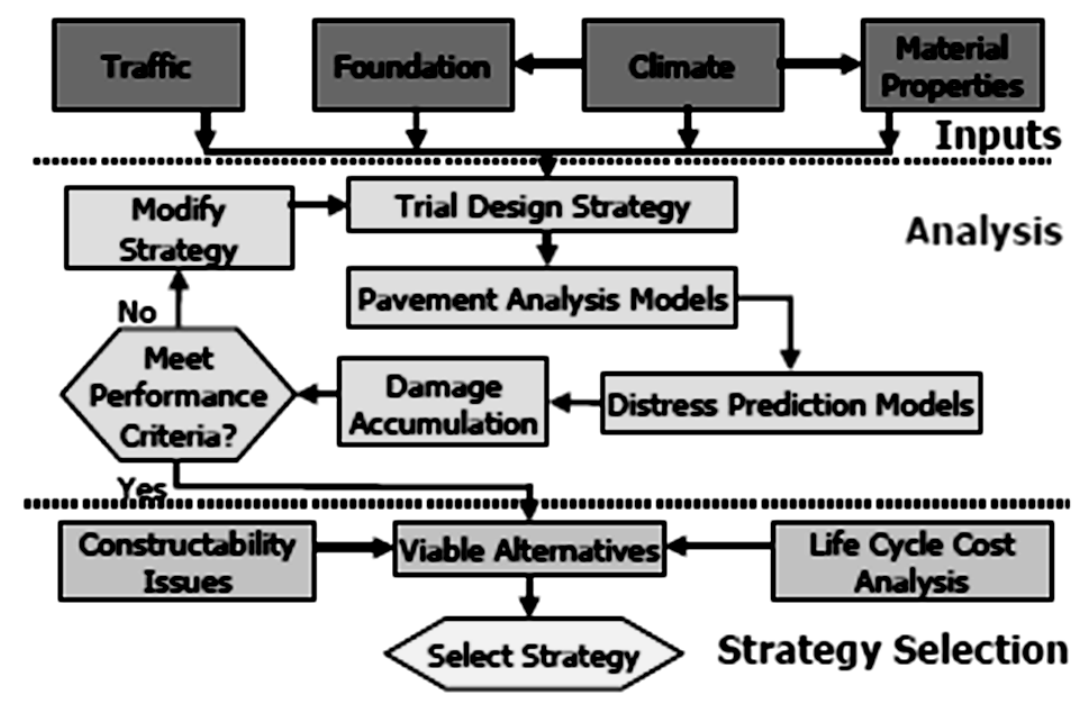

FIGURE 2.2 Overall Design Process for Flexible Pavement (NCHRP, 2004)

\subsubsection{Hierarchical Design Inputs in MEPDG}

This is a characteristic of the MEPDG that is used to categorize the designer's knowledge of the input parameters (1). This approach provides flexibility in obtaining design inputs for a project based on the criticality of the design and the available resources. Additionally, it allows agencies to use the method with little initial investment, and standard test equipment for measurement material properties. It also allows experienced users to measure many inputs for a specific project. The hierarchical approach is used with regards to traffic, material, and condition of existing pavement parameters (2). In general, three levels of input are available in MEPDG (1).

- Input Level 1: Input parameters are measured directly. This level provides the highest level of accuracy and lowest level of uncertainty or error but it has the highest test and data collection cost to determine the input values.

- Input Level 2: Input parameters are estimated from correlation or regression equation. This level provides an intermediate level of accuracy. This level can be 
used when resources or testing equipment are not available for requirements of Level 1.

- Input Level 3: Input parameters are based on typical values or regional estimates. This level provides the lowest level of accuracy.

Specific project inputs can be obtained from different levels. For example, the traffic data may be input using the Level 1 procedures if the data are available while material properties may be estimated using Level 3 if more rigorous data are not available. It is recommended that the designer use the highest level available at the time of the design. The computational models and procedures for damage, distress and smoothness are the same for all levels (2).

\subsubsection{Principles of Mechanistic Procedure}

The mechanistic analysis approach refers to the prediction of stresses, strains, and deflections in pavement layers due to traffic load and environmental conditions. A mechanistic procedure includes prediction of failure and distress parameters, estimation of material properties, and the relationship between magnitude of parameters and the failure or performance level required. The primary material properties in this theory are the elastic modulus, E, and the Poisson's ratio, $v$. Yoder and Witzack defined the general concept of a multi-layered elastic system for a flexible pavement as shown in Figure 2.3 (3).

\subsubsection{Elastic Layer Theory}

This mechanistic approach considers that a given point within any layer is under normal and shear stresses. There are three different normal stresses $\sigma_{\mathrm{z}}, \sigma_{\mathrm{r}}$, and $\sigma_{\mathrm{t}}$ acting perpendicular to the element face, where $\mathrm{z}, \mathrm{r}$, and $\mathrm{t}$ are the vertical, radial, and tangential planes respectively. There are six different shearing stresses $\tau_{\mathrm{rt}}, \tau_{\mathrm{tr}}, \tau_{\mathrm{rz}}, \tau_{\mathrm{zr}}, \tau_{\mathrm{tz}}$, and $\tau_{\mathrm{zt}}$ acting parallel to the faces. The static equilibrium conditions show that shear stresses acting on intersecting faces are equal $\tau_{\mathrm{rt}}=\tau_{\mathrm{tr}}, \tau_{\mathrm{rz}}=\tau_{\mathrm{zr}}, \tau_{\mathrm{tz}}=\tau_{\mathrm{zt}}$. At each point in the system there is an orientation of the element that the shear stresses are zero. The normal stresses under this condition are defined as principal stresses $\sigma_{1}, \sigma_{2}$, and $\sigma_{3}$. The sum of principal 
stresses is called bulk stress, $\theta$. Considering the triaxial stress state of any element, the strain may be determined as follows (3).

$$
\begin{aligned}
& \varepsilon_{z}=\left(\frac{1}{E}\right)\left[\sigma_{z}-v\left(\sigma_{r}+\sigma_{t}\right)\right] \\
& \varepsilon_{r}=\left(\frac{1}{E}\right)\left[\sigma_{r}-v\left(\sigma_{t}+\sigma_{z}\right)\right] \\
& \varepsilon_{t}=\left(\frac{1}{E}\right)\left[\sigma_{t}-v\left(\sigma_{r}+\sigma_{z}\right)\right]
\end{aligned}
$$

Where,

- $\varepsilon_{\mathrm{z}}, \varepsilon_{\mathrm{r}}, \varepsilon_{\mathrm{t}}:$ strains on the directions $\mathrm{z}, \mathrm{r}$, and $\mathrm{t}$ respectively

- $\sigma_{\mathrm{z}}, \sigma_{\mathrm{r}}, \sigma_{\mathrm{t}}$ : Principal stresses on the directions $\mathrm{z}, \mathrm{r}$, and $\mathrm{t}$ respectively

- $\quad v$ : Poisson's ratio.

- E: Elastic modulus.

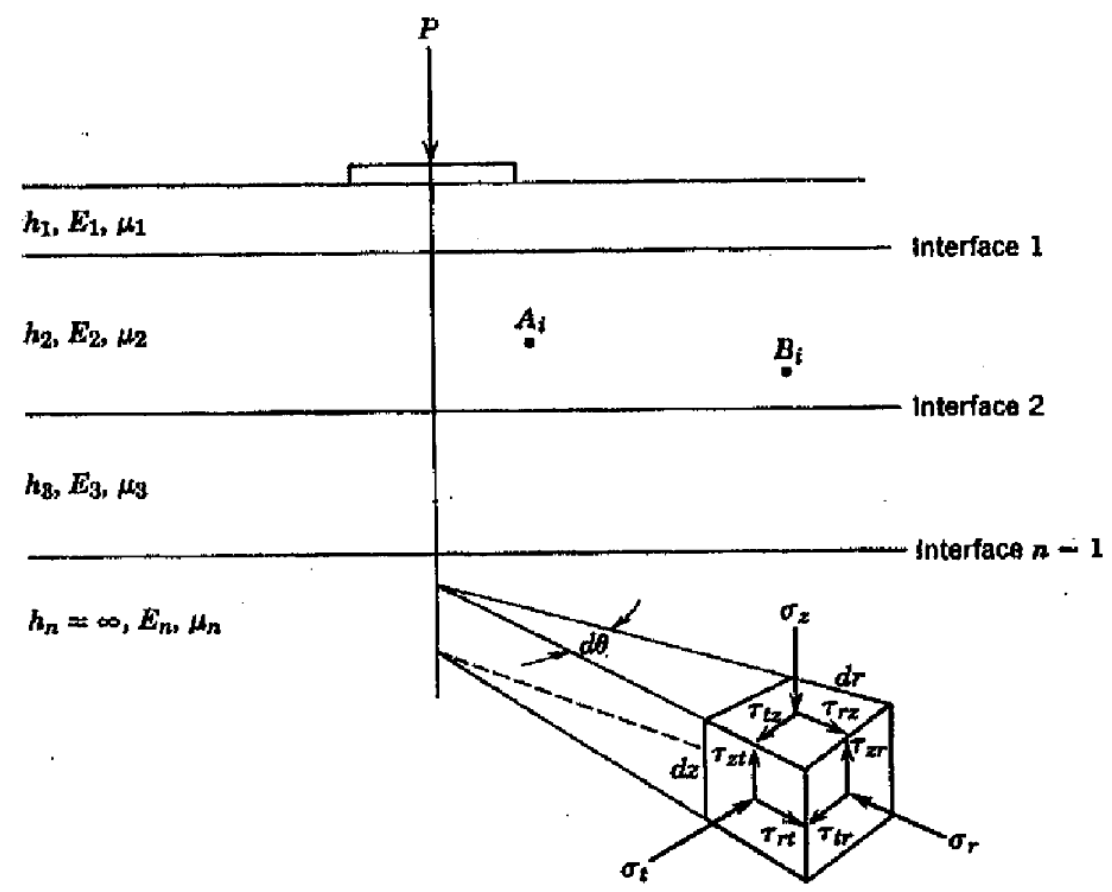

FIGURE 2.3 Multi-layered Elastic System (Yoder and Witczack, 1975) 
The multi-layer system is based on the following assumptions (4).

- Each layer is homogeneous, and isotropic.

- The layers are characterized by the modulus of elasticity, and Poisson's ratio.

- The material is weightless and horizontally infinite

- The thickness of each layer is finite and uniform.

- The subgrade is considered as an infinite layer or with rigid bottom.

- The load is uniformly applied on the surface over a circular area.

- Load is normal to the pavement surface.

- Continuity conditions are satisfied at the layer interfaces.

\subsubsection{Advanced Material Response Models}

Accurate modeling of pavement behavior needs to consider nonlinear response and viscous and viscoelastic behavior. In the case of the MEPDG the main analysis capabilities required in flexible pavement response models are (5):

- Linear material model for AC and bound, and unbound layers (lowest hierarchical level for unbound material characterization)

- Stress-dependent material model; nonlinear resilient modulus for unbound materials (highest hierarchical level for unbound material characterization)

- Quasi-static monotonically increasing loading from single or multiple wheel configurations

- Fully bonded, full slip, and intermediate interface conditions between layers.

- Viscoelastic approximation based on the time-temperature principle.

\subsubsection{Nonlinear Resilient Modulus Model}

Unbound pavement materials and asphalt concrete at high temperatures are more precisely characterized using a stress-dependent modulus. Figure 2.4 shows linear and nonlinear material behavior (5). As seen in the figure, linear behavior has a constant modulus while nonlinear behavior has stress-dependent modulus. 
Unbound materials like bases, subbases, and subgrades have nonlinear behavior; therefore, they are characterized with a stress-dependent model. The MEPDG uses the resilient modulus to estimate stiffness for the unbound layers in a pavement structure. The general model in MEPDG for calculations of resilient modulus of stress-dependent materials is indicated in equation 2.4 (5).

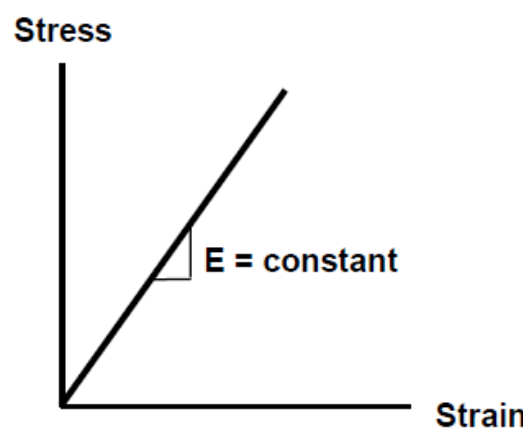

(a) Linear

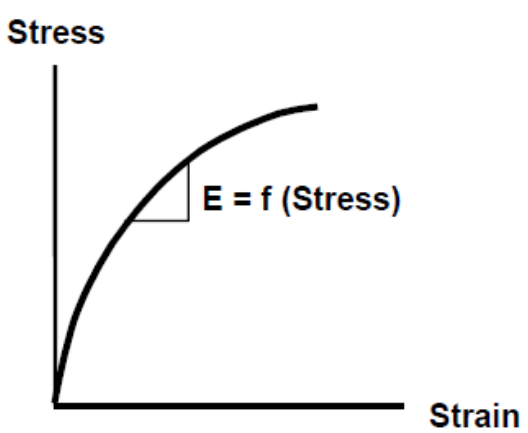

(b) Nonlinear

FIGURE 2.4 Linear and Nonlinear Material Behavior (NCHRP, 2004)

$$
M_{r}=k_{1} p_{a}\left(\frac{\theta}{p_{a}}\right)^{k_{2}}\left(\frac{\tau_{o c t}}{p_{a}}+1\right)^{k_{3}}
$$

Where,

- $\quad M_{r}=$ resilient modulus, psi.

- $\theta=$ bulk stress $=\sigma_{1}+\sigma_{2}+\sigma_{3}$

- $\sigma_{1}=$ major principal stress

- $\sigma_{2}=$ intermediate principal stress

- $\sigma_{3}=$ minor principal stress, confining pressure

- $\tau_{\text {oct }}=$ octahedral shear stress $=\frac{1}{3} \sqrt{\left(\sigma_{1}-\sigma_{2}\right)^{2}+\left(\sigma_{1}-\sigma_{3}\right)^{2}+\left(\sigma_{2}-\sigma_{3}\right)^{2}}$

- $\mathrm{p}_{\mathrm{a}}=$ normalizing stress.

- $k_{1}, k_{2}, k_{3}=$ regression constants. 


\subsubsection{Finite Elements Implementation of Nonlinear Resilient Modulus}

The general characteristics of MEPDG in Finite Element Approach (FEA) for unbound materials and soils follow the two-dimensional asymmetric conditions. The displacement function over the element is given by (5).

$$
u=N q
$$

Where $\boldsymbol{u}^{T}=[u v]^{T}$ is the vector of displacement at a point in the $\mathrm{x}$ and $\mathrm{y}$ directions respectively. $\mathbf{N}$ is the matrix of interpolation functions, $q^{T}=\left[u_{1} v_{1} u_{2} \ldots u_{n} v_{n}\right]$ is the vector of nodal displacements, and $n$ is the number of nodes per element.

Equation 2.6 shows the incremental strain-displacement relationship. Where $\mathbf{B}$ is the strain-displacement transformation matrix, and $\boldsymbol{\varepsilon}$ is the vector of strains.

$$
d \boldsymbol{\varepsilon}=\boldsymbol{B} d \boldsymbol{q}
$$

The incremental stress-strain relationship is given by equation 2.7 . Where $\mathrm{C}$ is the constitutive matrix, and $\sigma$ is the stress vector. The components of $\mathrm{C}$ are function of tangent modulus, E, and the Poisson's ratio, $v$. in both linear and nonlinear behavior (5).

$$
d \boldsymbol{\sigma}=\boldsymbol{C} d \boldsymbol{\varepsilon}
$$

The minimum potential energy principle is used to define the element equilibrium which is given by equation 2.8. Where, $\Delta \mathrm{Q}$ is the vector of applied incremental nodal loads, $\Delta \mathrm{Q}_{\mathrm{r}}$ is the initial or unbalance load during nonlinear analysis, and the $\mathrm{k}$ is the element stiffness matrix as given by equation 2.9. Where, $\mathrm{V}$ is the volume of the element (5).

$$
\begin{aligned}
& \boldsymbol{k} \Delta \boldsymbol{q}=\Delta \boldsymbol{Q}+\Delta \boldsymbol{Q}_{r} \\
& \boldsymbol{k}=\int_{V} \boldsymbol{B}^{T} \boldsymbol{C} \boldsymbol{B} d_{V}
\end{aligned}
$$

An incremental iterative method is used to update the constitutive matrix, C, during each load increment in nonlinear analysis. The element stiffness matrices are assembled for all elements, the boundary conditions are introduced, and the resulting equation is solved for incremental displacements, stresses, and strains. These are 
accumulated over the load increments to compute the total displacements, stresses, and strains as function of load level (5).

Following the notation used in the MEPDG, the finite element solution at step $t+\Delta t$ requires that applied load to be in equilibrium with internal element stresses at step $t+\Delta \mathrm{t}$, so the summation of applied loads vector, $\mathbf{R}$, and internal element stresses vector, $\mathbf{F}$, to be zero (5).

$$
{ }^{\mathrm{t}+\Delta \mathrm{t}} \mathbf{R}-{ }^{\mathrm{t}+\Delta \mathrm{t}} \mathbf{F}=0
$$

During the iterative process the solution is known at step t so.

$$
{ }^{\mathrm{t}+\Delta \mathrm{t}} \mathbf{F}={ }^{\mathrm{t}} \mathbf{F}+\mathbf{F}
$$

Where, $\mathbf{F}$ is the incremental nodal forces corresponding to the incremental element stresses from step t to $t+\Delta \mathrm{t}$ and can be approximated at step $\mathrm{t}$ with the stiffness matrix, $\mathbf{K}$, and the vector of incremental nodal displacements, $\mathbf{U}$.

$$
\mathbf{F}={ }^{\mathrm{t}} \mathbf{K} \mathbf{U}
$$

The stiffness matrix is given by equation 2.13 (5). Where, the superscript $\mathrm{m}$ designates element $\mathrm{m}, V^{(m)}$ is the volume of the element $\mathrm{m}, \boldsymbol{B}^{(m)}$ is the strain-displacement interpolation matrix, $\boldsymbol{B}^{(m) T}$ is the transpose of $\boldsymbol{B}^{(m)},{ }^{t} \boldsymbol{C}^{(m)}$ is the constitutive matrix for element $\mathrm{m}$ at step $\mathrm{t}$, as in equation 14. e.g. for plane strain conditions, where ${ }^{t} \mathrm{E}_{\mathrm{T}}{ }^{(\mathrm{m})},{ }^{\mathrm{t}} v_{\mathrm{T}}{ }^{(\mathrm{m})}$ are the tangent elastic properties at step $\mathrm{t}$ for element $\mathrm{m}$.

$$
\begin{gathered}
{ }^{\mathrm{t}} \mathbf{K}=\sum_{\boldsymbol{m}} \int_{\boldsymbol{V}^{\boldsymbol{m}}} \boldsymbol{B}^{(m) T}\left({ }^{t} \boldsymbol{C}^{(m)}\right) \boldsymbol{B}^{(m)} d_{V} \\
{ }^{\mathrm{t}} \mathbf{C}^{(\mathrm{m})}=\frac{{ }^{t} E_{T}^{(m)}\left(1-{ }^{t} v_{T}^{(m)}\right)}{\left(1+{ }^{t} \boldsymbol{v}_{T}^{(m)}\right)\left(1-2^{t} v_{T}^{(m)}\right)}\left[\begin{array}{ccc}
1 & \frac{{ }^{t} v_{T}^{(m)}}{\left(1-{ }^{t} v_{T}^{(m)}\right)} & 0 \\
\frac{{ }^{t} v_{T}^{(m)}}{\left(1-{ }^{t} v_{T}^{(m)}\right)} & 1 & 0 \\
0 & 0 & \frac{\left(1-2^{t} v_{T}^{(m)}\right)}{2\left(1-{ }^{t} v_{T}^{(m)}\right)}
\end{array}\right]
\end{gathered}
$$

The displacement, $\mathbf{U}$, can be computed by equation 2.15 which results from the combination of equations 2.10,2.11, and 2.12. The approximation of displacement at step $\mathrm{t}+\Delta \mathrm{t}$ is given for equation $2.16(5)$. 


$$
\begin{aligned}
& { }^{\mathrm{t}} \mathbf{K} \mathbf{U}={ }^{\mathrm{t}+\Delta \mathrm{t}} \mathbf{R}-{ }^{\mathrm{t}} \mathbf{F} \\
& { }^{\mathrm{t}+\Delta \mathrm{t}} \mathbf{U} \approx{ }^{\mathrm{t}} \mathbf{U}+\mathbf{U}
\end{aligned}
$$

Newton-Raphson iterations procedure is used to reduce the error of the approximate solution defined by equations 2.15, and 2.16 (5). The initial condition are ${ }^{\mathrm{t}+\Delta t} \mathbf{U}^{(0)}={ }^{\mathrm{t}} \mathbf{U},{ }^{\mathrm{t}+\Delta t} \mathbf{K}^{(0)}={ }^{\mathrm{t}} \mathrm{K}$ and the iterations are given for equations 2.17 and 2.18 (5). Where, i identifies the iteration cycles, ${ }^{\mathrm{t}+\Delta t} \mathbf{R}-{ }^{\mathrm{t}+\Delta t} \mathbf{F}^{(\mathrm{i}-1)}$ represents the unbalance vector when the loads are not balanced with the element stresses, and $\Delta \mathbf{U}^{(\mathrm{i})}$ represents the increment in nodal displacements to bring the element to equilibrium.

$$
\begin{aligned}
& { }^{\mathrm{t}+\Delta \mathrm{t}} \mathbf{K}^{(\mathrm{i}-1)} \Delta \mathbf{U}^{(\mathrm{i})}={ }^{\mathrm{t}+\Delta \mathrm{t}} \mathbf{R}-{ }^{\mathrm{t}+\Delta \mathrm{t}} \mathbf{F}^{(\mathrm{i}-1)} \\
& { }^{\mathrm{t}+\Delta \mathrm{t}} \mathbf{U}^{(\mathrm{i})}={ }^{\mathrm{t}+\Delta \mathrm{t}} \mathbf{U}^{(\mathrm{i}-1)}+\Delta \mathbf{U}^{(\mathrm{i})}
\end{aligned}
$$

The strain and stresses for the elements are also computed during the iteration process according equation 2.19, 2.20, 2.21 (5). Where the initial conditions ${ }^{\mathrm{t}+\Delta \mathrm{t}} \boldsymbol{\varepsilon}^{(\mathrm{m})(0)}=$

$$
\begin{aligned}
{ }^{\mathrm{t}} \boldsymbol{\varepsilon}^{(\mathrm{m})},{ }^{\mathrm{t}+\Delta \mathrm{t}} \boldsymbol{\sigma}^{(\mathrm{m})(0)}={ }^{\mathrm{t}} \boldsymbol{\sigma}^{(\mathrm{m})}, \text { and }{ }^{\mathrm{t}+\Delta \mathrm{t}} \boldsymbol{C}^{(\mathrm{m})(0)}={ }^{\mathrm{t}} \mathbf{C}^{(\mathrm{m})} . \\
\Delta \boldsymbol{\varepsilon}^{(\mathrm{m})(\mathrm{i})}=\mathbf{B}^{(\mathrm{m})} \Delta \mathbf{U}^{(\mathrm{i})} \\
{ }^{\mathrm{t}+\Delta \mathrm{t}} \boldsymbol{\varepsilon}^{(\mathrm{m})(\mathrm{i})}={ }^{\mathrm{t}+\Delta \mathrm{t}} \boldsymbol{\varepsilon}^{(\mathrm{m})(\mathrm{i}-1)}+\Delta \boldsymbol{\varepsilon}^{(\mathrm{m})(\mathrm{i})} \\
{ }^{\mathrm{t}+\Delta \mathrm{t}} \boldsymbol{\sigma}^{(\mathrm{m})(\mathrm{i})}={ }^{\mathrm{t}+\Delta \mathrm{t}} \boldsymbol{\sigma}^{(\mathrm{m})(\mathrm{i}-1)}+{ }^{\mathrm{t}+\Delta \mathrm{t}} \boldsymbol{C}^{(\mathrm{m})(\mathrm{i}-1)} \Delta \boldsymbol{\varepsilon}^{(\mathrm{m})(\mathrm{i})}
\end{aligned}
$$

\subsection{Response Models of Flexible Pavements}

The response of the pavement under traffic loads and environmental conditions is determined by the pavement response models. The inputs to the models are pavement characteristics such as layer thickness, environmental condition such as temperature and moisture, material properties such as elastic and nonlinear properties. The outputs of this model are the stresses, strains and displacements within the pavement layers at critical locations. These responses are used to accumulate damage over time in the MEPDG damage models. The objective of MEPDG is to design pavements based on predicted pavement performance, therefore the critical pavement response quantities are the primary output of the response model (1). 
The response model included in the MEPDG for flexible pavements is the multilayer elastic theory (MLET) program JULEA for linear elastic analysis. The 2-D finite element program DSC2D is included for use in Level 1 for non-liner moduli analysis of unbound layer materials such as bases, subbases or subgrades. The DSC2D model was not included in the calibration effort and it is suggested for research only (1).

The JULEA program provides a combination of analyses features, theoretical rigor, and computational speed for linear analyses. It also requires minimal input data from the user; the only inputs required are the layer thicknesses, elastic modulus and Poisson's ratio for each layer, the tire pressure, tire spacing, and the tire contact area. Solution for multiple wheel loads can be computed automatically by the program using the asymmetric single wheel solution via superposition (5).

DSC2D is a finite element analysis program for computation stress, strain, and displacements in nolinear pavement system. The program provides capability to perform analyses for asymmetric nonlinear formulation, stress dependant resilient modulus for unbound pavement layers, different interface conditions between layers (full-slip, no-slip, and intermediate), and infinite boundary elements for reducing total analysis model size. DSC2D was modified from its original version developed at University of Arizona by C.S. Desai (5).

\subsection{Pavement Performance}

The pavement performance concept is related to the condition of the pavement over time. Changes in pavement condition define pavement performance. The general view or pavement performance includes functional performance, structural performance, and safety. The MEPDG includes structural and functional performance models. The structural performance of a flexible pavement is associated with its physical condition, mainly fatigue cracking and rutting. These distresses are predicted by MechanisticEmpirical concepts and are incorporated in the design process. Functional performance refers to how well the pavement serves to user. The major characteristic of functional performance is the ride quality that is quantified by the serviceability-performance 
concept developed at the AASHO Road Test (1). Ride quality is evaluated in term of International Roughness Index, IRI, in MEPDG.

\subsubsection{Pavement Performance Models}

The functional performance is related to the ride quality or pavement roughness as an expression of irregularities in the pavement surface that adversely affect the ride quality of vehicles. The 1993 AASHTO design procedure used the serviceability concept and the present serviceability index (PSI) was obtained from measurements of roughness and distress (1). The parameter used in the MEPDG is the roughness which is measured by the international roughness index (IRI). Distresses such as cracking, rutting, punchouts, and faulting are the major factors affecting the roughness; the quality of materials, the environmental conditions and the traffic load are related to the development of roughness. Figure 2.5 illustrates the pavement performance trends in terms of IRI and serviceability (1).

Increments in distress cause increments in roughness thus decrease in ride quality. The general structure for the roughness models is as follows;

$$
S_{(t)}=S_{0}+\left(a_{1} S_{D_{(t)} 1}+a_{2} S_{D_{(t)}}+\cdots+a_{n} S_{D_{(t)}}\right)+b_{j} S_{j}+c_{j} M_{j}
$$

Where;

$-S_{(t)}=$ pavement roughness at a specific time, $t(I R I, i n / m i)$.

- $\mathrm{S}_{0}=$ initial roughness immediately after construction (IRI, in/mi).

- $\mathrm{S}_{\mathrm{D}_{(\mathrm{t})} \mathrm{i}}(\mathrm{i}=1$ to $\mathrm{n})=$ change of roughness due to its distress at a given time $\mathrm{t}$ in the analysis period.

- $\quad a_{i}(i=1 \ldots n), b_{j}, c_{j}=$ regression constants.

- $\quad \mathrm{S}_{\mathrm{j}}=$ change in roughness due to site factors (subgrade and age)

- $\quad \mathrm{M}_{\mathrm{j}}=$ change in roughness due to maintenance activities. 


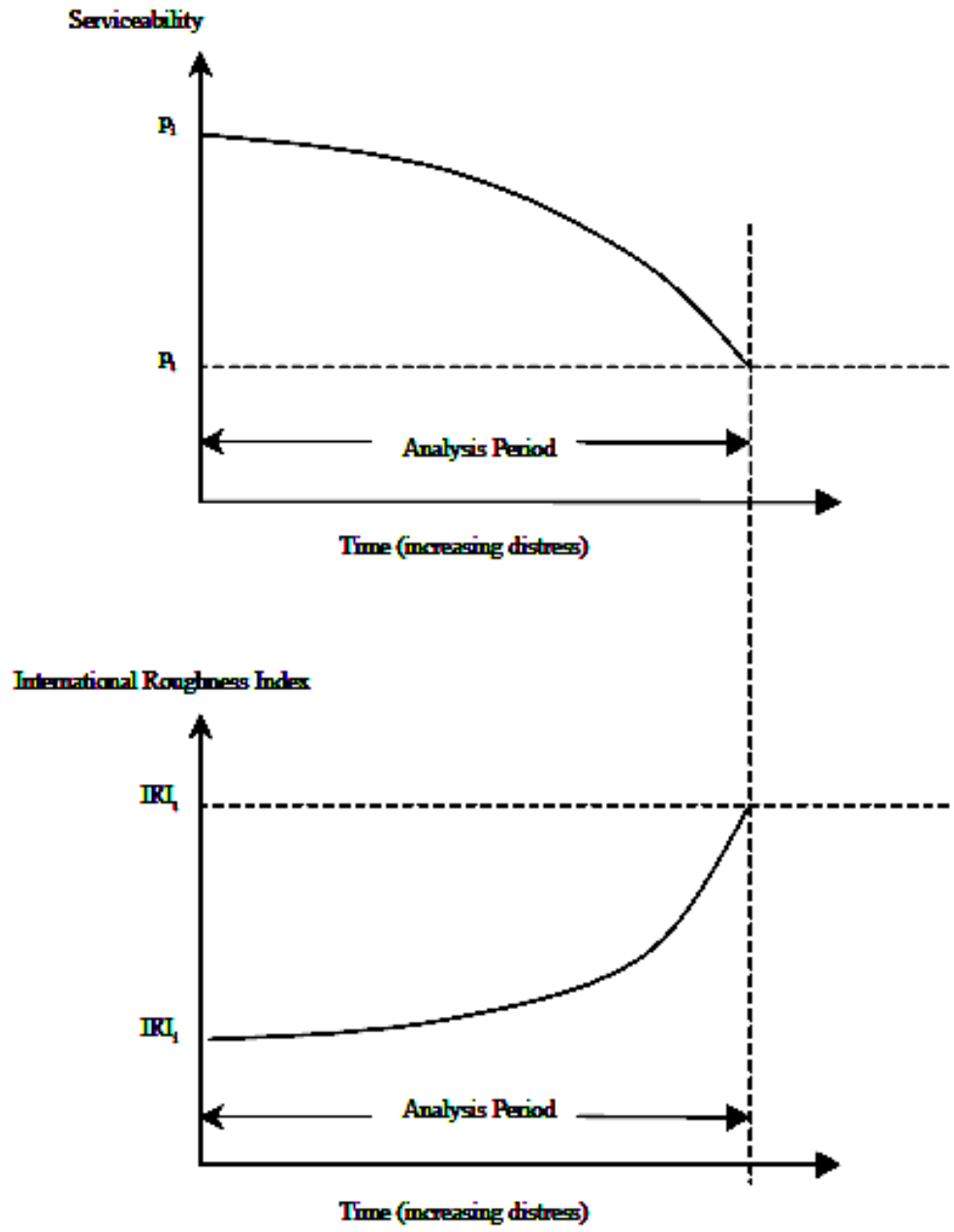

FIGURE 2.5 Pavement Performance Trends (NCHRP, 2004)

\subsubsection{Distress Prediction Equations for Flexible Pavement}

The MEPDG includes transfer functions and regression equations to estimate the performance of the pavements. The performance indicators were calibrated using data from the Long-Term Pavement Performance (LTPP) database. The indicators used in the MEPDG for flexible pavements are listed as follows (2):

- Smoothness (IRI)

- Total rut depth in HMA, unbound aggregate base, and subgrade.

- Non-load-related transverse cracking 
- Load-related fatigue cracking, bottom initiated cracks

- Load-related longitudinal cracking, surface initiated cracks

- Reflection cracking in HMA overlays and joint in exiting flexible pavements, semi-rigid, composite, and rigid pavements

\subsubsection{Smoothness}

The MEPDG basis for predicting smoothness degradation is that the increase of roughness is due to surface distress, site factor, and maintenance. It is reflected in an increase of the value of IRI. The following equations are used to predict IRI over time in Hot-Mix Asphalt (HMA) pavements (2).

\section{- New HMA Pavements and Overlays of Flexible Pavements}

$$
I R I=I R I_{0}+0.0150(S F)+0.40\left(F C_{\text {Total }}\right)+0.0080(T C)+40.0(R D)
$$

Where,

- $\quad I R I_{0}=$ Initial IRI after construction, $\mathrm{in} / \mathrm{mi}$

$-\mathrm{SF}=$ Site factor (equation 2.24)

- $\quad \mathrm{FC}_{\text {Total }}=$ Area of fatigue as percent of total lane area. (Combined alligator, longitudinal, and reflection cracking in the wheel path. $1 \mathrm{ft}$ to convert length into area basis.).

- $\quad$ TC $=$ Length of transverse cracking (including the reflection of transverse cracks in existing HMA pavements), $\mathrm{ft} / \mathrm{mi}$.

- $\quad \mathrm{RD}=$ Average rut depth (in)

$$
S F=\operatorname{Age}[0.02003(P I+1)+0.007947(P \operatorname{ercip}+1)+0.000636(F I+1)]
$$

Where,

- Age = Pavement age, yr

- $\quad$ PI $=$ Percent plasticity index of the soil,

- Precip = Average annual precipitation or rainfall, in

- $\mathrm{FI}=$ Average annual freezing index, ${ }^{\circ} \mathrm{F}$-days

To compute the monthly or annual freezing index, the following equation is used: 


$$
F I=\sum_{i=1}^{n}\left(0-T_{i}\right)
$$

where:

- $\quad \mathrm{FI}=$ freezing index, degrees Celsius $\left({ }^{\circ} \mathrm{C}\right)$ degree-days

- $\mathrm{Ti}=$ average daily air temperature on day $\mathrm{i},{ }^{\circ} \mathrm{C}$

- $\mathrm{n}=$ days in the specified period when average daily temperature is below freezing

- $\quad \mathrm{i}=$ number of days below freezing

Only the days where the average daily temperature is below freezing are used. Therefore, the freezing index is the negative of the sum of all average daily temperatures below $0^{\circ} \mathrm{C}$ within the given period.

\section{- HMA Overlays of Rigid Pavements}

$I R I=I R I_{0}+0.00825(S F)+0.575\left(F C_{\text {Total }}\right)+0.0014(T C)+40.8(R D)$

The terms are defined as above.

\subsubsection{Rut Depth}

Rutting is the plastic and permanent vertical deformation in a pavement structure and includes the permanent deformation in the HMA, unbound layers, and the subgrade. The MEPDG rutting calculation is based on the incremental distortion within each layer (2). The constitutive relationship for flexible pavement used in the MEPDG is based upon the laboratory repeated load permanent deformation test which was statistical field calibrated (1). MEPDG subdivides the pavement structure into sublayers for modeling the temperature and moisture variations. Rutting is computed for each sublayer and the overall permanent deformation is obtained with the following (2). The accumulated permanent deformation is given by (1).

$$
\Delta_{p}=\sum_{\mathrm{i}=1}^{\text {nsublayers }} \varepsilon_{p}^{i} h^{i}
$$

Where,

- $\Delta_{p}=$ Accumulated permanent or plastic vertical deformation in layer or sublayer, in. 
- $\quad$ nsublayers $=$ Number of sublayers

- $\varepsilon_{p}=$ Plastic axial strain in layer or sublayer, in/in.

- $\quad h=$ Thickness of layer or sublayer, in.

\section{- Rut Depth Model for HMA (2)}

$$
\varepsilon_{p(H M A)}=\beta_{1} k_{z} \varepsilon_{r(H M A)} 10^{k_{r 1}} n^{k_{1 r} \beta_{2 r}} T^{k_{3 r} \beta_{3 r}}
$$

Where,

- $\varepsilon_{p(H M A)}=$ Accumulated permanent or plastic axial strain in the HMA layer or sublayer ( in/in).

- $\varepsilon_{r(H M A)}=$ Resilient or elastic strain from structural response model at the middepth of each HMA sublayer (in/in).

- $\quad n=$ Number of axle-load repetitions. (For analysis period and axle types)

- $\mathrm{T}=$ Mix or pavement temperature, ${ }^{\circ} \mathrm{F}$.

- $\quad \mathrm{k}_{\mathrm{z}}=$ Depth confinement factor (Equation 2.28).

- $\mathrm{k}_{1 \mathrm{r},} \mathrm{k}_{2 \mathrm{r}}, \mathrm{k}_{3 \mathrm{r}}=$ Global field calibration parameter $\left(\mathrm{k}_{1 \mathrm{r}}=-3.35412, \mathrm{k}_{2 \mathrm{r}}=0.4791, \mathrm{k}_{3 \mathrm{r}}=\right.$ 1.5606)

- $\beta_{1 r}, \beta_{2 r}, \beta_{3 r}=$ Local or mixture field calibration constants (Set to 1.0 for the national calibration).

$$
\begin{aligned}
& k_{z}=\left(C_{1}+C_{2} D\right) 0.328196^{D} \\
& C_{1}=-0.1039\left(H_{H M A}\right)^{2}+2.4868 H_{H M A}-17.342 \\
& C_{2}=0.0172\left(H_{H M A}\right)^{2}-1.733 H_{H M A}+27.428
\end{aligned}
$$

Where,

- $\quad \mathrm{D}=$ Depth below the surface, in.

- $H_{H M A}=$ Total HMA thickness, in. 


\section{- Rut Depth Model for Unbound Layers, Foundations, and Embankments (2)}

Equation 2.31 is used for granular and soil materials.

$$
\Delta_{p(\text { soil })}=\beta_{s 1} k_{s 1} \varepsilon_{v} h_{\text {soil }}\left(\frac{\varepsilon_{0}}{\varepsilon_{r}}\right) e^{-\left(\frac{\rho}{n}\right)^{\beta}}
$$

Where,

- $\Delta_{p(\text { soil })}=$ Permanent or plastic deformation for the granular layer or sublayer, in

- $\quad n=$ number of axle-load application.

- $\varepsilon_{0}=$ Intercept determined from laboratory repeated load permanent deformation test, in/in.

- $\varepsilon_{r}=$ Resilient strain imposed in laboratory test to obtain material properties, $\varepsilon_{0}, \varepsilon$, and $\rho$, in/in.

- $\varepsilon_{v}=$ Average vertical resilient or elastic strain in the granular layer or sublayer and calculated by structural response model.

- $\quad h_{\text {soil }}=$ Thickness of the unbound layer or sublayer, in.

- $\quad k_{s 1}=$ Global calibration coefficients; $k_{s 1}=1.673$ for granular materials and 1.35 for fine-grained material.

- $\quad \beta_{s 1}=$ Local calibration constant for the rutting in the unbound layers; It was set to 1.0 for the global calibration effort.

$$
\begin{aligned}
& \log \beta=-0.61119-0.017638\left(w_{c}\right) \\
& \rho=10^{9}\left(\frac{C_{0}}{\left(1-\left(10^{9}\right)^{\beta}\right)}\right)^{\frac{1}{\beta}} \\
& C_{0}=\ln \left(\frac{a_{1} M_{r}^{b_{1}}}{a_{9} M_{r}^{b_{9}}}\right)=0.0075
\end{aligned}
$$

Where,

$-w_{c}=$ water content, $\%$

- $\quad M_{r}=$ Resilient modulus of the unbound layer or sublayer, psi.

- $\mathrm{a}_{1,9}=$ Regression constants; $\mathrm{a}_{1}=0.15$ and $\mathrm{a}_{9}=20$

- $\quad b_{1,9}=$ Regression constants; $b_{1}=0.0$ and $b_{9}=0.0$ 


\subsubsection{Load Related Cracking}

The MEPDG has models for prediction of alligator cracking and longitudinal cracking. Longitudinal cracking is assumed to develop from top to bottom while alligator cracking is assumed to develop from bottom to top. Equation 2.35 is used to compute the allowable number of axle-load applications to predict fatigue cracking with an incremental damage index approach from equation 2.40 (2).

$$
N_{f-H M A}=k_{f 1} \beta_{f 1}(C)\left(C_{H}\right)\left(\varepsilon_{t}\right)^{k_{f 2} \beta_{f 2}}\left(E_{H M A}\right)^{k_{f 3} \beta_{f 3}}
$$

Where,

$-\quad N_{f-H M A}=$ Allowable number of axle-load applications.

- $\varepsilon_{t}=$ Tensile strain at critical locations from structural response model, in./in.

- $\quad E_{H M A}=$ Dynamic modulus of the asphalt mix measured in compression, psi.

- $\quad k_{f 1}, k_{f 2}, k_{f 3}=$ Global field calibration parameters $\left(k_{f 1}=0.007566, k_{f 2}=-3.9492\right.$, and $\left.k_{f 3}=-1.281\right)$.

- $\beta_{f 1}, \beta_{f 2}, \beta_{f 3=}$ Local field calibration constants (these constants were set to 1.0 in the global calibration)

$$
C=10^{M}
$$

$$
M=4.84\left(\frac{V_{b e}}{V_{a}+V_{b e}}-0.69\right)
$$

Where,

- $\quad V_{b e}=$ Percent of effective asphalt content by volume.

- $V_{a}=$ Percent of air voids in the asphalt mix.

- $C_{H=}$ Factor for thickness correction that depends on type of cracking from the following equations.

- For Bottom-up or Alligator Cracking:

$$
\mathrm{C}_{\mathrm{H}}=\frac{1}{0.000398+\frac{0.003602}{1+\mathrm{e}^{\left(11.02-3.49 \mathrm{H}_{\mathrm{HMA}}\right)}}}
$$




\section{- For Top-down or Longitudinal Cracking:}

$$
\mathrm{C}_{\mathrm{H}}=\frac{1}{0.01+\frac{12}{1+\mathrm{e}^{\left(15.676-2.8186 \mathrm{H}_{\mathrm{HMA}}\right)}}}
$$

Where,

- $H_{H M A}=$ Total HMA thickness, in.

The incremental damage index is computed as the ratio of actual number of axleload to the allowable number of axle-load in the corresponding time period and axle-load intervals for each axle type. The cumulative damage index (DI) is the summation of the incremental damage index over the time as seen in equation 2.40 (2).

$$
D I=\sum(\Delta D I)_{j, m, l, p, T}=\sum\left(\frac{n}{N_{f-H M A}}\right)_{j, m, l, p, T}
$$

Where,

- $n=$ number of axle-load applications in the time period,

- $\mathrm{j}=$ Axle-load interval,

- $\mathrm{m}=$ Axle-load type,

- $1=$ Truck type according to the truck classification groups in MEPDG,

- $\mathrm{p}=$ Month, and

- $\quad \mathrm{T}=$ Median temperature for five temperature subdivisions of each month, ${ }^{\circ} \mathrm{F}$

The following transfer functions are used to compute length of longitudinal cracking and area alligator cracking from equation 2.40. To predict alligator cracking on area basis, equation 2.41 is used.

$$
F C_{\text {bottom }}=\left(\frac{1}{60}\right)\left(\frac{C_{4}}{1+e^{\left(C_{1} C_{1}{ }^{*}+C_{2} C_{2}{ }^{*} \log \left(D I_{\text {bottom }} * 100\right)\right)}}\right)
$$

Where:

- $F C_{\text {bottom }}=$ Area of alligator cracking ( $\%$ of the total lane area).

- $D I_{\text {bottom }}=$ Cumulative damage index at the bottom of the asphalt layers.

- $C_{1,2,4}=$ regression constants $\left(C_{4}=6,000 ; C_{1}=1.0 ;\right.$ and $\left.C_{2}=1.0\right)$. 


$$
\begin{aligned}
& C_{1}{ }^{*}=-2 C_{2}{ }^{*} \\
& C_{2}{ }^{*}=-2.40874-39.748\left(1+H_{H M A}\right)^{-2.856}
\end{aligned}
$$

Where,

- $H_{H M A}=$ Total HMA thickness, in.

The length of longitudinal fatigue cracking is predicted by equation 2.43 .

$$
F C_{T o p}=10.56\left(\frac{C_{4}}{\left.1+e^{\left(C_{1}-C_{2} \log \left(D I_{T o p}\right)\right.}\right)}\right)
$$

Where:

- $F C_{\text {Top }}=$ Length of longitudinal cracks $(\mathrm{ft} / \mathrm{mi})$

- $D I_{\text {Top }}=$ Cumulative damage index near the top of the HMA surface.

- $\quad C_{1,2,4}=$ Regression constants $\left(C_{1}=7.0 ; C_{2}=3.5\right.$; and $\left.C_{4}=1,000\right)$.

\subsubsection{Fatigue Cracking for Cement Treated Bases}

Cracking in cement treated bases are computed from the following transfer functions (2).

$$
\begin{aligned}
& N_{f-C T B}=10\left[\frac{k_{C 1} \beta_{c 1}\left(\frac{\sigma_{t}}{M_{R}}\right)}{k_{c 2} \beta_{c 2}}\right] \\
& F C_{C T B}=C_{1}+\frac{C_{2}}{1+e^{\left(C_{3}-C_{4} \log \left(D I_{C T B}\right)\right)}}
\end{aligned}
$$

Where,

- $\quad N_{f-С T B}=$ Allowable number of axle-load applications.

- $\sigma_{t}=$ Tensile stress at the bottom of the CTB layer, psi.

- $\quad M_{R}=28$-day modulus of rupture for the CTB layer, psi

- $D I_{C T B}=$ Cumulative damage index of the CTB layer computed from with Equation 2.40,

- $\quad k_{c 1, c 2}=$ Global calibration factors (set to 1.0 in global calibration and $k_{c 1}$ $=0.972$ and $k_{c 2}=0.0825$ from other studies)

- $\quad \beta_{c 1, c 2}=$ Local calibration constants (set to 1.0 in the global calibration).

$-F C_{C T B}=$ Area of fatigue cracking $(\mathrm{sq} \mathrm{ft})$. 


$$
\text { - } C_{1,2,3,4}=\text { Regression constants }\left(C_{1}=1.0, C_{2}=1.0, C_{3}=0 \text {, and } C_{4}=1,000\right)
$$

For computing the critical pavement response in the CBT or other pavement layers, equation 2.46 is used. The damage modulus approach is used for incremental fatigue cracking in a semi-rigid pavement.

$$
E_{C T B}^{D(t)}=\mathrm{E}_{\mathrm{CTB}}^{\mathrm{Min}}+\left(\frac{\mathrm{E}_{\mathrm{CTB}}^{\mathrm{Max}-\mathrm{E}_{\mathrm{CTB}}^{\mathrm{Min}}}}{1+\mathrm{e}^{\left(-4+4.14\left(\mathrm{DI}_{\mathrm{CTB}}\right)\right)}}\right)
$$

Where,

- $\quad E_{C T B}^{D_{(t)}}=$ Equivalent damaged elastic modulus at time $\mathrm{t}$ for the CTB layer, psi,

- $\quad E_{C T B}^{M i n}=$ Equivalent elastic modulus for total destruction of the CTB layer, psi, and

- $\quad E_{C T B}^{M a x}=28$-day elastic modulus of the intact CTB layer, no damage, psi.

\subsubsection{Non-load Related Cracking}

The model for thermal cracking is a modified version of the model developed for the Strategic Highway Research Program (SHRP) and the Paris law of crack propagation. The MEPDG model for computing the amount of crack propagation induced by thermal cooling cycles is: (2).

$$
\Delta C=A(\Delta K)^{n}
$$

Where,

- $\Delta C=$ Change in the crack depth induced by a cooling cycle.

- $\Delta K=$ Change in the stress intensity factor induced by cooling cycle.

- $\mathrm{A}, \mathrm{n}=$ Fracture parameters for the HMA mixture.

A and $\mathrm{n}$ can be computed from equations 2.48 and 2.49 respectively (2).

$$
\begin{aligned}
& A=10^{K_{t} \beta_{t}\left(4.839-2.52 \log \left(E_{H M A} \sigma_{m} n\right)\right)} \\
& n=0.8\left[1+\frac{1}{m}\right]
\end{aligned}
$$

Where, 
- $K_{t}=$ Global calibration coefficient which depended on the input level (Level 1=5.0; Level 2=1.5; and Level 3=3.0).

- $\quad E_{H M A}=$ HMA indirect tensile modulus (psi).

- $\sigma_{m}=$ Mixture tensile strength (psi).

- $m=$ The $m$-value from the indirect tensile creep compliance laboratory curve.

- $\beta_{t}=$ Local calibration factor.

The stress intensity factor, $\mathrm{K}$, was incorporated in the MEPDG as:

$$
K=\sigma_{\text {tip }}\left[0.45+1.99\left(\mathrm{C}_{0}\right)^{0.56}\right]
$$

Where:

- $\sigma_{\text {tip }}=$ Stress in the asphalt concrete at depth of crack tip (psi) from pavement response model.

- $C_{0}=$ Current crack length, ft.

The amount of cracking is computed with the following equation (2):

$$
T C=\beta_{t 1} N\left[\frac{1}{\sigma_{d}} \log \left(\frac{c_{d}}{H_{H M A}}\right)\right]
$$

Where,

- $\mathrm{TC}=$ Estimated amount of thermal cracking, $\mathrm{ft} / \mathrm{mi}$,

- $\quad \beta_{t 1}=$ Global calibration regression coefficient (400).

$-c_{d}=$ Crack depth, in.

- $\quad H_{H M A}=$ Thickness of asphalt layers, in.

- $\quad N\left[\frac{1}{\sigma_{d}} \log \left(\frac{c_{d}}{H_{H M A}}\right)\right]=$ The probability that $\log \left(\mathrm{C}_{\mathrm{d}}\right)>\log \left(\mathrm{H}_{\mathrm{HMA}}\right)$ in standard normal distribution. This probability is determined by assuming that the logarithm of the depth of cracks in the pavement is normally distributed with mean equal to $\log \mathrm{C}_{\mathrm{o}}$ (crack depth predicted by the model) and a variance of $\sigma^{2}(6)$.

- $\sigma_{d}=$ Standard deviation of the log of the depth of cracks in the pavement $(0.769$, in., from the model national calibration). 


\subsubsection{Reflection Cracking in HMA Overlay}

The area of reflection crack is calculated as a function of time using a sigmoid function. However, this empirical equation was not globally calibrated (2). The MEPDG includes user-defined cracking progression parameters $\mathrm{c}$, and $\mathrm{d}$ to accelerate or delay the amount of reflection crack (Table 2.1). They need to be calibrated locally. Meanwhile, they can be considered with caution (2).

$$
R C=\frac{100}{1+e^{a(c)+b t(d)}}
$$

Where,

- $\quad \mathrm{RC}=$ Percent of cracks reflected. (A width of crack of $1 \mathrm{ft}$ is considered to compute the percent area).

$-\mathrm{t}=$ Time, $\mathrm{yr}$,

- $\quad \mathrm{a}, \mathrm{b}=$ Calibration regression coefficients.

- $\quad \mathrm{c}, \mathrm{d}=$ User-defined cracking progression parameters .

$$
\begin{aligned}
& a=3.5+0.75\left(H_{e f f}\right) \\
& b=-0.688684-3.37302\left(H_{e f f}\right)^{-0.915469}
\end{aligned}
$$

Where,

\begin{tabular}{|c|c|c|c|c|}
\hline \multirow[b]{3}{*}{ Pavement Type } & \multicolumn{4}{|c|}{ Fitting and user defined parameters } \\
\hline & \multirow{2}{*}{$\begin{array}{l}\mathbf{a}, \mathbf{b} \\
\mathbf{H}_{\text {eff }}\end{array}$} & \multirow[t]{2}{*}{$\mathbf{c}$} & \multicolumn{2}{|c|}{ d } \\
\hline & & & $\begin{array}{c}\text { Delay cracking } \\
\text { by } 2 \mathrm{yr}\end{array}$ & $\begin{array}{c}\text { Acceletate cracking } \\
\text { by } 2 \mathrm{yr}\end{array}$ \\
\hline Flexible & $\mathrm{H}_{\mathrm{eff}}=\mathrm{H}_{\mathrm{HMA}}$ & - & - & - \\
\hline Rigid-good load transfer & $\mathrm{H}_{\mathrm{eff}}=\mathrm{H}_{\mathrm{HMA}}-1$ & - & - & - \\
\hline Rigid-poor load transfer & $\mathrm{H}_{\mathrm{eff}}$ & - & - & - \\
\hline \multicolumn{5}{|l|}{$\begin{array}{l}\text { Effective overlay } \\
\text { thickness, } H_{\text {eff }}, \text { in. }\end{array}$} \\
\hline$<4$ & - & 1.0 & 0.6 & 3.0 \\
\hline $4-6$ & - & 1.0 & 0.7 & 1.7 \\
\hline$>6$ & - & 1.0 & 0.8 & 1.4 \\
\hline
\end{tabular}

- $\quad H_{e f f}=$ Effective HMA overlay thickness according to Table 2.1 (2).

TABLE 2.1 Regression fitting parameters (AASHTO, 2008) 
The continuous damage accumulation of these layers is considered in the MEPDG. For a given month $(\mathrm{m})$ the total reflection damage is computed as:

$$
D I_{m}=\sum_{i=1}^{m} \Delta D I_{i}
$$

Where,

- $\quad D I_{m}=$ Damage index for month $\mathrm{m}$, and

- $\Delta D I_{i}=$ Increment of damage index in month $\mathrm{i}$.

The area of reflection damage for the underlying layer at month $\mathrm{m}$ is computed with the following equation:

$$
C A_{m}=\frac{100}{1+e^{6-(6 D I m)}}
$$

Equation 2.57 is used to compute the amount of cracking reflected from the stabilized layer to the surface for a month $\mathrm{m}$; for each month $\mathrm{i}$, the increment of damage is $\Delta$ DIi that produce an increment in the cracking area $\mathrm{CA}_{\mathrm{i}}$.

$$
T R A_{m}=\sum_{i=1}^{m} R C_{t}\left(\Delta C A_{i}\right)
$$

Where,

- $T R A_{m}=$ Total reflected cracking area for month $\mathrm{m}$,

- $R C_{t}=$ Percent cracking reflected for age $\mathrm{t}$ (in years) from Equation 2.55

- $\Delta C A_{i}=$ Increment of fatigue cracking for month i.

\subsection{Traffic}

Traffic loads are applied to the pavement throughout its design life. The MEPDG considers the traffic loads in terms of load distribution. Truck volumes and loads are characterized in terms of the full axle-load spectrum data for vehicle classes 4 and above as defined on Figure 2.6. The traffic data collection is consistent with actual practice of highway agencies, weight in motion (WIM), automatic vehicle classification (AVC), and vehicle counts are used (1). 


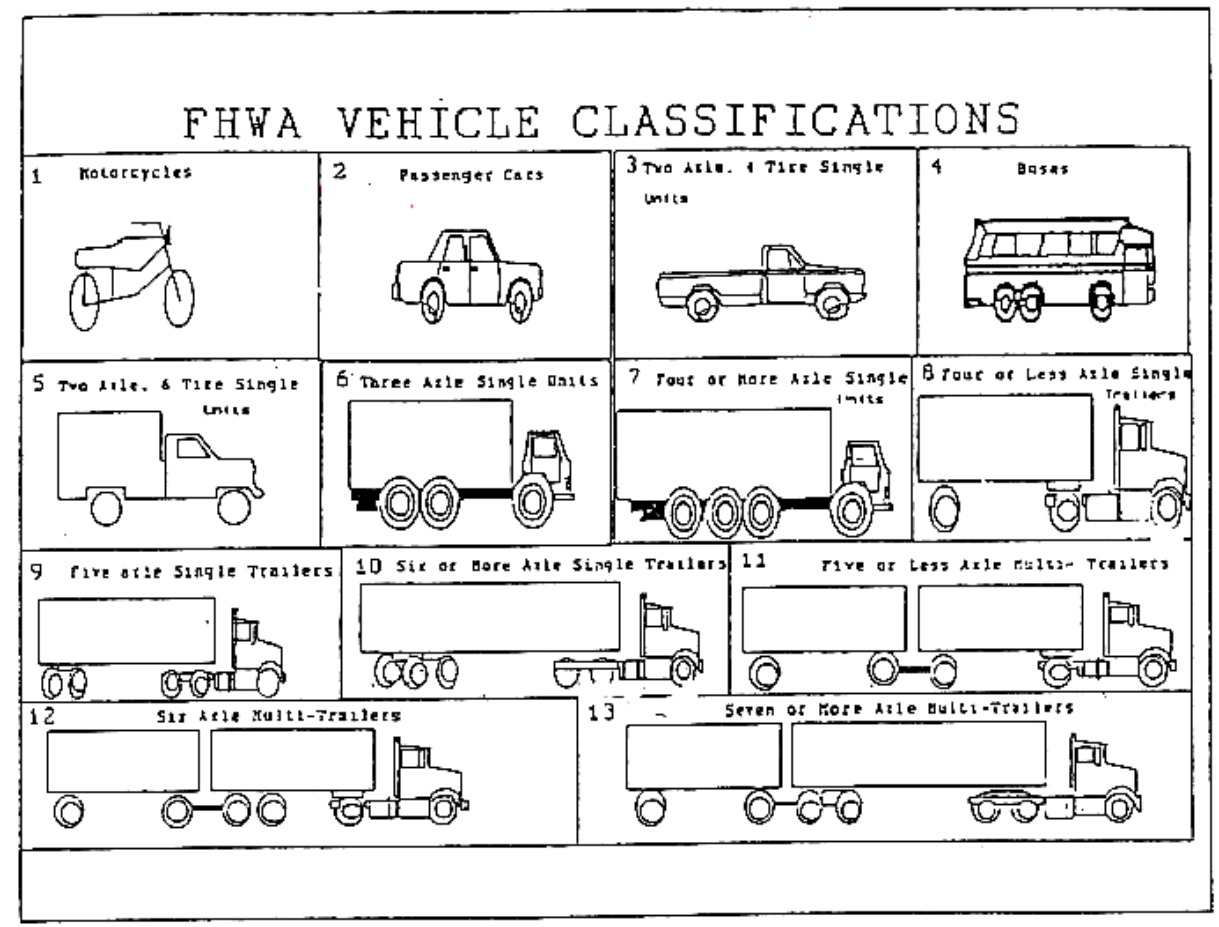

FIGURE 2.6 Vehicle classification (NCHRP, 2004)

Some agencies may not have the full axle-load spectrum as needed in the MEPDG. Additionally, the resources for data gathering may not be available in some agencies. The hierarchical approach is used for traffic data in order to make possible the implementation of the design method regardless of traffic information available. The MEPDG has three levels for traffic data input from one to three. The levels are defined according to the knowledge of the past and future traffic characteristics (1).

- Traffic Level 1: This level uses traffic spectrum directly gathered at the project site or near to it. It is the most accurate estimation of the traffic and uses actual axle weights and truck traffic volume distributions.

- Traffic Level 2: The data required for level 2 are the same as level 1 with the difference that for this level the truck weights are taken from regional weight summaries. The truck volumes have to be taken accurately by collection of enough information at project site or near to it. This information includes any weekday/weekend volume variation and any seasonal trends in truck loads. 
- Traffic Level 3: This level is used when there is not enough truck volume information. The loads can be taken from regional or statewide load distributions. Generally, the designer has the AADT and percent of trucks for the project site. The MEPDG provides some default load distributions that can be used for level 3.

\subsubsection{Traffic Input Parameters}

The structural design of pavements with MEPDG requires four types of traffic data (1).

- Traffic volume

- Traffic volume adjustment factors

- Monthly adjustment

- Vehicle class distribution

- Hourly truck distribution

- Traffic growth factors

- Axle load distribution factors

- General traffic inputs

- Number axles/trucks

- Axle configuration

- Wheel base

\subsubsection{Traffic Volume}

The first year that the pavement is under traffic is defined as base year. The input data for the base year are described as follows (1).

- Two-Way Annual Average Daily Track Traffic (AADTT)

Two-Way AADTT is the total number of truck traffic passing over a pavement structure in both directions during a period of 24 hours.

\section{- Number of Lanes in the Design Direction}

Number of lanes in the design direction is the total number of lanes with traffic in the same direction. It is defined according to design specifications. 


\section{- Percent of Trucks in the Design Direction}

The percent of trucks in the design direction is typically assumed to be 50\% when AADTT is given in two directions but this is not always the case. MEPDG provides 55\% as default value for level 3 analysis (1).

\section{- Percent of Trucks in the Design Lane}

The percent of trucks in the design lane or Lane Distribution Factor (LDF) considers the distribution of trucks among lines in the same direction. This factor is 1.0 for roads with two lanes or two-way highways because there is only one lane in every direction. MEPDG provides the following level 3 default values of LDF (1).

- Single-lane roadways in one direction $=1.00$

- Two-lane roadways in one direction $=0.90$

- Three-lane roadways in one direction $=0.60$

- Four-lane roadways in one direction $=0.45$

- Vehicle Operational Speed

MEPDG uses $60 \mathrm{mph}$ as default value for operational speed. This value can be modified if the designer needs to input a different value to reflect local conditions (1).

\subsubsection{Traffic Volume Adjustments}

The adjustment factors used in MEPDG for characterization and projection of traffic over pavement structures during pavement life are described as follows (1).

\section{- Monthly Adjustment Factors}

The monthly adjustment factor for a given vehicle class and month is the truck traffic of that truck class and month divided by total truck traffic for that truck class for the entire year. MEPDG assumes monthly distribution to be constant over the entire year (1). 


\section{- Truck Hourly Distribution Factors}

These factors represent the percentage of AADTT within each hour of the day. For all hierarchical levels the truck hourly distribution factor can be computed from truck traffic data measured continuously over a 24 hour period from AVC, WIM or vehicle counts. MEPDG provides default values for level 3 analysis (1).

\section{- Vehicle Class Distribution}

Vehicle class distribution data are from short duration counts such as WIM and AVC, urban traffic management center, or toll facilities. Normalized vehicle class distribution represents the percentage of each truck class in the AADTT for the base year (1). MEPDG provides default values for level 3 analysis.

\section{- Traffic Growth Factors}

The traffic growth factor is estimated from continuous traffic count data if available or from short duration counts assuming that differences from year to year are due to traffic growth (1). MEPDG provides capability to use three different traffic growth functions to compute growth or decay in truck traffic over time (1).

TABLE 2.2 MEPDG Traffic Growth Functions (NCHRP, 2004)

\begin{tabular}{|l|c|}
\hline \multicolumn{1}{|c|}{ Function Description } & Model \\
\hline No growth & $A A D T T_{X}=1.0 * A A D T T_{B Y}$ \\
\hline Linear growth & $A A D T T_{X}=G R^{*} A G E+A A D T T_{B Y}$ \\
\hline Compound growth & $A A D T T_{X}=A D T T_{B Y} *(G R)^{A G E}$ \\
\hline
\end{tabular}

where AADTT $\mathrm{X}_{\mathrm{X}}$ is the annual average daily truck traffic at age $\mathrm{X}, \mathrm{GR}$ is the traffic growth rate and $\mathrm{AADTT}_{\mathrm{BY}}$ is the base year annual average daily truck traffic.

\section{- Axle Load Distribution Factors}

MEPDG considers load intervals for each axle type, single, tandem, tridem, and quad and vehicle classification from class 4 though 13. A definition of traffic load is indicated as follow (1). 
- Single axles - 3,000 lb to 40,000 lb at 1,000-lb intervals.

- Tandem axles $-6,000 \mathrm{lb}$ to $80,000 \mathrm{lb}$ at 2,000-lb intervals.

- Tridem and quad axles $-12,000 \mathrm{lb}$ to $102,000 \mathrm{lb}$ at 3,000-lb intervals.

The load distribution factors represent the percent of total axle applications within each load interval (1). Computation of the normalized axle load distribution is only possible with WIM data thus the level of input depends on the data source, level 1 for site specific WIM data, level 2 regional WIM data, and level 3 for data from a national database (1).

\subsubsection{General Traffic Inputs}

Wheel base inputs and number of axle type by truck class are used in traffic volume calculations. The other general traffic inputs discussed in this section are related to axle load configuration and loading details used for calculations in the response models (1).

\section{- Mean Wheel Location}

Mean wheel location is the distance from the outer edge of the wheel to the pavement marking. MEPDG provides a default value of 18 inches for level 3 analysis.

\section{- Design Lane Width}

The design lane width is the distance between the lane markings on either side of the design lane. The default value provide for MEPDG is $12 \mathrm{ft}(1)$.

\section{- Number of Axle Type per Truck Class}

The number of axle type per truck class is the average number of axles for each truck class for each axle type single, tandem, tridem, and quad. The MEPDG values for level 3 analysis are presented in Table 2.3 (1). The number of quad axles is zero because only few were counted in the LTPP data base. 
TABLE 2.3 Default Values of Number of Axle Type per Truck Class (NCHRP, 2004)

\begin{tabular}{|c|c|c|c|c|}
\hline $\begin{array}{c}\text { Truck } \\
\text { Classification }\end{array}$ & $\begin{array}{c}\text { Number of Single } \\
\text { Axles per Truck }\end{array}$ & $\begin{array}{c}\text { Number of Tandem } \\
\text { Axles per Truck }\end{array}$ & $\begin{array}{c}\text { Number of Tridem } \\
\text { Axles per Truck }\end{array}$ & $\begin{array}{c}\text { Number of Quad } \\
\text { Axles per Truck }\end{array}$ \\
\hline 4 & 1.62 & 0.39 & 0.00 & 0.00 \\
\hline 5 & 2.00 & 0.00 & 0.00 & 0.00 \\
\hline 6 & 1.02 & 0.99 & 0.00 & 0.00 \\
\hline 7 & 1.00 & 0.26 & 0.83 & 0.00 \\
\hline 8 & 2.38 & 0.67 & 0.00 & 0.00 \\
\hline 9 & 1.13 & 1.93 & 0.00 & 0.00 \\
\hline 10 & 1.19 & 1.09 & 0.89 & 0.00 \\
\hline 11 & 4.29 & 0.26 & 0.06 & 0.00 \\
\hline 12 & 3.52 & 1.14 & 0.06 & 0.00 \\
\hline 13 & 2.15 & 2.13 & 0.35 & 0.00 \\
\hline
\end{tabular}

Note: The number of quad axles per truck class is 0.00 , because there were too few counted in the LTPP traffic database.

\section{- Axle Configuration}

The axle configuration can be obtained from manufacturer's databases or measured in the field. MEPDG provides typical values (1).

- Average axle-width: Distance between two outside edges of an axle. Typical value: $8.5 \mathrm{ft}$.

- Dual tire spacing: Distance between centers of a dual tire. Typical value: 12 in.

- Axle spacing: Distance between the two consecutive axles of a tandem, tridem, or quad. Typical values are 51.6 inches for tandem and 49.2 inches for tridem and quad axles.

\section{- Wheelbase}

Wheelbase refers to the distance between axles. The details of vehicle wheelbase can be measured in the field or obtained from manufacturer's databases. The typical values provided by MEPDG are indicated below (1).

- Average axle spacing

- Short: $12 \mathrm{ft}$

- Medium: $15 \mathrm{ft}$

- Long: $18 \mathrm{ft}$

\section{- Tire Dimensions and Inflation Pressure}

MEPDG provides typical values based on information collected from the trucking industry, especially from Rubber Manufacture's Association (RMA), Tire and Rim 
Association (TRA), American Trucking Association (ATA), and Truck Trailer Manufacturer's Association (TTMA). Tables 2.4 and 2.5 summarize the information (1).

TABLE 2.4 Tires Width and minimal dual tire spacing (NCHRP, 2004)

\begin{tabular}{|l|c|c|c|c|}
\hline \multirow{2}{*}{$\begin{array}{c}\text { RMA } \\
\text { Size }\end{array}$} & Ply & Minimum Dual & \multicolumn{2}{c|}{ Tire Width, in. } \\
\cline { 4 - 5 } & Rating & Spacing, in. & Section (New) & Overall (Max. Grown) \\
\hline 295/75R22.5 & 14 & 13.5 & 11.7 & 12.5 \\
\hline 11R22.5 & 14 & 12.5 & 11.0 & 12.0 \\
\hline 11 R24.5 & 14 & 12.5 & 11.0 & 12.0 \\
\hline 285/75R24.5 & 14 & 12.5 & 11.1 & 11.7 \\
\hline 11R22.5 & 16 & 12.5 & 11.0 & 12.0 \\
\hline 11R24.5 & 16 & 12.5 & 11.0 & 12.0 \\
\hline 225/70R19.5 & 12 & 10.0 & 8.9 & 9.5 \\
\hline 255/70R22.5 & 16 & 11.5 & 10.0 & 10.5 \\
\hline
\end{tabular}

TABLE 2.5 Maximum loads and cold inflation pressures (NCHRP, 2004)

\begin{tabular}{|l|c|c|c|c|c|}
\hline \multirow{2}{*}{$\begin{array}{c}\text { RMA } \\
\text { Size }\end{array}$} & \multirow{2}{*}{$\begin{array}{c}\text { Ply } \\
\text { Rating }\end{array}$} & \multicolumn{2}{c|}{ Tire Inflation Pressure, psi } & \multicolumn{2}{c|}{ Maximum Tire Load, lbs. } \\
\cline { 3 - 6 } & Single-Usage & Dual-Usage & Single-Usage & Dual-Usage \\
\hline 295/75R22.5 & 14 & 110 & 110 & 6,200 & 5,700 \\
\hline 11R22.5 & 14 & 104 & 104 & 6,200 & 5,900 \\
\hline 11R24.5 & 14 & 104 & 104 & 6,600 & 6,000 \\
\hline 285/75R24.5 & 14 & 110 & 110 & 6,200 & 5,700 \\
\hline 11R22.5 & 16 & 120 & 120 & 6,600 & 6,000 \\
\hline 11R24.5 & 16 & 120 & 120 & 7,200 & 6,600 \\
\hline 225/70R19.5 & 12 & 96 & 96 & 3,600 & 3,400 \\
\hline 255/70R22.5 & 16 & 120 & 120 & 5,500 & 5,100 \\
\hline
\end{tabular}

\subsubsection{Prediction of Total Traffic}

The total number of single, tandem, and tridem axles within each load interval is predicted with the followings steps (1).

1. Traffic growth function is used to predict the annual number of trucks per day for year $1 \mathrm{AADTT}_{1}$.

2. The total number of trucks within a time increment $i$ is calculated for month $j$ of the year $1, \mathrm{TT}_{1, \mathrm{j}, \mathrm{i}}$

$$
\mathrm{TT}_{1, \mathrm{j}, \mathrm{i}}=\left(\mathrm{AADTT}_{1}\right)\left(M D F_{j}\right)\left(\mathrm{HDF}_{\mathrm{i}}\right)(\mathrm{DDF})(\mathrm{LDF})(\# \text { of days })
$$

Where,

$\mathrm{AADTT}_{1}=$ Annual number of trucks per day for year 1

$\mathrm{MDF}_{\mathrm{j}}=$ Monthly distribution factor of month $\mathrm{j}$

$\mathrm{HDF}_{\mathrm{i}}=$ Hourly distribution factor of time increment $\mathrm{i}$

$\mathrm{DDF}=$ Directional distribution factor 
$\mathrm{LDF}=$ Lane distribution factor

3. The total number of trucks for each truck class $\left(\mathrm{T}_{1, \mathrm{j}, \mathrm{I}, \mathrm{k}}\right)$ is calculated using equation 2.59

$$
\mathrm{T}_{1, \mathrm{j}, \mathrm{I}, \mathrm{k}}=\left(\mathrm{TT}_{1, \mathrm{j}, \mathrm{i}}\right)\left(\mathrm{NTP}_{\mathrm{k}}\right)
$$

Where,

$\mathrm{TT}_{1, \mathrm{j}, \mathrm{i}}=$ Total number of trucks within a time increment (equation 2.58)

$\mathrm{NTP}_{\mathrm{k}}=$ Truck class distribution percentage for truck class $\mathrm{k}$

4. The total number of axles for each axle type a for truck class $k\left(\mathrm{NA}_{1, \mathrm{j}, \mathrm{i}, \mathrm{k}, \mathrm{a}}\right)$ is computed by equation 2.60 .

$$
\mathrm{NA}_{1, \mathrm{j}, \mathrm{i}, \mathrm{k} . \mathrm{a}}=\left(\mathrm{T}_{1, \mathrm{j}, \mathrm{i}, \mathrm{k}}\right)\left(\mathrm{NAT}_{\mathrm{k}, \mathrm{a}}\right)
$$

5. The number of axles within each load group for a specific axle type under a specific truck class $\left(\mathrm{AL}_{1, \mathrm{j}, \mathrm{i}, \mathrm{k}, \mathrm{a}, \mathrm{w}}\right)$ is calculated by equation 2.61 .

$$
\mathrm{AL}_{1, \mathrm{j}, \mathrm{i}, \mathrm{k} . \mathrm{a}, \mathrm{w}}=\left(\mathrm{NA}_{1, \mathrm{j}, \mathrm{i}, \mathrm{k} . \mathrm{a}}\right)\left(\mathrm{NWP} \mathrm{a}_{\mathrm{a}, \mathrm{w}}\right)
$$

Where,

$\mathrm{NA}_{1, \mathrm{j}, \mathrm{i}, \mathrm{k} . \mathrm{a}}=$ Total number of axles for each axle type a for truck class $\mathrm{k}$ (equation 2.60)

$\mathrm{NWP}_{\mathrm{a}, \mathrm{w}}=$ Normalized axle load distribution percentage of a specific load group

The total number of axle applications within each load group by axle type for the time increment is the sum of axle applications for each axle type, for all truck classification within each time increment.

\subsection{Materials Characterization}

The MEPDG considers the interactions between materials, climatic, traffic, and response and performance prediction models, Figure 2.7 (1). Interaction between materials, climate, traffic, response model, and performance models can be inferred from the Figure. 


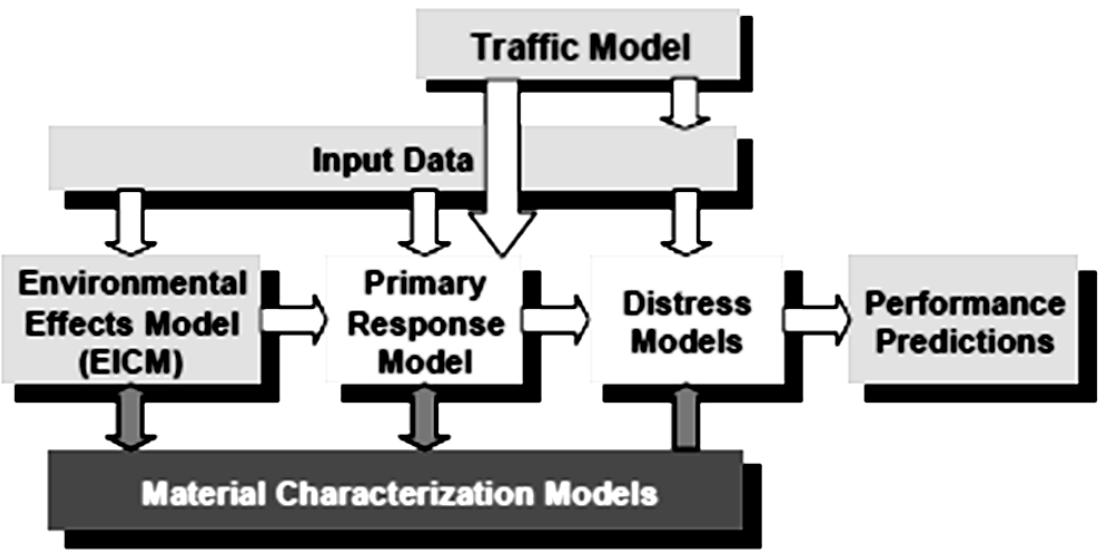

FIGURE 2.7 Interactions between Material Properties and Models (NCHRP, 2004)

The materials are categorized according to the requirements for each model as follows (1).

- Material properties required for computing pavement responses: this category includes the material properties for use in the pavement response model for computation of stress, strain, and displacements in the pavement structure.

- Materials inputs to the distress/transfer functions: this category includes the material inputs to use in the distress and smoothness models.

- Materials inputs required for climatic modeling: this category includes the material inputs to use in climatic model to determine temperature and moisture profiles in the pavement.

\subsubsection{Hierarchical Approach for Materials Inputs}

The hierarchical approach is based on the level of engineering effort exerted for characterizing the material properties. Inputs Level 1 involves comprehensive laboratory test, inputs level 2 are estimated from laboratory or field correlation, and inputs Level 3 are selected based on experience or default values. A combination of input levels is allowed according to the information available for a specific project. 


\subsubsection{Required Material Properties}

Due to the diversity of materials combinations that are used in flexible pavements, a material category grouping was developed for use in the MEPDG. With respect to flexible pavements the groups are categorized as asphalt materials, chemically stabilized, non-stabilized granular materials, subbase, soils, and bedrocks. Tables 2.2 to 2.5 present the inputs for the different group of materials. The groups are described as follows (1).

\section{- Asphalt Materials}

- Hot Mix Asphalt (HMA)—Dense Graded

- Central Plant Produced

- In-Place Recycled

- Stone Matrix Asphalt (SMA)

- Hot Mix Asphalt_-Open Graded Asphalt

- Hot Mix Asphalt-Sand Asphalt Mixtures

- Cold Mix Asphalt

- Central Plant Processed

- In-Place Recycled

\section{- Chemically Stabilized Materials}

- Cement Stabilized Aggregate

- Soil Cement

- $\quad$ Lime Cement Fly Ash

- Lime Fly Ash

- Lime Stabilized Soils

- Open Graded Cement Stabilized Aggregate

- Non-Stabilized Granular Base/Subbase

- Granular Base/Subbase 
- Sandy Subbase

- Cold Recycled Asphalt (used as aggregate)

- RAP (includes millings)

- Pulverized In-Place

- Cold Recycled Asphalt Pavement (HMA plus aggregate base/subbase)

\section{- Subgrade Soils}

- Gravelly Soils (A-1;A-2)

- Sandy Soils

- Loose Sands (A-3) and Dense Sands (A-3)

- Silty Sands (A-2-4;A-2-5)

- Clayey Sands (A-2-6; A-2-7)

- Silty Soils (A-4;A-5)

- Clayey Soils

- Low Plasticity Clays (A-6)

- Dry-Hard

- Moist Stiff

- Wet/Sat-Soft

- High Plasticity Clays (A-7)

- Dry-Hard

- Moist Stiff

- Wet/Sat-Soft

\section{- Bedrock}

- Solid, Massive and Continuous

- Highly Fractured, Weathered 
TABLE 2.6 Material Inputs for Asphalt Materials

\begin{tabular}{|c|c|c|c|c|}
\hline & \multicolumn{4}{|c|}{ MATERIAL INPUTS } \\
\hline LEVEL & MATERIAL INPUTS & & PARAMETERS & OBSERVATIONS \\
\hline \multirow{22}{*}{1} & Dynamic modulus & $\mathrm{E}^{*}$ & Dynamic modulus & $\begin{array}{l}\text { Dynamic modulus laboratory test (NCHRP 1-28A or } \\
\text { AASHTO T320) }\end{array}$ \\
\hline & & $\mathrm{G}^{*}$ & Complex shear modulus & Laboratory test (AASHTO T315) \\
\hline & & $\alpha$ & Phase angle & Laboratory test (AASHTO T315) \\
\hline & & $n$ & Binder Viscosity & $\begin{array}{l}\text { By using some conventional test or equation } 2.2 .12 \text { to } \\
\text { convert penetration to viscosity. }\end{array}$ \\
\hline & Layer Modulus for rehabilitation & $\mathrm{Ei}$ & & $\begin{array}{l}\text { Backcalculated modulus; Falling Weight Deflectometer } \\
\text { (FWD) }\end{array}$ \\
\hline & & Air void & & \multirow{4}{*}{$\begin{array}{l}\text { Laboratory test of cores, recovered binder, and recoverd } \\
\text { aggregates }\end{array}$} \\
\hline & & Asphalt Vol & & \\
\hline & & Gradation & & \\
\hline & & Asphalt viscosity & & \\
\hline & Poisson's ratio for bituminous materials & $\mu \mathrm{ac}$ & Poisson's ratio & Laboratory test (A specific test is not recommended) \\
\hline & All bituminous materials & & & $\begin{array}{l}\text { Ideally from laboratory test (Correlation or typical values } \\
\text { can be used) }\end{array}$ \\
\hline & \begin{tabular}{|l|}
-Tensile strength \\
\end{tabular} & & & Laboratory Test (AASHTO T322) \\
\hline & -Creep Compliance & & & Laboratory Test (AASHTO T322) \\
\hline & -Coefficient of Thermal Contraction & & & MEPDG includes a model (equation) \\
\hline & & LMIX & & $\begin{array}{l}\text { Linear coefficient of thermal contraction of the asphalt } \\
\text { concrete mixture }\left(1 /{ }^{\circ} \mathrm{C}\right)\end{array}$ \\
\hline & & Bac & & $\begin{array}{l}\text { Volumetric coefficient of thermal contraction of the } \\
\text { asphalt cement in the solid state }\left(1 /{ }^{\circ} \mathrm{C}\right)\end{array}$ \\
\hline & & BAGG & & $\begin{array}{l}\text { Volumetric coefficient of thermal contraction of the } \\
\text { aggregate }\left(1 /{ }^{\circ} \mathrm{C}\right)\end{array}$ \\
\hline & & VMA & & $\begin{array}{l}\text { Percent volume of voids in the mineral aggregate (equals } \\
\text { percent volume of air voids plus percent volume of } \\
\text { asphalt cement minus percent volume of absorbed } \\
\text { asphalt cement) }\end{array}$ \\
\hline & & VAGG & & Percent volume of aggregate in the mixture \\
\hline & & VTOTAL & & 100 percent \\
\hline & Surface Shortwave Absorptivity & & & $\begin{array}{l}\text { There are no current AASHTO certified standards for } \\
\text { estimating shortwave absorptivity of paving materials }\end{array}$ \\
\hline & Thermal Conductivity and Heat Capacity & & & $\begin{array}{l}\text { A direct measurement is recommended at this level } \\
\text { (ASTM E } 1952 \text { and ASTM D 2766) }\end{array}$ \\
\hline
\end{tabular}


Continuation of Table 2.6

\begin{tabular}{|c|c|c|c|c|}
\hline LEVEL & MATERIAL INPUTS & & PARAMETERS & OBSERVATIONS \\
\hline \multirow{25}{*}{2} & Master curve and shift factor: & $E^{*}$ & Dynamic modulus & Model equation \\
\hline & & $n$ & Binder Viscosity & ASTM viscosity temperature relationship \\
\hline & & G* & Complex shear modulus & Laboratory test (AASHTO T315) \\
\hline & & $\alpha$ & Phase angle & Laboratory test (AASHTO T315) \\
\hline & & $\mathrm{Va}$ & Air void content & \multirow{5}{*}{ Estimate from mix design } \\
\hline & & Vbe & Effective Asphalt Content & \\
\hline & & $\rho 34$ & $\begin{array}{l}\text { Cumulative Percent Retained } \\
\text { On } 3 / 4 \text { in Sieve }\end{array}$ & \\
\hline & & $\rho 38$ & $\begin{array}{l}\text { Cumulative Percent Retained } \\
\text { On } 3 / 8 \text { in Sieve }\end{array}$ & \\
\hline & & $\rho 4$ & $\begin{array}{l}\text { Cumulative Percent Retained } \\
\text { On \#4 Sieve }\end{array}$ & \\
\hline & & 200 & Percent Passing \#200 Sieve & \\
\hline & Layer Modulus for rehabilitation & $\mathrm{Ei}$ & & Predictive equation \\
\hline & & Air void & & from cores \\
\hline & & Asphalt Vol & & from cores \\
\hline & & Gradation & & from cores \\
\hline & & Asphalt viscosity & & from cores \\
\hline & & Mri & indirect resilient modulus & $\begin{array}{l}\text { laboratory tests using revised protocol developed } \\
\text { at University of Maryland for NCHRP 1-28A from field } \\
\text { cores }\end{array}$ \\
\hline & $\begin{array}{l}\text { Poisson's ratio for bituminous } \\
\text { materials }\end{array}$ & $\mu a c$ & Poisson's ratio & \\
\hline & Hot mix asphalt - Dense graded & & & Equations or typical values \\
\hline & $\begin{array}{l}\text { Open-Graded Asphalt Treated } \\
\text { Materials }\end{array}$ & & & Typical values \\
\hline & Cold-Mix Asphalt (CMA) Materials & & & Typical values \\
\hline & Tensile strength. & & & Laboratory Test (AASHTO T322) \\
\hline & Creep Compliance & & & Laboratory Test (AASHTO T322) \\
\hline & Coefficient of Thermal Contraction & & & Use equation 2.2.27 same parameter as in level 1 \\
\hline & Surface Shortwave Absorptivity & & & $\begin{array}{l}\text { Use typical values, Weathered asphalt (gray) } 0.80-0.90, \\
\text { Fresh asphalt (black) } 0.90-0.98\end{array}$ \\
\hline & \multicolumn{2}{|l|}{ Thermal Conductivity and Heat Capacity } & & Use typical values \\
\hline
\end{tabular}


Continuation of Table 2.6

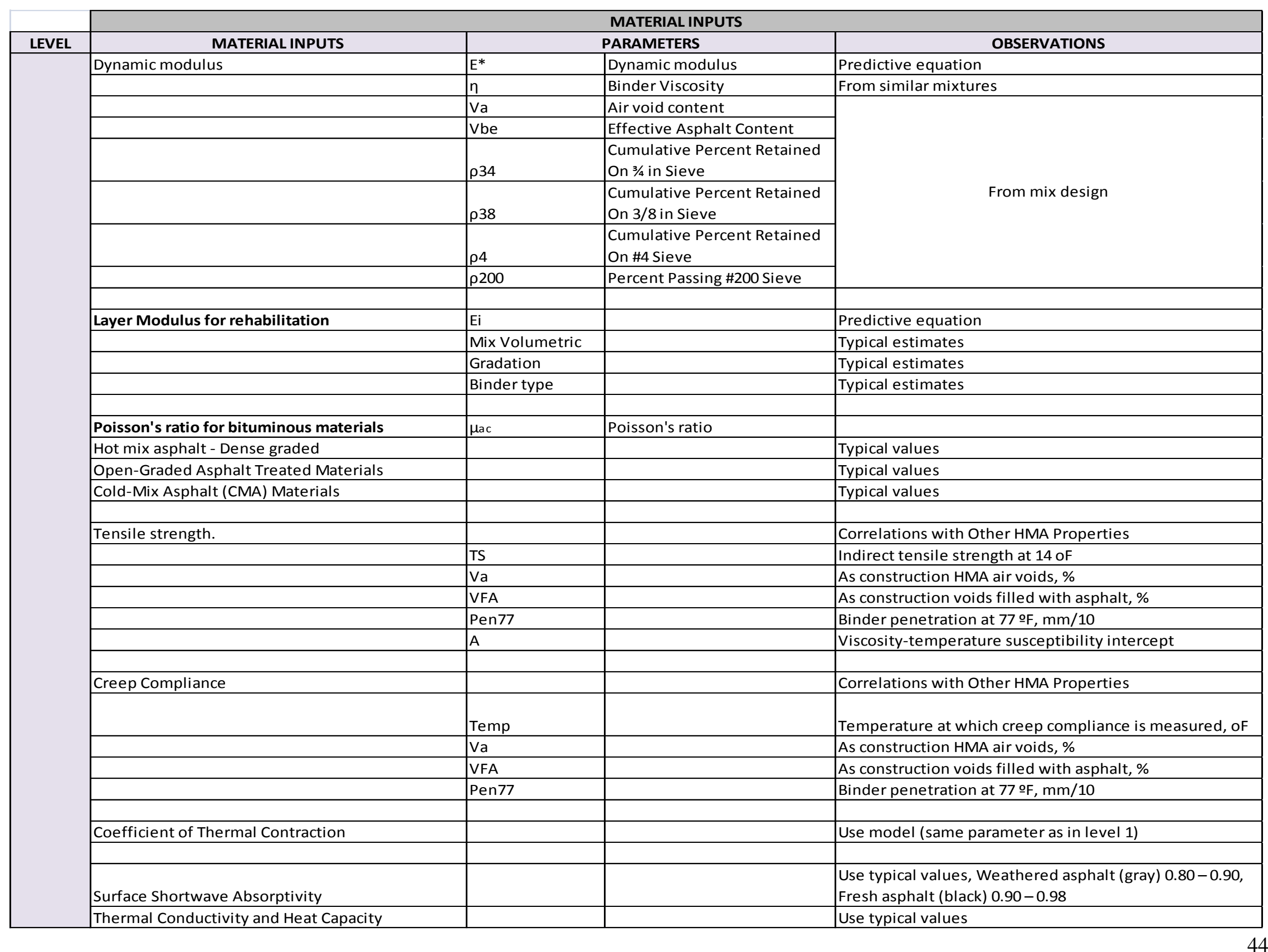


TABLE 2.7 Material Inputs for Chemically Stabilized Materials

\begin{tabular}{|c|c|c|c|}
\hline \multirow[b]{2}{*}{ LEVEL } & \multicolumn{3}{|c|}{ MATERIAL INPUTS } \\
\hline & MATERIAL INPUTS & PARAMETERS & OBSERVATIONS \\
\hline \multirow{25}{*}{1} & Elastic Modulus or Resilient Modulus for Design & E or Mr & $\begin{array}{l}\text { E is required for lean concrete, cement stabilized, open } \\
\text { graded cement stabilized materials, soil cement, lime- } \\
\text { cement-flyash; Mr is Required for lime stabilized soils }\end{array}$ \\
\hline & Lean concrete & E & Laboratory test, ASTM C 469 \\
\hline & Cement treated aggregate & $\mathrm{E}$ & Laboratory test, ASTM C 469 \\
\hline & Open graded cement stabilized & $\mathrm{E}$ & $\begin{array}{l}\text { Modulus testing at level } 1 \text { is not possible due to } \\
\text { lack of standard test protocols }\end{array}$ \\
\hline & Lime-cement-flyash & $\mathrm{E}$ & $\begin{array}{l}\text { Modulus testing at level } 1 \text { is not possible due to } \\
\text { lack of standard test protocols }\end{array}$ \\
\hline & Soil cement & $\mathrm{E}$ & $\begin{array}{l}\text { Modulus testing at level } 1 \text { is not possible due to } \\
\text { lack of standard test protocols }\end{array}$ \\
\hline & Lime stabilized soils & $\mathrm{Mr}$ & $\begin{array}{l}\text { Mixture Design and Testing Protocol (MDTP) in } \\
\text { conjunction with the AASHTO T307 test protocol }\end{array}$ \\
\hline & The Modulus for Design for in-service pavements & $\mathrm{E}$ & The moduli can be determined from the FWD \\
\hline & Flexural Strength for Design & MR & $\begin{array}{l}\text { Flexural Strength for Design is required for HMA } \\
\text { Pavement Only }\end{array}$ \\
\hline & Lean concrete & MR & AASHTO T97 \\
\hline & Cement treated aggregate & MR & AASHTO T97 \\
\hline & Open graded cement stabilized & MR & Not available \\
\hline & \begin{tabular}{|l|} 
Lime-cement-flyash \\
\end{tabular} & MR & AASHTO T97 \\
\hline & Soil cement & MR & ASTM D 1635 \\
\hline & Lime stabilized soils & MR & $\begin{array}{l}\text { No current AASHTO or ASTM tests available. } \\
\text { Therefore, level } 1 \text { testing is not recommended. }\end{array}$ \\
\hline & Poisson's Ratio for Design & $\mu$ & $\begin{array}{l}\text { Although this parameter can be determined from } \\
\text { laboratory testing the cost and time required may not be } \\
\text { justified }\end{array}$ \\
\hline & \begin{tabular}{|l|} 
Lean concrete \\
\end{tabular} & $\mu$ & \multirow{6}{*}{$\begin{array}{l}\text { Typical values may be used for new, reconstruction, and } \\
\text { rehabilitation design with overlays }\end{array}$} \\
\hline & Cement treated aggregate & $\mu$ & \\
\hline & \begin{tabular}{|l|} 
Open graded cement stabilized \\
\end{tabular} & $\mu$ & \\
\hline & Lime-cement-flyash & $\mu$ & \\
\hline & Soil cement & $\mu$ & \\
\hline & Lime stabilized soils & $\mu$ & \\
\hline & Thermal Conductivity and Heat Capacity for Design & & \\
\hline & Thermal Conductivity & $\mathrm{K}$ & $\begin{array}{l}\text { A direct measurement is recommended at this level } \\
\text { (ASTM E 1952) }\end{array}$ \\
\hline & Heat Capacity & Q & $\begin{array}{l}\text { A direct measurement is recommended at this level } \\
\text { (ASTM D } 2766\end{array}$ \\
\hline
\end{tabular}


Continuation of table Table 2.7

\begin{tabular}{|c|c|c|c|}
\hline \multirow[b]{2}{*}{ LEVEL } & \multicolumn{3}{|c|}{ MATERIAL INPUTS } \\
\hline & MATERIAL INPUTS & PARAMETERS & OBSERVATIONS \\
\hline \multirow{24}{*}{2} & Elastic Modulus or Resilient Modulus for Design & E or Mr & \\
\hline & Lean concrete & $\mathrm{E}$ & $\begin{array}{l}\text { Correlation with f'c compressive strength; tested in } \\
\text { accordance with AASHTO T22 }\end{array}$ \\
\hline & Cement treated aggregate & $\mathrm{E}$ & $\begin{array}{l}\text { Correlation with f'c compressive strength; tested in } \\
\text { accordance with AASHTO T22 }\end{array}$ \\
\hline & Open graded cement stabilized & $\mathrm{E}$ & No correlations are available \\
\hline & Lime-cement-flyash & E & $\begin{array}{l}\text { Correlation with qu = unconfined compressive strength, } \\
\text { psi tested in accordance with ASTM C } 593\end{array}$ \\
\hline & Soil cement & $\mathrm{E}$ & $\begin{array}{l}\text { Correlation with qu = unconfined compressive strength, } \\
\text { psi tested in accordance with ASTM D } 1633\end{array}$ \\
\hline & Lime stabilized soils & $\mathrm{Mr}$ & $\begin{array}{l}\text { Correlation with qu = unconfined compressive strength, } \\
\text { psi tested in accordance with ASTM D } 5102\end{array}$ \\
\hline & Flexural Strength for Design & MR & $\begin{array}{l}\text { MR can be estimated from correlation with unconfined } \\
\text { compressive strength (qu) testing of the cured chemically } \\
\text { stabilized material samples }\end{array}$ \\
\hline & Lean concrete & MR & $\begin{array}{l}\text { AASHTO T22; MR can be conservatively estimated as } \\
\text { being } 20 \text { percent of the qu }\end{array}$ \\
\hline & Cement treated aggregate & MR & $\begin{array}{l}\text { AASHTO T22;MR can be conservatively estimated as } \\
\text { being } 20 \text { percent of the qu }\end{array}$ \\
\hline & Open graded cement stabilized & $\mathrm{MR}$ & Not available \\
\hline & Lime-cement-flyash & MR & $\begin{array}{l}\text { ASTM C 593; MR can be conservatively estimated as } \\
\text { being } 20 \text { percent of the qu }\end{array}$ \\
\hline & Soil cement & MR & $\begin{array}{l}\text { ASTM D 1633; MR can be conservatively estimated as } \\
\text { being } 20 \text { percent of the qu }\end{array}$ \\
\hline & Lime stabilized soils & MR & $\begin{array}{l}\text { ASTM D 5102; MR can be conservatively estimated as } \\
\text { being } 20 \text { percent of the qu }\end{array}$ \\
\hline & Poisson's Ratio for Design & $\mu$ & $\begin{array}{l}\text { Although this parameter can be determined from } \\
\text { laboratory testing the cost and time required may not be } \\
\text { justified }\end{array}$ \\
\hline & Lean concrete & $\mu$ & \multirow{6}{*}{$\begin{array}{l}\text { Typical values may be used for new, reconstruction, and } \\
\text { rehabilitation design with overlays }\end{array}$} \\
\hline & Cement treated aggregate & $\mu$ & \\
\hline & Open graded cement stabilized & $\mu$ & \\
\hline & Lime-cement-flyash & $\mu$ & \\
\hline & Soil cement & $\mu$ & \\
\hline & Lime stabilized soils & $\mu$ & \\
\hline & Thermal Conductivity and Heat Capacity for Design & & \\
\hline & Thermal Conductivity & $\mathrm{K}$ & Not applicable. \\
\hline & Heat Capacity & Q & Not applicable. \\
\hline
\end{tabular}


Continuation of table Table 2.7

\begin{tabular}{|c|c|c|c|}
\hline \multirow[b]{2}{*}{ LEVEL } & \multicolumn{3}{|c|}{ MATERIAL INPUTS } \\
\hline & \begin{tabular}{|c|} 
MATERIAL INPUTS \\
\end{tabular} & PARAMETERS & OBSERVATIONS \\
\hline \multirow{24}{*}{3} & Elastic Modulus or Resilient Modulus for Design & $\mathrm{E}$ or $\mathrm{Mr}$ & \\
\hline & Lean concrete & $E$ & \\
\hline & Cement treated aggregate & E & Estimated from experience or historical records or typical \\
\hline & Open graded cement stabilized & $\mathrm{E}$ & values \\
\hline & Lime-cement-flyash & $\mathrm{E}$ & There is a minimum value required for HMA pavements \\
\hline & Soil cement & $\mathrm{E}$ & only \\
\hline & Lime stabilized soils & $\mathrm{Mr}$ & \\
\hline & Flexural Strength for Design & MR & $\begin{array}{l}\text { MR is estimated from experience or historical records } \\
\text { based on material description }\end{array}$ \\
\hline & Lean concrete & MR & \multirow{6}{*}{ Typical values } \\
\hline & Cement treated aggregate & MR & \\
\hline & Open graded cement stabilized & MR & \\
\hline & Lime-cement-flyash & $\mathrm{MR}$ & \\
\hline & Soil cement & MR & \\
\hline & Lime stabilized soils & MR & \\
\hline & Poisson's Ratio for Design & $\boldsymbol{\mu}$ & $\begin{array}{l}\text { Although this parameter can be determined from } \\
\text { laboratory testing the cost and time required may not be } \\
\text { justified }\end{array}$ \\
\hline & Lean concrete & $\mu$ & \multirow{6}{*}{$\begin{array}{l}\text { Typical values may be used for new, reconstruction, and } \\
\text { rehabilitation design with overlays }\end{array}$} \\
\hline & Cement treated aggregate & $\mu$ & \\
\hline & Open graded cement stabilized & $\mu$ & \\
\hline & Lime-cement-flyash & $\mu$ & \\
\hline & Soil cement & $\mu$ & \\
\hline & Lime stabilized soils & $\mu$ & \\
\hline & Thermal Conductivity and Heat Capacity for Design & & \\
\hline & Thermal Conductivity & $\mathrm{K}$ & \multirow{2}{*}{$\begin{array}{l}\text { User selects design values based upon agency historical } \\
\text { data or from typical values }\end{array}$} \\
\hline & Heat Capacity & Q & \\
\hline
\end{tabular}


TABLE 2.8 Material Inputs for Non-stabilized Base, Subbase, and Subgrde Soils

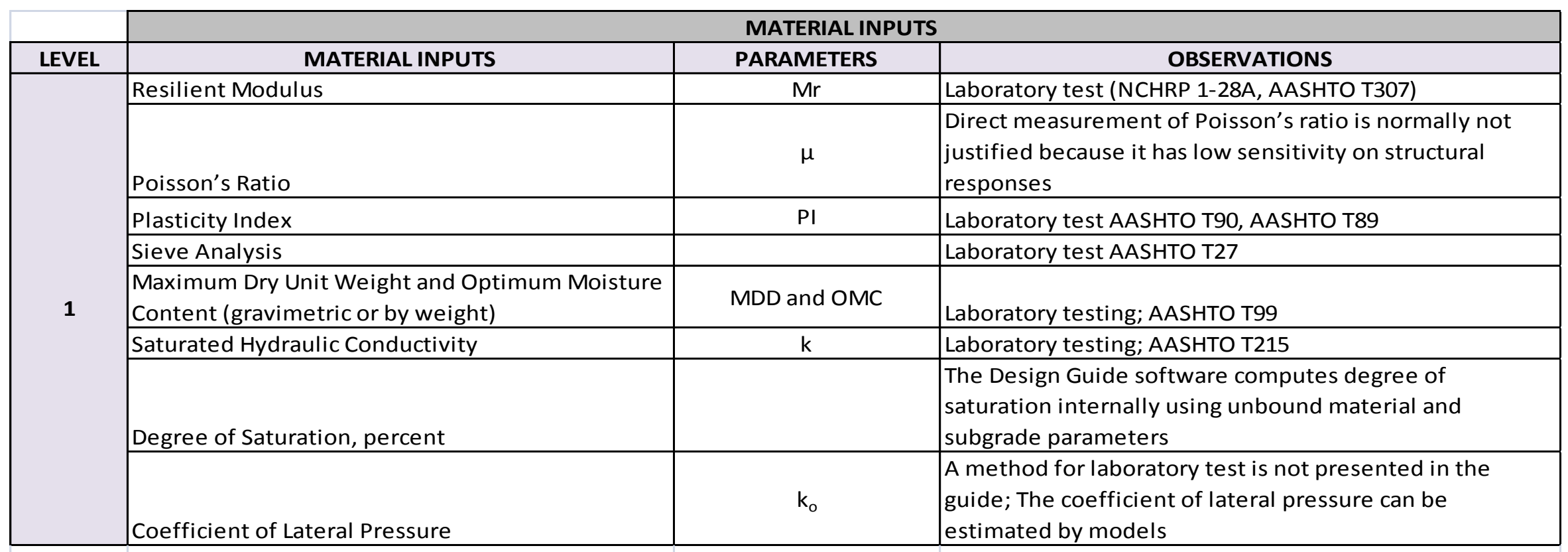


Continuation Table 2.8

\begin{tabular}{|c|c|c|c|}
\hline & \multicolumn{3}{|c|}{ MATERIAL INPUTS } \\
\hline LEVEL & MATERIAL INPUTS & PARAMETERS & OBSERVATIONS \\
\hline \multirow{13}{*}{2} & Resilient Modulus & $\mathrm{Mr}$ & Correlation with other properties \\
\hline & & CBR & CBR = California Bearing Ratio, percent; AASHTO T193 \\
\hline & & R-value & $\begin{array}{l}\text { Resistance Rvalue and Expansion Pressure of } \\
\text { Compacted Soils; AASHTO T190 }\end{array}$ \\
\hline & & $\begin{array}{l}\text { AASHTO layer } \\
\text { coefficient }\end{array}$ & AASHTO Guide for the Design of Pavement Structures \\
\hline & & $\begin{array}{l}\mathrm{PI} \text { and } \\
\text { gradation }\end{array}$ & $\begin{array}{l}\text { AASHTO T27. "Sieve Analysis of Coarse and Fine } \\
\text { Aggregates" } \\
\text { AASHTO T90, "Determining the Plastic Limit and Plasticity } \\
\text { Index of Soils" }\end{array}$ \\
\hline & & DCP & $\begin{array}{l}\text { Dynamic Cone Penetrometer in Shallow Pavement } \\
\text { Applications; ASTM D } 6951\end{array}$ \\
\hline & Poisson's Ratio & $\mu$ & $\begin{array}{l}\text { Adopt models and correlations based on local knowledge } \\
\text { and experience }\end{array}$ \\
\hline & Plasticity Index & $\mathrm{PI}$ & $\begin{array}{l}\text { Input for this Parameter as only test values (level 1) are } \\
\text { recommended; AASHTO T90, AASHTO T89 }\end{array}$ \\
\hline & Sieve Analysis & & Laboratory test AASHTO T27 \\
\hline & $\begin{array}{l}\text { Maximum Dry Unit Weight and Optimum Moisture } \\
\text { Content (gravimetric or by weight) }\end{array}$ & MDD and OMC & $\begin{array}{l}\text { MDD and OMC are estimated using correlations or } \\
\text { model }\end{array}$ \\
\hline & Saturated Hydraulic Conductivity & $k$ & $\begin{array}{l}\text { Computed internally by the software using the } \mathrm{PI} \text { and } \\
\text { gradation information }\end{array}$ \\
\hline & Degree of Saturation, percent & & $\begin{array}{l}\text { The Design Guide software computes degree of } \\
\text { saturation internally using unbound material and } \\
\text { subgrade parameters }\end{array}$ \\
\hline & Coefficient of Lateral Pressure & $\mathrm{k}_{\mathrm{o}}$ & $\begin{array}{l}\text { The coefficient of lateral pressure can be estimated } \\
\text { by models }\end{array}$ \\
\hline
\end{tabular}


Continuation Table 2.8

\begin{tabular}{|c|c|c|c|}
\hline & \multicolumn{3}{|c|}{ MATERIAL INPUTS } \\
\hline \multirow{6}{*}{3} & Poisson's Ratio & $\mu$ & Typical values \\
\hline & Sieve Analysis & & Laboratory test AASHTO T27 \\
\hline & $\begin{array}{l}\text { Maximum Dry Unit Weight and Optimum Moisture } \\
\text { Content (gravimetric or by weight) }\end{array}$ & MDD and OMC & $\begin{array}{l}\text { Values are assumed based on local experience. MDD } \\
\text { typically ranges from } 100 \text { to } 140 \text { pcf while OMC ranges } \\
\text { from } 4 \text { to } 15 \text { percent }\end{array}$ \\
\hline & Saturated Hydraulic Conductivity & $\mathrm{k}$ & Not applicable \\
\hline & Degree of Saturation, percent & & $\begin{array}{l}\text { The Design Guide software computes degree of } \\
\text { saturation internally using unbound material and } \\
\text { subgrade parameters }\end{array}$ \\
\hline & Coefficient of Lateral Pressure & $\mathrm{k}_{\mathrm{o}}$ & $\begin{array}{l}\text { The coefficient of lateral pressure can be estimated } \\
\text { by models }\end{array}$ \\
\hline
\end{tabular}


TABLE 2.9 Material Inputs for Bedrock

\begin{tabular}{|c|c|c|c|}
\hline & \multicolumn{3}{|c|}{ MATERIAL INPUTS } \\
\hline LEVEL & MATERIAL INPUTS & PARAMETERS & OBSERVATIONS \\
\hline \multirow{5}{*}{1} & $\begin{array}{l}\text { Modulus of Elasticity of Bedrock Materials for new, } \\
\text { reconstruction and rehabilitation design }\end{array}$ & $\mathrm{E}$ & $\begin{array}{l}\text { This level is not considered applicable for } \\
\text { bedrock conditions }\end{array}$ \\
\hline & Poisson's Ratio of Bedrock Materials & $\mu$ & This parameter is rarely measured and is often assumed \\
\hline & & & \\
\hline & & & \\
\hline & \multicolumn{3}{|c|}{ MATERIAL INPUTS } \\
\hline LEVEL & MATERIAL INPUTS & PARAMETERS & OBSERVATIONS \\
\hline \multirow{5}{*}{2} & $\begin{array}{l}\text { Modulus of Elasticity of Bedrock Materials for new, } \\
\text { reconstruction and rehabilitation design }\end{array}$ & $\mathrm{E}$ & $\begin{array}{l}\text { This level is not considered applicable for } \\
\text { bedrock conditions }\end{array}$ \\
\hline & Poisson's Ratio of Bedrock Materials & $\mu$ & Use typical Poisson's ratio values \\
\hline & & & \\
\hline & & & \\
\hline & \multicolumn{3}{|c|}{ MATERIAL INPUTS } \\
\hline LEVEL & MATERIAL INPUTS & PARAMETERS & OBSERVATIONS \\
\hline \multirow{2}{*}{3} & $\begin{array}{l}\text { Modulus of Elasticity of Bedrock Materials for new, } \\
\text { reconstruction and rehabilitation design }\end{array}$ & $\mathrm{E}$ & $\begin{array}{l}\text { This level is not considered applicable for } \\
\text { bedrock conditions }\end{array}$ \\
\hline & Poisson's Ratio of Bedrock Materials & $\mu$ & Use typical Poisson's ratio values \\
\hline
\end{tabular}




\section{CHAPTER 3: PREVIOUS SENSITIVITY ANALYSIS OF MEPDG}

\subsection{Pilot Study in Sampling-based Sensitivity Analysis of MEPDG}

Graves and Mahboub, 2006, analyzed the sensitivity of several parameters for flexible pavements in the MEPDG using Monte Carlo sampling over the entire input space for the following parameters: nominal maximum aggregate size (NMAS), climate location, HMA thickness, AADTT, subgrade strength, truck traffic category, construction season, and binder grade. The individual values utilized in the study are given in Table 3.1 (7).

TABLE 3.1 Individual values utilized in pilot study (Graves and Mahboub, 2006).

\begin{tabular}{|c|c|c|c|c|c|c|}
\hline AADTT & $\begin{array}{c}\text { Truck Traffic } \\
\text { Classification }\end{array}$ & $\begin{array}{c}\text { HMA } \\
\text { Thickness (in) }\end{array}$ & $\begin{array}{c}\text { Subgrade } \\
\text { CBR }\end{array}$ & NMAS & $\begin{array}{c}\text { Climate } \\
\text { Zone }\end{array}$ & $\begin{array}{c}\text { Construction } \\
\text { Month }\end{array}$ \\
\hline 100 & 1 & 5 & 2 & 19 & Cheyenne & January \\
500 & 4 & 6 & 4 & 25 & Phoenix & April \\
1000 & 6 & 7 & 6 & 37.5 & Lexington & July \\
2000 & 12 & 8 & 8 & & Birmingham & October \\
4000 & & 9 & 10 & & & \\
6000 & & 10 & & & & \\
8000 & & 11 & & & & \\
10000 & & 12 & & & & \\
15000 & & 13 & & & & \\
25000 & & 14 & & & & \\
\hline
\end{tabular}

A total of 100 design sections were run and the resulting predicted performance of longitudinal cracking, HMA and total rutting, and IRI were analyzed by Pearson's and Spearman's correlation coefficients. These correlation coefficients provide a means to evaluate relative sensitive of a given input to an output of interest. A negative correlation coefficient indicates that as the input parameter increases the output decreases, whereas a positive correlation coefficient indicates that as the input parameter increases the output increases. Charts were produced for each parameter in the study. Figure 3.1 shows an example of those charts; correlations mark with a star are significant at $95 \%$ confidence level. These statistics and the relative rank of the individual parameters from Pearson's and Spearman's correlation coefficients were used to evaluate the input parameters. 
The conclusions from this study indicated that AADTT, HMA thickness, and subgrade strength have a significant impact on performance, whereas the remaining parameters have lesser impact. The authors claim that this type of sensitivity analysis is adequate to identify important parameters in the MEPDG (7).

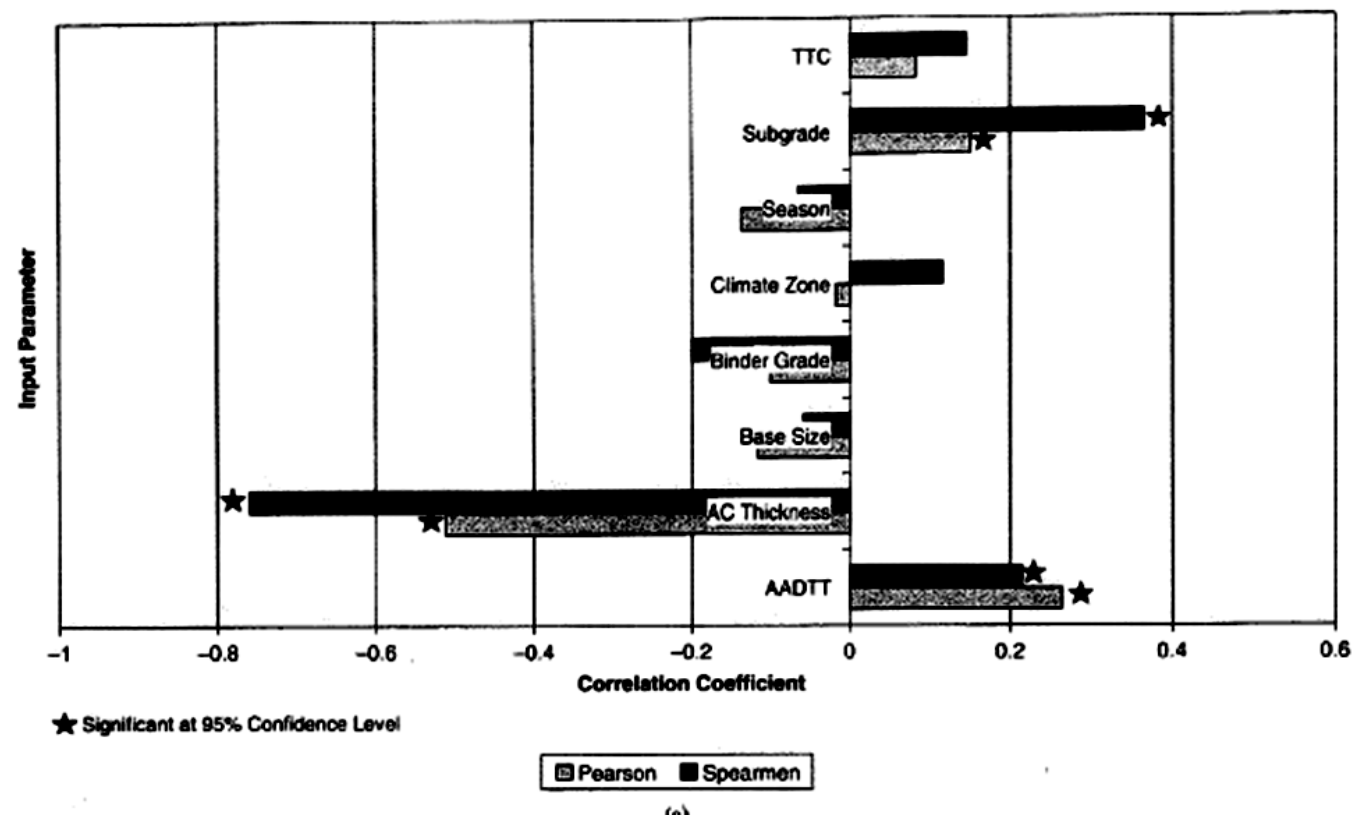

FIGURE 3.1 Pearson's and Spearman's Coefficients (Graves and Mahboub, 2006)

\subsection{Sensitivity of Design Input Variables in MEPDG for Rigid Pavements}

Hall and Beam, 2005, performed a study of sensitivity of design input variables for rigid pavements in MEPDG. They demonstrated that the MEPDG has more than 100 inputs of traffic, environmental condition, and materials to model rigid pavement performance over its design life. This study evaluated 29 input variables by analyzing a standard pavement section and changing the values of each input one at time while having the other inputs fixed. The effect of the changes on the pavement distress model for cracking, faulting, and roughness were analyzed. In a first step, the study analyzed a standard pavement structure by varying one design input per trial to show sensitivity of the system to that particular input. Then, the inputs that have a significant effect on the overall performance were determined. Finally, the reasonableness of any trend of distress models was evaluated. 
The general information, traffic, and some structural parameters were treated as constants in the analysis. Table 3.2 displays the structural inputs that were treated as constant. The 29 inputs that were varied in the study and their sensitivity regarding cracking, faulting, and smoothness are shown in Table 3.3. In general, the MEPDG models were not sensitive to 17 of the 29 input in the study. All three models were sensitive to six of the 29 inputs, combinations of only one or two of the distress models were sensitive to six of the 29 inputs in study (8).

TABLE 3.2 Structural Constant Inputs in the Study (Hall and Beam, 2005)

\begin{tabular}{|c|c|}
\hline Project Information & Baseline Value \\
\hline \multicolumn{2}{|l|}{ Layer 2-unbound material } \\
\hline $\begin{array}{l}\text { Unbound material } \\
\text { Thickness (in.) }\end{array}$ & $\begin{array}{l}\text { Crushed stone } \\
12\end{array}$ \\
\hline Strength properties: Level 3 & \\
\hline $\begin{array}{l}\text { Poisson's ratio } \\
\text { Coefficient of lateral pressure, Ko } \\
\text { Modulus (input) (psi) }\end{array}$ & $\begin{array}{l}0.35 \\
0.5 \\
40,000\end{array}$ \\
\hline \multicolumn{2}{|l|}{ Analysis type: ICM inputs } \\
\hline $\begin{array}{l}\text { Plasticity index (PI) } \\
\text { Passing \#200 sieve (\%) } \\
\text { Passing *4 sieve (\%) } \\
\text { D60 (mm) } \\
\text { Dry thermal conductivity (BTU/h-ft- }{ }^{\circ} \mathrm{F} \text { ) } \\
\text { Dry heat capacity (BTU/nb-॰ F) } \\
\text { Maximum dry unit weight (pci) (user input) } \\
\text { Specific gravity of solids (user input) } \\
\text { Saturated hydraulic conductivity (ft/h) (user input) } \\
\text { Optimum gravimetric water content (\%) (user input) } \\
\text { Calculated de gree of saturation (\%) }\end{array}$ & $\begin{array}{l}1 \\
10 \\
80 \\
2 \\
0.23 \\
0.17 \\
120 \\
2.67 \\
1 \\
11.2 \\
82.8\end{array}$ \\
\hline \multicolumn{2}{|l|}{ Soll-water characteristic curve parameters } \\
\hline $\begin{array}{l}\text { a } \\
\text { b } \\
\text { c } \\
\text { h }\end{array}$ & $\begin{array}{l}11.4 \\
1.72 \\
0.518 \\
371\end{array}$ \\
\hline
\end{tabular}

\begin{tabular}{|c|c|}
\hline Layer 3-subgrade & Baseline Value \\
\hline $\begin{array}{l}\text { Material type } \\
\text { Thickness }\end{array}$ & $\begin{array}{l}\text { AASHTO } \\
\text { Class A-6 } \\
\text { Semi-infinite }\end{array}$ \\
\hline \multicolumn{2}{|l|}{ Strength properties: Level 3} \\
\hline $\begin{array}{l}\text { Poisson's ratio } \\
\text { Coefficient of lateral pressure, Ko } \\
\text { Modulus (input) (psi) }\end{array}$ & $\begin{array}{l}0.35 \\
0.5 \\
18,000\end{array}$ \\
\hline \multicolumn{2}{|l|}{ Aralysis type: ICM inputs } \\
\hline $\begin{array}{l}\text { Plasticity index (PI) } \\
\text { Passing \#200 sieve (\%) } \\
\text { Passing } \% 4 \text { sieve (\%) } \\
\text { D60 (mum) } \\
\text { Dry thermal conductivity (BTU/h- } \mathrm{ft}-{ }^{-} \mathrm{F} \text { ) } \\
\text { Dry heat capacity (BTU/b--F) } \\
\text { Maximum dry unit weight (pef) (user input) } \\
\text { Specific gravity of solids (user input) } \\
\text { Saturated hydraulic conductivity (fth) (user input) } \\
\text { Optimum gravimetric water content (\%) (user input) } \\
\text { Calculated degree of saturation (\%) }\end{array}$ & $\begin{array}{l}25 \\
80 \\
95 \\
0.01 \\
0.23 \\
0.17 \\
100.8 \\
2.75 \\
6.52 \mathrm{e}-007 \\
22.6 \\
88.5\end{array}$ \\
\hline \multicolumn{2}{|l|}{ Soil-water characteristic curve parameters } \\
\hline $\begin{array}{l}a \\
b \\
c \\
b\end{array}$ & $\begin{array}{l}174 \\
1.05 \\
0.707 \\
8190\end{array}$ \\
\hline
\end{tabular}

Ko $=$ coefficient of lateral earth preasure; ICM = Integrated Climatic Model; D60 = grain size at which $60 \%$ of material is finer, and a, b, $\mathrm{c}$, and $\mathrm{h}=$ soilwater characteristic curve coefficients. 
TABLE3.3 Results of Sensitivity Analysis (Hall and Beam, 2005)

\begin{tabular}{|c|c|c|c|}
\hline \multirow[b]{2}{*}{ JPCP Concrete Material Characteristics } & \multicolumn{3}{|c|}{ Performance Models } \\
\hline & Faulting & Cracking & Smoothness \\
\hline Cur-warp effective temperature difference & $\mathbf{S}$ & $\mathbf{S}$ & $\mathbf{s}$ \\
\hline Joint spacing & $\mathbf{S}$ & $\$$ & $\mathbf{S}$ \\
\hline Sealant type & I & I & I \\
\hline Dowell diancter & $\mathbf{s}$ & $\mathbf{I}$ & $\mathbf{S}$ \\
\hline Dowell spacing . & $\mathbf{I}$ & $\mathbf{I}$ & 1 \\
\hline Edge suppont & $\mathbf{S}$ & $\mathbf{s}$ & $\mathbf{S}$ \\
\hline PCC-base interface & $\mathbf{I}$ & $\mathbf{I}$ & $\mathbf{I}$ \\
\hline Erodibility index & I & 1 & $\mathbf{I}$ \\
\hline Surface shoutwave absorptivity & $\mathbf{I}$ & $\mathbf{s}$ & 1 \\
\hline Infiltration of surface water & I & $\mathbf{I}$ & I \\
\hline Drainage path length & I & 1 & $l$ \\
\hline Pavement eross-slope & $\mathbf{I}$ & 1 & l \\
\hline PCC layer thickness & $\mathbf{s}$ & $\mathbf{s}$ & $\mathbf{S}$ \\
\hline Unit weight & $\mathbf{S}$ & $\mathbf{S}$ & $\mathbf{s}$ \\
\hline Poisson's ratio & 1 & $\mathbf{S}$ & I \\
\hline Coefficient of thermal expansion & $\mathbf{s}$ & $\mathbf{s}$ & $\mathbf{S}$ \\
\hline Thermal conductivity & 1 & $\mathbf{s}$ & $\mathbf{l}$ \\
\hline Heat capacily & I & $\mathbf{I}$ & I \\
\hline Cement type & 1 & I & $\mathbf{I}$ \\
\hline Cement content & $\mathbf{I}$ & $\mathbf{J}$ & I \\
\hline Water/cement ratio & $\mathbf{I}$ & $\mathbf{I}$ & $\mathbf{I}$ \\
\hline Aggregate type & $\mathbf{I}$ & 1 & I \\
\hline PCC set temperature & $\mathbf{I}$ & $\mathbf{I}$ & 1 \\
\hline Ultimate shrinkage at $40 \%$ R.H. & $\mathbf{I}$ & 1 & 1 \\
\hline Reversible shrinkage & $\mathbf{I}$ & $\mathbf{I}$ & I \\
\hline Time to develop $50 \%$ of ultimate shrinkage & $\mathbf{I}$ & 1 & I \\
\hline Curing method & $\mathbf{I}$ & I & $\mathbf{I}$ \\
\hline 28-day PCC modulus of rupture & $\mathbf{I}$ & $\mathbf{S}$ & $\mathbf{S}$ \\
\hline 28-day PCC compressive strength & 1 & $\mathbf{S}$ & $\mathbf{S}$ \\
\hline
\end{tabular}

\subsection{Sensitivity of MEPDG to Traffic Inputs}

Papagiannakis, et al, 2006, investigated the sensitivity of the MEPDG to traffic input. They selected 30 sites from the LTPP database (15 flexible and 15 rigid pavements) and simulated 17 traffic scenarios consisting of combination of site specific, regional, and national data including total truck counts, truck class and axle load distribution. The pavement life was defined as the length of time required for one of the distress parameters to reach the critical threshold level (rutting and longitudinal cracking for flexible pavements and cracking and punchouts for rigid pavements). The data were used in the MEPDG to obtain the maximum value in pavement life predictions given a confidence level. The reliability in the pavement design process reflects the confidence that this level of error will not be exceeded. The study indicated 
that where continuous weight in motion (WIM) data are used the error in predicting pavement life is lower than $10 \%, 16 \%$, and $27 \%$ for confidence levels of $75 \%, 85 \%$, and $95 \%$ respectively. Where site-specific truck counts are combined with regional load and classification data, life prediction errors may range from $25 \%$ to $64 \%$. Where continuous site-specific truck counts are combined with regional classification and national load data, life prediction errors may range from $27 \%$ to $68 \%$. Where site-specific truck counts are combined with national load and classification data, life prediction error may range from $30 \%$ to $76 \%$ (9).

\subsection{Simplified Approach for Sensitivity of Rigid Pavement Inputs in the MEPDG}

Haider, et al, 2009, conducted a sensitivity analysis over some variables of rigid pavements in the MEPDG. They performed the analysis with two main steps: first, authors identified the 23 most sensitive parameters by varying one parameter at time over defined input ranges. Second, the authors performed a full factorial experiment in an attempt to identify interactions between the most sensitive parameters. Due to the large number of runs required to perform the full factorial experiment over the 23 sensitive parameters, each at 3 levels $\left(3^{23}=\right.$ $9.4 \times 10^{10}$ runs), the number of parameters included in the factorial experiment was reduced to 6 parameters at 2 levels and 1 environmental parameter at 3 levels $\left(2^{6}\right.$ x $3=192$ runs). The reduction of parameters was accomplished based on engineering judgment and local experience of Michigan Department of Transportation. The results showed that the effect of PCC thickness, joint spacing and edge support have significant effects among design parameters while coefficient of thermal expansion, modulus of rupture, base type and subgrade were significant among the material-related properties (10). 


\section{CHAPTER 4: DESIGN OF EXPERIMENTS}

Researchers frequently need to analyze the behavior of a dependant variable or model output under the change of some inputs or characteristics. These intentional changes are addressed to discover a specific response on the dependant variable. Some models can have more than one dependant variable or output; this makes the experiment more complex. An experiment is defined as a test or series of tests in which changes are made to input variables in order to study the effect of them on the response dependant variable (11). The design of experiments deal with the selection of input data which can include the collection of data and definition of the changes that would be made to the data, the performance of the experiment, analysis of results, and the drawing of objective conclusions. Experimental design has become important in engineering not only in developing new design procedures but also in improving previous design procedures.

Some phenomena in engineering depend only on physical mechanisms. They can be so well understood that they can be modeled mathematically. These types of models are called mechanistic models. Other phenomena are too complex to be mathematically modeled. They require observation and experimentation to be understood in order to develop statistical models. These types of models are called empirical models. Some variables are controllable, whereas other variables are incontrollable. Some variables are easy to collect, whereas other variable are difficult and expensive to collect (11).

The objective of an experiment can include:

- Identification of the most significant variables on the response.

- Identify the set of variables that lead to a desired response value.

- Identify the set of variables that lead to a small response value.

- Identify the set of controllable variables that minimize the effect of uncontrollable variables.

Most of the experiments have several factors and that can be studied using two different approaches: one-factor-at-a-time or factorial experiment. In order to understand the differences between these approaches the concept of interaction has to be defined. Interaction is the failure 
of one factor to produce the same effect on the response at different levels of another factor. One-factor-at-a-time approach analyzes one factor at time while the other factors are fixed. This process is repeated until all factors are studied. The problem with this approach is that interactions cannot be identified. The best approach when dealing with several factors is to perform factorial experiments; in this approach several factors are varied together to analyze the interactions between factors. The variations within each factor are called levels; in general terms if an experiment has $\mathrm{k}$ factors each at 2 levels the number of runs to study all possible combination can be computed as $2^{\mathrm{k}}$. For example, 2 factors and two levels would require 4 runs, 5 factors and two levels each would require 32, 10 factors and 2 levels each would require 1024 runs. It can be seen that an experiment with many parameters may become excessively large (11).

\subsection{Basic Principles of Design of Experiments}

The statistical approach in the design of experiments is needed for drawing meaningful conclusion from the data. According to Montgomery, 2005, when the collection of data involves experimental error the statistical methods are the only objective analytical approach (11). Design of the experiment and statistical analysis of the data are the important steps to solve any experimental problem. Statistical design of experiments involved three basic principles randomization, replication, and blocking (11):

\subsubsection{Randomization:}

The collection of the data and the order in which they are processed are randomly determined. This is the basic part in the use of statistical methods in experimental design because the observations have to be independently distributed random variables.

\subsubsection{Replication}

Replication is an independent repetition of each factor combination. Replication allows estimation of the experimental error which is needed to determine if the experimental units are statistically different. Additionally, replications allow calculation of the sample mean which is used to estimate the true sample mean response. 


\subsubsection{Blocking}

A block is a set of relatively homogenous conditions. The observations are divided into groups that are run in each block. Blocking is use to reduce variability of the factors induced for extraneous factors that can influence the experimental response. This improves the precision in which the comparison of each factors are made.

\subsection{Physical Experiments and Computer Experiments}

Engineers have traditionally performed experiments in laboratory in which the experimenter tests different factors with different levels. These are physical experiments. They might obtain different outputs with the same experimental configuration due to experimental error. The analysis of the data is focused on studying the relationship between the factors and the dependant variable. A good experimental design and statistical model are required in order to draw adequate conclusion. Statistical models, like factorial experiments, are based on analysis of variance (ANOVA) and the optimum design based on regression models. These models are available for physical experiments (12).

Some engineering problems involved complex models which have been implemented into computer codes. The analysis of the problem might include mathematical models, statistical models, and complex nested procedures. These codes require several inputs and can have several outputs. The model may include the combination of different parameters that make impossible an analytical solution or the solution may include iterative calculations. The investigation of such codes has become important; especially, when the collection of the input data is expensive or requires considerable time or when running the model requires many hours of computer work. Sensitivity analysis procedures are used to deal with these types of problems. This methodology allows identification of the most sensitive parameters in complex models. Statistical sampling techniques are used to deal with the uncertainty on the response when input data are varied. Metamodels are used when the original model requires too much time to run; they are suitable approximated models, close to the real model and faster to run. A metamodel can be expressed as (12):

$$
y=g\left(x_{1}, x_{2}, \ldots x_{s}\right), \quad X \in T
$$


Where,

$\mathrm{g}$ : metamodel for the relationship between $\mathrm{y}$ and $\mathrm{x}$ (it is easy to compute and has analytical formula).

$$
\begin{aligned}
& \mathbf{X}=\text { inputs } \\
& \mathbf{T}=\text { input space } \\
& \mathrm{S}=\text { number of input parameters }
\end{aligned}
$$

A computer experiment is the repeated run of a computer code while the input data are varied according to the interest of the experimenter and acceptable input values. This type of experiment is deterministic: any time you run the code with the same data, the same output is obtained. Thus, there is not experimental error. This is why the computer experiments are different from physical experiments and different statistical techniques have been developed to design and analyze computer experiments. In computer experiments the adequacy of model fit is determined exclusively by systematic bias. The least square residual, as measurement of uncertainty, does not apply to computer experiments. The concepts of experimental units, replication, blocking, and randomization are not relevant (13).

A model to explain complicated physical phenomena is described in equation 4.2. It can be a complex mathematical model that can be analyzed in a computer experiment (12):

$$
y=f\left(x_{1}, x_{2}, \ldots x_{s}\right)=f(\boldsymbol{X}), \boldsymbol{X}=\left(x_{1}, x_{2}, \ldots x_{s}\right)^{\prime} \in \boldsymbol{T}
$$

Where,

f: Known model (complicated or has no analytical formula)

$\mathbf{X}=$ inputs

$\boldsymbol{T}$ : Input space (large in most experiments)

For a given input $\mathrm{X}$ it is possible to find the output without random error. The number of inputs is generally large in computer experiments.

\subsubsection{Sampling in Computer Experiments}

The goal of sampling in computer experiments is to spread the input data over the input space. The design developed under this concept is called Space-Filling Experimental Design (12). The statistical approach is explained by Fung, et al as follow (12): 
Consider an experimental region in a s-dimensional cube $C^{\mathrm{s}}=[0,1]^{\mathrm{s}}$. For a number of runs, $n$, the objective is to find a good design $D_{n}=\left\{x_{1}, x_{2}, \ldots x_{s}\right\}$, where $x_{i} \in C^{s}$, such that the deviation.

$$
\operatorname{Dev}(\mathbf{x} ; f ; g)=f(\mathbf{x})-g(\mathbf{x})
$$

is as small as possible, for all $\mathbf{x} \in \mathrm{C}^{\mathrm{s}}$ and $y=f(\mathbf{x})$ is the original model and $y=g(\mathbf{x})$ is the metamodel. In searching the best sampling scenario the overall mean model is used to find the best estimator of the overall mean $y$.

$$
\begin{aligned}
& E(y)=\int_{C^{S}} f(\mathbf{x}) d x \\
& \bar{y}_{\left(D_{n}\right)}=\frac{1}{n} \sum_{i=1}^{n} f\left(x_{i}\right)
\end{aligned}
$$

The sample mean method suggests using $\bar{y}_{\left(D_{n}\right)}$ as an estimator of the overall mean. The goal is to find a design $D_{n}$ such that $\bar{y}_{\left(D_{n}\right)}$ is optimal. Latin Hypercube Sampling (LHS) was introduced for estimating the optimal mean estimator $\bar{y}_{\left(D_{n}\right)}(14)$.

\subsection{Latin Hypercube Sampling}

Latin hypercube sampling (LHS) is a method for selecting input values for performing sensitive analysis on complex computer codes. The method of choosing the sample ensures that all areas of the input space are represented by the input values and the input values are evenly spread out over the input space of each parameter (15). Latin Hypercube Design (LHD) experiments are based on LHS. To introduce the concept of LHD suppose an experimental region equal to the unit square $[0,1]^{2}$. To generate a sample size $n$, divide each axis $[0,1]$ into $n$ equally space intervals. This procedure divides the experimental region $[0,1]^{2}$ into $n^{2}$ cells of equal size. Fill the cells with integers $1,2,3, \ldots \mathrm{n}$ in such a way that every integer should appear only once in every row and column of the grid of cells. Select one of these integers at random and randomly select a point from each cell containing this integer. The resulting $\mathrm{n}$ points are a LHS of size $n(14)$.

The procedure to obtain a LHS is explained by Santner et.al. as follows (14): a LHS of size $\mathrm{n}$ from $\mathbf{X}=\left(\mathrm{X}_{1}, \mathrm{X}_{2}, \mathrm{X}_{3} \ldots, \mathrm{X}_{\mathrm{d}}\right)$ with $\mathbf{X}$ having independently distributed variables. The range of each variable is divided into $\mathrm{n}$ intervals of equal probability as in figure 4.1 (15). The group of 
all possible Cartesian products of these intervals constitutes a division of the d-dimensional sample space into $n^{d}$ cells. A set of the $n$ cells is chosen from the $n^{d}$ population of cells in such a way that the projections of the center of the cells onto each axis are uniformly spread across the axis and then a point is chosen at random from each from each selected cell.

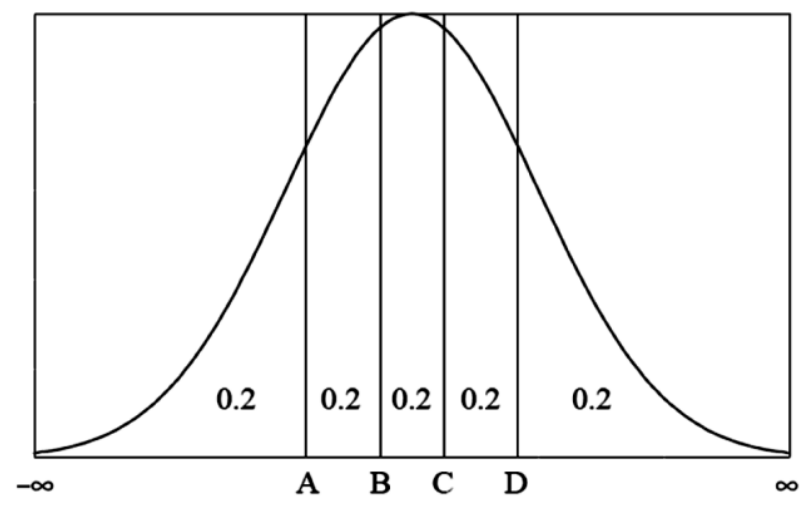

FIGURE 4.1 Division of the Density Function of a Variable (Matala, 2008)

\subsubsection{Latin Hypercube Sampling Algorithm}

A LHS with $\mathrm{n}$ runs and $\mathrm{d}$ input variables, $\operatorname{LHS}(\mathrm{n}, \mathrm{d})$ is a $\mathrm{n} \times \mathrm{d}$ matrix in which each column is a random permutation of $n=(1,2,3, \ldots, n)$. The LHS can be generated from the following algorithm which has two steps (12).

\section{- Step 1:}

Generate a LHD (n, d) as follows. Independently take d permutations of $\pi_{j}(1), \pi_{j}(2), \ldots, \pi_{j}(n)$ of the integers $1,2, \ldots, n$ for $j=1,2, \ldots, d$.

\section{- Step 2:}

Take $\mathrm{n} x \mathrm{~d}$ uniform variates (random numbers between 0 and 1 ), $U_{k}^{j} \sim U(0,1), \mathrm{k}=1,2, \ldots, \mathrm{n}$, and $\mathrm{j}=1,2, \ldots, \mathrm{d}$, which are mutually independent.

Let $\boldsymbol{X}_{k}=\left(x_{k}^{1}, x_{k}^{2} \ldots, x_{k}^{d}\right)$ where,

$$
x_{k}^{j}=\frac{\pi_{j}(k)-U_{k}^{j}}{n}, k=1,2, \ldots, n ; j=1,2, \ldots, d .
$$

Then $D_{n}=\left\{\boldsymbol{X}_{1}, \boldsymbol{X}_{2}, \ldots, \boldsymbol{X}_{n}\right\}$ is a LHS (n, d) 
The procedure for sampling can be written as (16).

- Divide the input variable ranges into $n$ equally probable intervals.

- Generate a sequence of $\mathrm{n}$ uniform random $\mathrm{U}[0,1]$ values and calculate $x_{k}^{j}$.

- Generate a random order for each sample of $\boldsymbol{X}_{k}$ that is a random ordered vector generated for each sample.

- Combine the permutated samples into $\mathrm{n}$ input vectors by matching corresponding values.

$$
\boldsymbol{X}_{j}=\left(X_{1[j]}, X_{2[j]}, \ldots X_{k[j]}\right) \quad j=1,2,3 \ldots n
$$

- Use the computer model to calculate the response $Y_{j}$ for each vector $\boldsymbol{X}_{1}, \boldsymbol{X}_{2}, \ldots \boldsymbol{X}_{n}$.

\subsection{Sensitivity Analysis Based on Regression Analysis}

The primary purpose of the sensitivity analysis is to rank the importance of the input variables. This quantifies how much the output is dependent on each specific input variable. This can be addressed by regression analysis and the inspection of the regression coefficients. The input variables selected are used to run the models and the outputs are fitted with a regression analysis model. The objective of this regression is not to predict output values but to compare the input effects on the output.

The general form of a multiple regression model can be written as:

$$
y_{i}=\propto+\beta_{1} x_{i 1}+\cdots+\beta_{s} x_{i s}+\varepsilon_{i}=\propto+\sum_{\mathrm{j}=0}^{\mathrm{s}} x_{\mathrm{ij} \beta_{\mathrm{j}}}+\varepsilon_{i}
$$

Where $\varepsilon_{\mathrm{i}}$ is the random error and $\propto, \beta_{1}, \ldots, \beta_{\mathrm{s}}$ are regression coefficients.

The regression coefficients $\beta_{s}$ are a direct indicator of sensitivity of the parameters, $x_{i}$. The more reasonable procedure to compare the regression coefficients is done by standardizing all the variables as follows:

$$
\frac{y_{i}-\bar{y}}{s_{y}}, \frac{x_{i j}-\bar{x}}{s_{j}}, j=1, \ldots s
$$


Where $\bar{y}$ and $s_{y}$ is the sample mean and sample standard deviation of y, and $\bar{x}_{j}$ and $s_{j}$ are the sample mean and sample standard deviation of $x_{i j}$. The regression model is fitted to the

standardized data and its regression coefficients are given by $\frac{b_{j} s_{j}}{s_{y}}, j=1, \ldots s$. These coefficients are called standardized regression coefficients (SRC) and the output is most sensitive to those inputs whose standardized regression coefficient is larger in absolute value because all variables have been transferred to the same scale (12).

\subsection{Method of Least Squares}

The method of least squares accounts for the deviation of each observation from its expected values and finds the coefficients $\beta_{s}$ by minimizing the sum of squared deviations (12).

$$
S\left(\beta_{0,} \beta_{1}, \ldots, \beta_{s}\right)=\sum_{i=1}^{n}\left(y_{i}-\sum_{j=0}^{S} x_{i j} \beta_{j}\right)^{2}
$$

The matrix notation of the least squares solution is given by.

$$
\boldsymbol{y}=\left(\begin{array}{c}
y_{1} \\
\vdots \\
y_{n}
\end{array}\right), \boldsymbol{X}=\left(\begin{array}{ccc}
x_{10} & \ldots & x_{1 s} \\
\vdots & \ldots & \vdots \\
x_{n 0} & \ldots & x_{n s}
\end{array}\right), \boldsymbol{\beta}=\left(\begin{array}{c}
\beta_{0} \\
\vdots \\
\beta_{s}
\end{array}\right), \boldsymbol{\varepsilon}=\left(\begin{array}{c}
\varepsilon_{1} \\
\vdots \\
\varepsilon_{n}
\end{array}\right)
$$

Equation 4.8 can be written in the matrix form.

$$
y=X \beta+\varepsilon
$$

$\mathbf{X}$ is known as the design matrix. Equation 4.10 can be written as.

$$
\boldsymbol{S}(\boldsymbol{\beta})=(\boldsymbol{y}-\boldsymbol{X} \boldsymbol{\beta})^{\prime}(\boldsymbol{y}-\boldsymbol{X} \boldsymbol{\beta})
$$

The normal equations are found by differentiating $\boldsymbol{S}(\boldsymbol{\beta})$ with respect to $\beta$,

$$
\boldsymbol{X}^{\prime} \boldsymbol{y}=\boldsymbol{X}^{\prime} \boldsymbol{X} \boldsymbol{\beta}
$$

By the inversion of $\boldsymbol{X}^{\prime} \boldsymbol{X}$ the least squares estimators of $\beta$ are defined.

$$
\widehat{\boldsymbol{\beta}}=\left(X^{\prime} X\right)^{-1} X^{\prime} \boldsymbol{y}
$$




\subsection{Hypothesis Testing in Regression Analysis}

Hypothesis testing in linear multiple regression analysis addresses the usefulness of the regression model and the relationship among the dependent variable with the independent variables. Several statements about the regression coefficients may be tested in hypothesis testing. Test for significance of the regression model and test for the significance of individual coefficients are explained in this section.

\subsubsection{Test for the Significance of the Regression}

The test for significance of the regression evaluates the linear relationship among the dependant variable and the independent variables. The null hypothesis $\left(\mathrm{H}_{0}\right)$ and alternative hypothesis $\left(\mathrm{H}_{1}\right)$ are explained as follows (11).

$$
\begin{gathered}
H_{0}: \beta_{1}=\beta_{2}=\cdots=\beta_{k}=0 \\
H_{1}: \beta_{j} \neq 0 \text { for at least one } j
\end{gathered}
$$

Where,

$\beta_{\mathrm{i}}:$ Regression coefficients

If $\mathrm{H}_{0}$ if is not rejected, the data provide evidence that all regression coefficients are zero. In others words, there is a not linear relationship among dependant and independent variables. If $\mathrm{H}_{0}$ is rejected, at least one of the independent variables in the model explains the variation of the dependant variable. The $\mathrm{F}$ test is used to test the significance of $\mathrm{H}_{0}$. The statistics is computed by equation 4.16 (11).

$$
F_{0}=\frac{M S_{R}}{M S_{E}}
$$

Where,

$\mathrm{MS}_{\mathrm{R}}$ : Sum of squares due to the model

$\mathrm{MS}_{\mathrm{E}}$ : Sum of squares due to the residual (error)

$\mathrm{H}_{0}$ is rejected if $\mathrm{F}_{0}$ is larger than $\mathrm{F}_{\alpha, \mathrm{k}, \mathrm{n}-\mathrm{k}-1}$ from the $\mathrm{F}$ distribution. Where $\alpha$ is the significance level, $\mathrm{k}$ is the number of independent variables, $\mathrm{n}$ degrees of freedom in the numerator, and $\mathrm{n}-\mathrm{k}-1$ 
are the degrees of freedom in the denominator. Usually, P-value is used for hypothesis testing. Pvalue is the smallest level of significance for which $\mathrm{H}_{0}$ would be rejected (11). Then, if the P-value for $F_{0}$ is smaller than the level of significance $\alpha, H_{0}$ is rejected.

\subsubsection{Test for Individual Regression Coefficients}

This test evaluates whether the effect of an independent variable is significance to explain the variation of the dependent variable in the multiple regression model. The null and alternative hypotheses are indicated as follow (11).

$$
\begin{aligned}
& H_{0}: \beta_{j}=0 \\
& H_{1}: \beta_{j} \neq 0
\end{aligned}
$$

If the $\mathrm{H}_{0}$ is not rejected, then the independent variable associated with that regression coefficient is not significant in explaining the variability of the dependant variable in the model. The $t$ test is used to test the significance of $\mathrm{H}_{0}$. The statistics is computed from equation 4.17 (11).

$$
t_{0}=\frac{\widehat{\beta}_{j}}{\sqrt{\widehat{\sigma}^{2} C_{j j}}}
$$

Where,

$\beta_{\mathrm{i}}:$ Regression coefficients

$\mathrm{Cjj}$ : Diagonal element of $\left(\mathrm{X}^{\prime} \mathrm{X}\right)-1$ corresponding to the $\widehat{\beta}_{\mathrm{j}}$

$\sqrt{\widehat{\sigma}^{2} C_{j j}}$ : Standard error of the regression coefficient $\widehat{\beta}_{j}$. It usually is expressed as $\operatorname{se}\left(\widehat{\beta}_{\mathrm{j}}\right)$ therefore equation 4.17 becomes:

$$
t_{0}=\frac{\widehat{\beta}_{j}}{s e\left(\widehat{\beta}_{\mathrm{j}}\right)}
$$

As in the test for significance of the regression, the P-value approach is also frequently used to test individual regression coefficients. Then, if the P-value for $\mathrm{t}_{0}$ is smaller than the level of significance $\alpha, \mathrm{H}_{0}$ is rejected. 


\subsection{Rank Transformation}

Rank transformation has been successfully used in sensitivity analysis based on regression analysis when there is a poor regression fit or there is evidence of collinearity or nonlinearity among independent variables (17). It also reduces the effects of extreme values (18). Rank transformation is a robust statistical technique to replace the data for their rank (12). Both independent and dependant variables are rank transformed as follows (14). For every independent and dependant variable, the smallest value is replaced by 1 , the next by 2 , and so on until the highest term is replaced by $\mathrm{n}$ where $\mathrm{n}$ is the number of values. Then standard regression analysis is performed on the transformed data. Generally, rank transformation has better regression fit than the original data.

\subsection{Sensitivity Based on Gaussian Stochastic Models}

The following discussion of using stochastic Gaussian models for sensitivity analysis is based on reference (19). The sensitivity of complex models can be analyzed by fitting the input and outputs from the model to a Gaussian stochastic model. The inputs are defined as $\mathbf{x}_{\mathrm{i}}$ with $\mathrm{i}=$ $1,2,3, \ldots \mathrm{n}$, and the outputs are defined as $\mathbf{y}_{\mathrm{i}}$ with $\mathrm{i}=1,2,3 \ldots \mathrm{n}$, where $\mathrm{n}$ is the number of runs. The stochastic model is described in equation 4.19 (19).

$$
y(x)=\mu+Z(x)
$$

Where, $\boldsymbol{\mu}$ is the mean of $\boldsymbol{y}(\boldsymbol{x})$ and $\mathbf{Z}(\mathbf{x})$ is a random process that is assumed to have mean zero and covariance between two input vectors $\mathbf{x}, \mathbf{x}^{\prime}$ given by,

$$
\operatorname{Cov}\left(\boldsymbol{Z}(\boldsymbol{x}), \boldsymbol{Z}\left(\boldsymbol{x}^{\prime}\right)\right)=\boldsymbol{\sigma}^{2} \boldsymbol{R}\left(\boldsymbol{x}, \boldsymbol{x}^{\prime}\right)
$$

Where, $\sigma^{2}$ is the variance of the stochastic process, $\mathrm{Z}(\mathrm{x})$, and $\mathrm{R}\left(\mathrm{x}, \mathrm{x}^{\prime}\right)$ is a correlation function that is estimated from the design data or input and output values.

The main idea of this procedure is that two outputs are likely to be similar when their input vectors are close assuming continuity and smoothness. As the distance between the two input vectors decreases, the similarities of their outputs is likely to increase, and vice versa (19). 
A general approach is assumed the stochastic process, $\mathrm{Z}(\mathrm{x})$, to be Gaussian and defined by the products $(20)$.

$$
\boldsymbol{R}\left(\boldsymbol{x}, \boldsymbol{x}^{\prime}\right)=\prod_{j=1}^{K} \exp \left(-\theta_{j}\left|\boldsymbol{x}_{j}-\boldsymbol{x}_{j}^{\prime}\right|^{p_{j}}\right)
$$

Where, $\theta_{\mathrm{j}} \geq 0$ and $0<\mathrm{p}_{\mathrm{j}} \leq 2$ for $\mathrm{p}=1$ the product $\mathrm{R}\left(\mathrm{x}, \mathrm{x}^{\prime}\right)$ is a linear correlation function, $\mathrm{p}$ $=2$ gives differentiable correlation functions $\mathrm{k}$ is the number of input parameters, and $\theta_{\mathrm{j}}$ control the variability of the response, $\theta_{\mathrm{j}}$ increases when the variation is more local (20). The parameter $\theta_{\mathrm{j}}$ is calculated by numerical estimation of the maximum likelihood that is function of the correlation parameter and the output data. Several algorithms have been developed to perform the computer iteration to compute $\theta_{\mathrm{j}}(19)$.

Equation 4.21 respects the deterministic nature of the computer codes by giving $\mathrm{R}(\mathrm{x}, \mathrm{x})=1$. So, when input vector replications occur their prediction are equal (19).

The predictions of the output from the computer code are based on the best linear unbiased predictor (BLUP) of $\mathbf{y}$ for an untried $\mathbf{x}$ as indicated in equation 4.22 (19).

$$
\hat{y}=\hat{\mu}+\boldsymbol{r}^{T}(\boldsymbol{x}) \boldsymbol{R}^{-\mathbf{1}}(\boldsymbol{y}-\mathbf{1} \hat{\mu})
$$

Where,

$-\boldsymbol{r}^{T}(\boldsymbol{x})=\mathrm{n} \times 1$ vector of correlations between $\mathbf{x}$ and of each of the $\mathrm{n}$ design points with element i given by $\boldsymbol{R}\left(\boldsymbol{x}, \boldsymbol{x}_{\boldsymbol{i}}\right)$.

- $\quad \boldsymbol{R}=n \times n$ correlation matrix with element $\left(i, i^{\prime}\right)$ given by $\boldsymbol{R}\left(\boldsymbol{x}, \boldsymbol{x}_{i}^{\prime}\right)$.

$-\hat{\mu}=\frac{\mathbf{1}^{T} \boldsymbol{R}^{-1} \boldsymbol{y}}{\mathbf{1}^{T} \boldsymbol{R}^{-1} \mathbf{1}}$ is the generalized least-square estimator of $\mu$.

The main effects of the factor can be computed as follows (13),

$$
\hat{\mu}_{i}\left(x_{i}\right)=\int y(x) \prod_{j \neq i} d x_{i}-\hat{\mu}_{0}
$$

Where $\hat{\mu}_{0}$ is the average response computed by,

$$
\hat{\mu}_{0}=\int y(x) \prod_{i \neq 1} d x_{i}
$$




\section{CHAPTER 5: RESEARCH METHODOLOGY}

This research focuses on the determination of the sensitivity of flexible pavement designs using the Mechanistic-Empirical Pavement Design Guide input parameters. The sensitivity analysis was based on computer experiments using a statistical basis to design the experiment. This was accomplished by a sensitivity analysis of the performance parameter in the MEPDG. Latin Hypercube Sampling was used to perform a space-filling sampling over the entire space for all parameters in study and multiple regression analysis was used to build metamodels to analyze the relative importance of parameters in the models using the standardized coefficients technique. Figure 5.1 displays the approach for the computer experiment.

\subsection{Research Questions and Hypothesis}

The complexity of the models, the large numbers of input parameters, the hierarchical characteristic of the inputs, and the need of calibration to local condition are the basis of the primary research questions:

- What parameters are most sensitive on the performance response of the pavements?

- What is the most efficient investment for characterizing the input parameters with limited resources?

- What parameters may have hierarchical level 1,2 or 3 without affecting in considerable manner the predicted pavement response?

- Is this methodology appropriate to identify sensitive parameters in the design procedure?

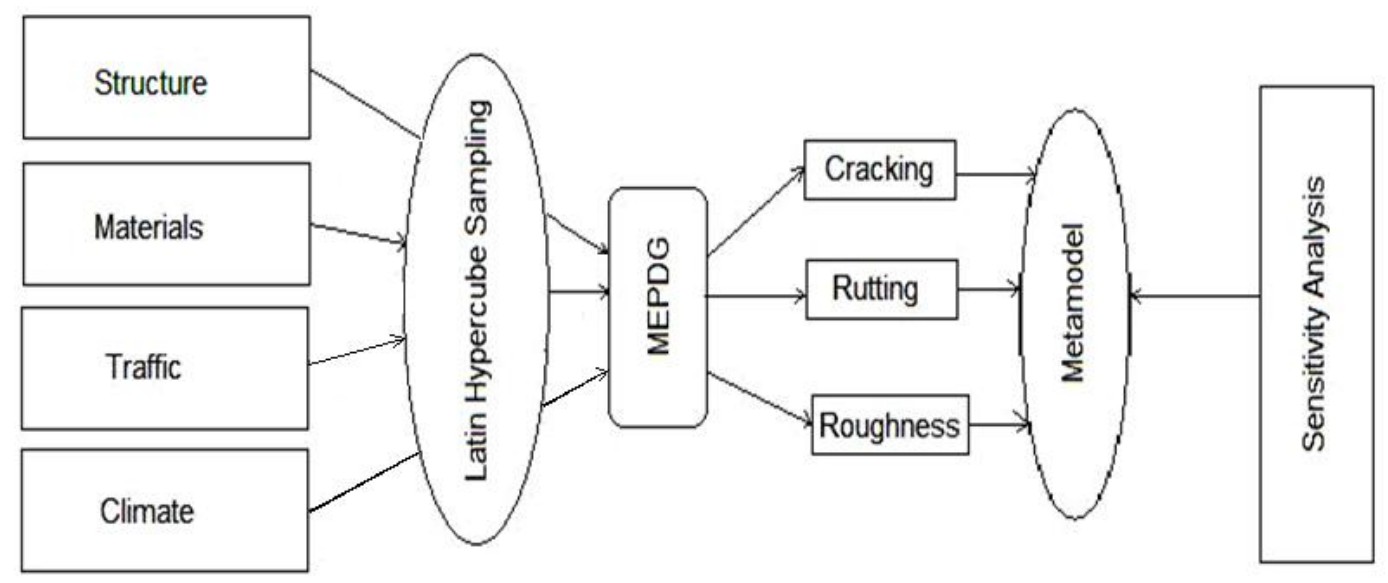

FIGURE 5.1 Computer Experiment Approach 


\subsection{Research Design and Methodology}

The computer experiments analyzed two typical flexible pavement structures used in West Virginia. The project site information was fixed to Morgantown, WV, which set the climatic condition. The experimental matrix is displayed in Table 5.1. Two analyses were evaluated, material and traffic. The experiment analyzed the sensitivity of material properties and traffic loads separately. In the first step, the sensitivity of traffic inputs on performance were analyzed for the two structures. The computer experiment was run with random sampling over the entire input parameter spaces of traffic having the material properties unchanged for each pavement structure. In the second step, the sensitivity of the material properties on performance for each structure was analyzed for a fixed traffic condition. The computer experiment was run with random sampling over the entire input parameter space of material properties having traffic parameters unchanged.

The information gathered in the previous steps was analyzed using the sensitivity analysis techniques explain previously, regression analyses with standardized coefficients. The outputs of interest were cracking, rutting, and roughness at the end of the simulated design period.

TABLE 5.1 Experimental Matrix

\begin{tabular}{|c|c|c|}
\hline & \multicolumn{2}{|c|}{ STRUCTURE (S) } \\
\hline & $\mathrm{S}_{1}$ & $\mathrm{~S}_{2}$ \\
\hline $\begin{array}{l}\text { Sensitivity to Traffic (T) } \\
\text { (Fixed materials) }\end{array}$ & $\mathrm{TSS}_{1}$ & $\mathrm{TSS}_{2}$ \\
\hline $\begin{array}{l}\text { Sensitivity to Material (M) } \\
\text { (Fixed traffic) }\end{array}$ & $\mathrm{MSS}_{1}$ & $\mathrm{MSS}_{2}$ \\
\hline
\end{tabular}

The general steps for the research were:

1. Perform a thorough review of the input parameters for the MEPDG.

2. Select the parameter used in the sensitivity analysis. This step included the definition of fixed and varied parameters.

3. Define the ranges of variation for each parameter included in the sensitivity analysis. This evaluation considered the correlation and default values recommended in the MEPDG and local data for level 3. (Appendix A). 
4. The sample size (n) for the computer experiment was defined as 10 times the number of input parameters in the sensitivity analysis. This sample size defines the number of runs for each factor combination in the computer experiment.

5. The Latin Hypercube Sampling for every factor combination was performed over the entire space of the input parameters considered in the sensitivity analysis.

6. Model the output using multiple regression analysis (metamodel). The dependant variables selected for this analysis were cracking, rutting, and roughness at the end of the design period.

7. Perform the sensitivity analysis of the input parameters by analyzing the standardized regression coefficients.

8. Analysis and evaluation of results.

An example of the matrix of results is displayed on Table 5.2. The table presents the material properties and their sensitivity to rutting, cracking, and IRI. The sensitivity is indicated by the sign of the SRC of significant parameters. A positive sign (+) indicates that the parameter is significant with a positive SRC. A negative sign (-) indicates that the parameter is significant with a negative SRC. Letter $\mathrm{n}$ indicates that the parameter is not significant. A positive SRC indicates that the corresponding MEPDG output increases as the input value for the parameter increases. A negative SRC indicates that the corresponding MEPDG output decreases as the input value for the parameter increases.

TABLE 5.2 Matrix of Results Layout

\begin{tabular}{|c|c|c|c|}
\hline \multirow{2}{*}{ PARAMETER } & \multicolumn{3}{|c|}{ Sign of SRC } \\
\cline { 2 - 4 } & RUTTING & CRACKING & IRI \\
\hline Mr & + & + & + \\
\hline $\mathrm{E}$ & + & - & - \\
\hline$\mu$ & + & $\mathrm{n}$ & + \\
\hline. & - & $\mathrm{n}$ & $\mathrm{n}$ \\
\hline. & + & - & - \\
\hline. & $\mathrm{n}$ & $\mathrm{n}$ & $\mathrm{n}$ \\
\hline
\end{tabular}




\subsubsection{Pavement Structures}

Two structures were considered in the analysis.

- Structure 1 is a full-depth asphalt concrete similar to designs used in the state of West Virginia (figure 5.2).

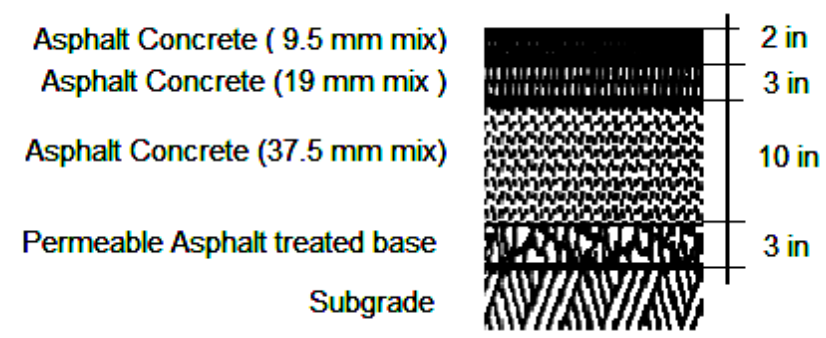

FIGURE 5.2 Pavement Structure 1

- Structure 2 includes conventional asphalt pavement structure (figure 5.3).

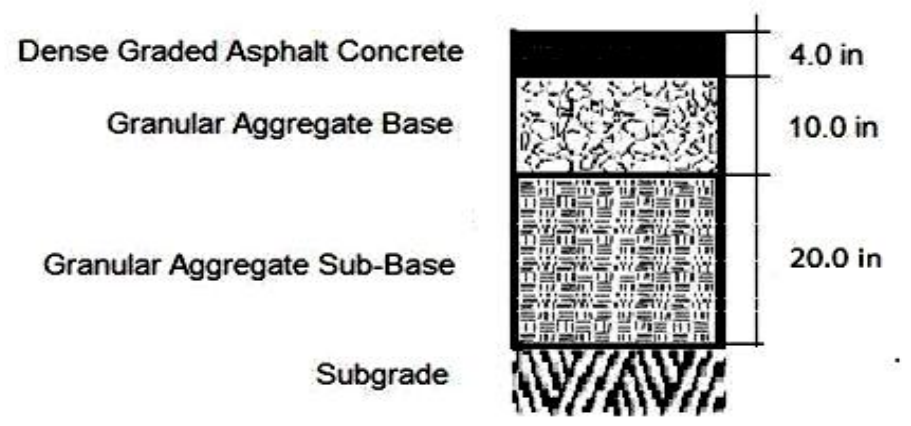

FIGURE 5.3 Pavement Structure 2

\subsubsection{Project Information}

The project location was set to Morgantown, WV. So, the project information is associated with this location. The general information inputs for a project are:

\section{- General Information}

- Design Life

- Base/Subgrade construction month

- Pavement construction date

- Date open to traffic 
- Type of pavement (flexible)

- Location

- Traffic direction

\subsubsection{Input Values and Ranges in the Experiment}

The input values and ranges for structures 1 and 2 are presented in Appendix A. The tables in the appendix show the parameters; inputs values are given for fixed parameters and ranges are given for the variable parameters. The total numbers of input parameters required for each structure are displayed in Table 5.3.

TABLE 5.3 Total Numbers of Input Parameters

\begin{tabular}{|l|c|c|}
\hline \multicolumn{1}{|c|}{ INPUT TYPE } & Structure 1 & Structure 2 \\
\hline General Information & 5 & 5 \\
\hline Site project identification & 5 & 5 \\
\hline Analysis parameters & 7 & 7 \\
\hline Traffic & 26 & 26 \\
\hline Climate & 5 & 5 \\
\hline Structure & 92 & 105 \\
\hline \multicolumn{1}{|c|}{ Total } & $\mathbf{1 4 0}$ & $\mathbf{1 5 3}$ \\
\hline
\end{tabular}

\subsubsection{Analysis process}

The outputs from the computer experiment selected for this analysis were cracking, rutting, and roughness at the end of the design period. The inputs and outputs of the computer experiment were standardized according to equation 4.9. Then, the standardized inputs and outputs were fitted to multiple regression models to find the standardized regression coefficients (SRC). The value of the standardized regression coefficients are indicators of the importance of parameters. Standardized coefficients were used to classify the relative importance of input parameters regarding to cracking, rutting, and roughness. 


\section{CHAPTER 6: SENSITIVITY OF MEPDG TO TRAFFIC PARAMETERS}

This chapter presents the sensitivity analyses of MEPDG to traffic parameters. The analysis was performed on structures 1 and 2, Figures 5.2 and 5.3. The steps used in this study were:

1. Analysis of traffic inputs to choose the parameters for the analysis (specified in appendix A).

2. Determine the input space for each parameter (ranges of input values).

3. Latin Hypercube Sampling (LHS) of the entire input space of all parameters in the analysis.

4. Run MEPDG for each input set selected with LHS.

5. Outputs from MEPDG were analyzed using multiple regressions with standardized coefficient in order to categorize the relative importance among input parameters.

The MEPDG outputs evaluated during this study were IRI, rutting, and cracking.

\subsection{Traffic Parameters in the Analysis}

Input parameters and their ranges used in the study are shown in Table 6.1.

TABLE 6.1 Input Parameters and Ranges

\begin{tabular}{|l|c|}
\hline \multicolumn{1}{|c|}{ INPUT PARAMETER } & RANGE \\
\hline Two-way AADT (Structure 1) & $10000-40000$ \\
\hline Two-way AADT (Structure 2) & $1000-4000$ \\
\hline Percent of heavy vehicles & $40-60$ \\
\hline Percent of trucks in design direction & $40-60$ \\
\hline Percent of trucks in the design lane & $70-95$ \\
\hline Vehicle operational speed & $40-70$ \\
\hline Traffic growth factor (compound) & $1-8$ \\
\hline Mean wheel location & $5-36$ \\
\hline Traffic wander standard deviation & $7-13$ \\
\hline Average axle width & $8-10$ \\
\hline Dual tire spacing & $5-24$ \\
\hline
\end{tabular}

The regression analysis approach was used to evaluate the sensitivity of MEPDG distress predictions to various input parameters. A restriction of regression analysis is the input parameters in the analysis should not have collinearity. As shown in equation 4.15, the 
regression coefficients are computed from $\widehat{\boldsymbol{\beta}}=\left(\boldsymbol{X}^{\prime} \boldsymbol{X}\right)^{-\mathbf{1}} \boldsymbol{X}^{\prime} \boldsymbol{y}$. Collinearity may result in singularity of $\boldsymbol{X}^{\prime} \boldsymbol{X}$, which in such case has no inverse so regression coefficients cannot be computed (22). Il-collinearity or near collinarity, meaning near dependence, is also undesirable because it leads to unreliable estimates of the regression coefficients (22).

\subsection{Latin Hypercube Sampling for Traffic Sensitivity Analysis}

A LHS was performed over the input parameters and ranges in Table 6.1. The number of runs was defined as 10 times the number of parameter. A total of 100 runs were defined. Figure 6.1 shows a part of the LHS and the output from MEPDG of IRI, rutting, and cracking. The entire LHS is presented in appendix B.

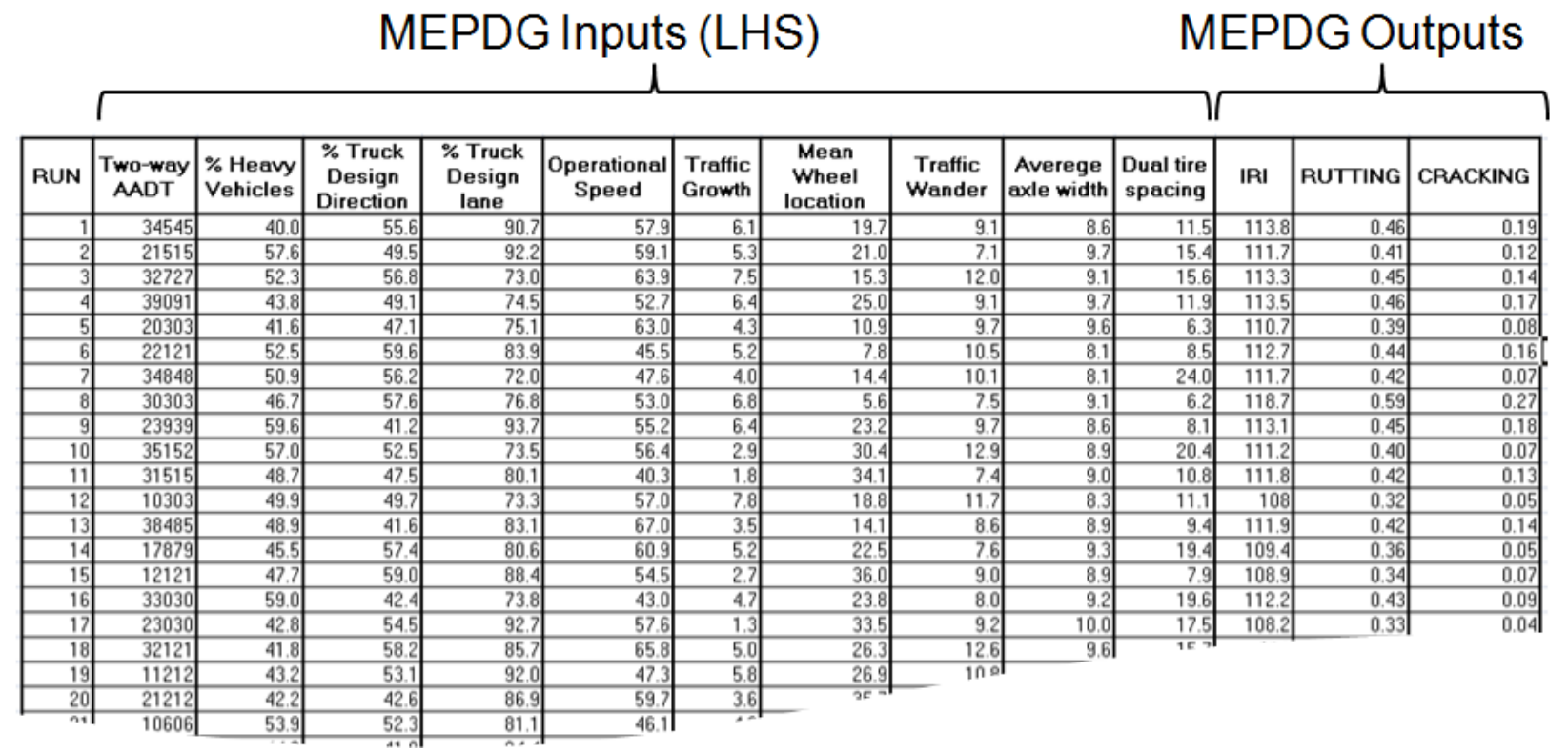

FIGURE 6.1 Latin Hypercube Sampling and MEPDG Output

A multivariate analysis was performed to evaluate the relationship between individual pairs of input parameters in the LHS. Table 6.2 displays the pairwise correlation coefficients for each pair of input parameters. Each row and each column correspond to an input parameter. Notice that all pairwise correlations have small values. For example, the correlation between two-way AADTT and traffic growth is -0.0251 . No relationships among individual parameters were found in the LHS. Since the values for the input parameters are generated in an independent and random manner, one would expect independence of the input parameters. 
TABLE 6.2 Pairwise Correlations Coefficients Among Input Parameters

\begin{tabular}{|c|c|c|c|c|c|c|c|c|c|c|}
\hline & $\begin{array}{c}\text { Two-way } \\
\text { AADT }\end{array}$ & $\begin{array}{l}\text { \%Heavy } \\
\text { Vehicles }\end{array}$ & $\begin{array}{c}\text { \% Truck } \\
\text { Design } \\
\text { Direction }\end{array}$ & $\begin{array}{c}\text { \% Truck } \\
\text { Design } \\
\text { Lane }\end{array}$ & $\begin{array}{c}\text { Operational } \\
\text { Speed }\end{array}$ & $\begin{array}{l}\text { Tracffic } \\
\text { Growth }\end{array}$ & $\begin{array}{c}\text { Mean } \\
\text { Wheel } \\
\text { Location }\end{array}$ & $\begin{array}{c}\text { Traffic } \\
\text { Wander }\end{array}$ & \begin{tabular}{|c|} 
Average \\
Axle width
\end{tabular} & $\begin{array}{c}\text { Dual Tire } \\
\text { Spacing }\end{array}$ \\
\hline Two-way AADT & 1 & 0.0389 & -0.0541 & -0.0238 & 0.0082 & -0.0251 & 0.0314 & -0.051 & -0.0303 & 0.0279 \\
\hline$\%$ Heavy Vehicles & 0.0389 & 1 & -0.0237 & -0.0247 & 0.0133 & -0.0207 & 0.0333 & -0.0665 & 0.0036 & -0.0202 \\
\hline \% Truck Design Direction & -0.0541 & -0.0237 & 1 & -0.0394 & -0.0439 & -0.04 & 0.0043 & 0.0095 & 0.0674 & 0.0372 \\
\hline \% Truck Design Lane & -0.0238 & -0.0247 & -0.0394 & 1 & 0.074 & -0.0174 & 0.0801 & 0.0318 & 0.0482 & -0.0015 \\
\hline Operational Speed & 0.0082 & 0.0133 & -0.0439 & 0.074 & 1 & -0.0533 & -0.0083 & 0.0501 & -0.0168 & -0.0213 \\
\hline Tracffic Growth & -0.0251 & -0.0207 & -0.04 & -0.0174 & -0.0533 & 1 & -0.1539 & 0.0493 & -0.0707 & -0.022 \\
\hline Mean Wheel Location & 0.0314 & 0.0333 & 0.0043 & 0.0801 & -0.0083 & -0.1539 & 1 & 0.0316 & 0.1363 & 0.0224 \\
\hline Traffic Wander & -0.051 & -0.0665 & 0.0095 & 0.0318 & 0.0501 & 0.0493 & 0.0316 & 1 & 0.023 & -0.0502 \\
\hline Average Axle width & -0.0303 & 0.0036 & 0.0674 & 0.0482 & -0.0168 & -0.0707 & 0.1363 & 0.023 & 1 & -0.0475 \\
\hline Dual Tire Spacing & 0.0279 & -0.0202 & 0.0372 & -0.0015 & -0.0213 & -0.022 & 0.0224 & -0.0502 & -0.0475 & 1 \\
\hline
\end{tabular}

Figure 6.2 shows pair comparisons of the spread of the sampled values. It is important to notice that the projections of the points are spread out over the axis and no trends, linear or nonlinear, are observed in the figure. These are desired characteristic in this study.

The rows of the LHS sample in figure 6.1 were used as inputs to run MEPDG. The sensitivity of input parameters over IRI, rutting, and cracking were analyzed using multiple regression analysis with standardized coefficients. This analysis was done for both structures 1 and 2.

The sensitivity analysis was performed on structure 1 and 2, Figure 5.2 and 5.3. The 100 runs defined in the LHS were input in MEPDG for each pavement structure and run using batch mode. Two batches of 50 runs were performed for each structure. Using a computer with a core i7 processor, $2.8 \mathrm{GHz}$, and $4 \mathrm{~GB}$ RAM in a 32-bit operating system, the average running time was 15 minutes for a single run. 12.5 hours of continuous computer processing were required for each batch, a total of 25 hours of computer work for each pavement structure. 


|

FIGURE 6.2 Pairwise Correlations Among Input Parameters

\subsection{Results of Traffic Sensitivity of Structure 1}

\subsubsection{IRI Sensitivity to Traffic Input Parameters on Structure 1}

A multiple regression model with the input parameters in the LHS and IRI was performed. The regression coefficients (RC) are indicators of the importance of the parameters, and the magnitude of the standardized regression coefficient (SRC) is an indicator of the relative importance among input parameters. A good regression model means that much of the variation of the model output in response to its inputs is explained by the regression model. 


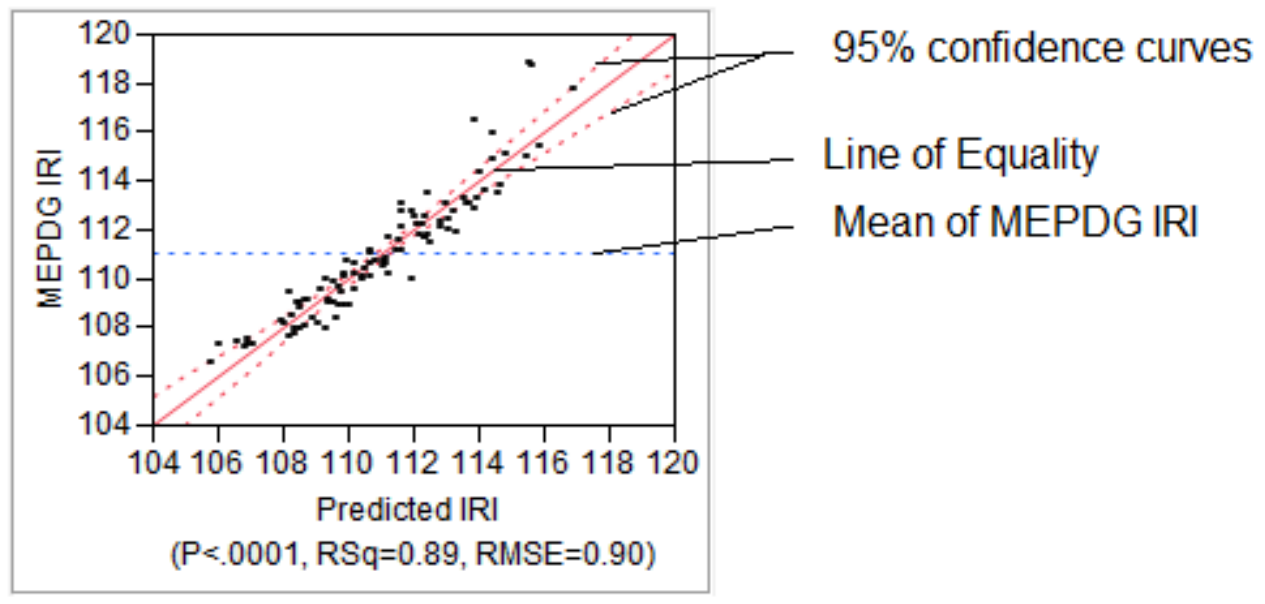

FIGURE 6.3 IRI Actual by Predicted Plot for Structure 1

Figure 6.3 compares the IRI output from MEPDG to the predicted IRI from the regression model. The line of equality (LOE) shows where the output from MEPDG and the predicted IRI are equal. The vertical distance from a point to the LOE is the difference between MEPDG output and the predicted IRI from the regression model. The horizontal dashed line represents the MEPDG IRI mean. The distance from a point to the mean line represents the residual without any effect in the model. The two dashed lines represent a $95 \%$ confidence interval. The P-value is smaller than 0.0001 which indicates that the regression model is significant. This result is confirmed by the multiple regression coefficient, $\mathrm{R}^{2}=0.89$. Root Mean Square Error, RMSE, is an estimator of the standard deviation. RMSE $=0.9013$ is small compared to the magnitude of IRI values.

Table 6.3 shows the IRI regression coefficients and their standard errors. The t-ratios are computed by dividing each coefficient by its standard error as indicated in equation 4.18. The bar graph shows the t-ratios with vertical lines showing the 0.05 significance level. The input parameters are sorted from highest to lowest according to the absolute value of the t-ratios. Some regression coefficients have a positive value indicating that as the parameters increase the IRI increases. Other parameters have negative regression coefficients indicating that as the parameters increase IRI decreases. Increasing dual tire spacing, traffic wander, and operational speed would lead to a lower IRI output from MEPDG. The contrary is also true for the other parameters in the table. Although these variables are beyond the control of pavement designers, the significance demonstrates they should be careful in estimating these values for design. 
TABLE 6.3 IRI Regression Coefficients for Structure 1

Term
\begin{tabular}{|l||r|r|r|}
\hline Two-way AADT & Coefficient & \multicolumn{1}{c|}{ Std Error } & \multicolumn{1}{c|}{ Ratio } \\
Tracfic Growth & 3.019 & 0.155 & 19.43 \\
Dual tire spacing & 1.834 & 0.157 & 11.66 \\
\% Truck Design Direction & -1.714 & 0.155 & -11.04 \\
\% Heavy Vehicles & 0.981 & 0.156 & 6.30 \\
Traffic Wander & 0.802 & 0.155 & 5.16 \\
\% Truck Design lane & -0.768 & 0.156 & -4.93 \\
Operational Speed & 0.723 & 0.156 & 4.64 \\
Average axle width & -0.382 & 0.156 & -2.45 \\
Mean Wheel location & 0.044 & 0.157 & 0.28 \\
\hline
\end{tabular}

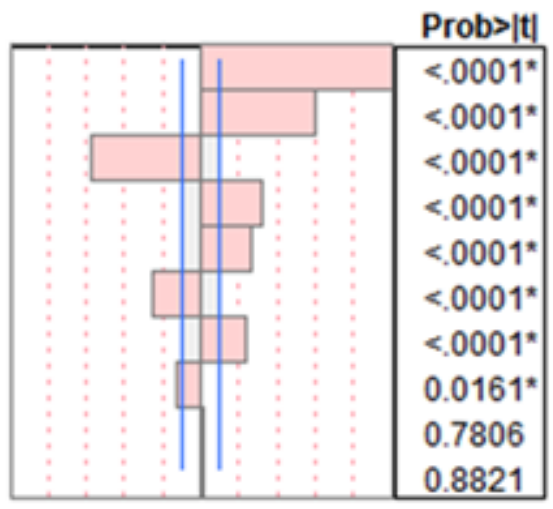

The right column in the table indicates P-values which are the lowest level of significance that would lead to the rejection of the null hypothesis $\left(\right.$ Ho: $\left.\beta_{j}=0\right)$. The P-values indicate what parameters are significant. Generally, P-values less than 0.05 are significant evidence that the parameter's coefficient is not zero. Parameters with a star are significant while the others are not significant. Eight of the ten inputs have a significant effect on the prediction of IRI.

As shown in Table 6.3, average axle width and mean wheel location do not significantly affect the prediction IRI at a significance level $\alpha=0.05$. The practical interpretation of this is the input recommendation for level 3 can be used for these two parameters without affecting predicted IRI.

Although regression coefficients are indicators of the importance of each parameter, they are not the best way to rank the parameters in order of importance because the parameters have different magnitudes (units). The most reasonable way to do such classification is to use SRC. Table 6.4 displays the IRI SRC for all parameters. The SRC are sorted from highest to lowest according to their absolute values. Notice that the order of the input parameters remains the same as in Table 6.3. From inspection of Table 6.4, it is possible to see what parameters have major effect on IRI and the relative importance among them. The higher the absolute value of the SRC, the greater the effect of the input parameters on IRI output from MEPDG. This finding is useful for planning data collection for calibration process and in decision making regarding what is the hierarchical level for each parameter. As an example, an agency may choose AADT, traffic growth, dual tire spacing, percent of heavy vehicles, traffic wander, and percent of trucks in the 
design lane to have level 1, operational speed to have level 2, average axle width and mean wheel location to have level 3.

TABLE 6.4 IRI Standardized Regression Coefficients for Structure 1

\begin{tabular}{|l|r|r|}
\hline \multicolumn{1}{|c|}{ Parameters } & Coefficient & \multicolumn{1}{c|}{ SRC } \\
\hline Two-way AADT & 3.02 & 0.69 \\
\hline Traffic Growth & 1.83 & 0.42 \\
\hline Dual tire spacing & -1.71 & -0.39 \\
\hline$\%$ Truck Design Direction & 0.98 & 0.22 \\
\hline \% Heaw Vehicles & 0.80 & 0.18 \\
\hline Traffic Wander & -0.77 & -0.17 \\
\hline \% Truck Design lane & 0.72 & 0.16 \\
\hline Operational Speed & -0.38 & -0.09 \\
\hline Average axle width & 0.04 & 0.01 \\
\hline Mean Wheel location & 0.02 & 0.01 \\
\hline
\end{tabular}

\subsubsection{Cracking Sensitivity to Traffic Input Parameters on Structure 1}

A multiple regression model with the input parameters in the LHS and Cracking was performed. Figure 6.4(a) compares cracking output from MEPDG to predicted cracking from the regression model. Although the regression parameters indicate a good fit, the figure shows a slight nonlinear trend that is confirmed by the curvature in the residual plot in Figure 6.4(b). Non-linearities may mislead the estimation of the P-values therefore a rank transformation, as explained in section 4.7, was performed in order to remove nonlinearities. Figure 6.4(c) compares the rank transformed cracking output from MEPDG to predicted cracking from the regression model. The scale of cracking changes because the data have been transformed. The regression parameters indicate a good fit $\left(\mathrm{P}<.0001\right.$ and $\left.\mathrm{R}^{2}=0.94\right)$ and no nonlinear trend is observed in the figure. The Figure 6.4(a) also shows that the regression model predicts negative values for MEPDG cracking smaller than $0.05 \%$. This is a very small amount of cracking in the pavement structure. Figure 6.4(d) shows the regression fit after dropping MEPDG cracking values smaller than $0.05 \%$. Although the negative values are removed from the analysis, Figure 6.4(d) still shows a nonlinear trend; therefore the rank transformation approach was followed in the sensitivity analysis. 


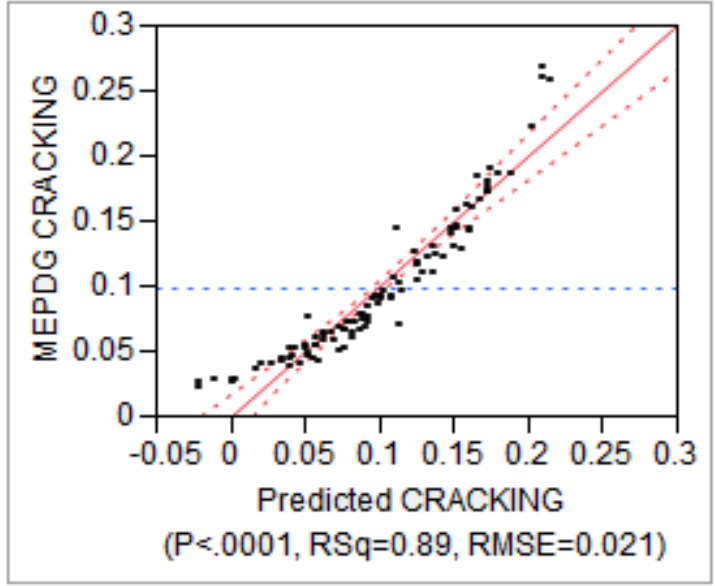

(a)

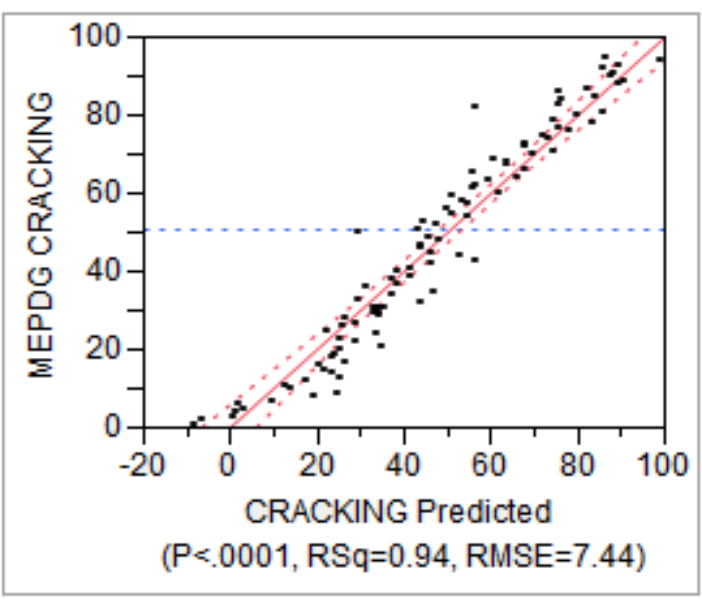

(C)

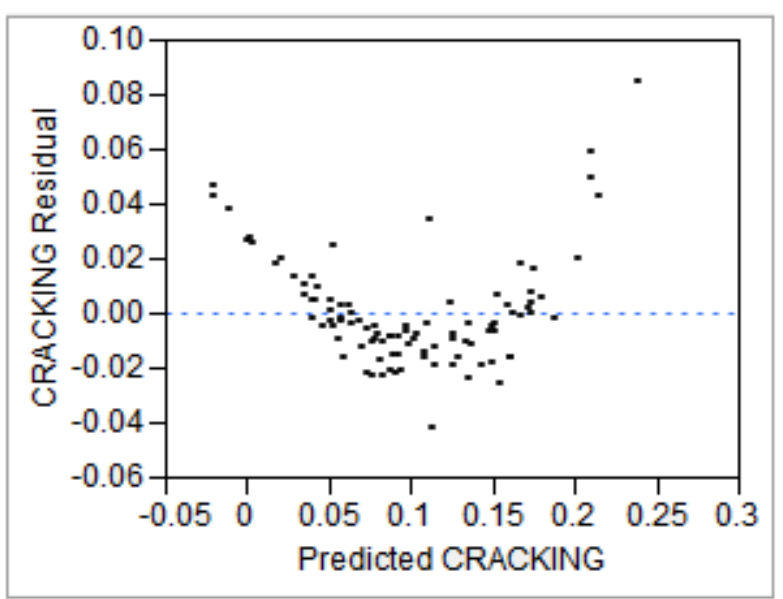

(b)

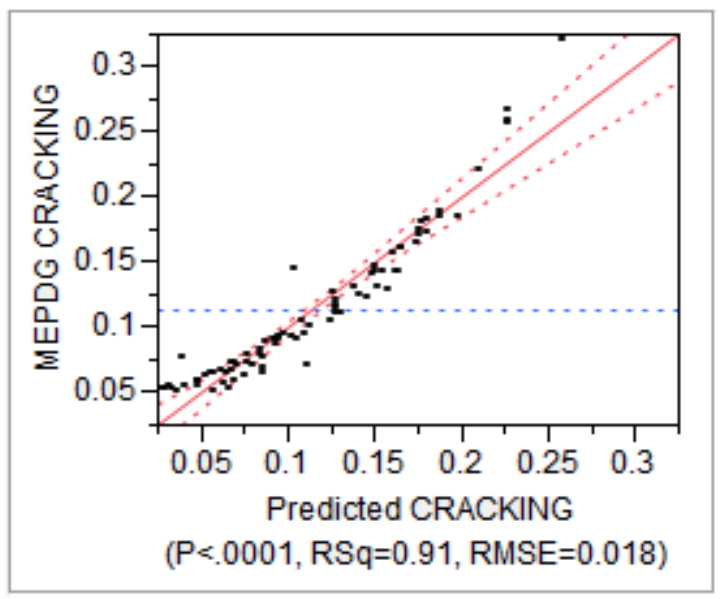

(d)

FIGURE 6.4 Cracking Actual by Predicted Plot for Structure 1

Table 6.5 shows the cracking regression coefficients. The input parameters are sorted from highest to lowest according to the absolute value of the t-ratios. Some regression coefficients have positive value while other parameters have negative regression coefficients. As with the IRI analysis, the sign of the coefficients in Table 6.5 identifies how the variable impacts the predicted cracking, i.e. a positive coefficient indicates that an increase in the variable results in more predicted cracking and vice versa. As would be expected, the amount of predicted cracking is reduced by increasing the space between dual tires and by increasing the amount of vehicle wander across the traffic lane. These parameters cannot be controlled by the pavement designer, but they need to be accurately measured. 
TABLE 6.5 Cracking Regression Coefficients for Structure 1

\begin{tabular}{|c|c|c|c|c|}
\hline Term & Coefficient & Std Error & t Ratio & Prob $>|t|$ \\
\hline Two-way AADT & 10049.497 & 388.597 & 25.86 & $<.0001^{\star}$ \\
\hline Dual tire spacing & -5.070 & 0.246 & -20.62 & $<.0001^{*}$ \\
\hline Tracffic Growth & 1.336 & 0.092 & 14.55 & $<.0001^{*}$ \\
\hline$\%$ Heavy Vehicles & 1.879 & 0.259 & 7.26 & $<.0001^{*}$ \\
\hline Traffic Wander & -0.460 & 0.078 & -5.89 & $<.0001^{\star}$ \\
\hline$\%$ Truck Design lane & 1.915 & 0.325 & 5.89 & $<.0001^{\star}$ \\
\hline$\%$ Truck Design Direction & 1.319 & 0.260 & 5.08 & $<.0001^{*}$ \\
\hline Operational Speed & -1.149 & 0.389 & -2.95 & $0.0041^{*}$ \\
\hline Mean Wheel location & 0.122 & 0.410 & 0.30 & 0.7675 \\
\hline Average axle width & -0.005 & 0.026 & -0.20 & 0.8381 \\
\hline
\end{tabular}

The right column in the Table 6.5 shows P-values. Mean wheel location and average axle width are not significant at a significance level $\alpha=0.05$. Using level 3 input values for these parameters does not affect significantly predicted cracking outputs from MEPDG. Two-way AADT has large regression coefficient and standard error. Collinearity in the data may lead to these results but multivariate analysis did not show collinearity. Rank transformation with large number of runs may lead to large coefficients because the large difference in the input values. This is the reason why SRC is a betters approach.

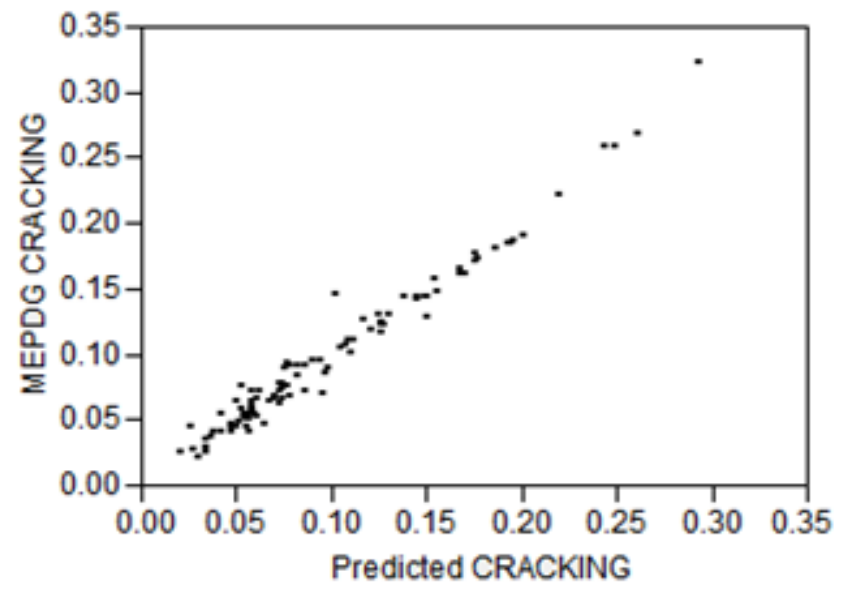

\begin{tabular}{|l|r|}
\hline \multicolumn{1}{|c|}{ Parameter } & \multicolumn{1}{|c|}{ Effect } \\
\hline Two-way AADT & 0.359 \\
\hline Dual tire spacing & 0.278 \\
\hline Tracffic Growth & 0.117 \\
\hline$\%$ Heav Vehicles & 0.041 \\
\hline$\%$ Truck Design Direction & 0.031 \\
\hline Traffic Wander & 0.024 \\
\hline$\%$ Truck Design lane & 0.023 \\
\hline Operational Speed & 0.005 \\
\hline Mean Wheel location & 0.000 \\
\hline Average axle width & 0.000 \\
\hline
\end{tabular}

FIGURE 6.5 Verification of Cracking Sensitivity by a Gaussian Process

A Gaussian process was used to verify the result from the rank transformation. Cracking data were fitted to a stochastic Gaussian process, Figure 6.5. The points in Figure 6.5 are close to the 45 degree diagonal line showing that the Gaussian process does a good job of predicting MEPDG Cracking. The advantage of this approach is that the Gaussian process is not affected by 
nonlinearities. Gaussian process does not calculate P-values which are used in this research to define the recommended hierarchical levels for the input parameters. Therefore this approach is used in this research as a means of verification in cases of nonlinearities in the data. In this approach, the total effect is divided into individual effects of each parameter as shown in the figure. (e.g., the highest effect is due to two-way AADT which main effect explains $35.9 \%$ of the total variability in MEPDG cracking predictions). Comparison between the results of the rank transformation and the Gaussian process reveals that the results are similar. Only percent of trucks in the design direction moved two places up over traffic wander and percent of truck in the design lane but the values of the SRC of these three parameters are close (Table 6.6). In general, rank transformation did a good job in identifying the most sensitive parameters.

Table 6.6 displays the cracking SRC sorted from highest to lowest according to their absolute values. The order of the input parameters remains the same as in Table 6.5. The higher the absolute value of the standardized regression coefficient, higher the effect of the input parameter on the predicted cracking output from MEPDG.

TABLE 6.6 Cracking Standardized Regression Coefficients for Structure 1

\begin{tabular}{|l|r|r|}
\hline \multicolumn{1}{|c|}{ Parameters } & Coefficient & \multicolumn{1}{c|}{ SRC } \\
\hline Two-way AADT & 10049.497 & 0.670 \\
\hline Dual tire spacing & -5.070 & -0.534 \\
\hline Tracffic Growth & 1.336 & 0.382 \\
\hline \% Heaw Vehicles & 1.879 & 0.188 \\
\hline TrafficWander & -0.460 & -0.153 \\
\hline \% Truck Design lane & 1.915 & 0.153 \\
\hline \% Truck Design Direction & 1.319 & 0.132 \\
\hline Operational Speed & -1.149 & -0.077 \\
\hline Mean Wheel location & 0.122 & 0.008 \\
\hline Average axle width & -0.005 & -0.005 \\
\hline
\end{tabular}

\subsubsection{Rutting Sensitivity to Traffic Input Parameters on Structure 1}

A multiple regression model among the input parameters in the LHS and rutting output from MEPDG was performed. Figure 6.6 presents the plot of actual rutting from MEPDG by predicted rutting from the regression model. The regression model is significant, P-value < 0.0001 . This result is confirmed by the multiple regression coefficient, $R^{2}=0.89$. The standard deviation is small compared to the magnitude of rutting values, $\mathrm{RMSE}=0.023$. 


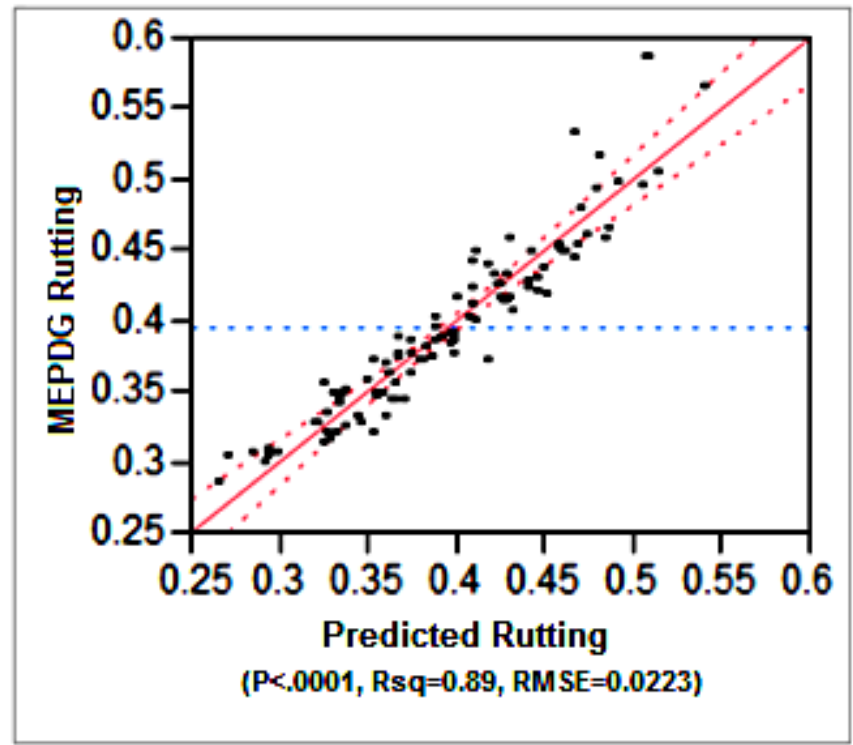

FIGURE 6.6 Rutting Actual by Predicted Plot for Structure 1

Table 6.7 displays the rutting regression coefficients. The input parameters are sorted from highest to lowest according to the absolute value of the t-ratio. The parameters with negative coefficients, dual tire spacing, traffic wander, and operational speed have inverse relationships with predicted rutting. This result is similar to the result found for IRI sensitivity. IRI performance model (equation 2.23) shows that IRI dependents on cracking, rutting, and site factor. Site factor was constant for this computer experiment and this structure had low predicted cracking. So, the similarities in IRI and rutting results are expected.

TABLE 6.7 Rutting Regression Coefficients for Structure 1

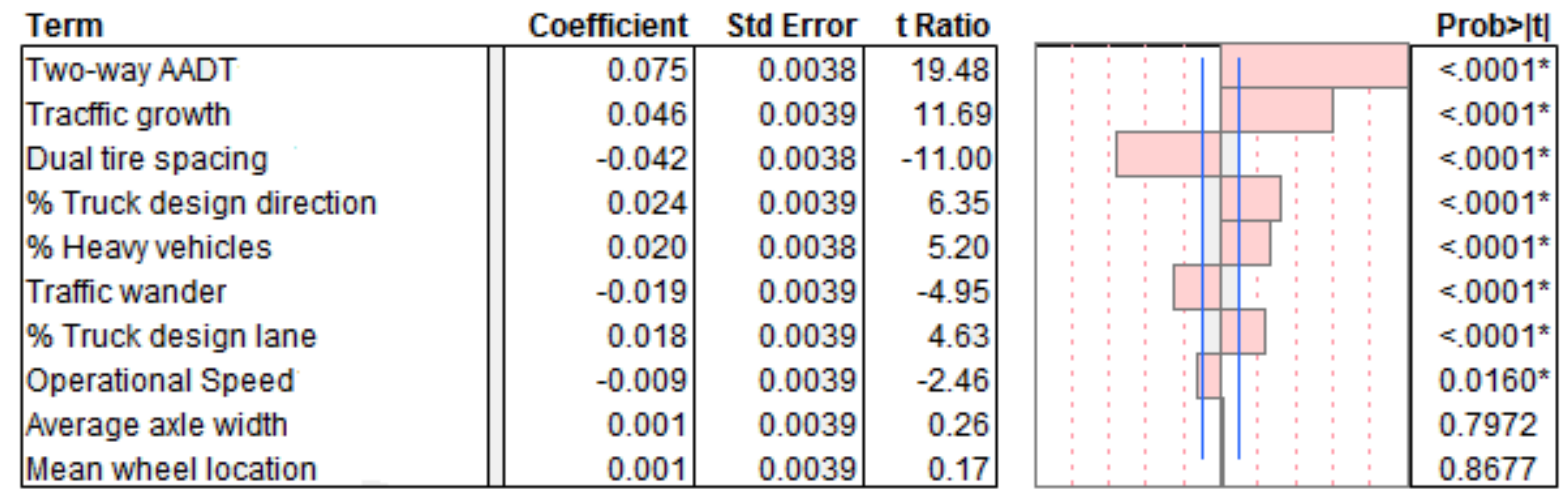


The P-values in Table 6.7 indicate that average axle width and mean wheel location are not significant at a significance level $\alpha=0.05$. Having level 3 input values for these parameters does not affect rutting outputs from MEPDG. Again, this result is similar to the IRI result.

Table 6.8 displays the rutting SRC sorted from highest to lowest according to their absolute values. The order of the input parameters remains the same as Table 6.7. The higher the absolute value of the standardized regression coefficient, higher the effect of the input parameter in rutting output from MEPDG. Two-way AADT, traffic growth, dual tire spacing, and percent of truck in the design direction are the most sensitive parameters in rutting. Percent of heavy vehicles, traffic wander, and percent of trucks in the design lane have intermediate effect with very close magnitude of standardized regression coefficients. Operational speed has much smaller effect. Average axle width and mean wheel location do not affect rutting output from MEPDG.

TABLE 6.8 Rutting Standardized Regression Coefficients for Structure 1

\begin{tabular}{|l|r|r|}
\hline \multicolumn{1}{|c|}{ Parameters } & Coefficient & \multicolumn{1}{c|}{ SRC } \\
\hline Two-way AADT & 0.075 & 0.687 \\
\hline Tracffic Growth & 0.046 & 0.418 \\
\hline Dual tire spacing & -0.042 & -0.388 \\
\hline \% Truck Design Direction & 0.024 & 0.224 \\
\hline$\%$ Heaw Vehicles & 0.020 & 0.183 \\
\hline Traffic Wander & -0.019 & -0.175 \\
\hline \% Truck Design lane & 0.018 & 0.164 \\
\hline Operational Speed & -0.009 & -0.087 \\
\hline Average axle width & 0.001 & 0.009 \\
\hline Mean Wheel location & 0.001 & 0.006 \\
\hline
\end{tabular}

\subsection{Results of Traffic Sensitivity on Structure 2}

\subsubsection{IRI Sensitivity to Traffic Input Parameters on Structure 2}

A multiple regression model among the input parameters in the LHS and IRI output from MEPDG was performed. Figure 6.7(a) compares the IRI from MEPDG to IRI predicted from the regression model. Although the fit is good, there is non-linear trend that is confirmed by the curvature of the residuals in the residuals plot in Figure 6.7(b). A rank transformation was performed to remove nonlinearities from the data, Figure 6.7(c). The regression is significant, Pvalue $<0.0001$ and $\mathrm{R}^{2}=0.92$. The rank transformed data were used in the analysis. 


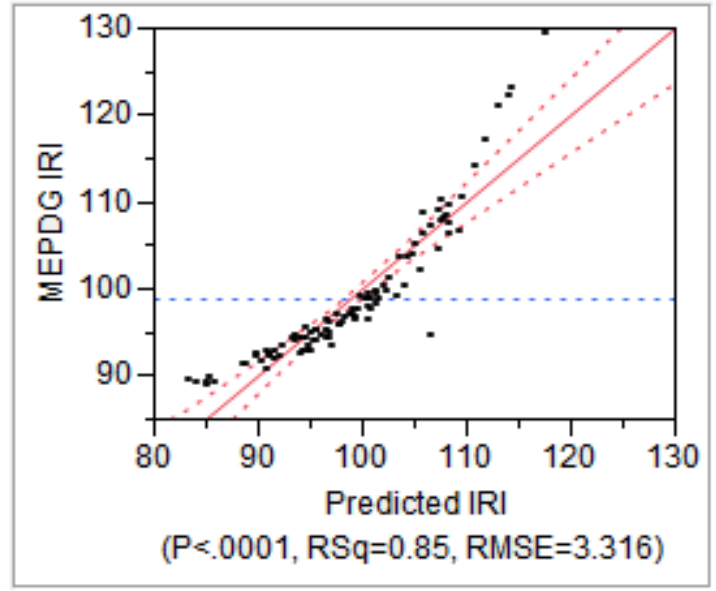

(a)

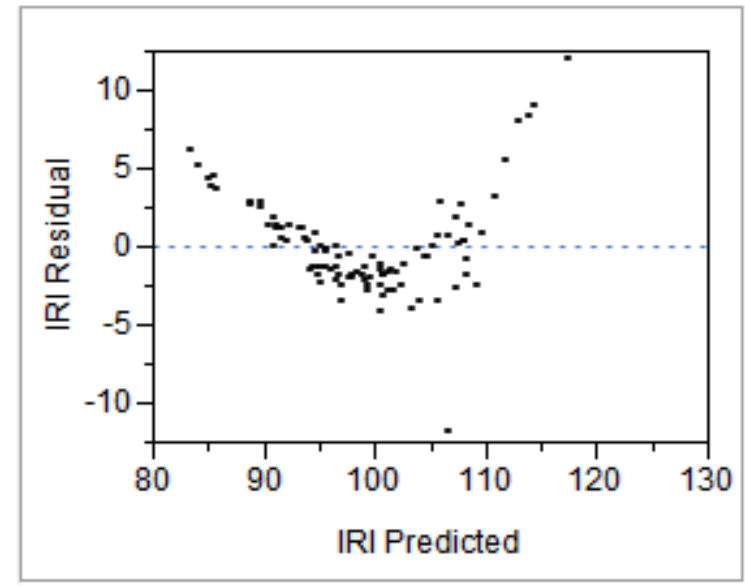

(b)

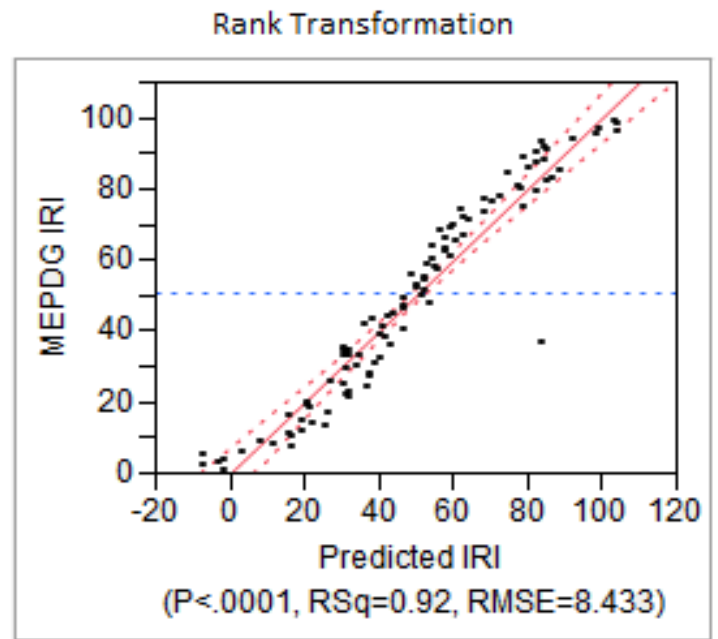

(c)

FIGURE 6.7 IRI Actual by Predicted Plot for Structure 2

Table 6.9 shows the IRI regression coefficients, standard error, t-ratios, and P-values. The input parameters were sorted from highest to lowest according to the absolute value of the tratios. Dual tire spacing, traffic wander, and operational speed have negative regression coefficients. Average axle width also had negative SRC but the value is close to zero. Operational speed, average axle width, and mean wheel location do not significantly affect the IRI output. Two-way AADT has large regression coefficient and standard error. Collinearity in the data may lead to large coefficients but multivariate analysis did not show collinearity. Rank transformation with large number of runs may lead to large coefficients because the large difference in the input values. This is the reason why SRC are a betters approach. 
TABLE 6.9 IRI Regression Coefficients for Structure 2

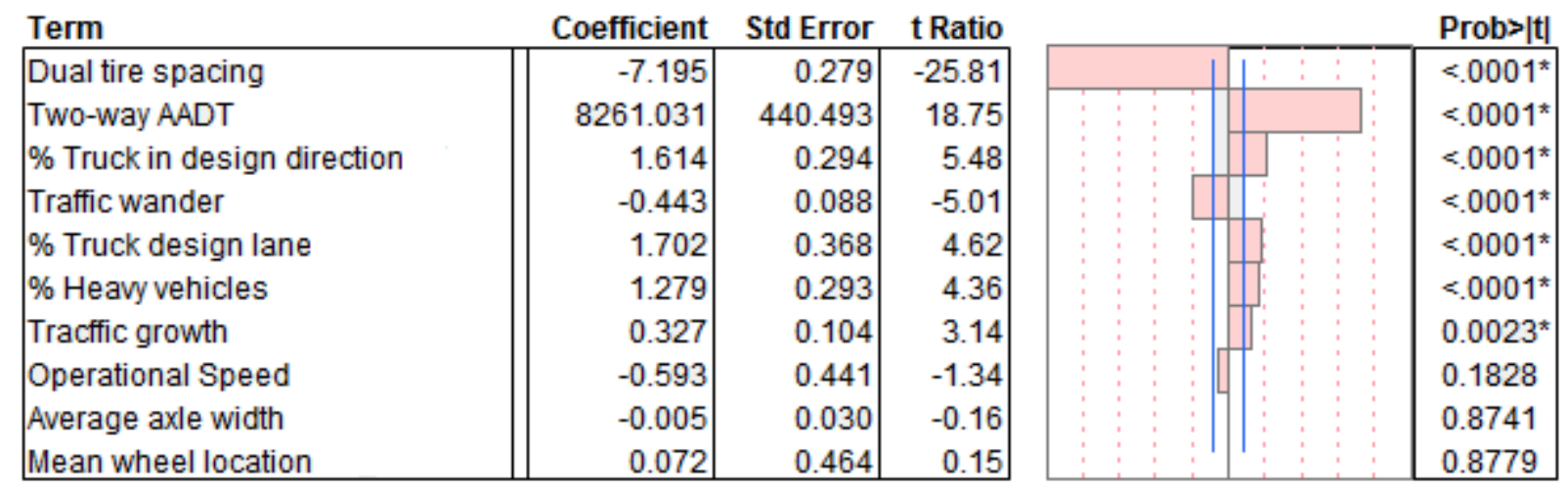

Table 6.10 displays the IRI SRC sorted from highest to lowest according to their absolute values. The relative importance among parameters is known by comparing the magnitude of the standardized coefficients. Table 6.10 shows that the highest effects on IRI output from MEPDG are due to dual tire spacing, two-way AADT, percent of truck in the design direction, and traffic wander. In this case, dual tire spacing was more sensitive than two-way AADT. This confirms that dual tire spacing has a high effect on pavement performance and structure 2 is even more sensitive to this parameter than structure 1.

TABLE 6.10 IRI Standardized Regression Coefficients for Structure 2

\begin{tabular}{|l|r|r|}
\hline \multicolumn{1}{|c|}{ Parameters } & Coefficient & \multicolumn{1}{c|}{ SRC } \\
\hline Dual tire spacing & -7.195 & -0.757 \\
\hline Two-way AADT & 8261.031 & 0.551 \\
\hline$\%$ Truck in design direction & 1.614 & 0.161 \\
\hline Traffic wander & -0.443 & -0.148 \\
\hline$\%$ Truck design lane & 1.702 & 0.136 \\
\hline \% Heaw vehicles & 1.279 & 0.128 \\
\hline Tracfic growth & 0.327 & 0.093 \\
\hline Operational Speed & -0.593 & -0.040 \\
\hline Average axle width & -0.005 & -0.005 \\
\hline Mean wheel location & 0.072 & 0.005 \\
\hline
\end{tabular}

IRI traffic data was fitted to a Gaussian process (Figure 6.8) to verify the results of the rank transformation. Good fit of the Gaussian model in predicting MEPDG IRI is inferred from the proximity of the points to the 45 degree diagonal. Comparison among the two approaches shows that the results are very consistent. The order of importance of the effects is very similar in both approaches. The three most significant parameters coincided in their positions. In the 
significant parameters only percent of heavy vehicles moved two places up. Mean wheel location and average axle width interchanged positions but these parameters are not significant.

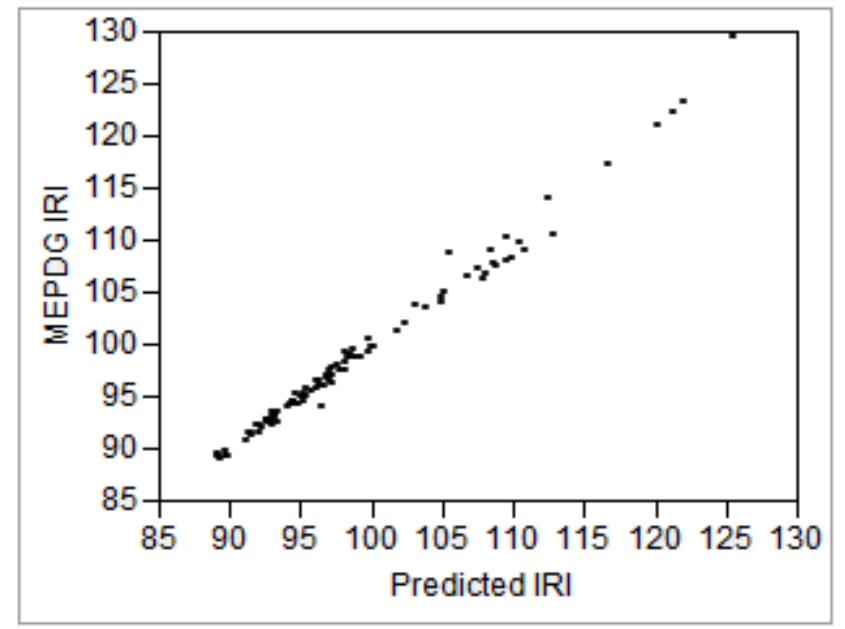

\begin{tabular}{|l|r|}
\hline \multicolumn{1}{|c|}{ Parameters } & \multicolumn{1}{c|}{ Effect } \\
\hline Dual tire spacing & 0.593 \\
\hline Two-way AADT & 0.244 \\
\hline \% Truck in design direction & 0.026 \\
\hline \% Heavy vehicles & 0.019 \\
\hline Traffic wander & 0.015 \\
\hline \% Truck design lane & 0.012 \\
\hline Tracffic growth & 0.010 \\
\hline Operational Speed & 0.001 \\
\hline Mean wheel location & 0.000 \\
\hline Average axle width & 0.000 \\
\hline
\end{tabular}

FIGURE 6.8 Verification of IRI Sensitivity with a Gaussian Process

\subsubsection{Cracking Sensitivity to Traffic Input Parameters on Structure 2}

A multiple regression model with the input parameters in the LHS and cracking output from MEPDG was performed. Figure 6.9(a) compares cracking output from MEPDG to cracking predicted from the regression model. Some evidence of nonlinear trend is identified in the figure which is confirmed by the curvature in the residuals plot in Figure 6.9(b). The regression model also predicts negative cracking for MEPDG cracking outputs smaller than 5\%. Two approaches were followed to investigate the sensitivity of cracking. The first approach was to performed a rank transformation. The second approach was to drop MEPDG cracking outputs smaller than 5\%. Figure 6.10(a) compares the rank transformed cracking from MEPDG with cracking predicted from the regression model. $\mathrm{P}$-value $<0.0001$ and $\mathrm{R}^{2}=0.94$ indicate a good fit and a non-linear trend is observed in the figure. The nonlinearities were removed but some negative numbers are still present. Figure 6.10(b) compares the cracking from MEPDG, without values smaller than 5\%, with cracking predicted from the regression model. The regression model shows a linear trend, the negative values were removed, and the regression coefficient is good $\left(\mathrm{R}^{2}=0.90\right)$. The sensitivity analysis was performed for both situations rank transformation and dropping of MEPDG cracking smaller than $5 \%$. 


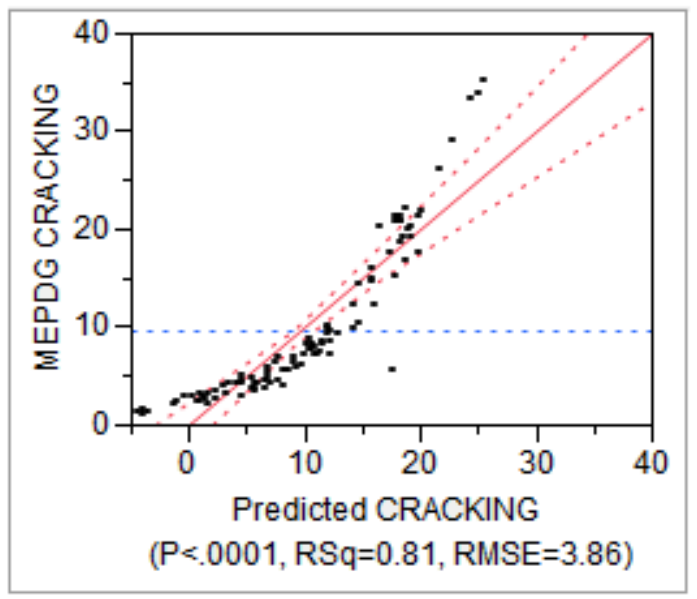

(a)

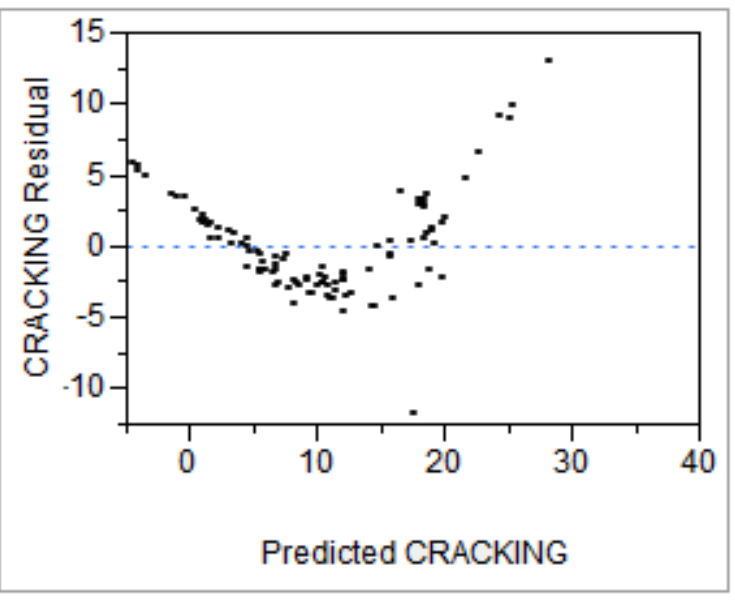

(b)

FIGURE 6.9 Cracking Actual by Predicted Plot for Structure 2

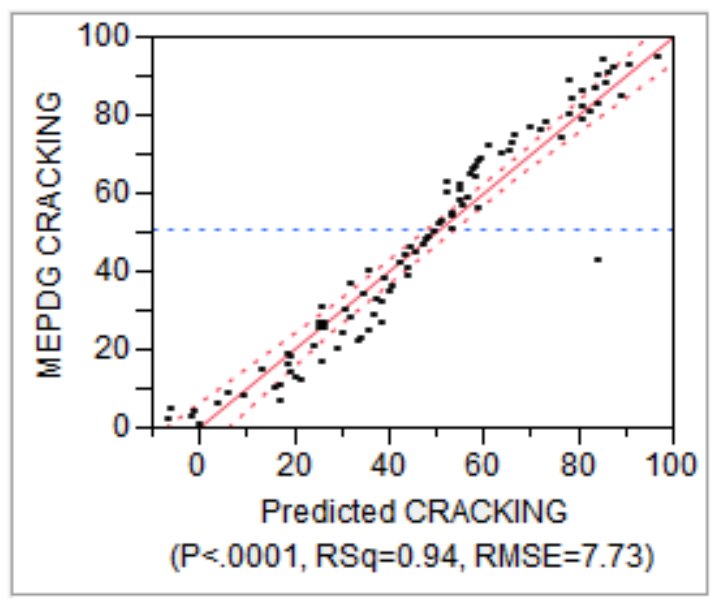

(a)

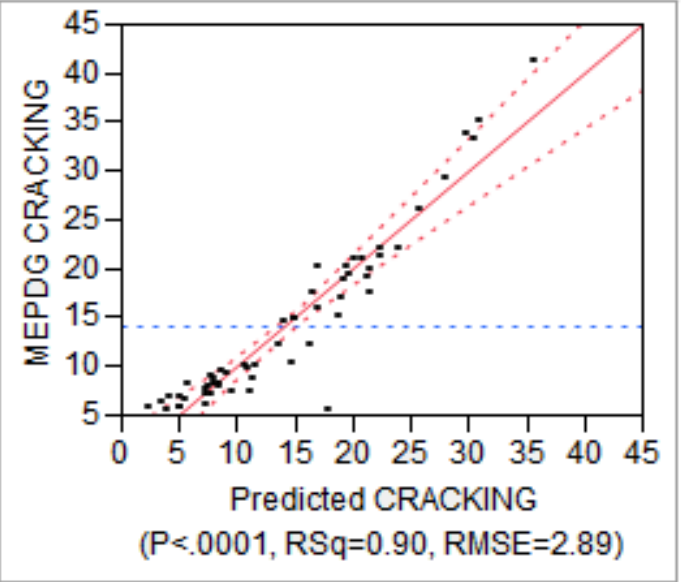

(b)

FIGURE 6.10 Cracking Actual by Predicted Plot for Two Approaches of Structure 2

Table 6.11 shows the cracking regression coefficients, standard error, t-ratio, and Pvalues for the rank transformation approach. The input parameters are sorted from highest to lowest according to the absolute value of the t-ratios. Dual tire spacing, traffic wander, average axle width, and operational speed have negative coefficients. Operational speed, mean wheel location, and average axle width do not significantly affect the cracking output from MEPDG. This result is similar to the result found for IRI. Two-way AADT has large regression coefficient and standard error. As explained above, collinearity in the data may lead to these results 
nevertheless multivariate analysis did not reveal collinearity. Rank transformation with large number of runs may lead to large coefficients because the large difference in input values. This is the reason why SRC is a betters approach.

TABLE 6.11 Cracking Regression Coefficients for Structure 2

\begin{tabular}{|c|c|c|c|c|}
\hline Term & Estimate & Std Error & t Ratio & Prob $>|t|$ \\
\hline Dual tire spacing & -7.710 & 0.255 & -30.19 & $<.0001^{\star}$ \\
\hline Two-way AADT & 7394.946 & 403.532 & 18.33 & $<.0001^{*}$ \\
\hline$\%$ Truck in design direction & 1.413 & 0.270 & 5.24 & $<.0001^{*}$ \\
\hline Traffic wander & -0.421 & 0.081 & -5.20 & $<.0001^{\star}$ \\
\hline$\%$ Truck design lane & 1.524 & 0.337 & 4.52 & $<.0001^{*}$ \\
\hline$\%$ Heavy vehicles & 1.210 & 0.269 & 4.50 & $<.0001^{*}$ \\
\hline Tracffic growth & 0.282 & 0.095 & 2.95 & $0.0040^{*}$ \\
\hline Operational Speed & -0.332 & 0.404 & -0.82 & 0.4131 \\
\hline Mean wheel location & 0.129 & 0.425 & 0.30 & 0.7620 \\
\hline Average axle width & -0.004 & 0.027 & -0.13 & 0.8966 \\
\hline
\end{tabular}

The magnitudes of SRC in Table 6.12 show that the highest effects on cracking output are due to dual tire spacing, two-way AADT, and percent of truck in the design direction. Traffic wander, percent of truck in the design lane, and traffic growth have effects with close values of SRC. Again, dual tire spacing was more sensitive than two-way AADT.

TABLE 6.12 Cracking Standardized Regression Coefficients for Structure 2

\begin{tabular}{|l|r|r|}
\hline \multicolumn{1}{|c|}{ Term } & Coefficient & \multicolumn{1}{c|}{ SRC } \\
\hline Dual tire spacing & -7.710 & -0.812 \\
\hline Two-way AADT & 7394.946 & 0.493 \\
\hline \% Truck in design direction & 1.413 & 0.141 \\
\hline Traffic wander & -0.421 & -0.140 \\
\hline$\%$ Truck design lane & 1.524 & 0.122 \\
\hline \% Heawy vehicles & 1.210 & 0.121 \\
\hline Tracfic growth & 0.282 & 0.080 \\
\hline Operational Speed & -0.332 & -0.022 \\
\hline Mean wheel location & 0.129 & 0.008 \\
\hline Average axle width & -0.004 & -0.004 \\
\hline
\end{tabular}

Table 6.13 shows the cracking regression coefficients, standard error, t-ratio, and Pvalues for the approach of dropping values smaller than 5\%. Although the values of regression coefficients are different, the importance of parameters and the sign of the regression coefficients of the significant parameters are the same as in the previous approach. Only average axle width 
moved 2 positions up over operational speed and mean wheel location, but these parameters are not significant. The same was found with the SRC in table 6.14.

TABLE 6.13 Cracking Regression Coefficients for Structure 2 (Values > 5\%)

Term
\begin{tabular}{|l|r|r|r|}
\hline Dual tire spacing & -17.663 & 0.925 & -19.10 \\
Two-way AADT & 95.962 & 7.700 & 12.46 \\
\% Truck in design direction & 4.187 & 0.655 & 6.40 \\
Traffic wander & -4.119 & 0.650 & -6.34 \\
\% Truck design lane & 3.292 & 0.663 & 4.97 \\
\% Heavy vehicles & 2.477 & 0.674 & 3.68 \\
Tracffic growth & 2.313 & 0.655 & 3.53 \\
Average axle width & 0.874 & 0.661 & 1.32 \\
Operational Speed & -0.381 & 0.648 & -0.59 \\
Mean wheel location & -0.192 & 0.640 & -0.30 \\
\hline
\end{tabular}

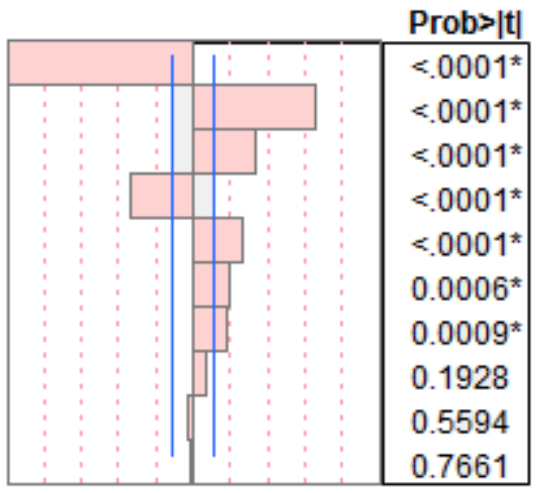

TABLE 6.14 Cracking Standardized Regression Coefficients for Structure 2 (Values > 5\%)

\begin{tabular}{|l|r|r|}
\hline \multicolumn{1}{|c|}{ Term } & Estimate & \multicolumn{1}{c|}{ SRC } \\
\hline Dual tire spacing & -17.66 & -0.95 \\
\hline Two-way AADT & 95.96 & 0.60 \\
\hline$\%$ Truck in design direction & 4.19 & 0.29 \\
\hline Traffic wander & -4.12 & -0.29 \\
\hline$\%$ Truck design lane & 3.29 & 0.23 \\
\hline \% Heaw vehicles & 2.48 & 0.17 \\
\hline Tracffic growth & 2.31 & 0.16 \\
\hline Average axle width & 0.87 & 0.06 \\
\hline Operational Speed & -0.38 & -0.03 \\
\hline Mean wheel location & -0.19 & -0.01 \\
\hline
\end{tabular}

Cracking data was fitted to a stochastic Gaussian process (Figure 6.11) in order to verify the results of the previous approaches. The points in Figure 6.5 are close to the 45 degree diagonal line showing that the Gaussian process does a good job of predicting MEPDG Cracking. Comparison among the three approaches shows that the results are very consistent. The order of importance of the effects is exactly equal in the approaches with rank transformation and Gaussian process. 


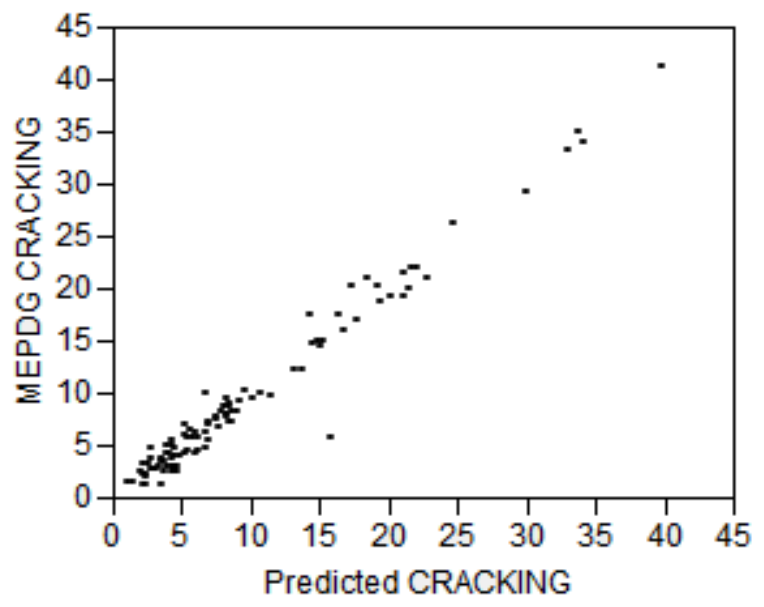

\begin{tabular}{|l|r|}
\hline \multicolumn{1}{|c|}{ Parameter } & Effect \\
\hline Dual tire spacing & 0.608 \\
\hline Two-way AADT & 0.187 \\
\hline \% Truck in design direction & 0.025 \\
\hline Traffic wander & 0.018 \\
\hline \% Truck design lane & 0.015 \\
\hline \% Heaw vehicles & 0.013 \\
\hline Tractic growth & 0.006 \\
\hline Operational Speed & 0.000 \\
\hline Mean wheel location & 0.000 \\
\hline Average axle width & 0.000 \\
\hline
\end{tabular}

FIGURE 6.11 Verification of Cracking Sensitivity with a Gaussian Process

\subsubsection{Rutting Sensitivity to Traffic Input Parameters on Structure 2}

A comparison of rutting from MEPDG to cracking predicted from the regression model for structure 2 is shown in Figure 6.12. The P-value shows that the regression model is highly significant ( $\mathrm{P}$-value $<0.0001)$. This result is confirmed by the multiple regression coefficient, $\mathrm{R}^{2}$ $=0.93$. The Standard deviation, $\mathrm{RMSE}=0.02$ is small compared to the magnitude of rutting values.

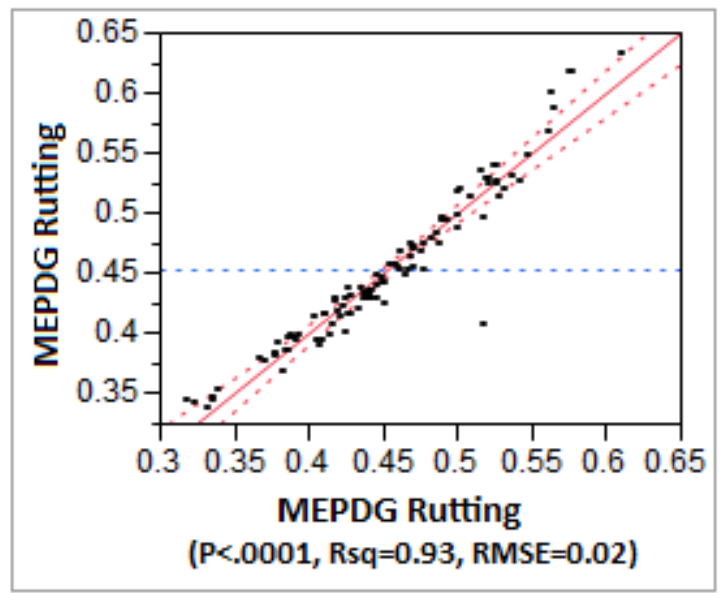

FIGURE 6.12 Rutting Actual by Predicted Plot for Structure 2

Table 6.15 shows the rutting regression coefficients, standard error, t-ratios, and P-values for structure 2. Dual tire spacing, traffic wander, and operational speed have negative 
coefficients. Operational speed, average axle width, and mean wheel location do not significantly affect the rutting output from MEPDG.

TABLE 6.15 Rutting Regression Coefficients for Structure 2

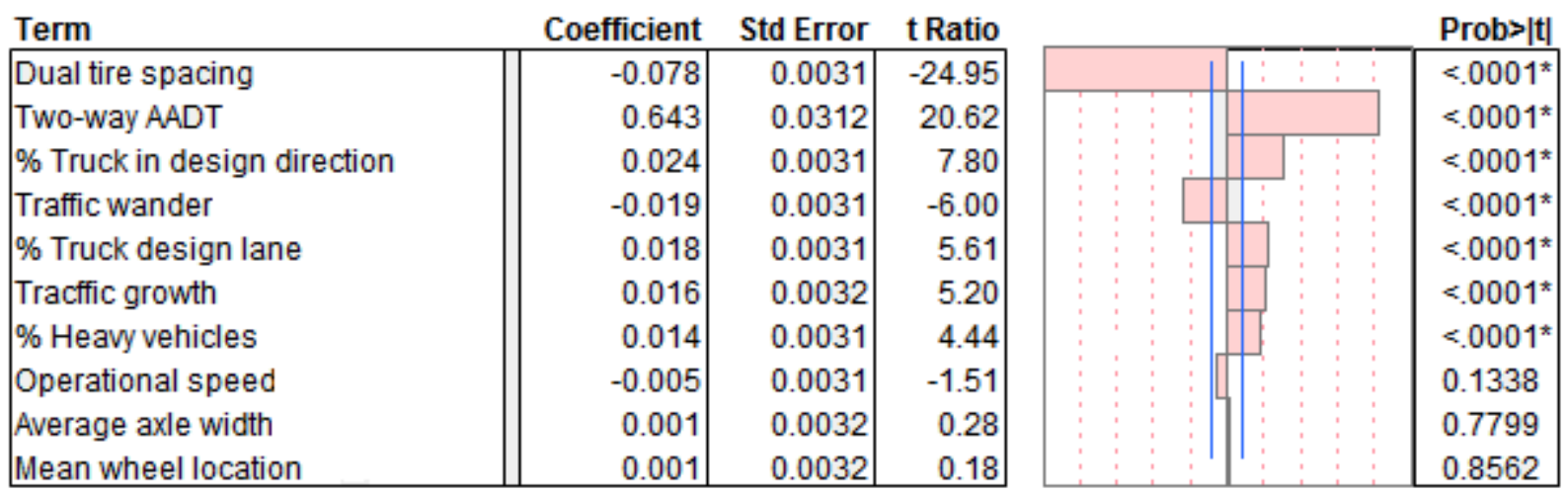

Table 6.16 displays the rutting SRC for structure 2 that are sorted from highest to lowest according to their absolute values. Table 6.14 shows that the highest effects on rutting are due to dual tire spacing, two-way AADT, and percent of trucks in the design direction. Traffic wander, percent of trucks in the design lane, and traffic growth have also significant effects and very close values of SRC showing that their effects have similar magnitudes.

TABLE 6.16 Rutting Standardized Regression Coefficients for Structure 2

\begin{tabular}{|l|r|r|}
\hline \multicolumn{1}{|c|}{ Parameters } & Coefficient & \multicolumn{1}{c|}{ SRC } \\
\hline Dual tire spacing & -0.08 & -0.71 \\
\hline Two-way AADT & 0.64 & 0.58 \\
\hline$\%$ Truck in design direction & 0.02 & 0.22 \\
\hline Traffic wander & -0.02 & -0.17 \\
\hline$\%$ Truck design lane & 0.02 & 0.16 \\
\hline Tracfic growth & 0.02 & 0.15 \\
\hline \% Heaw vehicles & 0.01 & 0.13 \\
\hline Operational Speed & 0.00 & -0.04 \\
\hline Average axle width & 0.00 & 0.01 \\
\hline Mean wheel location & 0.00 & 0.01 \\
\hline
\end{tabular}

\subsection{Summary of Results of Traffic Sensitivity}

In general, MEPDG predicted rutting correlates well with traffic input parameters. Nonlinearities were found for cracking in structure 1 and for IRI and cracking in structure 2. Rank transformations removed nonlinearities and improved the multiple correlation coefficients, $\mathrm{R}^{2}$. The good regression models explain much of the variation of the MEPDG outputs in 
response to their inputs. Summaries of SRC for IRI, rutting, and cracking in traffic sensitivity for structure 1 and 2 are presented in Table 6.17 and Figures 6.13 and 6.14. A star in front of parameter's bar indicates a significant effect on the MEPDG output at 0.05 level of significance. The similarity of results for IRI and rutting in structure 1 are evident in Table 6.17 and Figure 6.13. That similarity does not appear in structure 2 as seen in Figure 6.14. Although, the values of the regression coefficients are different because of the different magnitudes of the input parameters, the standardized regression coefficients are almost the same. Equation 2.23 shows IRI depends on cracking, rutting, and site factor. Site factor was constant for this computer experiment and little cracking was predicted for structure 1. So, the similarities in IRI and rutting results in structure 1 are expected. This analysis is confirmed by the results for structure 2 in which these strong similarities did not come out.

TABLE 6.17 Summary of SRC from Traffic Results

\begin{tabular}{|l|r|r|r|r|r|r|}
\cline { 2 - 7 } \multicolumn{1}{c|}{} & \multicolumn{4}{c|}{ Standardized Regression Coefficients (SRC) } \\
\cline { 2 - 7 } \multicolumn{1}{c|}{} & \multicolumn{3}{c|}{ Structure 1 } & \multicolumn{3}{c|}{ Structure 2 } \\
\hline \multicolumn{1}{c|}{ Parameters } & \multicolumn{1}{c|}{ IRI } & Rutting & Cracking & \multicolumn{1}{c|}{ IRI } & Rutting & Cracking \\
\hline Two-way AADT & 0.687 & 0.687 & 0.670 & 0.551 & 0.585 & 0.493 \\
\hline Tracfic Growth & 0.417 & 0.418 & 0.382 & 0.093 & 0.149 & 0.080 \\
\hline Dual tire spacing & -0.390 & -0.388 & -0.534 & -0.757 & -0.707 & -0.812 \\
\hline \% Truck Design Direction & 0.223 & 0.224 & 0.132 & 0.161 & 0.222 & 0.141 \\
\hline \% Heaw Vehicles & 0.182 & 0.183 & 0.188 & 0.128 & 0.126 & 0.121 \\
\hline Traffic Wander & -0.175 & -0.175 & -0.153 & -0.148 & -0.171 & -0.140 \\
\hline \% Truck Design lane & 0.165 & 0.164 & 0.153 & 0.136 & 0.160 & 0.122 \\
\hline Operational Speed & -0.087 & -0.087 & -0.077 & -0.040 & -0.043 & -0.022 \\
\hline Average axle width & 0.010 & 0.009 & -0.005 & -0.005 & 0.008 & -0.004 \\
\hline Mean Wheel location & 0.005 & 0.006 & 0.008 & 0.005 & 0.005 & 0.008 \\
\hline
\end{tabular}

For both structures the signs of the SRC are equal for all significant effects for all distresses. Two-way AADT, traffic growth, percent of trucks in design direction, percent of heavy vehicles, and percent of trucks in the design lane have positive SRC for all distresses in both structures. Dual tire spacing, traffic wander, and operational speed have negative SRC for all distresses in both structures. Some differences in sign of SRC were found between structures for average axle width and mean wheel location but the magnitude of the SRC of these parameters is very small (close to zero) and they do not affect significantly either of the MEPDG outputs in the study. The sign of very small values of SRC is not accurately predicted by the 
multiple regression models but this is negligible because parameters with a very small SRC do not significantly affect the MEPDG outputs.

Figures 6.15, 6.16, and 6.17 compare SRC of both structures. Dark bars represent Structure 1 and light bars represent structure 2. Parameters at the right side of the figure have positive SRC and parameters at the left side have negative SRC. As was shown in Table 6.17 parameters with significant effects have the same sign of SRC for both structures and all distresses. The highest effects in both structures are due to two-way AADT and dual tire spacing for all distresses. Operational speed has a significant effect for all distresses in structure 1 but is not significant for all distresses in structure 2 for all distresses. In general, mean wheel location and average axle width are not significant for all distresses.

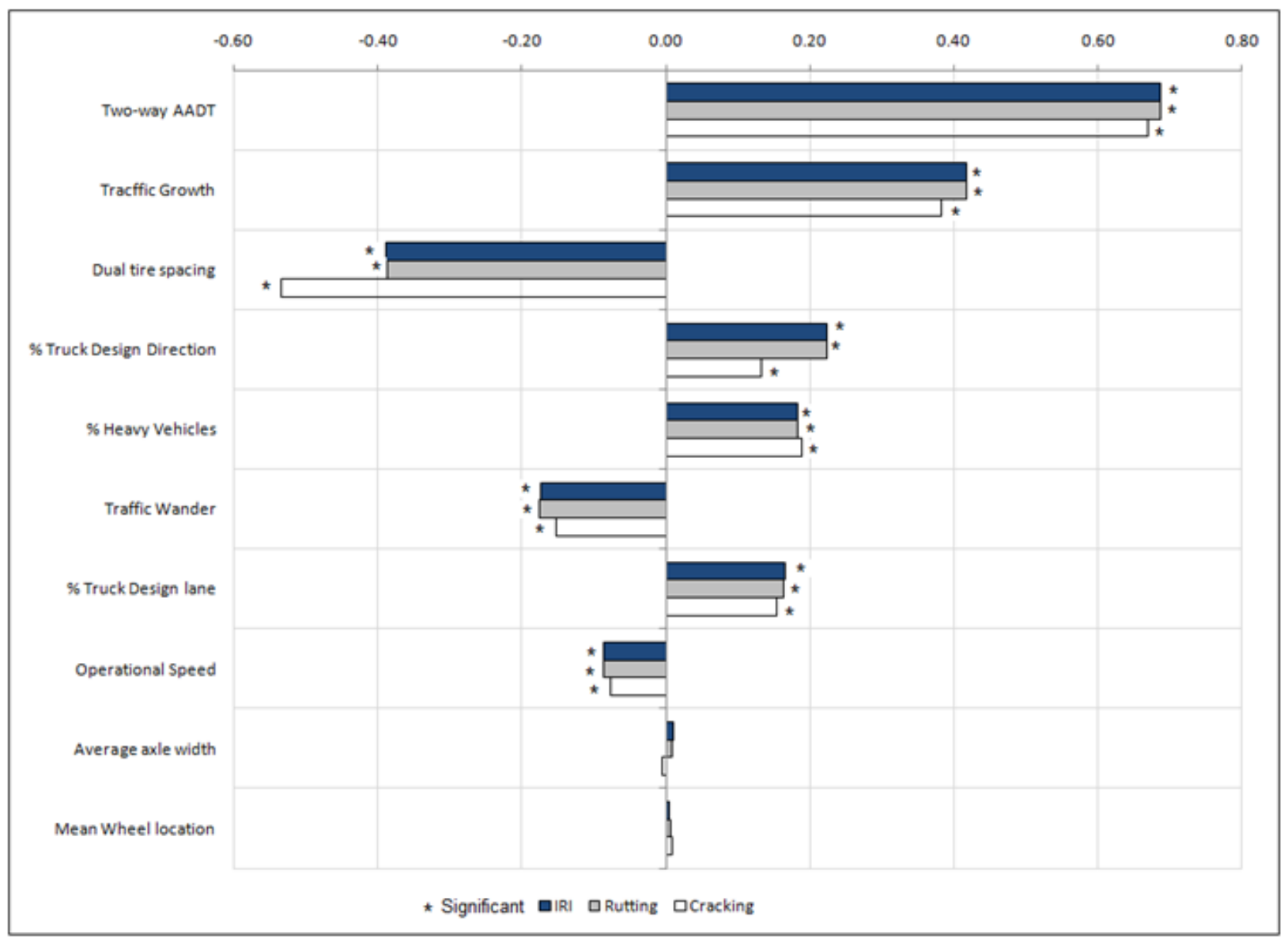

FIGURE 6.13 Standardized Regression Coefficients of Structure 1 


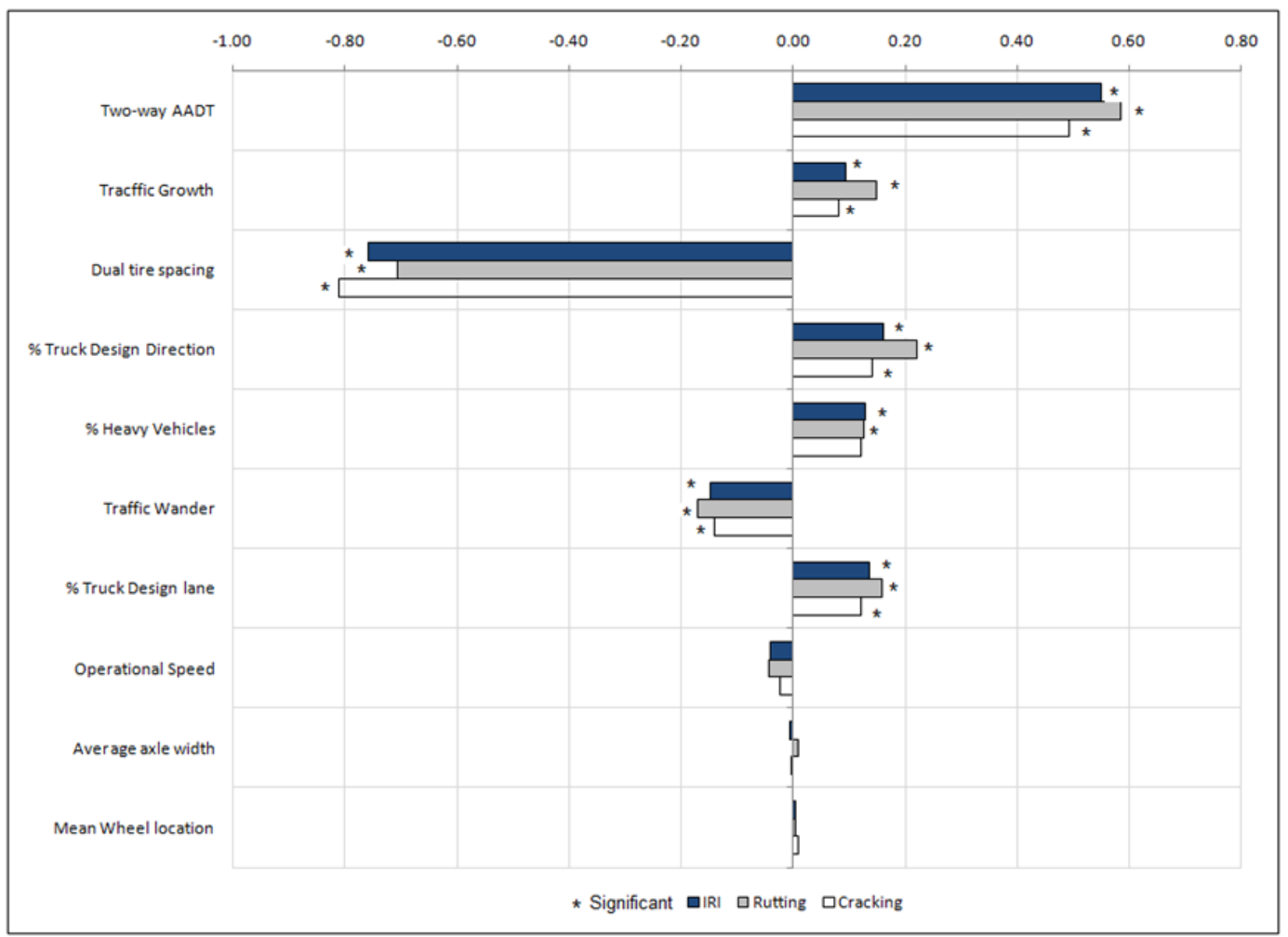

FIGURE 6.14 Standardized Regression Coefficients of Structure 2

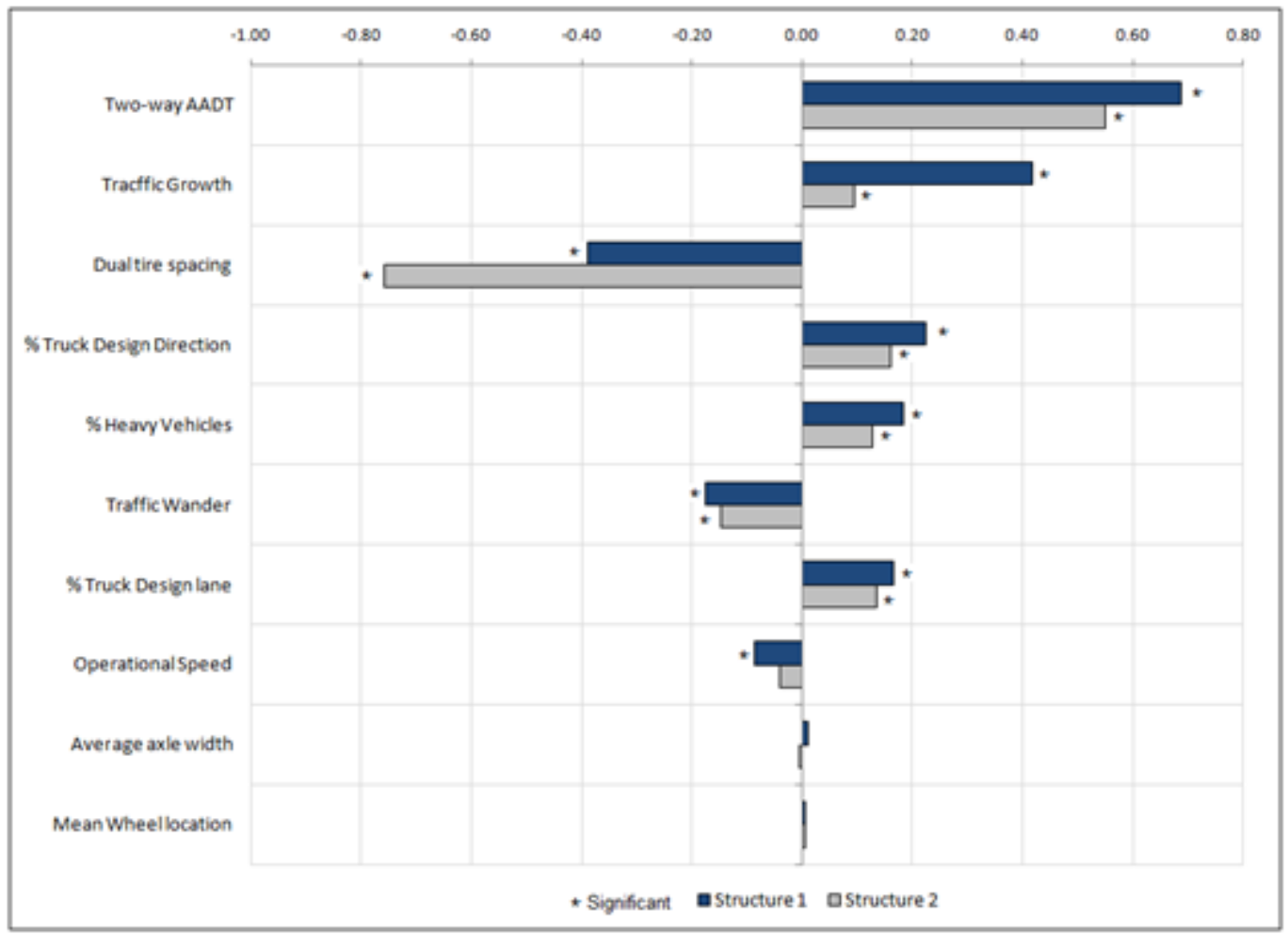

FIGURE 6.15 IRI Standardized Regression Coefficients 


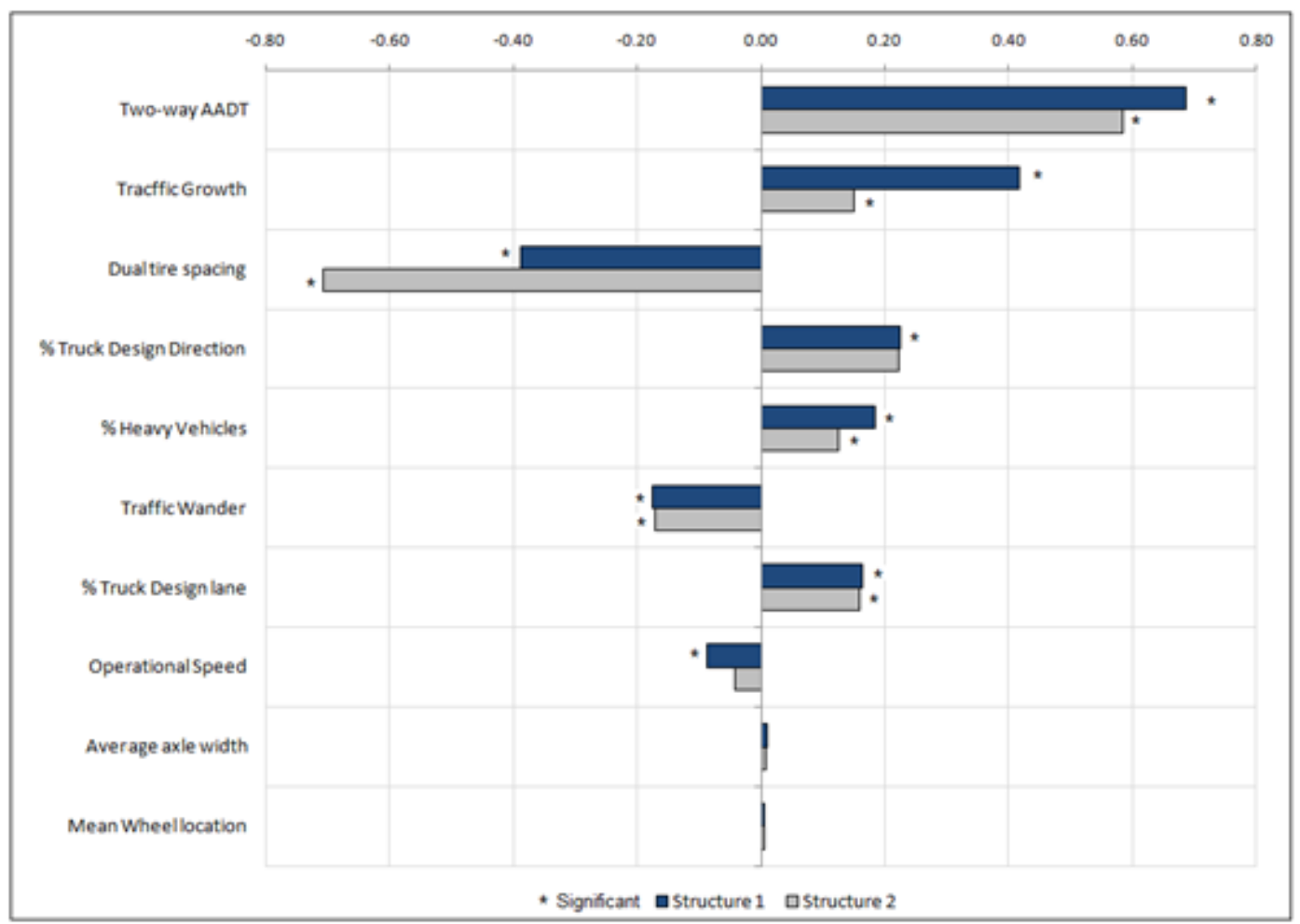

FIGURE 6.16 Rutting Standardized Regression Coefficients

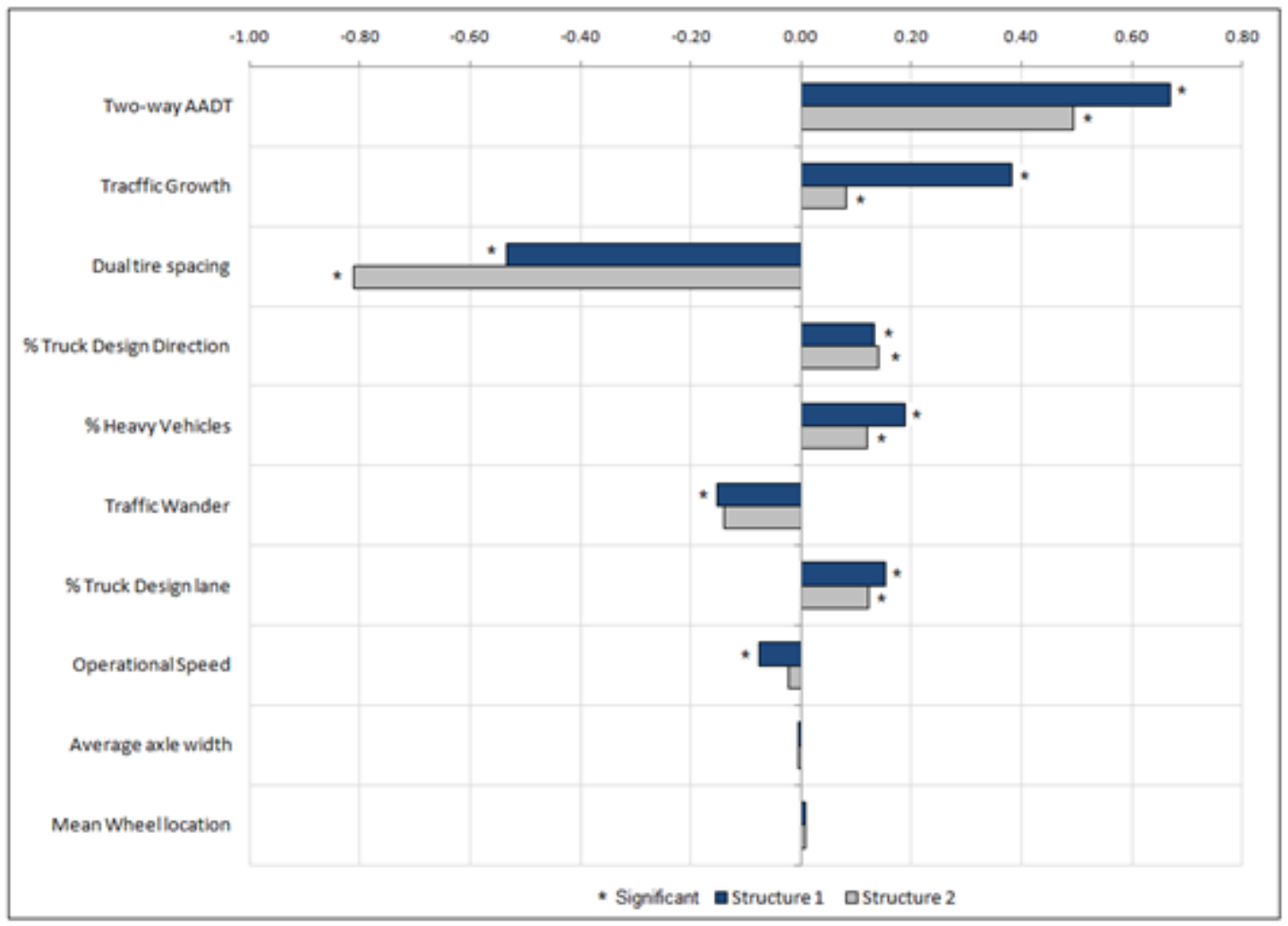

FIGURE 6.17 Cracking Standardized Regression Coefficients 


\subsection{Recommended Hierarchical Levels for Traffic Input Parameters}

The results of sensitivity analysis were used to identify recommended hierarchical levels for input parameters in this research. A defined methodology to choose hierarchical levels for input parameters in MEPDG does not exist at this time. The approach in this research was as follows;

1. Highly significant parameters with $\mathrm{P}<0.0001$ were assigned to hierarchical level 1

2. Significant parameters with $P \geq 0.0001$ were assigned to hierarchical level 2

3. No significant parameters were assigned to hierarchical level 3

This approach is based on the premise that parameters which do not affect significantly an MEPDG output may be given any reasonable input value without considerably affecting the MEPDG output. Input values for highly significant parameters have large effects on MEPDG predictions.

Table 6.18 presents the assigned hierarchical levels for traffic parameters for both structures. The hierarchical levels were assigned for each parameter and distress. The overall hierarchical levels were defined as the highest level from all distresses. 
TABLE 6.18 Recommended Hierarchical Levels for Traffic Inputs

\begin{tabular}{|l|c|c|c|c|}
\cline { 2 - 5 } \multicolumn{1}{c|}{} & \multicolumn{4}{c|}{ Structure 1 } \\
\hline \multicolumn{1}{c|}{ Parameters } & IRI & Rutting & Cracking & Overall \\
\hline Two-way AADT & 1 & 1 & 1 & 1 \\
\hline Tracffic Growth & 1 & 1 & 1 & 1 \\
\hline Dual tire spacing & 1 & 1 & 1 & 1 \\
\hline$\%$ Truck Design Direction & 1 & 1 & 1 & 1 \\
\hline$\%$ Heaw Vehicles & 1 & 1 & 1 & 1 \\
\hline Traffic Wander & 1 & 1 & 1 & 1 \\
\hline$\%$ Truck Design lane & 1 & 1 & 1 & 1 \\
\hline Operational Speed & 2 & 2 & 2 & 2 \\
\hline Average axle width & 3 & 3 & 3 & 3 \\
\hline Mean Wheel location & 3 & 3 & 3 & 3 \\
\hline
\end{tabular}

\begin{tabular}{|l|c|c|c|c|}
\cline { 2 - 5 } \multicolumn{1}{c|}{} & \multicolumn{4}{c|}{ Structure 2 } \\
\hline Parameters & IRI & Rutting & Cracking & Overall \\
\hline Two-way AADT & 1 & 1 & 1 & 1 \\
\hline Tracffic Growth & 1 & 1 & 1 & 1 \\
\hline Dual tire spacing & 1 & 1 & 1 & 1 \\
\hline$\%$ Truck Design Direction & 1 & 1 & 1 & 1 \\
\hline$\%$ Heaw Vehicles & 1 & 1 & 1 & 1 \\
\hline Traffic Wander & 1 & 1 & 1 & 1 \\
\hline$\%$ Truck Design lane & 2 & 1 & 2 & 1 \\
\hline Operational Speed & 3 & 3 & 3 & 3 \\
\hline Average axle width & 3 & 3 & 3 & 3 \\
\hline Mean Wheel location & 3 & 3 & 3 & 3 \\
\hline
\end{tabular}




\section{CHAPTER 7: SENSITIVITY OF MEPDG TO MATERIAL PROPERTIES}

This chapter presents the sensitivity analysis of MEPDG to material properties parameters. The MEPDG sensitivity to material properties parameters is analyzed for structure 1 and 2, Figures 5.2 and 5.3 respectively. The steps in this study are:

1. Analysis of materials inputs to choose the parameters that are included in the study (Included in Appendix A).

2. Determination of the ranges of input values for each parameter (input space).

3. Latin Hypercube Sampling (LHS) is used to sample the entire input space of all input parameters in the analysis.

4. Every run in the LHS was used as input in the MEPDG.

5. The IRI, rutting, and cracking outputs from MEPDG were analyzed using multiple regressions with standardized regression coefficients (SRC) in order to categorize the relative importance among input parameters.

\subsection{Material Properties Parameters in the Analysis}

Input parameters and ranges include in the study are shown in Table 7.1 and Table 7.2 for structures 1 and 2 respectively. $\mathrm{Li}$ with $\mathrm{i}=1,2,3,4,5$ at the front of the name of each material properties indicates the layer in the pavement structure. For example, in the case of structure 1, L1 refers to layer 1 which is the top layer and L5 refers to layer 5 (subgrade). 
TABLE 7.1 Input Parameters and Ranges for Structure 1

\begin{tabular}{|c|c|c|c|}
\hline LAYER & PARAMETER & UNITS & RANGE \\
\hline L1 & Surface short-wave absorptive & & $0.8-0.9$ \\
\hline \multirow{6}{*}{ L1 } & L1 Effective binder content & $\%$ & $4.5-6.5$ \\
\hline & L1 Air Voids & $\%$ & $3-10$ \\
\hline & L1 Total unit weight & pcf & $145-150$ \\
\hline & L1 Poisson's ratio & \begin{tabular}{|r|}
- \\
\end{tabular} & $0.25-0.4$ \\
\hline & L1 Thermal conductivity asphalt & BTU/hr-ft-F & $0.5-0.8$ \\
\hline & L1 Heat capacity asphalt & BTU/lb-F ${ }^{o}$ & $0.22-0.5$ \\
\hline \multirow{6}{*}{$\mathbf{L} 2$} & L2 Effective binder content & $\%$ & $3.5-6$ \\
\hline & L2 Air voids & $\%$ & $3-10$ \\
\hline & L2 Total unit weight & pcf & $145-150$ \\
\hline & L2 Poisson's ratio & - & $0.25-0.4$ \\
\hline & L2 Thermal conductivity asphalt & BTU/hr-ft-F ${ }^{\circ}$ & $0.5-0.8$ \\
\hline & L2 Heat capacity asphalt & $\mathrm{BTU} / \mathrm{lb}-\mathrm{F}^{\mathrm{O}}$ & $0.22-0.50$ \\
\hline \multirow{6}{*}{$\mathbf{L 3}$} & L3 Effective binder content & $\%$ & $2.5-4.0$ \\
\hline & L3 Air voids & $\%$ & $6-12$ \\
\hline & L3 Total unit weight (pcf) & pcf & $145-150$ \\
\hline & L3 Poisson's ratio & \begin{tabular}{|l|}
- \\
\end{tabular} & $0.25-0.4$ \\
\hline & L3 Thermal conductivity asphalt & BTU/hr-ft-F ${ }^{\mathrm{o}}$ & $0.5-0.8$ \\
\hline & L3 Heat capacity asphalt & $\mathrm{BTU} / \mathrm{lb}-\mathrm{F}^{\mathrm{o}}$ & $0.22-0.5$ \\
\hline \multirow{6}{*}{ L4 } & L4 Effective binder content & $\%$ & $2-3$ \\
\hline & L4 Air voids & $\%$ & $15-20$ \\
\hline & L4 Total unit weight & pcf & $145-150$ \\
\hline & L4 Poisson's ratio & - & $0.25-0.4$ \\
\hline & L4 Thermal conductivity asphalt & BTU/hr-ft-F & $0.5-0.8$ \\
\hline & L4 Heat capacity asphalt & $\mathrm{BTU} / \mathrm{lb}-\mathrm{F}^{\mathrm{O}}$ & $0.22-0.50$ \\
\hline \multirow{5}{*}{ L5 } & L5 Poisson's ratio & - & $0.3-0.4$ \\
\hline & L5 Coefficient of lateral pressure Ko & - & $0.5-0.7$ \\
\hline & L5 Modulus & psi & $5000-9000$ \\
\hline & Tensile strength & psi & $500-1500$ \\
\hline & Mix coefficient of thermal contraction & $\mathrm{in} / \mathrm{in} /{ }^{\circ} \mathrm{F}$ & $2.2 \mathrm{E}-5-3.4 \mathrm{E}-5$ \\
\hline
\end{tabular}


TABLE 7.2 Input Parameters and Ranges for Structure 2

\begin{tabular}{|c|c|c|c|}
\hline LAYER & PARAMETER & UNITS & RANGE \\
\hline & Surface short-wave absorptive & & $0.8-0.9$ \\
\hline \multirow{6}{*}{$\mathbf{L 1}$} & L1 Effective binder content & $\%$ & $4.5-6.5$ \\
\hline & L1 Air voids & $\%$ & $3-10$ \\
\hline & L1 Total unit weight & pcf & $145-150$ \\
\hline & L1 Poisson's ratio & - & $0.25-0.4$ \\
\hline & L1 Thermal conductivity asphalt & $\mathrm{BTU} / \mathrm{hr}-\mathrm{ft}-\mathrm{F}^{\mathrm{o}}$ & $0.5-0.8$ \\
\hline & L1 Heat capacity asphalt & $\mathrm{BTU} / \mathrm{lb}-\mathrm{F}^{\mathrm{O}}$ & $0.22-0.5$ \\
\hline \multirow{3}{*}{$\mathbf{L 2}$} & L2 Poisson's ratio & - & $0.15-0.25$ \\
\hline & L2 Coefficient of lateral pressure (Ko) & - & $0.5-0.6$ \\
\hline & L2 Modulus & psi & $25000-35000$ \\
\hline \multirow{3}{*}{$\mathbf{L 3}$} & L3 Poisson's ratio & - & $0.15-0.25$ \\
\hline & L3 Coefficient of lateral pressure (Ko) & - & $0.5-0.6$ \\
\hline & L3 Modulus & psi & $15000-25000$ \\
\hline \multirow{5}{*}{ L4 } & L4 Poisson's ratio & - & $0.3-0.4$ \\
\hline & L4 Coefficient of lateral pressure (Ko) & - & $0.5-0.7$ \\
\hline & L4 Modulus & psi & $5000-9000$ \\
\hline & Average tensile strength at $14 \mathrm{oF}$ & psi & $500-1500$ \\
\hline & Mix coefficient of thermal contraction & $\mathrm{in} / \mathrm{in} /{ }^{\circ} \mathrm{F}$ & $2.2 \mathrm{E}-5-3.4 \mathrm{E}-5$ \\
\hline
\end{tabular}

\subsection{Latin Hypercube Sampling for Material Sensitivity Analysis}

A LHS for each structure was performed over the input parameters and ranges in Table 7.1 and 7.2. A different LHS is needed for each structure because the different layers require different material input parameters. The number of runs was defined as 10 times the number of parameters. 300 runs were defined for structure 1 and 200 runs were defined for structure 2. The LHS for each structure are shown in Appendix B. Multivariate analysis, as explained in section 6.1 and 6.2, was performed to evaluate relationships between individual pairs of input parameters in each LHS. Pairwise correlation coefficients for each pair of input parameters did not show relationships among individual parameters for either of the two LHS. For each structure, the LHS was used as inputs to run MEPDG and the sensitivity of input parameters on IRI, rutting, and cracking were analyzed using multiple regression analysis with SRC. 


\subsection{Results of Material Sensitivity on Structure 1}

The 300 runs defined in the LHS for structure 1 were input in MEPDG and run using the batch mode. Six batches of 50 runs each were performed. Using a computer with core i7 processor, $2.8 \mathrm{GHz}$, and $4 \mathrm{~GB}$ RAM in a 32-bit operating system the average running time was 15 minutes for a single run. 12.5 hours of continuous computer work were required for each batch, a total of 75 hours of computer processing for structure 1.

\subsubsection{IRI Sensitivity to Material Input Parameters on Structure 1}

A multiple regression model was performed to fit the material input parameters in the LHS and IRI output from MEPDG. The regression coefficients are indicators of the importance of the parameters and the magnitude of the SRC is an indicator of the relative importance among input parameters.

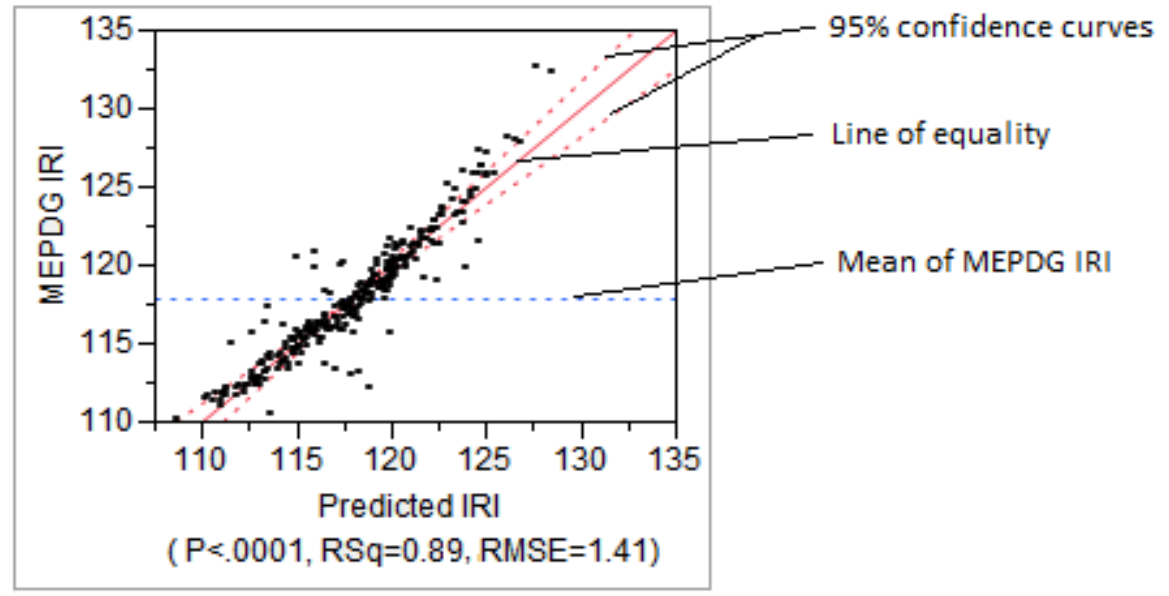

FIGURE 7.1 IRI Actual by Predicted Plot for Structure 1

Figure 7.1 displays the IRI output from MEPDG compared to the IRI predicted from the regression model. The line of equality (LOE) shows where the output from MEPDG and the predicted IRI are equal. The vertical distance from a point to the LOE is the difference between MEPDG output and the predicted IRI from the regression model. The horizontal dashed line represents the MEPDG prediction mean. The distance from a point to this horizontal line represents the residual without any effect in the model. The two slanted-dashed lines represent $95 \%$ confidence curves. The regression model is significant, P-value $<0.001$. The multiple 
regression coefficient is $\mathrm{R}^{2}=0.89$ and the standard deviation is small compared to the magnitude of IRI values, RMSE $=1.41$. These regression parameters indicate much of the variation of MEPDG IRI is explained by the regression model.

TABLE 7.3 IRI Standardized Regression Coefficients for Structure 1

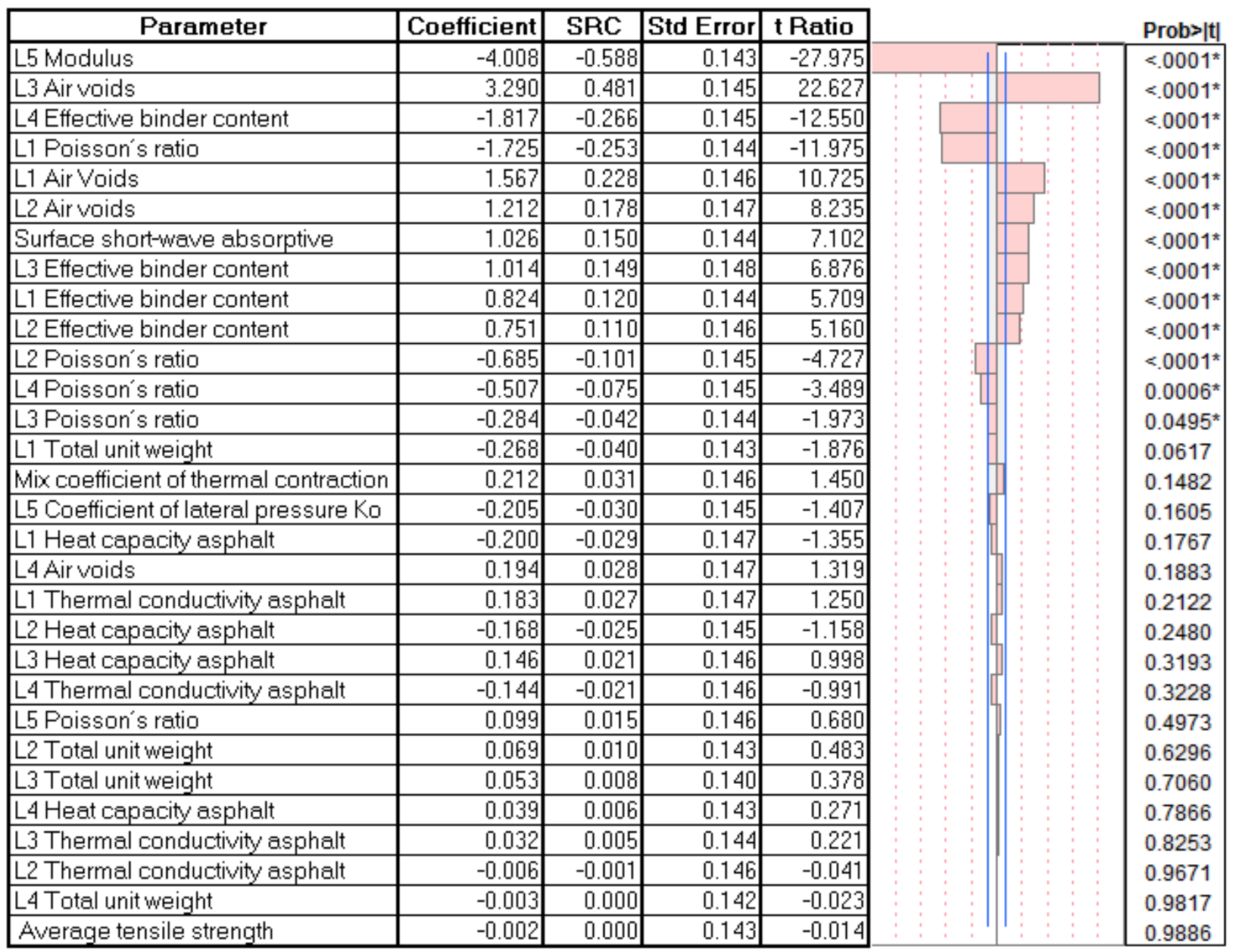

Table 7.3 shows the IRI regression coefficients, the SRC, standard errors, and t ratios for all input parameters. The input parameters have been sorted from highest to lowest according to the absolute value of the SRC. Some regression coefficients have positive values indicating that as the parameter increases the predicted IRI increases. Other parameters have negative regression coefficients indicating that as the parameter increases the predicted IRI decreases. The right column in the Table 7.3 indicates P-values which are the lowest level of significance that would lead to the rejection of the null hypothesis $\left(\mathrm{Ho}: \beta_{\mathrm{j}}=0\right)$. The significant parameters at significance level of 0.05 are identified with star in the table. 
The highest effects in IRI are due to resilient modulus of the subgrade, air voids of layer L3, and effective binder content of layer L4. Resilient modulus of subgrade, layer L5, was significant with a negative SRC for IRI. It is not difficult to suspect that the controlling distress here is rutting. Effective binder content was significant for all asphalt layers L1, L2, L3 and L4. Effective binder content has positive SRC for layers L1, L2, and L3 and negative SRC for L4. Layer L4 is the last asphalt layer in Structure 1 and it is on top of the subgrade. Tensile strains and stresses at the bottom of this layer are critical for bottom-up fatigue cracking. The negative SRC of L4 is consistent with this theory. Increasing the asphalt content in this layer would reduce bottom-up fatigue cracking but this might make the layer more susceptible to rutting. Equation 2.23 shows that IRI depends on cracking and rutting. So, variations in IRI when increasing effective binder content in any asphalt layer is expected because it affects rutting and cracking which are discussed in the following sections.

Poisson's ratio was significant for all asphalt layers, L1, L2, L3, and L4, with negative SRC. This is an interesting result because Poisson's ratio has been believed to have little effect. Poisson's ratio was not significant for subgrade, L5. Air voids as built was significant for layers L1, L2, and L3 but was not significant for layer L4. Air voids has positive SRC for all asphalt layers. So, an increase in air voids in any mix would lead to increase in IRI MEPDG predictions. Surface short-wave absorptive was significant with positive SRC. The others parameters in the study do not significantly affect IRI.

\subsubsection{Rutting Sensitivity to Material Input Parameters on Structure 1}

A multiple regression model among the material input parameters in the LHS and rutting output from MEPDG was performed. Figure 7.2 compares the rutting output from MEPDG with the rutting predicted from the regression model. The regression is significant, P-value $<0.001$.

The multiple regression coefficient, $\mathrm{R}^{2}=0.91$, and the standard deviation, $\mathrm{RMSE}=0.014$ indicate that the much of variation of MEPDG rutting is explained by the regression model. 


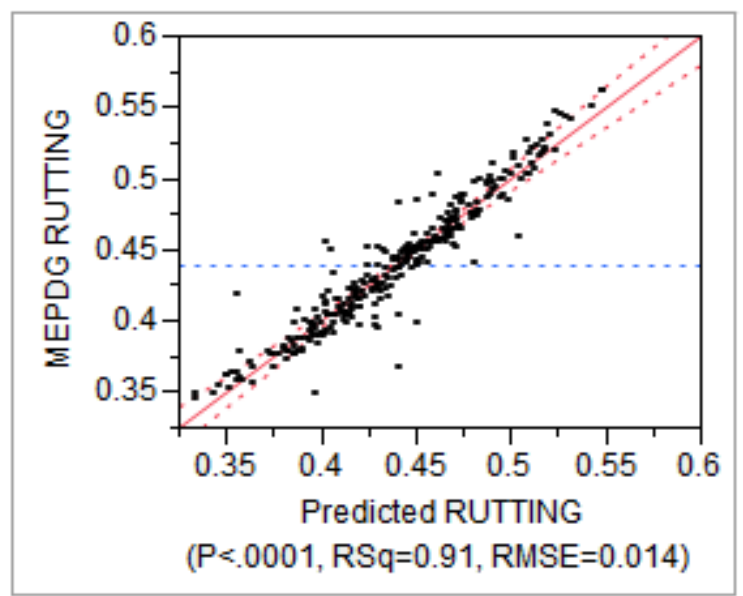

FIGURE 7.2 Rutting Actual by Predicted Plot for Structure 1

Total rutting in MEPDG is the cumulative permanent vertical deformation of all layers. In the past, the vertical deformation of subgrade played an important role in controlling rutting in mechanistic pavements design methods. MEPDG computes vertical permanent deformation for all layers.

Table 7.4 shows the rutting regression coefficients, SRC, standard errors, and t ratios for all input parameters. The highest effects in rutting are due to resilient modulus of the subgrade (L5), Poisson's ratio of layer L1, and as-built air voids of layers L1 and L2. Resilient modulus of subgrade has a negative SRC, then as resilient modulus of subgrade increases, MEPDG predicted rutting decreases. Coefficient of lateral pressure and Poisson's ratio of subgrade were not significant. Effective binder content was significant for layers L1 and L2 with positive SRC. Effective binder content was not significant for other layers. Asphalt heat capacity was significant for layers L1 and L2 with negative SRC. The deeper the layer the less significant is the effect of asphalt heat capacity. Poisson's ratio was significant with negative SRC for layer L1. This is interesting because Poisson's ratio was believed to have no major effect on predicted rutting. So, more care is needed in the determination of Poisson's ratio in asphalt layers. As-built air voids was significant with positive SRC for layers L1 and L2. As-built air voids was not significant for layers L3 and L4. Surface short-wave absorptive was significant with positive SRC. Thermal conductivity of asphalt was only significant for layer L1 with positive SRC. It can be seen that what makes a material property significant is not only the property itself, but also the 
depth of the layer in the pavement structure. The other parameters in the study do not significantly affect the predicted rutting from MEPDG.

TABLE 7.4 Rutting Standardized Regression Coefficients for Structure 1

\begin{tabular}{|c|c|c|c|c|c|}
\hline Parameter & \begin{tabular}{|l|} 
Coefficient \\
\end{tabular} & SRC & Std Error & t Ratio & Prob $>|t|$ \\
\hline L5 Modulus & -0.050 & -0.640 & 0.001 & -34.278 & $<.0001^{*}$ \\
\hline L1 Poisson's ratio & -0.034 & -0.431 & 0.001 & -23.034 & $<.0001^{\star}$ \\
\hline L1 Air Voids & 0.027 & 0.341 & 0.001 & 18.364 & $<.0001^{*}$ \\
\hline L2 Air voids & 0.022 & 0.281 & 0.002 & 14.649 & $<.0001^{*}$ \\
\hline Surface short-wave absorptive & 0.015 & 0.195 & 0.001 & 10.430 & $<.0001^{*}$ \\
\hline L2 Effective binder content & 0.013 & 0.168 & 0.001 & 8.903 & $<.0001^{*}$ \\
\hline L1 Effective binder content & 0.013 & 0.161 & 0.001 & 8.587 & $<.0001^{*}$ \\
\hline L2 Poisson's ratio & -0.011 & -0.138 & 0.001 & -7.283 & $<.0001^{*}$ \\
\hline L1 Heat capacity asphalt & -0.007 & -0.090 & 0.002 & -4.693 & $<.0001^{*}$ \\
\hline L2 Heat capacity asphalt & -0.005 & -0.065 & 0.001 & -3.451 & $0.0006^{*}$ \\
\hline L1 Thermal conductivity asphalt & 0.005 & 0.068 & 0.001 & 3.444 & $0.0007^{\star}$ \\
\hline L3 Air voids & 0.002 & 0.030 & 0.001 & 1.563 & 0.1192 \\
\hline L4 Effective binder content & 0.002 & 0.023 & 0.001 & 1.211 & 0.2270 \\
\hline L2 Total unit weight & -0.002 & -0.022 & 0.001 & -1.135 & 0.2573 \\
\hline L5 Poisson's ratio & -0.002 & -0.021 & 0.001 & -1.113 & 0.2667 \\
\hline L4 Total unit weight & 0.002 & 0.020 & 0.001 & 1.083 & 0.2798 \\
\hline L4 Air voids & 0.001 & 0.018 & 0.002 & 0.940 & 0.3483 \\
\hline L1 Total unit weight & -0.001 & -0.018 & 0.001 & -0.820 & 0.4131 \\
\hline L4 Thermal conductivity asphalt & -0.001 & -0.012 & 0.001 & -0.757 & 0.4496 \\
\hline Average tensile strength & 0.001 & 0.013 & 0.001 & 0.715 & 0.4751 \\
\hline Mix coefficient of thermal contraction & 0.001 & 0.011 & 0.001 & 0.599 & 0.5498 \\
\hline L3 Poisson's ratio & 0.001 & 0.008 & 0.001 & 0.432 & 0.6660 \\
\hline L3 Heat capacity asphalt & 0.001 & 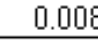 & 0.001 & 0.423 & 0.6727 \\
\hline L3 Thermal conductivity asphalt & -0.001 & -0.007 & 0.001 & -0.377 & 0.7064 \\
\hline L4 Poisson's ratio & 0.000 & 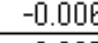 & 0.001 & -0.322 & 0.7474 \\
\hline L3 Total unit weight & 0.000 & 0.005 & 0.001 & 0.259 & 0.7961 \\
\hline L4 Heat capacity asphalt & 0.000 & 0.005 & 0.001 & 0.258 & 0.7966 \\
\hline L5 Coefficient of lateral pressure Ko & 0.000 & 0.002 & 0.001 & 0.120 & 0.9046 \\
\hline L3 Effective binder content & 0.000 & 0.001 & 0.002 & 0.051 & 0.9594 \\
\hline L2 Thermal conductivity asphalt & 0.000 & 0.000 & 0.001 & 0.008 & 0.9932 \\
\hline
\end{tabular}

\subsubsection{Cracking Sensitivity to Material Input Parameters on Structure 1}

A multiple regression model among the material input parameters in the LHS and cracking output from MEPDG was performed.

Figure 7.3 compares the cracking output from MEPDG with the cracking predicted from the regression model. The regression is significant, P-value $<0.001$ and the multiple regression coefficient, $\mathrm{R}^{2}=0.88$, and the standard deviation, $\mathrm{RMSE}=1.72$, indicate the variation on MEPDG cracking is well explained by the regression model. 


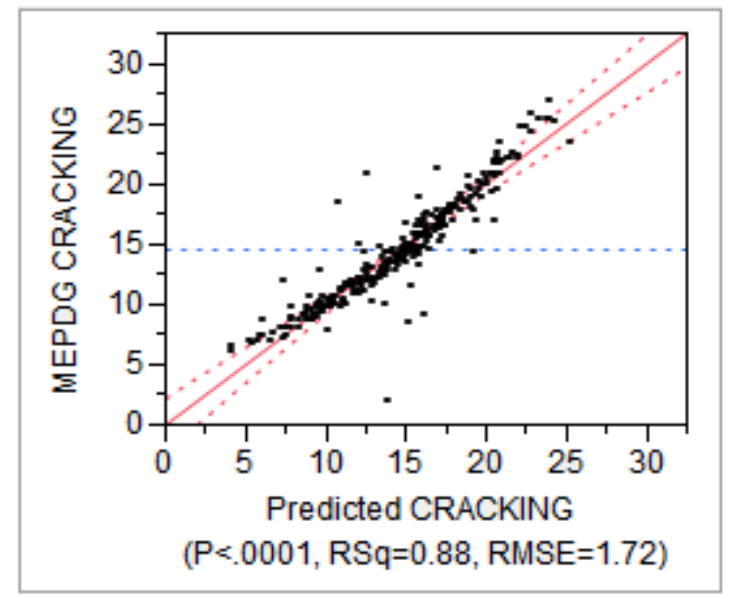

FIGURE7.3 Cracking Actual by Predicted Plot for Structure 1

Bottom up fatigue cracking starts at the bottom of the asphalt layer and propagates upwards. Surface-down fatigue cracking starts at the surface and propagates downwards. It is believed that most of the fatigue cracking occurs upwards. It was expected that parameters related with the stiffness of the lower asphalt layers and parameters related with the stiffness of the top layer to be significant. This is confirmed by the result of the sensitivity analysis displayed in Table 7.5.

The highest effects in cracking are due to as-built air voids of layer L3, resilient modulus of subgrade (layer L5), and effective binder content of layers L3 and L4. Effective binder content in layer L4 was significant parameter with negative SRC. An increase in asphalt content in L4 would lead to decrease in cracking predicted by MEPDG but this layer is an asphalt treated permeable base with low asphalt content and high as-built air voids. Effective binder layers L1 and L3 were significant with positive SRC. An increase in effective binder would lead to a decrease in stiffness. It is reasonable to think that a decrease in the stiffness in L3 would allows more bending effect in layer L4 increasing cracking as a result. As-built air voids was significant for layer L1, L2 and L3 with a positive SRC. The higher the air voids in these layers, the higher the MEPDG predicted cracking. Resilient modulus of subgrade (L5) was significant with negative SRC. Subgrade is related with deflections of the pavement structure that have significant effect in fatigue cracking of the asphalt layer. Poisson's ratio was significant for all asphalt layers, L1, L2, L3 and L4. Surface short-wave absorptive was also significant. The other parameters in the study do not significantly affect predicted cracking from MEPDG. 
TABLE 7.5 Cracking Standardized Regression Coefficients for Structure 1

\begin{tabular}{|c|c|c|c|c|c|}
\hline Parameter & Coefficient & SRC & Std Error & t Ratio & Prob $>|t|$ \\
\hline L3 Air voids & 5.514 & 0.682 & 0.178 & 31.005 & $<.0001^{\star}$ \\
\hline L5 Modulus & -3.434 & -0.426 & 0.175 & -19.594 & $<.0001^{*}$ \\
\hline L4 Effective binder content & -3.205 & -0.398 & 0.177 & -18.098 & $<.0001^{\star}$ \\
\hline L3 Effective binder content & 1.737 & 0.216 & 0.180 & 9.624 & $<.0001^{*}$ \\
\hline L4 Poisson's ratio & -0.830 & -0.103 & 0.178 & -4.671 & $<.0001^{*}$ \\
\hline L1 Air Voids & 0.786 & 0.097 & 0.179 & 4.399 & $<.0001^{*}$ \\
\hline Surface short-wave absorptive & 0.724 & 0.089 & 0.177 & 4.098 & $<.0001^{*}$ \\
\hline L1 Poisson's ratio & -0.648 & -0.080 & 0.176 & -3.679 & $0.0003^{*}$ \\
\hline L3 Poisson's ratio & -0.567 & -0.070 & 0.176 & -3.219 & $0.0014^{\star}$ \\
\hline L2 Air voids & 0.569 & 0.071 & 0.180 & 3.159 & $0.0018^{*}$ \\
\hline L1 Effective binder content & 0.525 & 0.065 & 0.177 & 2.973 & $0.0032^{*}$ \\
\hline L1 Total unit weight & -0.357 & -0.045 & 0.174 & -2.049 & $0.0415^{\star}$ \\
\hline L2 Poisson's ratio & -0.363 & -0.045 & 0.177 & -2.048 & $0.0416^{*}$ \\
\hline L2 Effective binder content & 0.329 & 0.041 & 0.178 & 1.846 & 0.0660 \\
\hline L5 Coefficient of lateral pressure Ko & -0.314 & -0.039 & 0.178 & -1.765 & 0.0787 \\
\hline L5 Poisson's ratio & 0.309 & 0.038 & 0.179 & 1.731 & 0.0846 \\
\hline Mix coefficient of thermal contraction & 0.263 & 0.032 & 0.179 & 1.471 & 0.1425 \\
\hline L2 Total unit weight & 0.232 & 0.030 & 0.175 & 1.326 & 0.1859 \\
\hline L3 Heat capacity asphalt & 0.181 & 0.022 & 0.179 & 1.011 & 0.3131 \\
\hline L4 Total unit weight & -0.146 & -0.019 & 0.173 & -0.843 & 0.4001 \\
\hline L4 Thermal conductivity asphalt & -0.146 & -0.018 & 0.178 & -0.821 & 0.4124 \\
\hline L4 Air voids & 0.136 & 0.017 & 0.180 & 0.756 & 0.4501 \\
\hline L1 Heat capacity asphalt & 0.126 & 0.016 & 0.180 & 0.699 & 0.4853 \\
\hline L3 Thermal conductivity asphalt & 0.075 & 0.009 & 0.177 & 0.423 & 0.6729 \\
\hline L3 Total unit weight & 0.056 & 0.007 & 0.171 & 0.329 & 0.7424 \\
\hline L2 Heat capacity asphalt & 0.046 & 0.006 & 0.178 & 0.257 & 0.7970 \\
\hline L1 Thermal conductivity asphalt & -0.045 & -0.006 & 0.179 & -0.253 & 0.8002 \\
\hline Average tensile strength & -0.040 & -0.005 & 0.175 & -0.230 & 0.8181 \\
\hline L4 Heat capacity asphalt & 0.018 & 0.002 & 0.175 & 0.104 & 0.9169 \\
\hline L2 Thermal conductivity asphalt & -0.009 & -0.001 & 0.178 & -0.048 & 0.9618 \\
\hline
\end{tabular}

\subsection{Results of Material Sensitivity on Structure 2}

The 200 runs defined in the LHS for structure 2 were input in MEPDG and run using the batch mode. Four batches of 50 runs each were performed. Using a computer with core i7 processor, $2.8 \mathrm{GHz}$, and $4 \mathrm{~GB}$ RAM in a 32-bit operating system the average running time was 15 minutes for a single run. 12.5 hours of continuous computer work were required for each batch, a total of 50 hours of computer processing for structure 2 .

\subsubsection{IRI Sensitivity to Material Input Parameters on Structure 2}

A multiple regression model among the material input values in the LHS and IRI output from MEPDG was performed. 


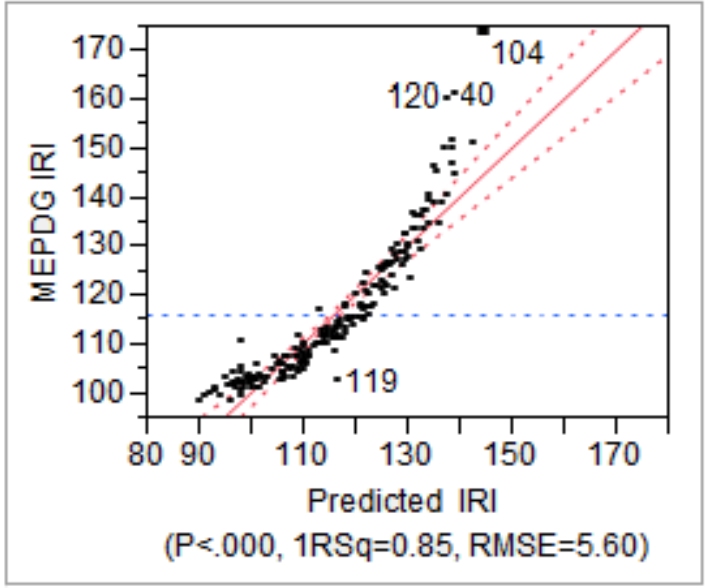

(a)

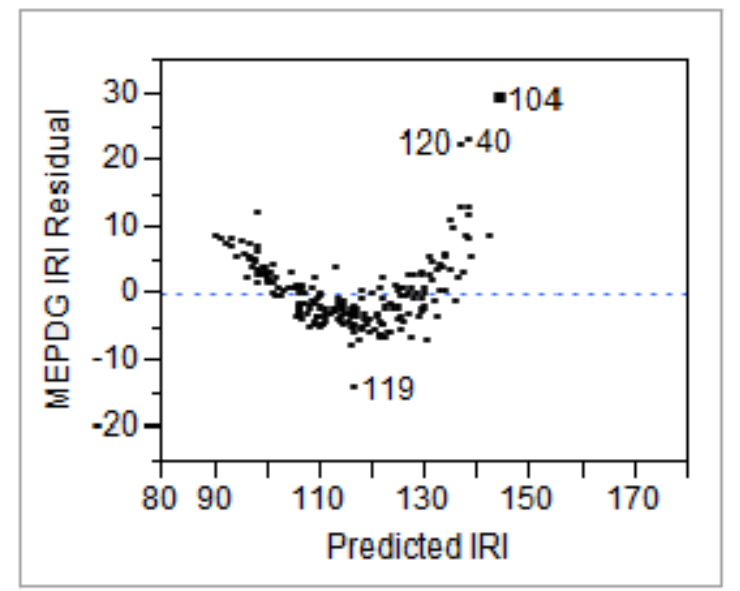

(b)

FIGURE 7.4 IRI Actual by Predicted Plot for Structure 2

Figure 7.4 (a) compares the IRI output from MEPDG with the IRI predicted from the regression model for structure 2. Although, the regression is significant, P-value $<0.001$, runs 119, 120, 40, and 104 seem to be outliers. The regression model does not do a good job of predicting these runs. Additionally, non-linear trend is suspected from the curvature in the figure. The residual plot in Figure 7.4 (b) shows curvature of the residuals which confirms the nonlinear trend of IRI. Figure 7.4 (b) also confirms that points 119, 120, 40, and 104 are outliers. The analysis for rutting and cracking also showed that these points are outliers; therefore they were removed from the data set. Rank transformation was performed to remove non-linearity from the analysis.

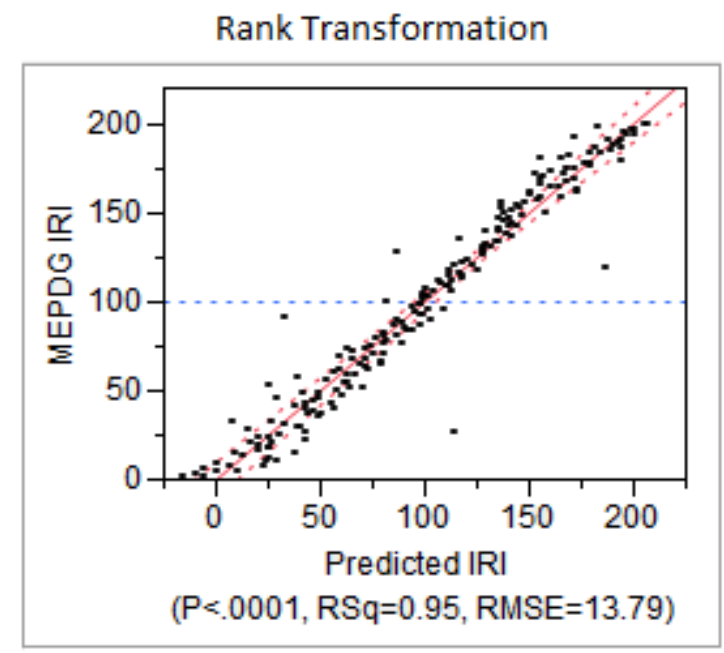

FIGURE7.5 IRI Actual by Predicted Plot for Structure 2 
Figure 7.5 compares rank-transformed IRI from MEPDG with rank-transformed IRI predicted from the regression model. The scale of axes in the figure changed because the transformation replaces the inputs and outputs for their ranks. With 200 runs the transformed scale is from 1 to 200 . The regression is significant, $\mathrm{P}$-value $<0.0001$, and the multiple regression coefficient, $\mathrm{R}^{2}=0.95$, and the standard deviation, $\mathrm{RMSE}=13.79$, indicate that much of the variation of MEPDG IRI is explained by the regression model.

Table 7.6 shows that most sensitive parameters are as-built air voids and effective binder content of layer L1, and resilient modulus of layers L4 and L2. Resilient modulus was also significant for layers L3. As-built air voids of L1 have positive SRC, resilient modulus has negative SRC for all unbound layers, and effective binder content has negative SRC. Poisson's ratio was significant for layer L1 with negative SRC and layer L2 with positive SRC. L1 is an asphalt layer and L2 is an unbound granular base in structure 2. Surface short-wave absorptive was significant with positive SRC. Heat capacity of L1 was significant with negative SRC. The other parameters in the study were not significant for structure 2. L4 modulus, L2 modulus, and L3 modulus have large regression coefficients. Collinearity in the data may lead to these results but multivariate analysis did not reveal collinearity. Rank transformation with large number of runs may lead to large coefficients because the large difference in the input values. This is the reason why $\mathrm{SRC}$ is a betters approach.

TABLE 7.6 IRI Standardized Regression Coefficients for Structure 2

\begin{tabular}{|c|c|c|c|c|c|}
\hline Parameter & Coefficient & SRC & Std Error & t Ratio & Prob $>|t| t \mid$ \\
\hline L1 Air voids & 3.265 & 0.939 & \begin{tabular}{|r|}
0.048 \\
\end{tabular} & 68.420 & $<.0001^{*}$ \\
\hline L4 Modulus & -361.990 & -0.184 & 26.741 & -13.537 & $<.0001^{\star}$ \\
\hline L1 Effective binder content & -0.140 & -0.142 & 0.013 & -10.369 & $<.0001^{*}$ \\
\hline L2 Modulus & -695.017 & -0.141 & 68.044 & -10.214 & $<.0001^{\star}$ \\
\hline L1 Poisson's ratio & -0.005 & -0.068 & 0.001 & -5.035 & $<.0001^{\star}$ \\
\hline L3 Modulus & -333.490 & -0.068 & 67.638 & -4.930 & $<.0001^{\star}$ \\
\hline Surface short-wave absorptive & 0.002 & 0.048 & 0.001 & 3.546 & $0.0005^{\star}$ \\
\hline L2 Poisson's ratio & 0.002 & 0.038 & 0.001 & 2.750 & $0.0066^{\star}$ \\
\hline L1 Heat capacity asphalt & -0.004 & -0.029 & 0.002 & -2.146 & $0.0332^{*}$ \\
\hline L4 Coefficient of lateral pressure Ko & -0.002 & -0.021 & 0.001 & -1.559 & 0.1208 \\
\hline Average tensile strength at $140 \mathrm{~F}$ & 8.296 & 0.017 & 6.662 & 1.245 & 0.2147 \\
\hline L3 Coefficient of lateral pressure Ko & -0.001 & -0.016 & 0.001 & -1.146 & 0.2532 \\
\hline L1 Thermal conductivity asphalt & 0.002 & 0.014 & 0.002 & 1.004 & 0.3169 \\
\hline L4 Poisson's ratio & -0.001 & -0.011 & 0.001 & -0.834 & 0.4053 \\
\hline L1 Total unit weight & 0.028 & 0.011 & 0.034 & 0.824 & 0.4113 \\
\hline Mix coefficient of thermal contraction & 0.000 & 0.009 & 0.000 & 0.662 & 0.5088 \\
\hline L3 Poisson's ratio & 0.000 & 0.009 & 0.001 & 0.660 & 0.5101 \\
\hline L2 Coefficient of lateral pressure Ko & 0.000 & 0.000 & 0.001 & 0.025 & 0.9799 \\
\hline
\end{tabular}


IRI data were fitted to a Gaussian process, Figure 7.6, to verify the results of the rank transformation. The points in Figure 7.6 are close to the 45 degree diagonal line showing that the Gaussian process does a good job of predicting MEPDG IRI. Comparison between rank transformation and Gaussian process results shows that the same significant parameters are identified in both approaches. Among the highly significant parameters in Table 7.6, $\mathrm{P}<.0001$, only L4 modulus moved two places down under effective binder content of layer L1 and resilient modulus of layer L2. Notice that these two parameters have similar values of SRC in the rank transformation. Among the other significant parameters, surface short-wave absorptive and Poisson's ration of layer L2 swapped positions but their percent of effect in Figure 7.6 have same value. Heat capacity of layer L1 is the last significant parameter in Table 7.6 while having no effect in Figure 7.6. The not significant parameters have zero effect in the Gaussian process approach thus the order is not meaningful for these parameters. In general, the two approaches agree in the sensitivity of the input parameters.

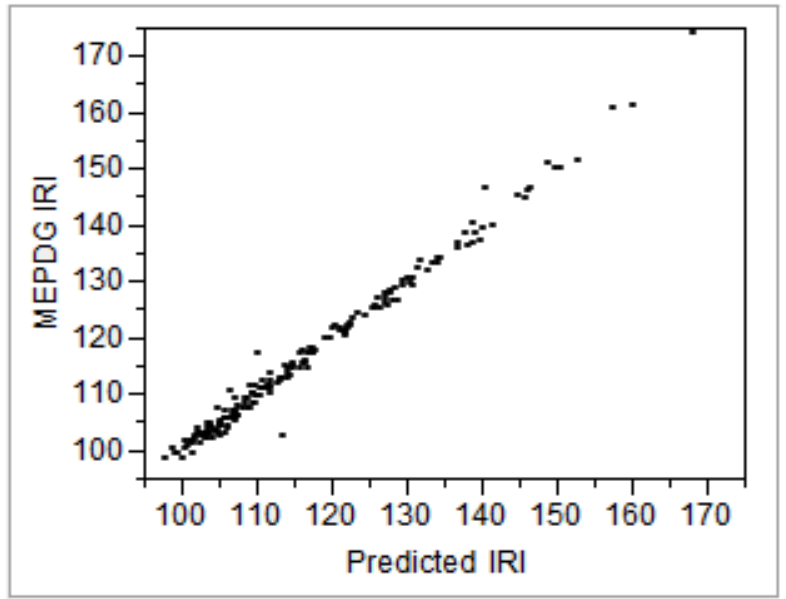

\begin{tabular}{|l|r|}
\hline \multicolumn{1}{|c|}{ Parameters } & Effect \\
\hline L1 Air voids & 0.834 \\
\hline L1 Effective binder content & 0.045 \\
\hline L2 Modulus & 0.026 \\
\hline L4 Modulus & 0.014 \\
\hline L1 Poisson's ratio & 0.005 \\
\hline L3 Modulus & 0.005 \\
\hline L2 Poisson's ratio & 0.001 \\
\hline Surface short-wave absorptive & 0.001 \\
\hline L3 Poisson's ratio & 0.000 \\
\hline Average tensile strength at 14oF & 0.000 \\
\hline L1 Heat capacity asphalt & 0.000 \\
\hline L1 Thermal conductivity asphalt & 0.000 \\
\hline L2 Coefficient of lateral pressure Ko & 0.000 \\
\hline L1 Total unit weight & 0.000 \\
\hline L4 Coefficient of lateral pressure Ko & 0.000 \\
\hline L3 Coefficient of lateral pressure Ko & 0.000 \\
\hline L4 Poisson's ratio & 0.000 \\
\hline Mix coefficient of thermal contraction & 0.000 \\
\hline
\end{tabular}

FIGURE 7.6 Verification of IRI Sensitivity with a Gaussian Process

\subsubsection{Rutting Sensitivity to Material Input Parameters on Structure 2}

A multiple regression model was performed to fit the material input values in the LHS and the rutting output from MEPDG. Figure 7.7 compares the rutting output from MEPDG versus the rutting predicted with the regression model for structure 2 . The regression is 
significant, $\mathrm{P}$-value $<0.0001$. The multiple regression coefficient, $\mathrm{R}^{2}=0.97$, and the standard deviation, $\mathrm{RMSE}=0.0098$ indicate that much of the variation of MEPDG rutting is explained by the regression model.

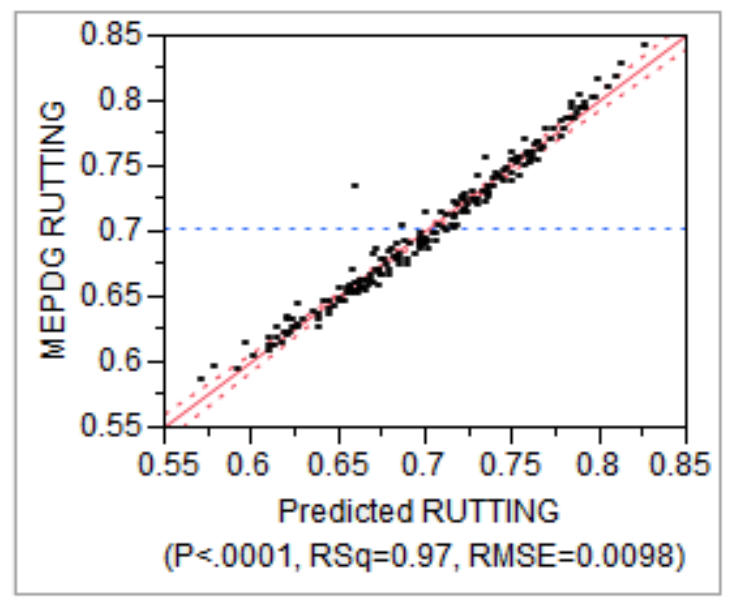

FIGURE 7.7 Rutting Actual by Predicted Plot for Structure 2

Table 7.7 shows that resilient modulus of layers L4, L3, and L2, and as-built air voids and effective binder content of layer L1 are the most significant parameters. Resilient modulus of unbound layers has negative SRC. As-built air voids and effective binder content of layer L1 have positive SRC. An increase of these two parameters would increase predicted MEPDG rutting in structure 2 . The results confirm that the resilient modulus of the subgrade has a significant effect on rutting. Surface short-wave absorptive was significant with positive SRC. Heat capacity of layer L1 was significant with a negative SRC. An increase in the heat capacity of the asphalt means that for a given change in air temperature there is a smaller change in the temperature in the asphalt. As the ambient temperature rises, a material with a high heat capacity will not become as hot as a material with a low heat capacity. The viscosity drop due to temperature is therefore lower for the asphalt with a high heat capacity. Rutting potential of high viscosity asphalt is less for low viscosity asphalt. Hence a negative SRC for heat capacity is reasonable. Poisson's ratio was significant for layers L1, L2, L3 with negative SRC for L1 and positive SRC for L2 and L3. L1 is an asphalt layer and has negative SRC for Poisson's ratio and all unbound layers have positive SRC for Poisson's ratio. All other parameters are not significant for rutting. 
TABLE 7.7 Rutting Standardized Regression Coefficients for Structure 2

\begin{tabular}{|c|c|c|c|c|c|}
\hline Parameter & Coefficient & SRC & Std Error & t Ratio & Prob $>|t|$ \\
\hline L4 Modulus & -0.080 & -0.826 & 0.00122 & -64.954 & $<.0001^{\star}$ \\
\hline L1 Air voids & 0.035 & 0.357 & 0.00125 & 27.677 & $<.0001^{*}$ \\
\hline L3 Modulus & -0.025 & -0.263 & 0.00124 & -20.468 & $<.0001^{*}$ \\
\hline L2 Modulus & -0.017 & -0.172 & 0.00125 & -13.332 & $<.0001^{*}$ \\
\hline L1 Effective binder content & 0.015 & 0.154 & 0.00124 & 11.939 & $<.0001^{*}$ \\
\hline Surface short-wave absorptive & 0.009 & 0.089 & 0.00122 & 6.980 & $<.0001^{\star}$ \\
\hline L1 Poisson's ratio & -0.008 & -0.087 & 0.00121 & -6.882 & $<.0001^{*}$ \\
\hline L1 Heat capacity asphalt & -0.007 & -0.077 & 0.00122 & -6.017 & $<.0001^{*}$ \\
\hline L2 Poisson's ratio & 0.006 & 0.058 & 0.00123 & 4.507 & $<.0001^{*}$ \\
\hline L3 Poisson's ratio & 0.004 & 0.038 & 0.00123 & 2.962 & $0.0035^{*}$ \\
\hline L3 Coefficient of lateral pressure Ko & -0.001 & -0.014 & 0.00124 & -1.086 & 0.2787 \\
\hline L4 Coefficient of lateral pressure Ko & -0.001 & -0.012 & 0.00124 & -0.972 & 0.3326 \\
\hline L2 Coefficient of lateral pressure Ko & -0.001 & -0.008 & 0.00122 & -0.608 & 0.5441 \\
\hline L1 Total unit weight & -0.001 & -0.007 & 0.00120 & -0.559 & 0.5766 \\
\hline Mix coefficient of thermal contraction & 0.001 & 0.007 & 0.00122 & 0.512 & 0.6094 \\
\hline Average tensile strength at $140 \mathrm{~F}$ & 0.000 & 0.004 & 0.00122 & 0.308 & 0.7585 \\
\hline L4 Poisson's ratio & 0.000 & 0.004 & 0.00122 & 0.280 & 0.7800 \\
\hline L1 Thermal conductivity asphalt & 0.000 & -0.001 & 0.00124 & -0.072 & 0.9424 \\
\hline
\end{tabular}

\subsubsection{Cracking Sensitivity to Material Input Parameters on Structure 2}

A multiple regression model among the material input values in the LHS and rutting output from MEPDG was performed.

Figure 7.8 (a) displays the cracking output from MEPDG versus the cracking predicted from the regression model for structure 2. Although, the regression parameters indicate good fit, inspection of the figure reveals non-linear trend. Additionally, the regression model predicts negative cracking for values of MEPDG cracking smaller than 5\%. Figure 7.8(b) shows cracking output from MEPDG versus the cracking predicted from the regression model after dropping the cracking values smaller than 5\%. The regression improved $\left(\mathrm{R}^{2}=0.97\right)$ and all negative predictions from the regression model were removed. The sensitivity analysis was performed with this last approach. 


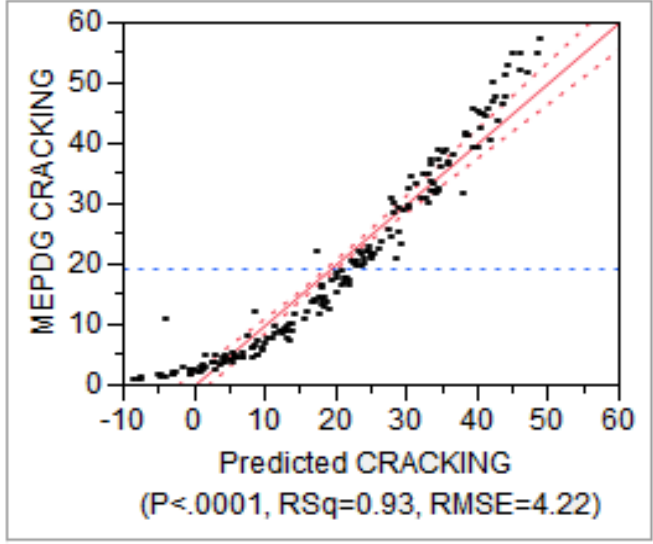

(a)

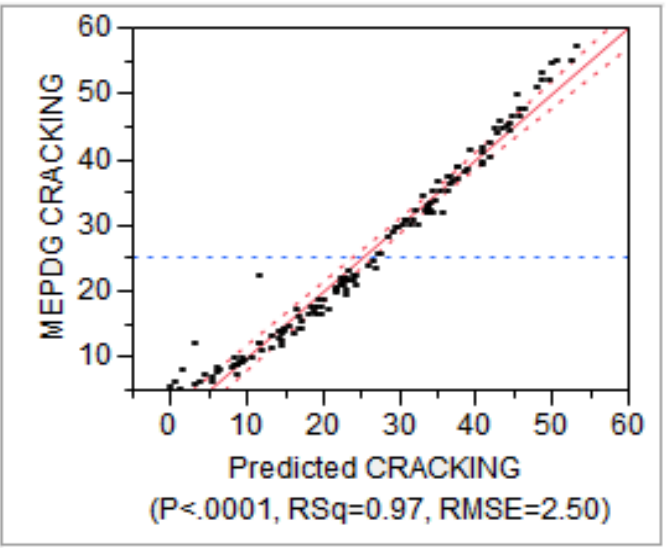

(b)

FIGURE 7.8 Cracking Actual by Predicted Plot for Structure 2

Table 7.8 shows the most significant parameters in structure 2 are as-built air voids, effective binder content of layer L1, and resilient modulus of layer 2. As-built air voids has positive SRC while effective binder content has negative SRC. It is clear that an increase in effective binder content would lead to a decrease in cracking on structure 2 but it may favor rutting. Resilient modulus was significant for layer L2 with negative SRC. Resilient modulus has negative SRC for all unbound granular layers. An increase in resilient modulus in unbound of the layers would lead to reduced MEPDG cracking predictions for structure 2. Poisson's ratio is significant in layer L1 with negative SRC. No others parameters are significant on MEPDG predicted cracking.

TABLE 7.8 Cracking Standardized Regression Coefficients for Structure 2

\begin{tabular}{|c|c|c|c|c|c|}
\hline Term & Coefficient & SRC & Std Error & t Ratio & Prob $>|t|$ \\
\hline L1 Air voids & 31.781 & 0.970 & 0.514 & 61.796 & $<.0001^{*}$ \\
\hline L1 Effective binder content & -8.118 & -0.339 & 0.380 & -21.380 & $<.0001^{\star}$ \\
\hline L2 Modulus & -4.062 & -0.163 & 0.388 & -10.473 & $<.0001^{*}$ \\
\hline L1 Poisson's ratio & -2.058 & -0.087 & 0.361 & -5.708 & $<.0001^{*}$ \\
\hline L2 Poisson's ratio & 1.741 & 0.072 & 0.368 & 4.734 & $<.0001^{*}$ \\
\hline L3 Modulus & -1.457 & -0.060 & 0.381 & -3.823 & $0.0002^{*}$ \\
\hline Surface short-wave absorptive & 0.545 & 0.022 & 0.374 & 1.458 & 0.1473 \\
\hline L1 Total unit weight & -0.493 & -0.021 & 0.365 & -1.351 & 0.1792 \\
\hline L1 Heat capacity asphalt & -0.473 & -0.020 & 0.359 & -1.319 & 0.1898 \\
\hline L4 Poisson's ratio & -0.416 & -0.018 & 0.368 & -1.129 & 0.2609 \\
\hline L3 Coefficient of lateral pressure Ko & 0.385 & 0.016 & 0.373 & 1.035 & 0.3029 \\
\hline L3 Poisson's ratio & -0.342 & -0.014 & 0.372 & -0.919 & 0.3597 \\
\hline Average tensile strength at $140 \mathrm{~F}$ & 0.303 & 0.013 & 0.370 & 0.818 & 0.4151 \\
\hline L4 Coefficient of lateral pressure Ko & -0.302 & -0.012 & 0.383 & -0.787 & 0.4328 \\
\hline L1 Thermal conductivity asphalt & 0.290 & 0.012 & 0.382 & 0.760 & 0.4487 \\
\hline L2 Coefficient of lateral pressure Ko & 0.189 & 0.008 & 0.370 & 0.510 & 0.6110 \\
\hline Mix coefficient of thermal contraction & 0.131 & 0.005 & 0.367 & 0.358 & 0.7209 \\
\hline L4 Modulus & -0.067 & -0.003 & 0.364 & -0.183 & 0.8549 \\
\hline
\end{tabular}




\subsection{Summary of Results of Material Inputs Sensitivity}

The sensitivity of MEPDG outputs to material properties of structure 1 is summarized in Table 7.9 and Figure 7.9. The bars represent the SRC and a star in front of a bar indicates significant effect at $95 \%$ of confidence level. Bars on the left side of the figure represent negative SRC while bars on the right side represent positive SRC. For all material properties, all significant effects maintain the same sign of SRC in the figure for all distresses. Consequently, any modification or change of significant material properties to improve predicted performance in MEPDG will have effect on all distresses.

The largest effects on IRI in structure 1 are due to resilient modulus of subgrade (layer L5), as-built air voids of layer L3, effective binder content of layer L4, and Poisson's ratio of layer L1. As-bulit air voids of layers L1 and L2, effective binder content of layer L1, L2 and L3, surface short-wave absorptive, Poisson's ratio of L2, L3 and L4 also have significant effect on predicted IRI from MEPDG. The other parameters in the study do not significantly affect predicted IRI from MEPDG. 13 of the 30 parameters in the study have significant effect on IRI. 6 of the 13 significant parameters have negative SRC while the other 7 significant parameters have positive SRC.

Resilient modulus of subgrade (L5), Poisson's ratio of layer L1, and as-built air voids of layers L1 and L2 have the largest effect on rutting predicted from MEPDG. Other significant effects on rutting for structure 1 are surface short-wave absorptive, effective binder content of L1 and L2, Poisson's ratio of L2, and heat capacity of L1 and L2. 11 of the 30 parameters in the study have a significant effect on rutting. 5 of the 11 significant parameters have negative SRC while the other 6 significant parameters have positive SRC.

The material properties with the largest effect on cracking on structure 1 are as-built air voids of L3, resilient modulus of subgrade (L5), and effective binder content of L3 and L4. Effective binder content of L1 was also significant along with as-built air voids of L1 and L2, surface short-wave absorptive, total unit weight of L1, and Poisson's ratio of L1, L2, L3 and L4. 13 of the 30 parameters in the study have a significant effect on cracking on structure 1.7 of the 13 significant parameters have negative SRC while the other 6 significant parameters have positive SRC. 
An increase in effective binder content of L4 within the input ranges would reduce MEPDG cracking and IRI without significant effects on rutting. Reduction of air void in L3 would reduce MEPDG cracking and IRI without significantly affecting rutting. Resilient modulus of subgrade is highly significant with negative SRC for all distresses. An increase in resilient modulus of subgrade would lead to better general performance of structure 1.

TABLE 7.9 Standardized Regression Coefficients of Structure 1

\begin{tabular}{|l|r|r|r|}
\cline { 2 - 4 } \multicolumn{1}{c|}{ Term } & \multicolumn{3}{c|}{ SRC } \\
\hline \multicolumn{1}{c|}{ IRI } & \multicolumn{1}{c|}{ Rutting } & Cracking \\
\hline L5 Modulus & -0.588 & -0.640 & -0.426 \\
\hline L3 Air voids & 0.481 & 0.030 & 0.682 \\
\hline L4 Effective binder content & -0.266 & 0.023 & -0.398 \\
\hline L1 Poisson's ratio & -0.253 & -0.431 & -0.080 \\
\hline L1 Air Voids & 0.228 & 0.346 & 0.097 \\
\hline L2 Air voids & 0.178 & 0.281 & 0.071 \\
\hline Surface short-wave absorptive & 0.150 & 0.195 & 0.089 \\
\hline L3 Effective binder content & 0.149 & 0.001 & 0.216 \\
\hline L1 Effective binder content & 0.120 & 0.161 & 0.065 \\
\hline L2 Effective binder content & 0.110 & 0.168 & 0.041 \\
\hline L2 Poisson's ratio & -0.101 & -0.138 & -0.045 \\
\hline L4 Poisson's ratio & -0.075 & -0.006 & -0.103 \\
\hline L3 Poisson's ratio & -0.042 & 0.008 & -0.070 \\
\hline L1 Total unit weight & -0.040 & -0.016 & -0.045 \\
\hline Mix coefficient of thermal contraction & 0.031 & 0.011 & 0.032 \\
\hline L5 Coefficient of lateral pressure Ko & -0.030 & 0.002 & -0.039 \\
\hline L1 Heat capacity asphalt & -0.029 & -0.090 & 0.016 \\
\hline L4 Air voids & 0.028 & 0.018 & 0.017 \\
\hline L1 Thermal conductivity asphalt & 0.027 & 0.066 & -0.006 \\
\hline L2 Heat capacity asphalt & -0.025 & -0.065 & 0.006 \\
\hline L3 Heat capacity asphalt & 0.021 & 0.008 & 0.022 \\
\hline L4 Thermal conductivity asphalt & -0.021 & -0.014 & -0.018 \\
\hline L5 Poisson's ratio & 0.015 & -0.021 & 0.038 \\
\hline L2 Total unit weight & 0.010 & -0.022 & 0.030 \\
\hline L3 Total unit weight & 0.008 & 0.005 & 0.007 \\
\hline L4 Heat capacity asphalt & 0.006 & 0.005 & 0.002 \\
\hline L3 Thermal conductivity asphalt & 0.005 & -0.007 & 0.009 \\
\hline L2 Thermal conductivity asphalt & -0.001 & 0.000 & -0.001 \\
\hline L4 Total unit weight & 0.000 & 0.020 & -0.019 \\
\hline Average tensile strength & 0.000 & 0.013 & -0.005 \\
\hline
\end{tabular}




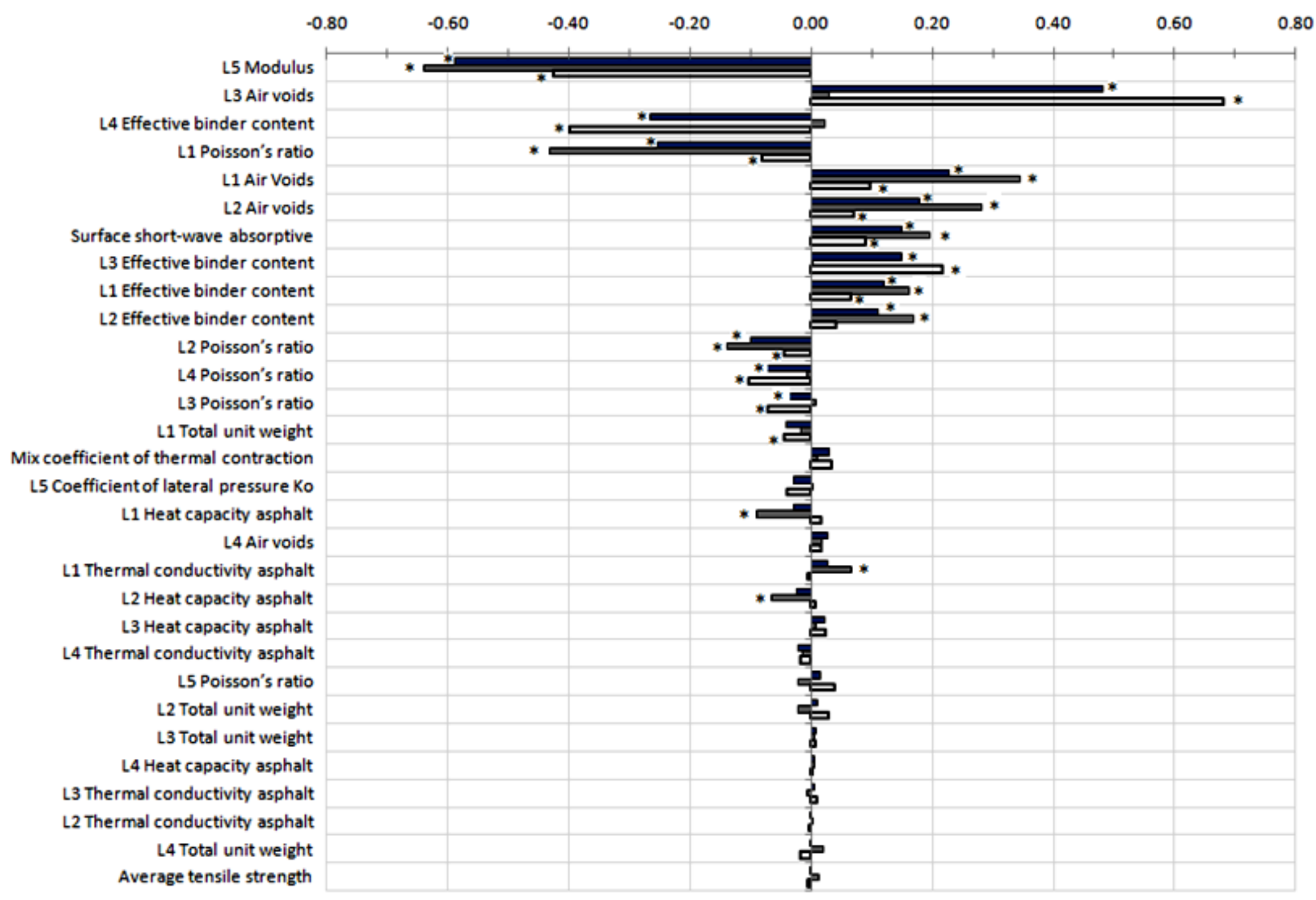

* Significant Effect $\square$ IRI $\square$ Rutting $\square$ Cracking

FIGURE 7.9 Standardized Regression Coefficients for Structure 1 
A summary of SRC for structure 2 is shown in Table 7.10 and Figure 7.10. The largest effect on IRI and cracking are due to air voids of layer L1. The largest effect on rutting is due to resilient modulus of subgrade (L4). As-built air voids of layer L1 is significant for all distresses with positive SRC. Effective binder content of L1 is significant for all distresses with negative SRC for IRI and cracking but positive for rutting. An increase in effective binder content of layer L1 would reduce MEPDG IRI and cracking but increase rutting prediction. Resilient modulus of L2, L3, and L4 have large effect on rutting. Resilient modulus of L2, and Poisson's ration of L1 are significant for all distresses. Out of 18 input parameters 9 are significant for IRI, 10 are significant for rutting, and 7 are significant for cracking in structure 2.

A direct comparison between structure 1 and 2 is not possible because they have different numbers of layers with different material properties. All layers in structure 1 are asphalt over a subgrade while structure 2 has two unbound layers in the middle of an asphalt layer and a subgrade. Even so, it is possible to compare the result for the surface asphalt layers and subgrade.

The sensitivity of material properties of subgrade, layer L5 in structure 1 and L4 in structure 2, show that resilient modulus have the same sign of SRC for both structures. The SRC of Poisson's ratio subgrade was not significant for all distresses on both structures. Coefficient of lateral pressure of subgrade was not significant for both structures with negative SRC for all distresses in structure 2. Correspondingly, coefficient of lateral pressure of subgrade has negative SRC for IRI and cracking while having a small positive SRC for rutting in structure 1. Coefficient of lateral pressure of subgrade has small negative SRC for all distresses in structure 2. Since the MEPDG output are fitted to a multiple linear regression model, the sign of very small values of SRC is not accurately predicted by the model but this is negligible because parameters with a very small SRC do not significantly affect the MEPDG outputs. Some differences for the significance of the parameters are found between the structures. Resilient modulus of subgrade is significant for all distresses in structure 1but only significant for rutting and IRI in structure 2. Since the analysis of significance of parameters is made with the MEPDG output using t-test, the magnitudes of those outputs play an important role in this analysis. In other words, the performance of the structure is a significant factor. This clearly identifies 
interaction between the type of structure and material input parameters. This was not the case in the traffic analysis where the results from the analysis are independent of the structure.

The comparison of the asphalt layers (L1) between both structures reveals that the sign of SRC are consistent except for effective binder content for IRI and cracking. As shown in equation 2.23, IRI depends on cracking, rutting, and site factor. Because site factor was constant in this study then cracking might be the main factor in this result.

TABLE 7.10 Standardized Regression Coefficients of Structure 2

\begin{tabular}{|l|r|r|r|}
\cline { 2 - 4 } \multicolumn{1}{c|}{} & \multicolumn{3}{c|}{ SRC } \\
\hline \multicolumn{1}{c|}{ Term } & \multicolumn{1}{c|}{ IRI } & \multicolumn{1}{c|}{ Rutting } & Cracking \\
\hline L1 Air voids & 0.939 & 0.357 & 0.970 \\
\hline L4 Modulus & -0.184 & -0.826 & -0.003 \\
\hline L1 Effective binder content & -0.142 & 0.154 & -0.339 \\
\hline L2 Modulus & -0.141 & -0.172 & -0.163 \\
\hline L1 Poisson's ratio & -0.068 & -0.087 & -0.087 \\
\hline L3 Modulus & -0.068 & -0.263 & -0.060 \\
\hline Surface short-wave absorptive & 0.048 & 0.089 & 0.022 \\
\hline L2 Poisson's ratio & 0.038 & 0.058 & 0.073 \\
\hline L1 Heat capacity asphalt & -0.029 & -0.077 & -0.020 \\
\hline L4 Coefficient of lateral pressure & -0.021 & -0.012 & -0.012 \\
\hline Average tensile strength at 14oF & 0.017 & 0.004 & 0.013 \\
\hline L3 Coefficient of lateral pressure Ko & -0.016 & -0.014 & 0.016 \\
\hline L1 Thermal conductivity asphalt & 0.014 & -0.001 & 0.012 \\
\hline L4 Poisson's ratio & -0.011 & 0.004 & -0.018 \\
\hline L1 Total unit weight & 0.011 & -0.007 & -0.021 \\
\hline L3 Poisson's ratio & 0.009 & 0.038 & -0.014 \\
\hline Mix coefficient of thermal contraction & 0.009 & 0.007 & 0.005 \\
\hline L2 Coefficient of lateral pressure Ko & 0.000 & -0.008 & 0.008 \\
\hline
\end{tabular}




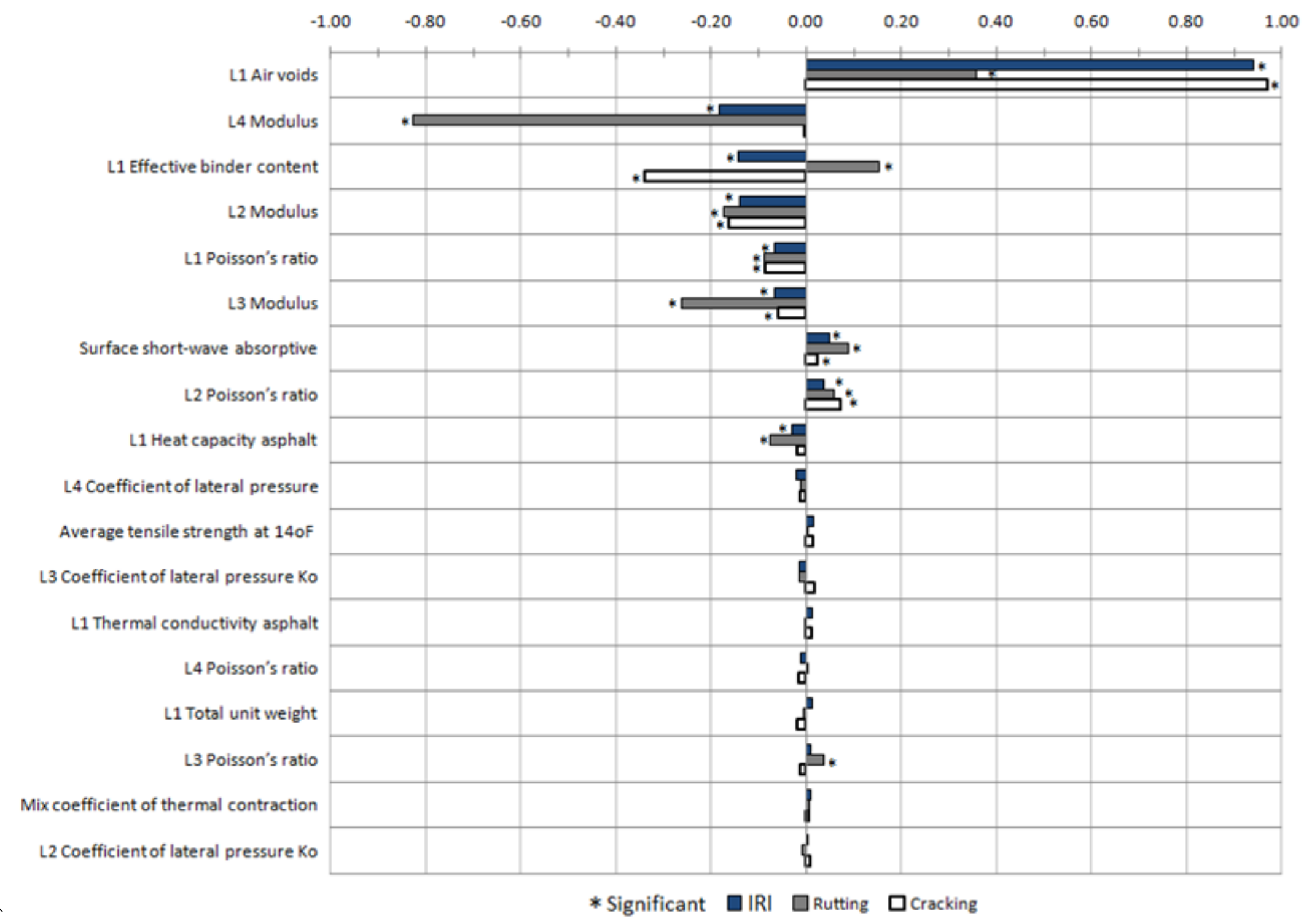

FIGURE 7.10 Standardized Regression Coefficients for Structure 2 
Tables 7.11 and 7.12 present the material properties for each layer in structure 1 and 2 respectively and their sensitivity to rutting, cracking, and IRI according to the sign of the SRC. A positive sign (+) indicates that the parameter is significant with a positive SRC. A negative sign (-) indicates that the parameter is significant with a negative SRC. The letter $\mathrm{n}$ indicates that the parameter is not significant. A positive SRC indicates that the corresponding MEPDG output increases as the input value for the parameter increases. A negative SRC indicates that the corresponding MEPDG output decreases as the input value for the parameter increases. For example, surface short-wave absorptive in layer $\mathrm{L}_{1}$ has signs,++ , and + in Table 7.11 for rutting, cracking, and IRI, so an increase in the input value of this parameter would lead to increase in rutting, cracking, and IRI. A decrease in this input value would lead to contrary effects on these MEPDG outputs. Thermal conductivity of asphalt in layer $\mathrm{L}_{1}$ has signs,$+ \mathrm{n}$, and + thus an increase in input value of this parameter would lead to increase in rutting and IRI, and has not significant effect on cracking. Parameters with n, n, and $n$ are not significant for any of the MEPDG outputs considered in this study, IRI, rutting, and cracking. Other input parameters have significant effects in all MEPDG outputs in this study. 
TABLE 7.11 Effect of Material Input Parameters in MEPDG Outputs on Structure 1

\begin{tabular}{|c|c|c|c|c|}
\hline \multirow{2}{*}{ LAYER } & \multirow{2}{*}{ PARAMETER } & \multicolumn{3}{|c|}{ Sign of SRC } \\
\hline & & RUTTING & CRACKING & $\overline{\text { IRI }}$ \\
\hline $\mathbf{L 1}$ & Surface short-wave absorptive & + & + & + \\
\hline \multirow{6}{*}{$\mathbf{L 1}$} & L1 Effective binder content & + & + & + \\
\hline & L1 Air Voids & + & + & + \\
\hline & L1 Total unit weight & $\mathrm{n}$ & - & $\mathrm{n}$ \\
\hline & L1 Poisson's ratio & - & - & - \\
\hline & L1 Thermal conductivity as phalt & + & $\mathrm{n}$ & $\mathrm{n}$ \\
\hline & L1 Heat capacity asphalt & - & $\mathrm{n}$ & $\mathrm{n}$ \\
\hline \multirow{6}{*}{$\mathbf{L 2}$} & L2 Effective binder content & + & $\mathrm{n}$ & + \\
\hline & L2 Air voids & + & + & + \\
\hline & L2 Total unit weight & $\mathrm{n}$ & $\mathrm{n}$ & $\mathrm{n}$ \\
\hline & L2 Poisson's ratio & - & - & - \\
\hline & L2 Thermal conductivity asphalt & $\mathrm{n}$ & $\mathrm{n}$ & $\mathrm{n}$ \\
\hline & L2 Heat capacity asphalt & - & $\mathrm{n}$ & $\mathrm{n}$ \\
\hline \multirow{6}{*}{$\mathbf{L 3}$} & L3 Effective binder content & $\mathrm{n}$ & + & + \\
\hline & L3 Air voids & $\mathrm{n}$ & + & + \\
\hline & L3 Total unit weight (pcf) & $\mathrm{n}$ & $\mathrm{n}$ & $\mathrm{n}$ \\
\hline & L3 Poisson's ratio & $\mathrm{n}$ & - & - \\
\hline & L3 Thermal conductivity as phalt & $\mathrm{n}$ & $\mathrm{n}$ & $\mathrm{n}$ \\
\hline & L3 Heat capacity asphalt & $\mathrm{n}$ & $\mathrm{n}$ & $\mathrm{n}$ \\
\hline \multirow{6}{*}{$\mathbf{L} \mathbf{4}$} & L4 Effective binder content & $\mathrm{n}$ & - & - \\
\hline & L4 Air voids & $\mathrm{n}$ & $\mathrm{n}$ & $\mathrm{n}$ \\
\hline & L4 Total unit weight & $\mathrm{n}$ & $\mathrm{n}$ & $\mathrm{n}$ \\
\hline & L4 Pois son's ratio & $\mathrm{n}$ & - & - \\
\hline & L4 Thermal conductivity asphalt & $\mathrm{n}$ & $\mathrm{n}$ & $\mathrm{n}$ \\
\hline & L4 Heat capacity asphalt & $\mathrm{n}$ & $\mathrm{n}$ & $\mathrm{n}$ \\
\hline \multirow{5}{*}{$\mathbf{L 5}$} & L5 Poisson's ratio & $\mathrm{n}$ & $\mathrm{n}$ & $\mathrm{n}$ \\
\hline & L5 Coefficient of lateral pressure Ko & $\mathrm{n}$ & $\mathrm{n}$ & $\mathrm{n}$ \\
\hline & L5 Modulus & - & - & - \\
\hline & Average tensile strength at $14 \mathrm{oF}$ & $\mathrm{n}$ & $\mathrm{n}$ & $\mathrm{n}$ \\
\hline & Mix coefficient of thermal contraction & $\mathrm{n}$ & $\mathrm{n}$ & $\mathrm{n}$ \\
\hline
\end{tabular}


TABLE 7.12 Effect of Material Input Parameters in MEPDG Outputs on Structure 2

\begin{tabular}{|c|c|c|c|c|}
\hline \multirow{2}{*}{ LAYER } & \multirow{2}{*}{ PARAMETER } & \multicolumn{3}{|c|}{ Sign of SRC } \\
\hline & & RUTTING & CRACKING & IRI \\
\hline $\mathbf{L 1}$ & Surface short-wave absorptive & + & $\mathrm{n}$ & + \\
\hline \multirow{6}{*}{$\mathbf{L 1}$} & L1 Effective binder content & + & - & - \\
\hline & L1 Air voids & + & + & + \\
\hline & L1 Total unit weight & $\mathrm{n}$ & $\mathrm{n}$ & $\mathrm{n}$ \\
\hline & L1 Pois son's ratio & - & - & - \\
\hline & L1 Thermal conductivity asphalt & $\mathrm{n}$ & $\mathrm{n}$ & $\mathrm{n}$ \\
\hline & L1 Heat capacity as phalt & - & $\mathrm{n}$ & - \\
\hline \multirow{3}{*}{$\mathrm{L} 2$} & L2 Pois son's ratio & + & + & + \\
\hline & L2 Coefficient of lateral pressure (Ko) & $\mathrm{n}$ & $\mathrm{n}$ & $\mathrm{n}$ \\
\hline & L2 Modulus & - & - & - \\
\hline \multirow{3}{*}{$\mathbf{L 3}$} & L3 Poisson's ratio & + & $\mathrm{n}$ & $\mathrm{n}$ \\
\hline & L3 Coefficient of lateral pressure (Ko) & $\mathrm{n}$ & $\mathrm{n}$ & $\mathrm{n}$ \\
\hline & L3 Modulus & - & - & - \\
\hline \multirow{5}{*}{$\mathbf{L} \mathbf{4}$} & L4 Poisson's ratio & $\mathrm{n}$ & $\mathrm{n}$ & $\mathrm{n}$ \\
\hline & L4 Coefficient of lateral pressure (Ko) & $\mathrm{n}$ & $\mathrm{n}$ & $\mathrm{n}$ \\
\hline & L4 Modulus & - & $\mathrm{n}$ & - \\
\hline & Average tensile strength at $14 \mathrm{oF}$ & $\mathrm{n}$ & $\mathrm{n}$ & $\mathrm{n}$ \\
\hline & Mix coefficient of thermal contraction & $\mathrm{n}$ & $\mathrm{n}$ & $\mathrm{n}$ \\
\hline
\end{tabular}

n: not significant

\subsection{Recommended Hierarchical Levels for Material Parameters}

The results of sensitivity analysis were used to identify recommended hierarchical levels for input parameters in this research. A defined methodology to choose hierarchical levels for input parameters in MEPDG does not exist at this time. The approach in this research was as follows:

1. Highly significant parameters with $\mathrm{P}<0.0001$ were assigned to hierarchical level 1

2. Significant parameters with $P \geq 0.0001$ were assigned to hierarchical level 2

3. Not significant parameters were assigned to hierarchical level 3

This approach is based on the idea that parameters which do not affect significantly an MEPDG output may be given any reasonable input value without considerably affecting the MEPDG output. Input values for highly significant parameters have large effects in the MEPDG predictions therefore these parameters were assigned to level 1. 
Table 7.13 and 7.14 presents the assigned hierarchical levels for the material parameters of structures 1 and 2 respectively. The hierarchical levels were assigned for each parameter and distress. The overall hierarchical levels were defined as the highest level from all distresses.

TABLE 7.13 Recommended Hierarchical Levels for Material Parameters for Structure 1

\begin{tabular}{|c|c|c|c|c|c|}
\hline \multirow{2}{*}{ LAYER } & \multirow{2}{*}{ PARAMETER } & \multicolumn{4}{|c|}{ Structure 1} \\
\hline & & RUTTING & CRACKING & IRI & OVERALL \\
\hline Ll & Surface Surface short-wave absorptive & 1 & 1 & 1 & 1 \\
\hline \multirow{6}{*}{$\mathrm{Ll}$} & L1 Effective binder content & 1 & 2 & 1 & 1 \\
\hline & L1 Air Voids & 1 & 1 & 1 & 1 \\
\hline & L1 Total unit weight & 3 & 2 & 3 & 2 \\
\hline & L1 Poisson's ratio & 1 & 2 & 1 & 1 \\
\hline & L1 Thermal conductivity asphalt & 2 & 3 & 3 & 2 \\
\hline & L1 Heat capacity asphalt & 1 & 3 & 3 & 1 \\
\hline \multirow{6}{*}{ L2 } & L2 Effective binder content & 1 & 3 & 1 & 1 \\
\hline & L2 Air voids & 1 & 2 & 1 & 1 \\
\hline & L2 Total unit weight & 3 & 3 & 3 & 3 \\
\hline & L2 Poisson's ratio & 1 & 2 & 1 & 1 \\
\hline & L2 Thermal conductivity asphalt & 3 & 3 & 3 & 3 \\
\hline & L2 Heat capacity asphalt & 2 & 3 & 3 & 2 \\
\hline \multirow{6}{*}{ L3 } & L3 Effective binder content & 3 & 1 & 1 & 1 \\
\hline & L3 Air voids & 3 & 1 & 1 & 1 \\
\hline & L3 Total unit weight (pcf) & 3 & 3 & 3 & 3 \\
\hline & L3 Poisson's ratio & 3 & 2 & 2 & 2 \\
\hline & L3 Thermal conductivity asphalt & 3 & 3 & 3 & 3 \\
\hline & L3 Heat capacity asphalt & 3 & 3 & 3 & 3 \\
\hline \multirow{6}{*}{ L4 } & L4 Effective binder content & 3 & 1 & 1 & 1 \\
\hline & L4 Air voids & 3 & 3 & 3 & 3 \\
\hline & L4 Total unit weight & 3 & 3 & 3 & 3 \\
\hline & L4 Poisson's ratio & 3 & 1 & 2 & 1 \\
\hline & L4 Thermal conductivity asphalt & 3 & 3 & 3 & 3 \\
\hline & L4 Heat capacity asphalt & 3 & 3 & 3 & 3 \\
\hline \multirow{5}{*}{ L5 } & L5 Poisson's ratio & 3 & 3 & 3 & 3 \\
\hline & L5 Coefficient of lateral pressure Ko & 3 & 3 & 3 & 3 \\
\hline & L5 Modulus & 1 & 1 & 1 & 1 \\
\hline & Average tensile strength at $14 \mathrm{oF}$ & 3 & 3 & 3 & 3 \\
\hline & Mix coefficient of thermal contraction & 3 & 3 & 3 & 3 \\
\hline
\end{tabular}


TABLE 7.14 Recommended Hierarchical Levels for Material Parameters for Structure 2

\begin{tabular}{|c|c|c|c|c|c|}
\hline \multirow{2}{*}{ LAYER } & \multirow{2}{*}{ PARAMETER } & \multicolumn{4}{|c|}{ Sign of SRC } \\
\hline & & RUTTING & CRACKING & IRI & OVERALL \\
\hline Ll & Surface short-wave absorptive & 1 & 3 & 1 & 1 \\
\hline \multirow{6}{*}{ Ll } & L1 Effective binder content & 1 & 1 & 1 & 1 \\
\hline & L1 Air voids & 1 & 1 & 1 & 1 \\
\hline & L1 Total unit weight & 3 & 3 & 3 & 3 \\
\hline & L1 Poisson's ratio & 1 & 1 & 1 & 1 \\
\hline & L1 Thermal conductivity asphalt & 3 & 3 & 3 & 3 \\
\hline & L1 Heat capacity asphalt & 1 & 3 & 1 & 1 \\
\hline \multirow{3}{*}{ L2 } & L2 Poisson's ratio & 1 & 1 & 1 & 1 \\
\hline & L2 Coefficient of lateral pressure (Ko) & 3 & 3 & 3 & 3 \\
\hline & L2 Modulus & 1 & 1 & 1 & 1 \\
\hline \multirow{3}{*}{ L3 } & L3 Poisson's ratio & 1 & 3 & 3 & 1 \\
\hline & L3 Coefficient of lateral pressure (Ko) & 3 & 3 & 3 & 3 \\
\hline & L3 Modulus & 1 & 1 & 1 & 1 \\
\hline \multirow{5}{*}{ L4 } & L4 Poisson's ratio & 3 & 3 & 3 & 3 \\
\hline & L4 Coefficient of lateral pressure (Ko) & 3 & 3 & 3 & 3 \\
\hline & L4 Modulus & 1 & 3 & 1 & 1 \\
\hline & Average tensile strength at $14 \mathrm{oF}$ & 3 & 3 & 3 & 3 \\
\hline & Mix coefficient of thermal contraction & 3 & 3 & 3 & 3 \\
\hline
\end{tabular}




\section{CHAPTER 8: MATERIAL PROPERTY DESIRABILITY}

This chapter presents the concept of material properties desirability. First, the results from the sensitivity analysis are used to identify the desirable material properties for a given pavement structure in order to have good performance. Then, Inputs 11 and 181 from the sensitivity analysis of structure 1, which had high predicted distresses compared with all other runs, are analyzed and rerun in MEPDG with the desirable material inputs values. Finally, the results from this analysis are discussed.

\subsection{The Concept of Material Property Desirability}

The important effects of input parameters were studied in Chapter 7. Some parameters are significant while others parameters are not. The signs of the SRC were also identified. It was shown that a positive SRC indicates that the corresponding MEPDG output increases as the input value for the parameter increases, and vice versa. A negative SRC indicates that the corresponding MEPDG output decreases as the input value for the parameter increases, and vice versa. The information from Chapter 7 can be used to identify the most suitable combination of input parameters in order to design a structure which performs well.

Pavement material desirability analysis intends to identify the best combination of material properties in order to design a structure that is predicted to perform well in field. Although it is possible to identified desirable material properties using this methodology, it may be not easy to find materials to meet these desirable material properties. Designers may try to find the combination of material properties for construction as close to the desirable properties as possible.

\subsubsection{Material Properties Desirability}

Structure 1 is used to explain the procedure to identify the desirable material properties for a given pavement structure. Table 8.1 shows the results from the sensitivity analysis. The signs of the SRC are displayed in the table along with the lowest and highest values from the ranges used in the sensitivity analysis. As explained in Chapter 7, a positive sign (+) indicates that the parameter is significant with a positive SRC. 
TABLE 8.1 Material Properties Desirability for Structure 1

\begin{tabular}{|c|c|c|c|c|c|c|c|c|c|c|c|c|}
\hline \multirow{2}{*}{ LAYER } & \multirow{2}{*}{ PARAMETER } & \multirow{2}{*}{ UNITS } & \multicolumn{2}{|c|}{ Range } & \multicolumn{3}{|c|}{ Sign of SRC } & \multicolumn{3}{|c|}{ Desirability } & \multirow{2}{*}{$\begin{array}{l}\text { Range } \\
\text { Side }\end{array}$} & \multirow{2}{*}{$\begin{array}{c}\text { Desirable } \\
\text { Input }\end{array}$} \\
\hline & & & Low & High & RUTTING & CRACKING & IRI & RUTTING & CRACKING & IRI & & \\
\hline $\mathbf{L 1}$ & Surface short-wave absorptive & - & 0.8 & 0.9 & + & + & + & 0.8 & 0.8 & 0.8 & Low & 0.8 \\
\hline \multirow{6}{*}{$\mathbf{L 1}$} & L1 Effective binder content & $\%$ & 4.5 & 6.5 & + & + & + & 4.5 & 4.5 & 4.5 & Low & 4.5 \\
\hline & L1 Air Voids & $\%$ & 3 & 10 & + & + & + & 3 & 3 & 3 & Low & 3 \\
\hline & L1 Total unit weight & pcf & 145 & 150 & $\mathrm{n}$ & - & $\mathrm{n}$ & $\mathrm{n}$ & 150 & $\mathrm{n}$ & High & 150 \\
\hline & L1 Poisson's ratio & - & 0.25 & 0.4 & - & - & - & 0.4 & 0.4 & 0.4 & High & 0.4 \\
\hline & L1 Thermal conductivity asphalt & BTU/hr-ft-F & 0.5 & 0.8 & + & $\mathrm{n}$ & $\mathrm{n}$ & 0.5 & $\mathrm{n}$ & $\mathrm{n}$ & Low & 0.5 \\
\hline & L1 Heat capacity asphalt & BT U/lb-F ${ }^{0}$ & 0.22 & 0.5 & - & $\mathrm{n}$ & $\mathrm{n}$ & 0.5 & $\mathrm{n}$ & $\mathrm{n}$ & High & 0.5 \\
\hline \multirow{6}{*}{$\mathbf{L 2}$} & L2 Effective binder content & $\%$ & 3.5 & 6 & + & $\mathrm{n}$ & + & 3.5 & $\mathrm{n}$ & 3.5 & Low & 3.5 \\
\hline & L2 Air voids & $\%$ & 3 & 10 & + & + & + & 3 & 3 & 3 & Low & 3 \\
\hline & L2 Total unit weight & pcf & 145 & 150 & $\mathrm{n}$ & $\mathrm{n}$ & $\mathrm{n}$ & $\mathrm{n}$ & $\mathrm{n}$ & $\mathrm{n}$ & $\mathrm{n}$ & $\mathrm{n}$ \\
\hline & L2 Poisson's ratio & - & 0.25 & 0.4 & - & - & - & 0.4 & 0.4 & 0.4 & High & 0.4 \\
\hline & L2 Thermal conductivity asphalt & BT U/hr-ft-F & 0.5 & 0.8 & $\mathrm{n}$ & $\mathrm{n}$ & $\mathrm{n}$ & $\mathrm{n}$ & $\mathrm{n}$ & $\mathrm{n}$ & $\mathrm{n}$ & $\mathrm{n}$ \\
\hline & L2 Heat capacity asphalt & BT U/lb-F & 0.22 & 0.4 & - & $\mathrm{n}$ & $\mathrm{n}$ & 0.4 & $\mathrm{n}$ & $\mathrm{n}$ & High & 0.4 \\
\hline \multirow{6}{*}{$\mathbf{L 3}$} & L3 Effective binder content & $\%$ & 2.5 & 4 & $\mathrm{n}$ & + & + & $\mathrm{n}$ & 2.5 & 2.5 & Low & 2.5 \\
\hline & L3 Air voids & $\%$ & 6 & 12 & $\mathrm{n}$ & + & $\mathrm{n}$ & $\mathrm{n}$ & 6 & $\mathrm{n}$ & Low & 6 \\
\hline & L3 Total unit weight (pcf) & $\mathrm{pcf}$ & 145 & 150 & $\mathrm{n}$ & $\mathrm{n}$ & $\mathrm{n}$ & $\mathrm{n}$ & $\mathrm{n}$ & $\mathrm{n}$ & $\mathrm{n}$ & $\mathrm{n}$ \\
\hline & L3 Poisson's ratio & - & 0.25 & 0.4 & $\mathrm{n}$ & - & - & $\mathrm{n}$ & 0.4 & 0.4 & High & 0.4 \\
\hline & L3 Thermal conductivity as phalt & BTU/hr-ft-F & 0.5 & 0.8 & $\mathrm{n}$ & $\mathrm{n}$ & $\mathrm{n}$ & $\mathrm{n}$ & $\mathrm{n}$ & $\mathrm{n}$ & $\mathrm{n}$ & $\mathrm{n}$ \\
\hline & L3 Heat capacity asphalt & BT U/lb-F & 0.22 & 0.5 & $\mathrm{n}$ & $\mathrm{n}$ & $\mathrm{n}$ & $\mathrm{n}$ & $\mathrm{n}$ & $\mathrm{n}$ & $\mathrm{n}$ & $\mathrm{n}$ \\
\hline \multirow{6}{*}{$\mathbf{L} \mathbf{4}$} & L4 Effective binder content & $\%$ & 2 & 3 & $\mathrm{n}$ & - & - & $\mathrm{n}$ & 3 & 3 & High & 3 \\
\hline & L4 Air voids & $\%$ & 15 & 20 & $\mathrm{n}$ & $\mathrm{n}$ & $\mathrm{n}$ & $\mathrm{n}$ & $\mathrm{n}$ & $\mathrm{n}$ & $\mathrm{n}$ & $\mathrm{n}$ \\
\hline & L4 Total unit weight & pcf & 145 & 150 & $\mathrm{n}$ & $\mathrm{n}$ & $\mathrm{n}$ & $\mathrm{n}$ & $\mathrm{n}$ & $\mathrm{n}$ & $\mathrm{n}$ & $\mathrm{n}$ \\
\hline & L4 Poisson's ratio & - & 0.25 & 0.4 & $\mathrm{n}$ & - & - & $\mathrm{n}$ & 0.4 & 0.4 & High & 0.4 \\
\hline & L4 Thermal conductivity as phalt & BTU/hr-ft-F & 0.5 & 0.8 & $\mathrm{n}$ & $\mathrm{n}$ & $\mathrm{n}$ & $\mathrm{n}$ & $\mathrm{n}$ & $\mathrm{n}$ & $\mathrm{n}$ & $\mathrm{n}$ \\
\hline & L4 Heat capacity asphalt & BT U/lb-F & 0.22 & 0.4 & $\mathrm{n}$ & $\mathrm{n}$ & $\mathrm{n}$ & $\mathrm{n}$ & $\mathrm{n}$ & $\mathrm{n}$ & $\mathrm{n}$ & $\mathrm{n}$ \\
\hline \multirow{5}{*}{$\mathbf{L 5}$} & L5 Poisson's ratio & - & 0.3 & 0.4 & $\mathrm{n}$ & $\mathrm{n}$ & $\mathrm{n}$ & $\mathrm{n}$ & $\mathrm{n}$ & $\mathrm{n}$ & $\mathrm{n}$ & $\mathrm{n}$ \\
\hline & L5 Coefficient of lateral pressure Ko & - & 0.5 & 0.7 & $\mathrm{n}$ & $\mathrm{n}$ & $\mathrm{n}$ & $\mathrm{n}$ & $\mathrm{n}$ & $\mathrm{n}$ & $\mathrm{n}$ & $\mathrm{n}$ \\
\hline & L5 Modulus & psi & 5000 & 9000 & - & - & - & 9000 & 9000 & 9000 & High & 9000 \\
\hline & Average tensile strength at $14 \mathrm{oF}$ & $\mathrm{psi}$ & 500 & 1500 & $\mathrm{n}$ & $\mathrm{n}$ & $\mathrm{n}$ & $\mathrm{n}$ & $\mathrm{n}$ & $\mathrm{n}$ & $\mathrm{n}$ & $\mathrm{n}$ \\
\hline & Mix coefficient of thermal contraction & in $/$ in $/{ }^{\circ} F$ & $2.2 \mathrm{E}-05$ & $3.4 \mathrm{E}-05$ & $\mathrm{n}$ & $\mathrm{n}$ & $\mathrm{n}$ & $\mathrm{n}$ & $\mathrm{n}$ & $\mathrm{n}$ & $\mathrm{n}$ & $\mathrm{n}$ \\
\hline
\end{tabular}


A negative sign (-) indicates that the parameter is significant with a negative SRC. The letter $\mathrm{n}$ indicates that the parameter is no significant. A positive SRC indicates that the corresponding MEPDG output increases as the input value increases. A negative SRC indicates that the corresponding MEPDG output decreases as the input value increases.

The signs of the SRC are used to define desirable input values for each MEPDG output (rutting, cracking, and IRI). A significant input parameter with positive SRC would require an input value close to the lowest side of its input range in order have the better effect on performance. A significant input parameter with negative SRC would require an input value close to the highest side of its input range in order have the better effect on performance. Columns under desirability have been defined with this concept in Table 8.1. The lowest or highest values of each parameter input range has been defined as desirable for each MEPDG output. Where the parameter is not significant, $\mathrm{n}$ have been placed in the corresponding cell in the table. If the signs of the SRC are equal for all performance measures, then the desirable input level is a low value for positive SRC or a high value for a negative SRC ignoring all no significant parameters. Inspection of the desirability column in Table 8.1 shows the signs of SRC are equal for each parameter for all distresses. In case of different signs the desirable value should be selected to favor performance for a distress.

The column Range Side is the general result from each input parameter after the analysis of all signs for all MEPDG output. Notice that, all sign are equal within parameter for structure 1. The better effect should be chosen in case of different signs for a given structure. Cells with $n$ were not considered in the definition of the range side. The column Desirable Input was defined according to the range side. It is clear that the expected desirable input values need to be close to these values but not necessarily the same value.

Runs 11 and 181 from the sensitivity analysis of structure 1 were evaluated using the concept of material property desirability. The column Runs in Table 8.2 shows the input values for runs 11 an 181 used in the sensitivity analysis. A boundary of one third of the input range was used in order to check how far the original values are from the desirable values. Lowest value plus one third of the input range was defined as acceptable for the low rage side. Highest value minus one third of the input range was defined as acceptable for the high range side. 
TABLE 8.2 Checking Runs 11 and 181 of Structure 1

\begin{tabular}{|c|c|c|c|c|c|c|c|c|c|c|c|c|}
\hline \multirow{2}{*}{ LAYER } & \multirow{2}{*}{ PARAMETER } & \multirow{2}{*}{ UNITS } & \multicolumn{2}{|c|}{ Range } & \multirow{2}{*}{$\begin{array}{c}\text { Range } \\
\text { Side }\end{array}$} & \multirow{2}{*}{\begin{tabular}{|c|} 
Desirable \\
Input
\end{tabular}} & \multicolumn{2}{|c|}{ RUNS } & \multicolumn{2}{|c|}{ Run Checking } & \multicolumn{2}{|c|}{ New Runs } \\
\hline & & & Low & High & & & 11 & 181 & 11 & 181 & 11 & 181 \\
\hline L1 & Surface short-wave absorptive & - & 0.8 & 0.9 & Low & 0.8 & 0.88 & 0.84 & $\mathrm{X}$ & $\mathrm{X}$ & 0.80 & 0.8 \\
\hline \multirow{6}{*}{ L1 } & L1 Effective binder content & $\%$ & 4.5 & 6.5 & Low & 4.5 & 6.4 & 4.7 & $\mathrm{X}$ & $\mathrm{X}$ & 4.5 & 4.5 \\
\hline & L1 Air Voids & $\%$ & 3 & 10 & Low & 3 & 9.9 & 8.2 & $\mathrm{X}$ & $\mathrm{X}$ & 3.0 & 3 \\
\hline & L1 Total unit weight & pcf & 145 & 150 & High & 150 & 148.2 & 147.2 & $\mathrm{OK}$ & $\mathrm{X}$ & 148.2 & 150 \\
\hline & L1 Poisson's ratio & \begin{tabular}{|l|}
- \\
\end{tabular} & 0.25 & 0.4 & High & 0.4 & 0.35 & 0.34 & $\mathrm{OK}$ & $\mathrm{X}$ & 0.35 & 0.4 \\
\hline & L1 Thermal conductivity asphalt & BTU/hr-ft-F & 0.5 & 0.8 & Low & 0.5 & 0.66 & 0.70 & $\mathrm{X}$ & $\mathrm{X}$ & 0.50 & 0.5 \\
\hline & L1 Heat capacity asphalt & BTU/lb-F ${ }^{\mathrm{o}}$ & 0.22 & 0.5 & High & 0.5 & 0.27 & 0.33 & $\mathrm{X}$ & $\mathrm{X}$ & 0.50 & 0.5 \\
\hline \multirow{6}{*}{$\mathbf{L 2}$} & L2 Effective binder content & $\%$ & 3.5 & 6 & Low & 3.5 & 5.8 & 5.2 & $\mathrm{X}$ & $\mathrm{X}$ & 3.5 & 3.5 \\
\hline & L2 Air voids & $\%$ & 3 & 10 & Low & 3 & 7.3 & 9.1 & $\mathrm{X}$ & $\mathrm{X}$ & 3.0 & 3 \\
\hline & L2 Total unit weight & pcf & 145 & 150 & $\mathrm{n}$ & $\mathrm{n}$ & 147.3 & 146.3 & $\mathrm{n}$ & $\mathrm{n}$ & 147.3 & 146.3 \\
\hline & L2 Poisson's ratio & - & 0.25 & 0.4 & High & 0.4 & 0.26 & 0.35 & $\mathrm{X}$ & $\mathrm{OK}$ & 0.40 & 0.35 \\
\hline & L2 Thermal conductivity as phalt & BTU/hr-ft-F & 0.5 & 0.8 & $\mathrm{n}$ & $\mathrm{n}$ & 0.70 & 0.68 & $\mathrm{n}$ & $\mathrm{n}$ & 0.70 & 0.68 \\
\hline & L2 Heat capacity asphalt & BTU/lb-F ${ }^{\mathrm{o}}$ & 0.22 & 0.4 & High & 0.4 & 0.36 & 0.31 & OK & $\mathrm{X}$ & 0.36 & 0.4 \\
\hline \multirow{6}{*}{$\mathbf{L 3}$} & L3 Effective binder content & $\%$ & 2.5 & 4 & Low & 2.5 & 3.4 & 4.0 & $\mathrm{X}$ & $\mathrm{X}$ & 2.5 & 2.5 \\
\hline & L3 Air voids & $\%$ & 6 & 12 & Low & 6 & 10.5 & 11.7 & $\mathrm{X}$ & $\mathrm{X}$ & 6.0 & 6 \\
\hline & L3 Total unit weight (pcf) & pcf & 145 & 150 & $\mathrm{n}$ & $\mathrm{n}$ & 149.8 & 147.5 & $\mathrm{n}$ & $\mathrm{n}$ & 149.8 & 147.5 \\
\hline & L3 Poisson's ratio & - & 0.25 & 0.4 & High & 0.4 & 0.39 & 0.32 & $\mathrm{OK}$ & $\mathrm{X}$ & 0.39 & 0.4 \\
\hline & L3 Thermal conductivity as phalt & BTU/hr-ft-F & 0.5 & 0.8 & $\mathrm{n}$ & $\mathrm{n}$ & 0.74 & 0.69 & $\mathrm{n}$ & $\mathrm{n}$ & 0.74 & 0.69 \\
\hline & L3 Heat capacity as phalt & BTU/lb-F ${ }^{\circ}$ & 0.22 & 0.5 & $\mathrm{n}$ & $\mathrm{n}$ & 0.29 & 0.35 & $\mathrm{n}$ & $\mathrm{n}$ & 0.29 & 0.35 \\
\hline \multirow{6}{*}{$\mathbf{L A}$} & L4 Effective binder content & $\%$ & 2 & 3 & High & 3 & 2.2 & 2.1 & $\mathrm{X}$ & $\mathrm{X}$ & 3.0 & 3 \\
\hline & L4 Air voids & $\%$ & 15 & 20 & $\mathrm{n}$ & $\mathrm{n}$ & 16.8 & 19.5 & $\mathrm{n}$ & $\mathrm{n}$ & 16.8 & 19.5 \\
\hline & L4 Total unit weight & pcf & 145 & 150 & $\mathrm{n}$ & $\mathrm{n}$ & 147.3 & 149.6 & $\mathrm{n}$ & $\mathrm{n}$ & 147.3 & 149.6 \\
\hline & L4 Poisson's ratio & - & 0.25 & 0.4 & High & 0.4 & 0.29 & 0.39 & $\mathrm{X}$ & $\mathrm{OK}$ & 0.40 & 0.39 \\
\hline & L4 Thermal conductivity as phalt & BTU/hr-ft-F & 0.5 & 0.8 & $\mathrm{n}$ & $\mathrm{n}$ & 0.68 & 0.53 & $\mathrm{n}$ & $\mathrm{n}$ & 0.68 & 0.53 \\
\hline & L4 Heat capacity asphalt & BTU/lb-F ${ }^{\circ}$ & 0.22 & 0.4 & $\mathrm{n}$ & $\mathrm{n}$ & 0.31 & 0.33 & $\mathrm{n}$ & $\mathrm{n}$ & 0.31 & 0.33 \\
\hline \multirow{5}{*}{$\mathbf{L 5}$} & L5 Poisson's ratio & - & 0.3 & 0.4 & $\bar{n}$ & $\mathrm{n}$ & 0.37 & 0.34 & $\bar{n}$ & $\bar{n}$ & 0.37 & 0.34 \\
\hline & L5 Coefficient of lateral pressure Ko & - & 0.5 & 0.7 & $\mathrm{n}$ & $\mathrm{n}$ & 0.50 & 0.50 & $\mathrm{n}$ & $\mathrm{n}$ & 0.50 & 0.5 \\
\hline & L5 Modulus & psi & 5000 & 9000 & High & 9000 & 5202 & 5322 & $\mathrm{X}$ & $\mathrm{X}$ & 9000 & 9000 \\
\hline & Average tensile strength at $14 \mathrm{oF}$ & psi & 500 & 1500 & $\mathrm{n}$ & $\mathrm{n}$ & 1388.9 & 731.2 & $\mathrm{n}$ & $\mathrm{n}$ & 1388.9 & 731.2 \\
\hline & Mix coefficient of thermal contraction & $\mathrm{in} / \mathrm{in} /{ }^{\circ} \mathrm{F}$ & 2.2E-05 & 3.4E-05 & $\mathrm{n}$ & $\mathrm{n}$ & $2.8 \mathrm{E}-05$ & $3.0 \mathrm{E}-05$ & $\mathrm{n}$ & $\mathrm{n}$ & $2.8 \mathrm{E}-05$ & 3.0E-05 \\
\hline
\end{tabular}


Cells that meet these criteria were defined as "OK", cells that fail to meet these criteria were identified with a " $\mathrm{X}$ ", and cells in which the input parameter is not significant were identified with " $n$ ". Both runs 11 and 181 fail in most of the input parameters (column Run Checking).

New runs were defined in order to check runs 11 and 181 with the desirable input values in MEPDG. Input values that fail to meet the desirable criteria were replaced with their corresponding desirable value. Input values that meet desirable criteria or are not significant were not altered. The column New Runs in Table 8.2 shows the new input values. The new runs were input to MEPDG.

Figure 8.1 displays the distribution of MEPDG outputs in the sensitivity analysis for all 300 runs. Outputs of runs 11 and 181 are extreme values with the highest IRI and cracking. The output of rutting does not show extreme values for these two runs. Figure 8.2 displays the distribution of MEPDG output of IRI, rutting, and cracking for all 300 runs but the outputs for runs 11 and 181 were replaced for the outputs with the desirable input values. Figure 8.2 shows very good predicted performance for MEPDG with the desirable input values. The new predicted performance is very low for IRI and rutting and there are not extreme values for cracking.

This verification provides additional proof that the sensitivity of the material properties are well identified with the methodology developed in this research. Additionally, this verification allows a better knowledge for the choice of materials in a given pavement structure. 

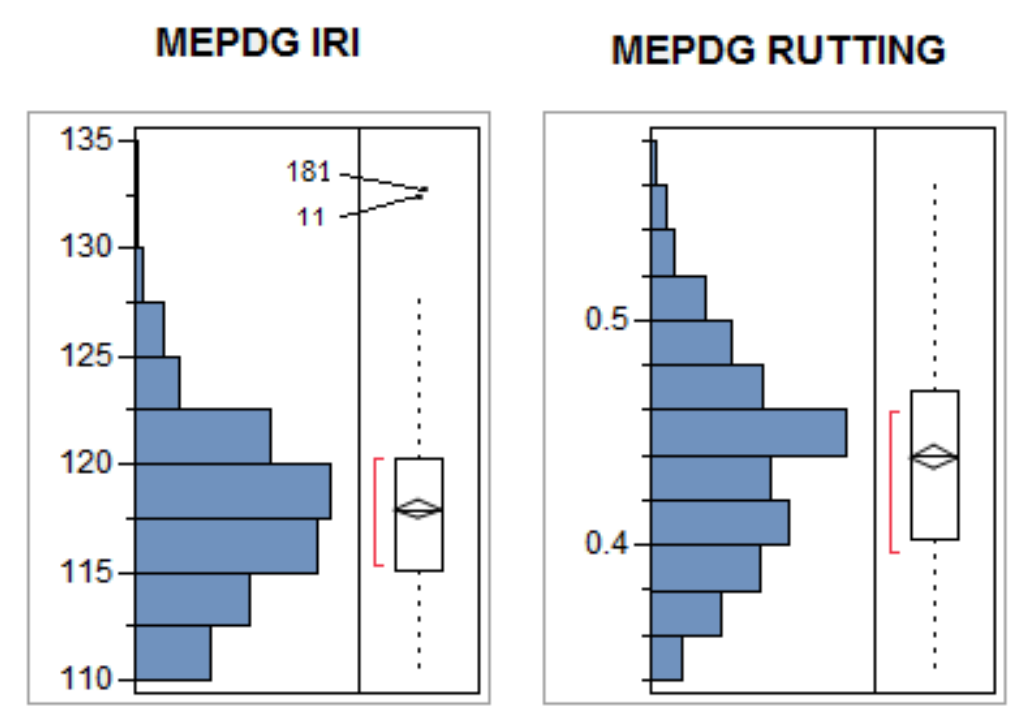

\section{MEPDG CRACKING}

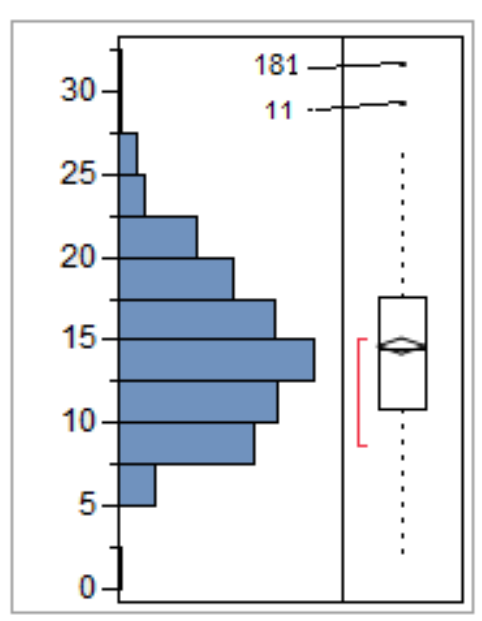

FIGURE 8.1 Distribution of MEPDG Outputs

MEPDG IRI

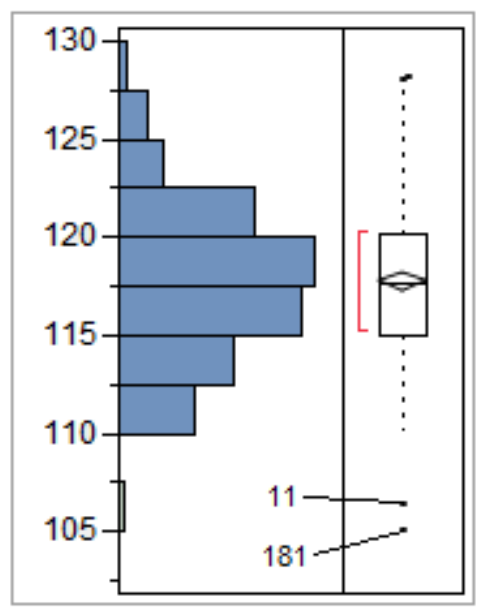

MEPDG RUTTING

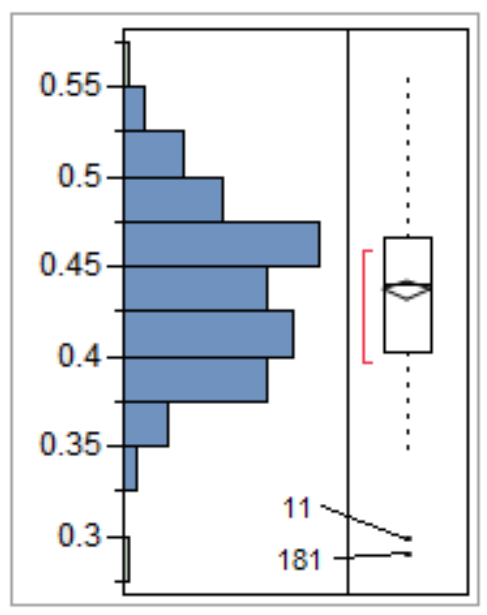

MEPDG CRACKING

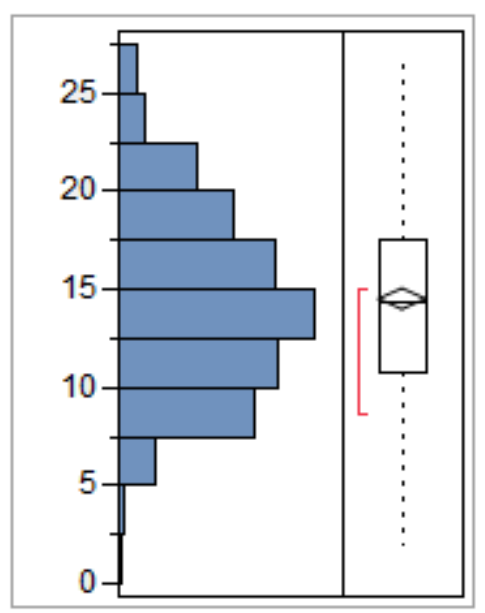

FIGURE 8.2 Distribution of MEPDG Outputs with the New Runs 11 and 181 


\section{CHAPTER 9: CONCLUSIONS}

The Mechanistic-Empirical Pavement Design Guide (MEPDG) is a complex pavement design procedure that requires many input parameters. The number of parameters varies depending on the type of pavement structure. There is a large amount of uncertainty in the determination of inputs and the prediction of pavement performance. The pavement distress models require local calibration to most accurately predict pavement distresses. There is need for identification of the most sensitive parameters in order to address calibration, facilitate data collection, characterize input parameters, determine recommended hierarchical level for input parameters, and for a general understanding of MEPDG.

Several researchers have addressed sensitivity analyses of MEPDG. Most of them have used the technique of varying the parameters one at time while keeping the other parameters unchanged. This technique is limited and requires many runs to analyze several input parameters. Another approach followed in previous research has been factorial experiments based on analysis of variance (ANOVA). The problem with factorial experiment is that MEPDG has a large number of parameters and parameter combinations making intractable a complete analysis of MEPDG with factorial experiments because of the huge size of experiments required. Additionally, the ANOVA technique for factorial experiments is based on the analysis of the variability due to experimental error. Since MEPDG is computer code, any time it is run with the same set of input parameters, the same output is obtained. So, there is no experimental error and this type of ANOVA may not be suitable for MEPDG sensitivity.

A better approach to address sensitivity of complex computer codes, such as MEPDG, is to use space-filling experiments based on random sampling of the entire input space, along with metamodeling and sensitivity analysis techniques. One of the most common techniques for random sampling for computer runs is Monte Carlo Simulations and its improvements such as Latin Hypercube Sampling. The difference between Monte Carlo and Latin Hypercube sampling is that Monte Carlo is simple random sampling while Latin Hypercube is a stratified random sampling which does a better job in space filling experiments to sample the entire space of the parameter with a smaller sample size. 
Multiple Regression Analysis with Standardized Regression Coefficients (SRC) is a practical technique for metamodeling and sensitivity analysis of MEPDG. A good regression model is required in order to have reliable results. Rank transformation may be used in case of a poor regression fit. Generally, rank transformations had better multiple regression coefficients $\left(\mathrm{R}^{2}\right)$ than the original data in this study.

The magnitude of the SRC reveals the relative importance among input parameters; higher SRC have larger effects. The sign of the SRC shows the effect of increasing or decreasing a given input value on the predicted distress from MEPDG. A positive SRC indicates that as the input value increases the predicted MEPDG output increases and vice versa.

In general, traffic input parameters correlate well with MEPDG predicted rutting. Nonlinearities were found for cracking in structure 1 and for IRI and cracking in structure 2. Rank transformations removed nonlinearities and improved the multiple correlation coefficients, $\mathrm{R}^{2}$. The good regression models explain much of the variation of the model outputs in response to their inputs. The results are consistent for all distresses and structures in the study. Two-way AADT, traffic growth, percent of trucks in the design direction, percent of heavy vehicles, and percent of trucks in the design lane are significant effects with positive SRC for all distresses on both structures. Dual tire spacing and traffic wander are significant effects with negative SRC for all distresses and both structures. Operational speed was significant for all distresses on structure 1but does not significantly affect any distress on structures 2. Operational speed has negative SRC for all distresses and both structures. Average axle width and mean wheel location do not significantly affect the MEPDG output for all distresses and both structure. The similarities in the results for both structures show the effects of traffic input parameters are independent of the structure configuration.

The sensitivity of MEPDG outputs to material input parameters showed that IRI, rutting, and cracking correlate well with the input parameters for structure 1 . In the case of structure 2, nonlinearities were found for IRI and cracking but the rank transformed data had good correlation for these distresses. Fitting a multiple regression model to a large number of input parameters may result in a poor multiple regression coefficient $\left(\mathrm{R}^{2}\right)$ but rank transformation may overcome this limitation. 
The sensitivity study for structure 1 shows that 13 of the 30 material input parameters have a significant effect on predicted IRI from MEPDG; 6 of the 13 significant parameters have negative SRC while the other 7 significant parameters have positive SRC. The largest effects on IRI in structure 1 were resilient modulus of subgrade (L5), and as-built air voids of L3. In the case of rutting, 11 of the 30 material input parameters have a significant effect on predicted rutting from MEPDG; 5 of the 11 significant parameters have negative SRC while the other 6 significant parameters have positive SRC. The largest effects for rutting in structure 1 were resilient modulus of subgrade (L5), Poisson's ratio of L1, and as-built air voids of L1 and L2. Regarding to cracking, 13 of the 30 material input parameters in the study have a significant effect on predicted cracking from MEPDG in structure 1; 7 of the significant parameters have negative SRC while the other 6 parameters have positive SRC. The material properties with the largest effect on cracking in structure 1 are air voids of layer L3, effective binder content of layers L4, and resilient modulus of subgrade (L5).

In the case of structure 2, out of 18 input parameters 9 are significant for IRI, 10 are significant for rutting, and 7 are significant for cracking. The largest effects on IRI and cracking in structure 2 are due to air voids and effective binder content of layers L1. The largest effects in rutting in structure 2 are due to resilient modulus of subgrade (L4) and as-built air voids of L1.

The study shows that what makes a material property significant is not only the property itself, but also the depth of the layer in the pavement structure. A parameter may be significant for a given distress in a layer and not significant for the same distress in another layer. The material requirements should consider the depth of the material in the pavement structure.

The sign of a very small value of SRC (close to zero) is not accurately predicted by the multiple regression models but this is negligible because parameters with a very small SRC do not significantly affect the MEPDG outputs.

In the case of material sensitivity, the analysis of significance of parameters is made with the MEPDG output using t-test. The magnitudes of those outputs play an important role in this analysis. This clearly identifies interaction between the type of structure and material input parameters. This was not the case in the traffic analysis where the results from the analysis are independent of the structure. 
The findings from this study were used to develop the concept of material property desirability as a tool to help pavement engineers in the choice of materials to design pavement structures that are expected to performance well in field. 


\section{REFERENCES}

1. NCHRP. Guide for Mechanistic-Empirical Design of new and rehabilitated pavement structures. Final Report, NCHRP Project 1-37A. Washington, DC: TRB, National Research Council, 2004.

2. AASHTO. Mechanistic-Empirical pavement design guide: a manual of practice. Washington : AASHTO, 2008.

3. Yoder, Eldon J. and Witczack, Matthew W. Principles of Pavement Design. New York : Wiley \& Sons, Inc, 1975.

4. Huang, Yang H. Pavement Analysis and Design. New Jersey : Pearson Education, 2004.

5. NCHRP. Guide for Mechanistic-Empirical Design of new and rehabilitated pavement structures. Final Report, NCHRP Project 1-37A; Appendix RR: Finite element procedure for flexible pavement analysis. Washington, DC : TRB, National Research Council, 2004.

6. NCHRP. Guide for Mechanistic-Empirical Design of new and rehabilitated pavement structures. Final Report, NCHRP Project 1-37A; Appendix HH; Field calibration of the thermal cracking model. Washington, DC : TRB, National Research Council, 2003.

7. Graves, R. Clark and Mahboub, Kamyar C. Pilot study in Sampling-Based sensitivity analysis of NCHRP design guide for flexible pavements. Washington DC: Transportation Research Record: Jurnal of Transportation Reseaech Board, 2006.

8. Hall, Kevin D and Beam, Steven. Estimating the sensitivity of design input variables for rigid pavement analysis with a mechanistic- empirical design guide. Washington DC: Transportation Reseach Record: Jurnal of the Transportation Research Board, 2005.

9. Papagiannakis, A. T., et al. Sensitivity of NCHRP 1-37A pavement design to traffic input. Washington DC : Transportation Research Record: Jurnal of the Transportation Research Board, 2006.

10. Haider, W. syed, Neeraj, Buch and Karim, Chatii. Simplified approach for quantifying effect of significant input variables and designing rigid pavements using M-E PDG. 88th annual meeting. Washington DC : Transportation Researsh Board, 2009.

11. Montgomery, Douglas C. Design and Analysis of Experiments. Hoboken : Wiley \& Sons, 2005. 
12. Fang, Kai-tai, Li, Runze and Sudjianto, Agus. Desing and Modelling for Computer Experiments. s.1. : Chapman \& Hall, 2006.

13. Sacks, Jerome, et al. Design and Analysis of Computer Experiments. s.1. : Statistical Science, 1989. Vol. 4.

14. Santner, Thomas J, Williams, Brian J and Notz, William I. The Design and Analysis of Computer Experiments. New York : Springer, 2003.

15. Matala, Anna. Sample size requeriment for Monte Carlo - simulation using Latin Hypercube Sampling. Final Report : Helsinki University of Technology, 2008.

16. McWillians, Thomas P. Sensitivity Analysis of Geologic computers models: Aformal procedure based on Latin Hypercube Sampling. Springer Netherlands : Mathematical Geology, 1987. Vol. 19.

17. Iman, R L and Conover, W J. The Use of the Rank Transform in Regression. Washington : Technometrics, 1979. pp. 499-509.

18. Helton, J. C and Iman, R L. Sensitivity Analysis of a Model for the Environmental Movement of Radionuclides. PubMed : Health physics, 1982. pp. 565-584. Vol. 42.

19. Mrawira, Donath, et al. Sensitivity Analysis of Computer Models: World Bank HDM-III Model. Journal of Transportation Engineering. 1999.

20. Welch, William J., et al. Screening, Predicting, and Computer Experiments. Technometrics. 1992, Vol. 34, No.1.

21. Mrawira, Donath. Streamlining the World Bank's high way design and maintenance standards model (HDM-III) for network level application. Waterloo, Canada: Ph.D thesis, University of Waterloo.

22. Draper, Norman R. and Smith, Harry. Applied regression analysis. NY : Wiley \& Sons, 2005. 
Appendix A

\section{PARAMETERS AND RANGES}


TRAFFIC SENSITIVITY - STRUCTURE 1

MATERIAL PROPERTIES AND RANGES

\section{A. Project description}

1 General information

Design life (year)

Base/subgrade construction month

Pavement construction month

Traffic open

Type design: flexible pavement

\begin{tabular}{|c|c|c|}
\hline FIX & \multicolumn{1}{|c|}{ VARY } & Values/Ranges \\
\hline $\mathrm{x}$ & & 20 \\
\hline $\mathrm{x}$ & & May \\
\hline $\mathrm{x}$ & & June \\
\hline $\mathrm{x}$ & & July \\
\hline $\mathrm{x}$ & & Flexible \\
\hline
\end{tabular}

\section{Site project identification}

\begin{tabular}{|l|c|c|c|}
\hline Location & $\mathrm{x}$ & & Morgantown \\
\hline Station/milepost format: miles & $\mathrm{x}$ & & 0.00 \\
\hline Station/milepost begin: & $\mathrm{x}$ & & 0.20 \\
\hline Station/milepost end: & $\mathrm{x}$ & & 5.00 \\
\hline Traffic direction & $\mathrm{x}$ & & east bound \\
\hline
\end{tabular}

\section{Analysis parameters}

\begin{tabular}{|l|c|c|}
\hline Initial IRI & $\mathrm{x}$ & 63 \\
\hline Termal IRI (in/mile) & $\mathrm{x}$ & 172 \\
\hline AC surface down cracking - long cracking (ft/mi) & $\mathrm{x}$ & 1000 \\
\hline AC bottom up cracking - alligator cracking & $\mathrm{x}$ & 100 \\
\hline Chemically stabilized layer fatigue fracture (\%) & $\mathrm{x}$ & \\
\hline Permanent deformation - Total (in) & $\mathrm{x}$ & 100 \\
\hline Permanent deformation - AC only (in) & $\mathrm{x}$ & 0.75 \\
\hline
\end{tabular}

\section{B Inputs}

1 Traffic

\section{Initial two-day AADTT}

Two-away annual average daily traffic (AADT)

Percent of heavy vehicles (class 4 or higher)

Number of lanes in design direction

Percent of truck in design direction

Percent of truck in the design lane

Operational speed: $60 \mathrm{mph}$

Traffic growth: compound

\begin{tabular}{|c|c|c|}
\hline & $\mathrm{x}$ & $10000-40000$ \\
\hline $\mathrm{x}$ & $\mathrm{x}$ & $40-60$ \\
\hline & $\mathrm{x}$ & 2 \\
\hline & $\mathrm{x}$ & $70-60$ \\
\hline & $\mathrm{x}$ & $40-95$ \\
\hline & $\mathrm{x}$ & $1-70$ \\
\hline
\end{tabular}




\begin{tabular}{|c|c|c|c|}
\hline Traffic adjustment factors & FIX & VARY & Values/Ranges \\
\hline Monthly adjustment & $\mathrm{x}$ & & Default \\
\hline Vehicle class distribution & $\mathrm{x}$ & & Default \\
\hline Hourly distribution & $\mathrm{X}$ & & Default \\
\hline \multicolumn{4}{|l|}{ Axle load distribution } \\
\hline Axle type & $\mathrm{x}$ & & Default \\
\hline Single axle & $\mathrm{x}$ & & Default \\
\hline Tandem & $\mathrm{x}$ & & Default \\
\hline Tridem & $\mathrm{X}$ & & Default \\
\hline Quad & $\mathrm{X}$ & & Default \\
\hline \multicolumn{4}{|l|}{ General traffic inputs } \\
\hline Mean wheel location (inches from the lane marking) & & $\mathrm{X}$ & $5-36$ \\
\hline Traffic wander standard deviation (in) & & $\mathrm{x}$ & $7-13$ \\
\hline Design lane width $(\mathrm{ft})$ & $\mathrm{x}$ & & 12 \\
\hline Number axles truck & $\mathrm{x}$ & & Default \\
\hline \multicolumn{4}{|l|}{ Axle configuration } \\
\hline Average axle width $(\mathrm{ft})$ & & $\mathrm{x}$ & $8-10$ \\
\hline Dual tire spacing (in) & & $\mathrm{x}$ & $5-24$ \\
\hline Tire pressure (psi) & $\mathrm{x}$ & & 120 \\
\hline \multicolumn{4}{|l|}{ Axle spacing (in) } \\
\hline Tandem axle & $\mathrm{X}$ & & 51.6 \\
\hline Tridem axle & $\mathrm{X}$ & & 49.2 \\
\hline Quad axle & $\mathrm{x}$ & & 49.2 \\
\hline Wheelbase (This does not apply for flexible pavement) & & & \\
\hline
\end{tabular}

\section{Climate}

\begin{tabular}{|l|c|c|c|}
\hline Station Morgantown, WV & $\mathrm{x}$ & & Morgantown \\
\hline Latitude: 39.8 & $\mathrm{x}$ & & 39.8 \\
\hline Longitude: -79.55 & $\mathrm{x}$ & & -79.55 \\
\hline Elevation (ft) & $\mathrm{x}$ & & 1245 \\
\hline Depth of water table (ft) & $\mathrm{x}$ & & 20 \\
\hline
\end{tabular}

\section{Structure}

\begin{tabular}{|l|c|c|c|}
\hline HMA design procedure & & & \\
\hline Predictive model & & & \\
\hline $\begin{array}{l}\text { NCHRP 1-37A viscosity based model (nationally } \\
\text { calibrated) }\end{array}$ & $\mathrm{x}$ & & \\
\hline NCHRP 1-40D G* base model (nationally uncalibrated) & & & \\
\hline HMA rutting model coefficients & & & \\
\hline
\end{tabular}


Layer 1 (asphalt concrete layer) $9.5 \mathrm{~mm}$ mix

Surface short-wave absorptive

Asphalt material type

Layer thickness (in)

\begin{tabular}{|c|c|c|}
\hline FIX & VARY & Values/Ranges \\
\hline $\mathrm{X}$ & & 0.85 \\
\hline $\mathrm{X}$ & & $\begin{array}{c}\text { Asphalt } \\
\text { concrete }\end{array}$ \\
\hline $\mathrm{X}$ & & 2 \\
\hline
\end{tabular}

Asphalt mix

Cumulative $\%$ retained \#3/4 inch sieve

Cumulative $\%$ retained \#3/8 inch sieve

Cumulative $\%$ retained $\# 4$ inch sieve

Passing \#200 sieve

\begin{tabular}{|c|c|c}
\hline $\mathrm{X}$ & 0 \\
\hline $\mathrm{X}$ & & 7 \\
\hline $\mathrm{X}$ & & 43 \\
\hline $\mathrm{X}$ & & 4.5 \\
\hline
\end{tabular}

Asphalt binder

Superpave binder grading

Reference temperature $\left({ }^{\circ} \mathrm{F}\right)$

\begin{tabular}{c|c|c}
$\mathrm{x}$ & & $70-22$ \\
$\mathrm{x}$ & & 70 \\
\hline
\end{tabular}

Volumetric properties as built

Effective binder content

Air voids

Total unit weight (pcf)

Poisson's ratio: 0.35

Thermal properties

Thermal conductivity asphalt (BTU/hr-ft- ${ }^{\circ} \mathrm{F}: 0.67$

Heat capacity asphalt $\left(\mathrm{BTU} / \mathrm{lb}-{ }^{\circ} \mathrm{F}\right): 0.23$

\begin{tabular}{|c|c|c|}
\hline $\mathrm{X}$ & 7 \\
$\mathrm{X}$ & & 7 \\
\hline $\mathrm{X}$ & & 150 \\
\hline & & 0.35 \\
\hline $\mathrm{X}$ & & 0.67 \\
\hline $\mathrm{X}$ & & 0.23 \\
\hline
\end{tabular}

Layer 2 (asphalt concrete layer) $19 \mathrm{~mm}$ mix

Surface short-wave absorptive

Asphalt material type

Layer thickness (in)

\begin{tabular}{|c|c|c|}
\hline FIX & VARY & Values/Ranges \\
\hline $\mathrm{X}$ & & 0.85 \\
$\mathrm{X}$ & & $\begin{array}{c}\text { Asphalt } \\
\text { concrete }\end{array}$ \\
\hline $\mathrm{X}$ & & 3 \\
\hline
\end{tabular}

Asphalt mix

Cumulative $\%$ retained \#3/4 inch sieve

Cumulative $\%$ retained \#3/8 inch sieve

Cumulative $\%$ retained \#4 inch sieve

Passing \#200 sieve

Asphalt binder

Superpave binder grading

Reference temperature $\left({ }^{\circ} \mathrm{F}\right)$

\begin{tabular}{|c|c|c|}
\hline $\mathrm{X}$ & & 2 \\
\hline $\mathrm{X}$ & & 29 \\
\hline $\mathrm{X}$ & & 52 \\
\hline $\mathrm{X}$ & & 5.3 \\
\hline
\end{tabular}

Volumetric properties as built

Effective binder content

Air voids

\begin{tabular}{|c|c|c|}
\hline $\mathrm{x}$ & & $70-22$ \\
\hline $\mathrm{X}$ & & 70 \\
\hline
\end{tabular}




\begin{tabular}{|l|c|c|c|}
\hline Total unit weight (pcf) & $\mathrm{x}$ & & 150 \\
\hline Poisson's ratio: 0.35 & $\mathrm{x}$ & & 0.35 \\
\hline Thermal properties & $\mathrm{x}$ & & 0.67 \\
\hline Thermal conductivity asphalt (BTU/hr-ft- ${ }^{\circ} \mathrm{F}: 0.67$ & $\mathrm{x}$ & & 0.23 \\
\hline Heat capacity asphalt (BTU/lb- $\left.{ }^{\circ} \mathrm{F}\right): 0.23$ & \\
\hline
\end{tabular}

\begin{tabular}{|c|c|c|c|}
\hline Layer 3 (asphalt concrete layer) $37 \mathrm{~mm}$ mix & FIX & VARY & Values/Ranges \\
\hline Surface short-wave absorptive & $\mathrm{x}$ & & 0.85 \\
\hline Asphalt material type & $\mathrm{x}$ & & $\begin{array}{l}\text { Asphalt } \\
\text { concrete }\end{array}$ \\
\hline Layer thickness (in) & $\mathrm{x}$ & & 10 \\
\hline \multicolumn{4}{|l|}{ Asphalt mix } \\
\hline Cumulative $\%$ retained $\# 3 / 4$ inch sieve & $\mathrm{x}$ & & 33 \\
\hline Cumulative $\%$ retained $\# 3 / 8$ inch sieve & $\mathrm{x}$ & & 55 \\
\hline Cumulative \% retained \#4 inch sieve & $\mathrm{x}$ & & 64 \\
\hline Passing \#200 sieve & $\mathrm{x}$ & & 3.6 \\
\hline \multicolumn{4}{|l|}{ Asphalt binder } \\
\hline Superpave binder grading & $\mathrm{x}$ & & $70-22$ \\
\hline Reference temperature $\left({ }^{\circ} \mathrm{F}\right)$ & $\mathrm{x}$ & & 70 \\
\hline \multicolumn{4}{|l|}{ Volumetric properties as built } \\
\hline Effective binder content & $\mathrm{x}$ & & 5 \\
\hline Air voids & $\mathrm{x}$ & & 6 \\
\hline Total unit weight (pcf) & $\mathrm{x}$ & & 150 \\
\hline Poisson's ratio: 0.35 & $\mathrm{x}$ & & 0.35 \\
\hline \multicolumn{4}{|l|}{ Thermal properties } \\
\hline Thermal conductivity asphalt (BTU/hr-ft- ${ }^{\circ} \mathrm{F}: 0.67$ & $\mathrm{x}$ & & 0.67 \\
\hline Heat capacity asphalt $\left(\mathrm{BTU} / \mathrm{lb}-{ }^{\circ} \mathrm{F}\right): 0.23$ & $\mathrm{x}$ & & 0.23 \\
\hline
\end{tabular}

\begin{tabular}{|c|c|c|c|}
\hline Layer 4 (asphalt treated permeable base) & FIX & VARY & Values/Ranges \\
\hline Surface short-wave absorptive & $\mathrm{x}$ & & 0.85 \\
\hline Asphalt material type & $\mathrm{x}$ & & $\begin{array}{l}\text { Asphalt } \\
\text { concrete }\end{array}$ \\
\hline Layer thickness (in) & $\mathrm{x}$ & & 3 \\
\hline \multicolumn{4}{|l|}{ Asphalt mix } \\
\hline Cumulative $\%$ retained \#3/4 inch sieve & $\mathrm{x}$ & & 30 \\
\hline Cumulative $\%$ retained $\# 3 / 8$ inch sieve & $\mathrm{x}$ & & 80 \\
\hline Cumulative $\%$ retained $\# 4$ inch sieve & $\mathrm{x}$ & & 85 \\
\hline Passing \#200 sieve & $\mathrm{x}$ & & 2 \\
\hline
\end{tabular}




\begin{tabular}{|l|c|c|c|}
\hline Asphalt binder & FIX & VARY & Values/Ranges \\
\hline Superpave binder grading & $\mathrm{x}$ & & $70-22$ \\
\hline Reference temperature $\left({ }^{\circ} \mathrm{F}\right)$ & $\mathrm{x}$ & & 70 \\
\hline Volumetric properties as built & $\mathrm{x}$ & & 4 \\
\hline Effective binder content & $\mathrm{x}$ & & 6 \\
\hline Air voids & $\mathrm{x}$ & & 150 \\
\hline Total unit weight (pcf) & $\mathrm{x}$ & & 0.35 \\
\hline Poisson's ratio: 0.35 & $\mathrm{x}$ & & 0.67 \\
\hline Thermal properties & $\mathrm{x}$ & & 0.23 \\
\hline Thermal conductivity asphalt $\left(\mathrm{BTU} / \mathrm{hr}-\mathrm{ft}-{ }^{\circ} \mathrm{F}: 0.67\right.$ & & \\
\hline Heat capacity asphalt $\left(\mathrm{BTU} / \mathrm{lb}^{-}{ }^{\mathrm{0}} \mathrm{F}\right): 0.23$ &
\end{tabular}

\section{Layer 5 (Subgrade)}

\begin{tabular}{|c|c|c|}
\hline Unbound material (classification) & $\mathrm{x}$ & $\mathrm{MH}$ \\
\hline Poisson's ratio: 0.35 & $\mathrm{x}$ & 0.35 \\
\hline Coefficient of lateral pressure $K_{o}$ & $\mathrm{x}$ & 0.5 \\
\hline Modulus (psi) & $\mathrm{x}$ & 15000 \\
\hline \multicolumn{3}{|c|}{ ICM (Climate model input for this layer's material) } \\
\hline Gradation & $\mathrm{x}$ & \\
\hline Plasticity index $(\mathrm{PI})$ & $\mathrm{x}$ & 5 \\
\hline Liquid limit (LL) & $\mathrm{x}$ & 45 \\
\hline Compact layer & $\mathrm{x}$ & yes \\
\hline \multicolumn{3}{|c|}{ Index properties from sieve analysis } \\
\hline$\%$ passing \# 200 & $\mathrm{x}$ & 54.3 \\
\hline$\%$ passing \#40 & $\mathrm{x}$ & 0 \\
\hline$\%$ passing \#4 & $\mathrm{x}$ & 86.9 \\
\hline $\mathrm{D} 10(\mathrm{~mm})$ & $\mathrm{x}$ & 0.0003384 \\
\hline $\mathrm{D} 20(\mathrm{~mm})$ & $\mathrm{x}$ & 0.001145 \\
\hline D30(mm) & $\mathrm{x}$ & 0.003876 \\
\hline $\mathrm{D} 60(\mathrm{~mm})$ & $\mathrm{x}$ & 0.1234 \\
\hline D90(mm) & $\mathrm{x}$ & 9.109 \\
\hline
\end{tabular}

\section{User overridable}

Index properties

\begin{tabular}{|l|c|c|c|}
\hline Maximum dry unit weight (PCF) & $\mathrm{x}$ & 119.2 \\
\hline Specific gravity & $\mathrm{x}$ & 2.7 \\
\hline Sat hydraulic conductivity (ft/hr) & $\mathrm{x}$ & & $9.3 \mathrm{e}-0.007$ \\
\hline Optimum gravimetric water content (\%) & $\mathrm{x}$ & & 11.4 \\
\hline Degree of saturation of optimum \% & $\mathrm{x}$ & & 74.4 \\
\hline
\end{tabular}




\begin{tabular}{|l|c|c|c|}
\cline { 2 - 3 } \multicolumn{1}{l|}{ Overridable soil water characteristic curve: } & FIX & VARY & Values/Ranges \\
\hline Af: 65.23 & $\mathrm{x}$ & & 65.23 \\
\hline Bf: 1.034 & $\mathrm{x}$ & & 1.034 \\
\hline Cf: 0.4994 & $\mathrm{x}$ & & 0.4994 \\
\hline Hf: 500 & $\mathrm{x}$ & & 500 \\
\hline
\end{tabular}

\section{Thermal cracking}

\begin{tabular}{|c|c|c|}
\hline Average tensile strength at $14 \mathrm{oF}$ (PSI): & $\mathrm{X}$ & 727 \\
\hline Mixture VMA (\%): 18.6 & & NA \\
\hline Agg coefficient of thermal contraction: $1 \mathrm{e}-006$ & & NA \\
\hline Mix coefficient of thermal contraction & $\mathrm{x}$ & $2.2 \times 10^{\wedge}-05$ \\
\hline
\end{tabular}




\section{MATERIAL SENSITIVITY - STRUCTURE 1 MATERIAL PROPERTIES AND RANGES}

\section{A. Project description}

1 General information FIX VARY Values/Ranges

Design life (year)

Base/subgrade construction month

Pavement construction month

Traffic open

Type design: flexible pavement

\begin{tabular}{|c|c|c|}
\hline $\mathrm{x}$ & & 20 \\
\hline $\mathrm{X}$ & & May \\
\hline $\mathrm{x}$ & & June \\
\hline $\mathrm{x}$ & & July \\
\hline $\mathrm{x}$ & & Flexible \\
\hline
\end{tabular}

2 Site project identification

\begin{tabular}{|l|c|c|c|}
\hline Location & $\mathrm{x}$ & & Morgantown \\
\hline Station/milepost format: miles & $\mathrm{x}$ & & 0.00 \\
\hline Station/milepost begin: & $\mathrm{x}$ & & 0.20 \\
\hline Station/milepost end: & $\mathrm{x}$ & & 5.00 \\
\hline Traffic direction & $\mathrm{x}$ & & east bound \\
\hline
\end{tabular}

3 Analysis parameters

\begin{tabular}{|l|c|c|c|}
\hline Initial IRI & $\mathrm{x}$ & & \\
\hline Termal IRI (in/mile) & $\mathrm{x}$ & & 172 \\
\hline AC surface down cracking - long cracking (ft/mi) & $\mathrm{x}$ & & 1000 \\
\hline AC bottom up cracking - alligator cracking & $\mathrm{x}$ & & 100 \\
\hline Chemically stabilized layer fatigue fracture (\%) & $\mathrm{x}$ & & 100 \\
\hline Permanent deformation - Total (in) & $\mathrm{x}$ & & 0.75 \\
\hline Permanent deformation - AC only (in) & $\mathrm{x}$ & & 0.25 \\
\hline
\end{tabular}

\section{B Inputs}

1 Traffic

\section{Initial two-day AADTT}

Two-away annual average daily traffic (AADT)

Percent of heavy vehicles (class 4 or higher)

Number of lanes in design direction

Percent of trucks in design direction

Percent of truck in the design line

Operational speed: $60 \mathrm{mph}$

Traffic growth: compound

\begin{tabular}{|c|c|c|}
\hline$x$ & & 25000 \\
\hline$x$ & & 50 \\
\hline$x$ & & 2 \\
\hline$x$ & & 50 \\
\hline$x$ & & 95 \\
\hline$x$ & & 60 \\
\hline
\end{tabular}




\begin{tabular}{|c|c|c|c|}
\hline Traffic adjustment factors & FIX & VARY & Values/Ranges \\
\hline Monthly adjustment & $\mathrm{X}$ & & Default \\
\hline Vehicle class distribution & $\mathrm{x}$ & & Default \\
\hline Hourly distribution & $\mathrm{x}$ & & Default \\
\hline \multicolumn{4}{|l|}{ Axle load distribution } \\
\hline Axle type & $\mathrm{x}$ & & Default \\
\hline Single axle & $\mathrm{x}$ & & Default \\
\hline Tandem & $\mathrm{x}$ & & Default \\
\hline Tridem & $\mathrm{x}$ & & Default \\
\hline Quad & $\mathrm{x}$ & & Default \\
\hline \multicolumn{4}{|l|}{ General traffic inputs } \\
\hline Mean wheel location (inches from the lane marking) & $\mathrm{x}$ & & 18 \\
\hline Traffic wander standard deviation (in) & $\mathrm{X}$ & & 10 \\
\hline Design lane width $(\mathrm{ft})$ & $\mathrm{X}$ & & 12 \\
\hline Number axles truck & $\mathrm{X}$ & & Default \\
\hline \multicolumn{4}{|l|}{ Axle configuration } \\
\hline Average axle width $(\mathrm{ft})$ & $\mathrm{x}$ & & 8.5 \\
\hline Dual tire spacing (in) & $\mathrm{x}$ & & 12 \\
\hline Tire pressure $(\mathrm{psi})$ & $\mathrm{x}$ & & 120 \\
\hline \multicolumn{4}{|l|}{ Axle spacing (in) } \\
\hline Tandem axle & $\mathrm{x}$ & & 51.6 \\
\hline Tridem axle & $\mathrm{x}$ & & 49.2 \\
\hline Quad axle & $\mathrm{x}$ & & 49.2 \\
\hline Wheelbase (This does not apply for flexible pavement) & & & \\
\hline
\end{tabular}

2 Climate

\begin{tabular}{|c|c|c|}
\hline Station Morgantown, WV & $\mathrm{x}$ & Morgantown \\
\hline Latitude: 39.8 & $\mathrm{x}$ & 39.8 \\
\hline Longitude: -79.55 & $\mathrm{x}$ & -79.55 \\
\hline Elevation $(\mathrm{ft})$ & $\mathrm{x}$ & 1245 \\
\hline Depth of water table $(\mathrm{ft})$ & $\mathrm{X}$ & 20 \\
\hline
\end{tabular}

\section{Structure}

\begin{tabular}{|c|c|c|}
\hline HMA design procedure & & \\
\hline Predictive model & & \\
\hline $\begin{array}{l}\text { NCHRP 1-37A viscosity based model (nationally } \\
\text { calibrated) }\end{array}$ & $\mathrm{X}$ & $\checkmark$ \\
\hline NCHRP 1-40D G* base model (nationally uncalibrated) & & \\
\hline
\end{tabular}




\begin{tabular}{|l|c|c|c|}
\hline HMA rutting model coefficients & FIX & VARY & Values/Ranges \\
\hline NCHRP 1-37A coefficient & X & & $\checkmark$ \\
\hline
\end{tabular}

Layer 1 (asphalt concrete layer) $9.5 \mathrm{~mm}$ mix

\begin{tabular}{|c|c|c|c|}
\hline Surface short-wave absorptive & & $\mathrm{x}$ & $0.8-0.90$ \\
\hline Asphalt material type & $\mathrm{x}$ & & $\begin{array}{l}\text { Asphalt } \\
\text { concrete }\end{array}$ \\
\hline Layer thickness (in) & $\mathrm{x}$ & & 2 \\
\hline \multicolumn{4}{|l|}{ Asphalt mix } \\
\hline Cumulative $\%$ retained $\# 3 / 4$ inch sieve & & $\mathrm{x}$ & 0 \\
\hline Cumulative $\%$ retained $\# 3 / 8$ inch sieve & & $\mathrm{x}$ & 7 \\
\hline Cumulative $\%$ retained $\# 4$ inch sieve & & $\mathrm{x}$ & 43 \\
\hline Passing \#200 sieve & & $\mathrm{x}$ & 4.5 \\
\hline \multicolumn{4}{|l|}{ Asphalt binder } \\
\hline Superpave binder grading & $\mathrm{x}$ & & $70-22$ \\
\hline Reference temperature $\left({ }^{\circ} \mathrm{F}\right)$ & $\mathrm{x}$ & & 70 \\
\hline \multicolumn{4}{|l|}{ Volumetric properties as built } \\
\hline Effective binder content & & $\mathrm{x}$ & $4.5-6.5$ \\
\hline Air voids & & $\mathrm{x}$ & $3-10$ \\
\hline Total unit weight (pcf) & & $\mathrm{x}$ & $145-150$ \\
\hline Poisson's ratio: 0.35 & & $\mathrm{x}$ & $0.25-0.40$ \\
\hline \multicolumn{4}{|l|}{ Thermal properties } \\
\hline Thermal conductivity asphalt (BTU/hr-ft- ${ }^{\circ} \mathrm{F}: 0.67$ & & $\mathrm{x}$ & $0.5-0.8$ \\
\hline Heat capacity asphalt $\left(\mathrm{BTU} / \mathrm{lb}-{ }^{\circ} \mathrm{F}\right): 0.23$ & & $\mathrm{x}$ & $0.22-0.5$ \\
\hline
\end{tabular}

Layer 2 (asphalt concrete layer) $19 \mathrm{~mm}$ mix

Surface short-wave absorptive

Asphalt material type

Layer thickness (in)

Asphalt mix

Cumulative $\%$ retained \#3/4 inch sieve

Cumulative $\%$ retained $\# 3 / 8$ inch sieve

Cumulative $\%$ retained \#4 inch sieve

Passing \#200 sieve

\begin{tabular}{|c|c|c|} 
& $\mathrm{x}$ & $0.8-0.90$ \\
$\mathrm{X}$ & & Asphalt concrete \\
\hline $\mathrm{X}$ & & 3 \\
\hline
\end{tabular}

Asphalt binder

Superpave binder grading

Reference temperature $\left({ }^{\circ} \mathrm{F}\right)$

\begin{tabular}{|c|c|c|}
\hline $\mathrm{x}$ & & 2 \\
\hline $\mathrm{x}$ & & 29 \\
\hline $\mathrm{x}$ & & 52 \\
$\mathrm{x}$ & & 5.3 \\
\hline
\end{tabular}

\begin{tabular}{|c|c|c|}
\hline $\mathrm{x}$ & & $70-22$ \\
\hline $\mathrm{x}$ & & 70 \\
\hline
\end{tabular}




\begin{tabular}{|l|c|c|c|}
\hline Volumetric properties as built & FIX & VARY & Values/Ranges \\
\hline Effective binder content & & $\mathrm{x}$ & $3.5-6$ \\
\hline Air voids & & $\mathrm{x}$ & $3-10$ \\
\hline Total unit weight (pcf) & & $\mathrm{x}$ & $145-150$ \\
\hline Poisson's ratio: 0.35 & & $\mathrm{x}$ & $0.25-0.40$ \\
\hline Thermal properties & & & \\
\hline Thermal conductivity asphalt $\left(\mathrm{BTU} / \mathrm{hr}-\mathrm{ft}-{ }^{\circ} \mathrm{F}: 0.67\right.$ & & $\mathrm{x}$ & $0.5-0.8$ \\
\hline Heat capacity asphalt $\left(\mathrm{BTU} / \mathrm{lb}-{ }^{\mathrm{O}} \mathrm{F}\right): 0.23$ & & & $0.22-0.5$ \\
\hline
\end{tabular}

\section{Layer 3 (asphalt concrete layer) $37 \mathrm{~mm}$ mix}

Surface short-wave absorptive

Asphalt material type

Layer thickness (in)

\begin{tabular}{|c|c|c|c|} 
& & $\mathrm{x}$ & $0.8-0.90$ \\
& $\mathrm{x}$ & & Asphalt concrete \\
\hline & $\mathrm{x}$ & & 10 \\
\hline
\end{tabular}

Asphalt mix

Cumulative \% retained \#3/4 inch sieve

Cumulative $\%$ retained \#3/8 inch sieve

Cumulative $\%$ retained \#4 inch sieve

Passing \#200 sieve

\begin{tabular}{|c|c|c|}
\hline $\mathrm{x}$ & & 33 \\
\hline $\mathrm{x}$ & & 55 \\
\hline $\mathrm{x}$ & & 64 \\
\hline $\mathrm{x}$ & & 3.6 \\
\hline
\end{tabular}

Asphalt binder

Superpave binder grading

Reference temperature $\left({ }^{\circ} \mathrm{F}\right)$

\begin{tabular}{c|c|c|}
$\mathrm{X}$ & & $70-22$ \\
$\mathrm{x}$ & & 70 \\
\hline
\end{tabular}

\section{Volumetric properties as built}

Effective binder content

Air voids

Total unit weight (pcf)

Poisson's ratio: 0.35

Thermal properties

Thermal conductivity asphalt (BTU/hr-ft- ${ }^{\circ} \mathrm{F}: 0.67$

Heat capacity asphalt (BTU/lb- ${ }^{\circ}$ F): 0.23

\begin{tabular}{|l|l|}
$\mathrm{x}$ \\
$\mathrm{x}$
\end{tabular}

\begin{tabular}{|l|c|c|c|}
\hline & & $\mathrm{x}$ & $2.5-4.0$ \\
\hline & & $\mathrm{x}$ & $6-12$ \\
\hline & & $\mathrm{x}$ & $145-150$ \\
\hline & & $\mathrm{x}$ & $0.25-0.40$ \\
\hline & & & \\
\hline
\end{tabular}

Layer 4 (asphalt treated permeable base)

\begin{tabular}{|c|c|c|c|}
\hline Surface short-wave absorptive & & $\mathrm{X}$ & $0.8-0.90$ \\
\hline Asphalt material type & $\mathrm{x}$ & & Asphalt concrete \\
\hline Layer thickness (in) & $\mathrm{x}$ & & 3 \\
\hline \multicolumn{4}{|l|}{ Asphalt mix } \\
\hline Cumulative $\%$ retained $\# 3 / 4$ inch sieve & & $\mathrm{X}$ & 30 \\
\hline Cumulative $\%$ retained $\# 3 / 8$ inch sieve & & $\mathrm{x}$ & 80 \\
\hline Cumulative $\%$ retained \#4 inch sieve & & $\mathrm{x}$ & 85 \\
\hline Passing \#200 sieve & & $\mathrm{x}$ & 2 \\
\hline
\end{tabular}




\begin{tabular}{|l|c|c|c|}
\hline Asphalt binder & FIX & VARY & Values/Ranges \\
\hline Superpave binder grading & $\mathrm{x}$ & & $70-22$ \\
\hline Reference temperature $\left({ }^{\circ} \mathrm{F}\right)$ & $\mathrm{x}$ & & 70 \\
\hline Volumetric properties as built & & $\mathrm{x}$ & $2-3$ \\
\hline Effective binder content & & $\mathrm{x}$ & $15-20$ \\
\hline Air voids & & $\mathrm{x}$ & $145-150$ \\
\hline Total unit weight $(\mathrm{pcf})$ & & $\mathrm{x}$ & $0.25-0.40$ \\
\hline Poisson's ratio: 0.35 & & & \\
\hline Thermal properties & & $\mathrm{x}$ & $0.5-1.0$ \\
\hline Thermal conductivity asphalt (BTU/hr-ft-oF: 0.67 & & $\mathrm{x}$ & $0.1-0.5$ \\
\hline Heat capacity asphalt (BTU/lb-oF): 0.23 & & \\
\hline
\end{tabular}

\section{Layer 5 (Subgrade)}

Unbound material (classification):

Poisson's ratio: 0.35

Coefficient of lateral pressure $\mathrm{K}_{\mathrm{o}}: 0.5$

Modulus (psi)

ICM (Climate model input for this layer's material)

\section{Gradation}

Plasticity index (PI)

Liquid limit (LL)

Compact layer

\section{Index properties from sieve analysis}

$\%$ passing \# 200

$\%$ passing \#40

$\%$ passing \#4

D10 (mm)

D20

D30

D60

D90

\begin{tabular}{c|c|c|}
$\mathrm{x}$ & & $\mathrm{MH}$ \\
& $\mathrm{x}$ & $0.3-0.45$ \\
& $\mathrm{x}$ & $0.5-0.70$ \\
& $\mathrm{x}$ & $5000-9000$
\end{tabular}

\begin{tabular}{|c|c|c|}
\hline $\mathrm{X}$ & & \\
\hline $\mathrm{X}$ & & 5 \\
\hline $\mathrm{X}$ & & 45 \\
\hline $\mathrm{X}$ & & yes \\
\hline
\end{tabular}

\begin{tabular}{|c|c|c|}
\hline $\mathrm{X}$ & & 54.3 \\
$\mathrm{X}$ & & 0 \\
\hline $\mathrm{X}$ & & 86.9 \\
\hline $\mathrm{X}$ & & 0.0003384 \\
\hline $\mathrm{X}$ & & 0.001145 \\
\hline $\mathrm{X}$ & & 0.003876 \\
\hline $\mathrm{X}$ & & 0.1234 \\
\hline
\end{tabular}

User overridable

Maximum dry unit weight (PCF)

Specific gravity

Sat hydraulic conductivity (ft/hr)

Optimum gravimetric water content $(\%)$

Degree of saturation of optimum $\%$

\begin{tabular}{|c|c|c|}
\multicolumn{2}{|c}{ index properties } \\
\hline $\mathrm{X}$ & & 119.2 \\
$\mathrm{X}$ & & 2.7 \\
\hline $\mathrm{X}$ & & $9.3 \mathrm{e}-0.007$ \\
\hline $\mathrm{X}$ & & 11.4 \\
\hline $\mathrm{X}$ & & 74.4 \\
\hline
\end{tabular}




\begin{tabular}{|l|c|c|c|}
\cline { 2 - 4 } \multicolumn{1}{l|}{ Overridable soil water characteristic curve: } & FIX & VARY & Values/Ranges \\
\hline Af: 65.23 & $\mathrm{x}$ & & 65.23 \\
\hline Bf: 1.034 & $\mathrm{x}$ & & 1.034 \\
\hline Cf: 0.4994 & $\mathrm{x}$ & & 0.4994 \\
\hline Hf: 500 & $\mathrm{x}$ & & 500 \\
\hline
\end{tabular}

Thermal cracking

Average tensile strength at 14oF (PSI): 375.74

Mixture VMA (\%): 18.6

Agg coefficient of thermal contraction: 1e-006

Mix coefficient of thermal contraction

$\mathrm{X}$

$500-1500$

NA

NA

$\mathrm{X} \quad(2.2-3.4) \mathrm{x} 10^{\wedge}-05$


TRAFFIC SENSITIVITY - STRUCTURE 2

MATERIAL PROPERTIES AND RANGES

\section{A. Project description}

1 General information FIX VARY Values/Ranges

Design life (year)

Base/subgrade construction month

Pavement construction month

Traffic open

Type design: flexible pavement

\begin{tabular}{|c|c|c|}
\hline $\mathrm{X}$ & & 20 \\
\hline $\mathrm{X}$ & & May \\
\hline $\mathrm{X}$ & & June \\
\hline $\mathrm{X}$ & & July \\
\hline $\mathrm{X}$ & & Flexible \\
\hline
\end{tabular}

2 Site project identification

\begin{tabular}{|l|c|c|c|}
\hline Location & $\mathrm{x}$ & & Morgantown \\
\hline Station/milepost format: miles & $\mathrm{x}$ & & 0.00 \\
\hline Station/milepost begin: & $\mathrm{x}$ & & 0.20 \\
\hline Station/milepost end: & $\mathrm{x}$ & & 5.00 \\
\hline Traffic direction & $\mathrm{x}$ & & east bound \\
\hline
\end{tabular}

\section{Analysis parameters}

\begin{tabular}{|c|c|c|}
\hline Initial IRI & $\mathrm{x}$ & \\
\hline Termal IRI (in/mile) & $\mathrm{x}$ & 172 \\
\hline AC surface down cracking - long cracking $(\mathrm{ft} / \mathrm{mi})$ & $\mathrm{x}$ & 1000 \\
\hline AC bottom up cracking - alligator cracking & $\mathrm{x}$ & 100 \\
\hline Chemically stabilized layer fatigue fracture (\%) & $\mathrm{x}$ & 100 \\
\hline Permanent deformation - Total (in) & $\mathrm{x}$ & 0.75 \\
\hline Permanent deformation - AC only (in) & $\mathrm{x}$ & 0.25 \\
\hline
\end{tabular}

\section{B Inputs}

1 Traffic

\begin{tabular}{|l|c|c|c|}
\hline Initial two-day AADTT & & $\mathrm{x}$ & $1000-4000$ \\
\hline Two-away annual average daily traffic (AADT) & & $\mathrm{x}$ & $40-60$ \\
\hline Percent of heavy vehicles (class 4 or higher) & $\mathrm{x}$ & & 2 \\
\hline Number of lanes in design direction & & $\mathrm{x}$ & $40-60$ \\
\hline Percent of trucks in design direction & & $\mathrm{x}$ & $70-95$ \\
\hline Percent of trucks in the design line & & $\mathrm{x}$ & $40-70$ \\
\hline Operational speed: 60 mph & & $\mathrm{x}$ & $1-8$ \\
\hline Traffic growth: compound &
\end{tabular}




\begin{tabular}{|c|c|c|c|}
\hline Traffic adjustment factors & FIX & VARY & Values/Ranges \\
\hline Monthly adjustment (table) & $\mathrm{x}$ & & Default \\
\hline Vehicle class distribution (Use level 3) & $\mathrm{x}$ & & Default \\
\hline Hourly distribution (table) & $\mathrm{x}$ & & Default \\
\hline \multicolumn{4}{|l|}{ Axle load distribution } \\
\hline Axle type & $\mathrm{x}$ & & Default \\
\hline Single axle & $\mathrm{x}$ & & Default \\
\hline Tandem & $\mathrm{x}$ & & Default \\
\hline Tridem & $\mathrm{x}$ & & Default \\
\hline Quad & $\mathrm{x}$ & & Default \\
\hline \multicolumn{4}{|l|}{ General traffic inputs } \\
\hline Mean wheel location (inches from the lane marking) & & $\mathrm{x}$ & $5-36$ \\
\hline Traffic wander standard deviation (in) & & $\mathrm{x}$ & $7-13$ \\
\hline Design lane width $(\mathrm{ft})$ & $\mathrm{x}$ & & 12 \\
\hline Number axles truck & $\mathrm{x}$ & & Default \\
\hline \multicolumn{4}{|l|}{ Axle configuration } \\
\hline Average axle width $(\mathrm{ft})$ & & $\mathrm{x}$ & $8-10$ \\
\hline Dual tire spacing (in) & & $\mathrm{x}$ & $5-24$ \\
\hline Tire pressure (psi) & $\mathrm{x}$ & & 120 \\
\hline \multicolumn{4}{|l|}{ Axle spacing (in) } \\
\hline Tandem axle & $\mathrm{x}$ & & 51.6 \\
\hline Tridem axle & $\mathrm{x}$ & & 49.2 \\
\hline Quad axle & $\mathrm{x}$ & & 49.2 \\
\hline Wheelbase (This does not apply for flexible pavement) & & & \\
\hline
\end{tabular}

\section{Climate}

\begin{tabular}{|c|c|c|}
\hline Station Morgantown, WV & $\mathrm{x}$ & Morgantown \\
\hline Latitude: 39.8 & $\mathrm{x}$ & 39.8 \\
\hline Longitude: -79.55 & $\mathrm{x}$ & -79.55 \\
\hline Elevation $(\mathrm{ft})$ & $\mathrm{x}$ & 1245 \\
\hline Depth of water table (ft) & $\mathrm{x}$ & 20 \\
\hline
\end{tabular}

\section{Structure}

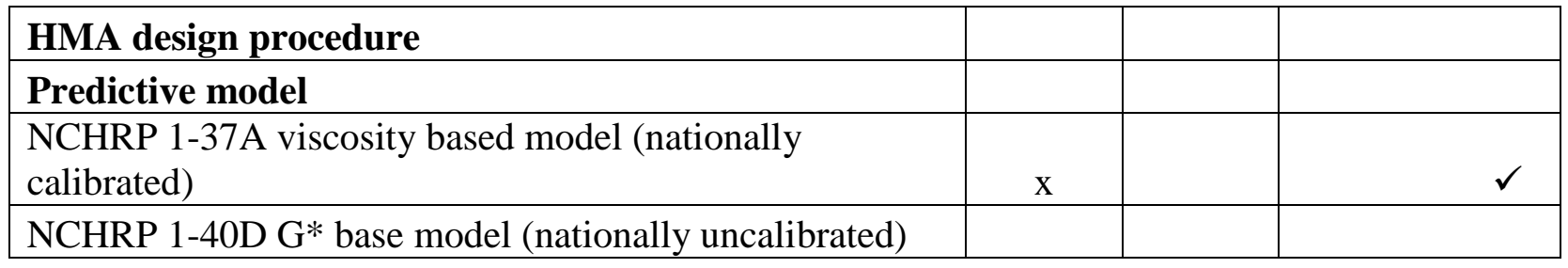


HMA rutting model coefficients

NCHRP 1-37A coefficient

\begin{tabular}{|c|c|c|}
\hline FIX & VARY & Values/Ranges \\
\hline $\mathrm{X}$ & & $\checkmark$ \\
\hline
\end{tabular}

Layer 1 (asphalt concrete layer) $9.5 \mathrm{~mm}$ mix

\begin{tabular}{|c|c|c|c|}
\hline Surface short-wave absorptive & & $\mathrm{x}$ & 0.85 \\
\hline Asphalt material type & $\mathrm{x}$ & & $\begin{array}{l}\text { Asphalt } \\
\text { concrete }\end{array}$ \\
\hline Layer thickness (in) & $\mathrm{x}$ & & 4 \\
\hline \multicolumn{4}{|l|}{ Asphalt mix } \\
\hline Cumulative $\%$ retained $\# 3 / 4$ inch sieve & $\mathrm{x}$ & & 0 \\
\hline Cumulative $\%$ retained $\# 3 / 8$ inch sieve & $\mathrm{x}$ & & 7 \\
\hline Cumulative $\%$ retained \#4 inch sieve & $\mathrm{x}$ & & 43 \\
\hline Passing \#200 sieve & $\mathrm{x}$ & & 4.5 \\
\hline \multicolumn{4}{|l|}{ Asphalt binder } \\
\hline Superpave binder grading & $\mathrm{x}$ & & $70-22$ \\
\hline Reference temperature $\left({ }^{\circ} \mathrm{F}\right)$ & $\mathrm{x}$ & & 70 \\
\hline \multicolumn{4}{|l|}{ Volumetric properties as built } \\
\hline Effective binder content & $\mathrm{x}$ & $\mathrm{x}$ & 7 \\
\hline Air voids & $\mathrm{x}$ & $\mathrm{x}$ & 7 \\
\hline Total unit weight (pcf) & $\mathrm{x}$ & $\mathrm{x}$ & 150 \\
\hline Poisson's ratio: 0.35 & $\mathrm{x}$ & $\mathrm{x}$ & 0.35 \\
\hline \multicolumn{4}{|l|}{ Thermal properties } \\
\hline Thermal conductivity asphalt (BTU/hr-ft- ${ }^{\circ} \mathrm{F}: 0.67$ & $\mathrm{x}$ & $\mathrm{x}$ & 0.67 \\
\hline Heat capacity asphalt (BTU/lb- $\left.{ }^{\circ} \mathrm{F}\right): 0.23$ & $\mathrm{x}$ & $\mathrm{x}$ & 0.23 \\
\hline
\end{tabular}

Layer 2 (Unbound Base)

\begin{tabular}{|l|c|c|c|}
\hline Unbound material (classification) & $\mathrm{x}$ & & Crushed Stone \\
\hline Poisson's ratio: & $\mathrm{x}$ & & 0.35 \\
\hline Coefficient of lateral pressure $\mathrm{K}_{\mathrm{o}}: 0.5$ & $\mathrm{x}$ & & 0.5 \\
\hline Modulus (psi) & $\mathrm{x}$ & & 30000 \\
\hline ICM (Climate model input for this layer's material) & $\mathrm{x}$ & & \\
\hline Gradation & $\mathrm{x}$ & & 1 \\
\hline Plasticity index (PI) & $\mathrm{x}$ & & 6 \\
\hline Liquid limit (LL) & $\mathrm{x}$ & & yes \\
\hline Compact layer & &
\end{tabular}




\begin{tabular}{|c|c|c|c|}
\hline Index properties from sieve analysis & FIX & VARY & Values/Ranges \\
\hline$\%$ passing \# 200 & $\mathrm{x}$ & & 8.7 \\
\hline$\%$ passing \#40 & $\mathrm{x}$ & & 20 \\
\hline$\%$ passing \#4 & $\mathrm{x}$ & & 44.7 \\
\hline $\mathrm{D} 10(\mathrm{~mm})$ & $\mathrm{x}$ & & 0.1035 \\
\hline D20 & $\mathrm{x}$ & & 0.425 \\
\hline D30 & $\mathrm{x}$ & & 1.306 \\
\hline D60 & $\mathrm{x}$ & & 10.82 \\
\hline D90 & $\mathrm{x}$ & & 46.19 \\
\hline \multicolumn{3}{|l|}{ User overridable } & index properties \\
\hline Maximum dry unit weight (PCF) & $\mathrm{x}$ & & 127.2 \\
\hline Specific gravity & $\mathrm{x}$ & & 2.7 \\
\hline Sat hydraulic conductivity (ft/hr) & $\mathrm{x}$ & & 0.051 \\
\hline Optimum gravimetric water content $(\%)$ & $\mathrm{x}$ & & 7.4 \\
\hline Degree of saturation of optimum \% & $\mathrm{x}$ & & 61.2 \\
\hline \multicolumn{4}{|c|}{ Overridable soil water characteristic curve: } \\
\hline Af: 65.23 & $\mathrm{x}$ & & 7.255 \\
\hline Bf: 1.034 & $\mathrm{x}$ & & 1.333 \\
\hline Cf: 0.4994 & $\mathrm{x}$ & & 0.8242 \\
\hline Hf: 500 & $\mathrm{x}$ & & 117.4 \\
\hline
\end{tabular}

Layer 3 (unbound Sub-Base)

\begin{tabular}{|l|c|c|c|}
\hline Unbound material (classification): Crushed Gavel & $\mathrm{x}$ & & \\
\hline Poisson's ratio: 0.35 & & $\mathrm{x}$ & 0.35 \\
\hline Coefficient of lateral pressure $\mathrm{K}_{\mathrm{O}}: 0.5$ & & $\mathrm{x}$ & 0.5 \\
\hline Modulus (psi) & & $\mathrm{x}$ & 25000 \\
\hline ICM (Climate model input for this layer's material) & $\mathrm{x}$ & & \\
\hline Gradation & $\mathrm{x}$ & & \multicolumn{2}{|c|}{1} \\
\hline Plasticity index (PI) & $\mathrm{x}$ & & 6 \\
\hline Liquid limit (LL) & $\mathrm{x}$ & & yes \\
\hline Compact layer & & \\
\hline
\end{tabular}




\begin{tabular}{|c|c|c|c|}
\hline Index properties from sieve analysis & FIX & VARY & Values/Ranges \\
\hline$\%$ passing \# 200 & $\mathrm{x}$ & & 8.7 \\
\hline$\%$ passing \#40 & $\mathrm{x}$ & & 20 \\
\hline$\%$ passing \#4 & $\mathrm{x}$ & & 44.7 \\
\hline $\mathrm{D} 10(\mathrm{~mm})$ & $\mathrm{x}$ & & 0.1035 \\
\hline D20 & $\mathrm{x}$ & & 0.425 \\
\hline D30 & $\mathrm{x}$ & & 1.306 \\
\hline D60 & $\mathrm{x}$ & & 10.82 \\
\hline D90 & $\mathrm{x}$ & & 46.19 \\
\hline \multicolumn{3}{|l|}{ User overridable } & index properties \\
\hline Maximum dry unit weight (PCF) & & $\mathrm{x}$ & 127.2 \\
\hline Specific gravity & & $\mathrm{x}$ & 2.7 \\
\hline Sat hydraulic conductivity (ft/hr) & & $\mathrm{x}$ & 0.051 \\
\hline Optimum gravimetric water content $(\%)$ & & $\mathrm{x}$ & 7.4 \\
\hline Degree of saturation of optimum \% & & $\mathrm{x}$ & 61.2 \\
\hline \multicolumn{4}{|c|}{ Overridable soil water characteristic curve: } \\
\hline Af & $\mathrm{x}$ & & 7.255 \\
\hline $\mathrm{Bf}$ & $\mathrm{x}$ & & 1.333 \\
\hline $\mathrm{Cf}$ & $\mathrm{x}$ & & 0.8242 \\
\hline Hf & $\mathrm{x}$ & & 117.4 \\
\hline
\end{tabular}

\section{Layer 4 (Subgrade)}

\begin{tabular}{|c|c|c|c|}
\hline Unbound material (classification): & $\mathrm{x}$ & & $\mathrm{MH}$ \\
\hline Poisson's ratio: 0.35 & & $\mathrm{x}$ & 0.35 \\
\hline Coefficient of lateral pressure $K_{0}: 0.5$ & & $\mathrm{x}$ & 0.5 \\
\hline Modulus (psi) & & $\mathrm{x}$ & 15000 \\
\hline \multicolumn{4}{|c|}{ ICM (Climate model input for this layer's material) } \\
\hline Gradation & $\mathrm{x}$ & & \\
\hline Plasticity index (PI) & $\mathrm{x}$ & & 5 \\
\hline Liquid limit (LL) & $\mathrm{x}$ & & 45 \\
\hline Compact layer & $\mathrm{x}$ & & yes \\
\hline \multicolumn{4}{|l|}{ Index properties from sieve analysis } \\
\hline$\%$ passing \# 200 & $\mathrm{x}$ & & 54.3 \\
\hline$\%$ passing \#40 & $\mathrm{x}$ & & 0 \\
\hline \%passing \#4 & $\mathrm{x}$ & & 86.9 \\
\hline $\mathrm{D} 10(\mathrm{~mm})$ & $\mathrm{x}$ & & 0.0003384 \\
\hline D20 & $\mathrm{x}$ & & 0.001145 \\
\hline D30 & $\mathrm{x}$ & & 0.003876 \\
\hline D60 & $\mathrm{X}$ & & 0.1234 \\
\hline
\end{tabular}




\begin{tabular}{|c|c|c|c|}
\hline & FIX & VARY & Values/Ranges \\
\hline D90 & $\mathrm{x}$ & & 9.109 \\
\hline \multicolumn{3}{|l|}{ User overridable } & index properties \\
\hline Maximum dry unit weight (PCF) & $\mathrm{x}$ & & 119.2 \\
\hline Specific gravity & $\mathrm{x}$ & & 2.7 \\
\hline Sat hydraulic conductivity (ft/hr) & $\mathrm{x}$ & & 9.3 e- 0.007 \\
\hline Optimum gravimetric water content (\%) & $\mathrm{x}$ & & 11.4 \\
\hline Degree of saturation of optimum \% & $\mathrm{x}$ & & 74.4 \\
\hline \multicolumn{4}{|c|}{ Overridable soil water characteristic curve: } \\
\hline Af: 65.23 & $\mathrm{x}$ & & 65.23 \\
\hline Bf: 1.034 & $\mathrm{x}$ & & 1.034 \\
\hline Cf: 0.4994 & $\mathrm{X}$ & & 0.4994 \\
\hline Hf: 500 & $\mathrm{X}$ & & 500 \\
\hline
\end{tabular}

\section{Thermal cracking}

\begin{tabular}{|c|c|c|}
\hline \multirow{3}{*}{$\begin{array}{l}\text { Average tensile strength at } 14 \mathrm{oF}(\mathrm{PSI}): 375.74 \\
\text { Mixture VMA (\%): } 18.6 \\
\text { Agg coefficient of thermal contraction: } 1 \mathrm{e}-006 \\
\text { Mix coefficient of thermal contraction }\end{array}$} & $\mathrm{x}$ & 726.4 \\
\hline & & \\
\hline \multicolumn{3}{|l|}{ Agg coefficient of thermal contraction: $1 \mathrm{e}-006$} \\
\hline & $\mathrm{x}$ & $1.3 \times 10^{\wedge}-05$ \\
\hline
\end{tabular}




\section{MATERIAL SENSITIVITY - STRUCTURE 2 MATERIAL PROPERTIES AND RANGES}

\section{A. Project description}

1 General information FIX VARY Values/Ranges

Design life (year)

Base/subgrade construction month

Pavement construction month

Traffic open

Type design: flexible pavement

\begin{tabular}{|c|c|c|}
\hline $\mathrm{X}$ & & 20 \\
\hline $\mathrm{X}$ & & May \\
\hline $\mathrm{X}$ & & June \\
\hline $\mathrm{X}$ & & July \\
\hline $\mathrm{X}$ & & Flexible \\
\hline
\end{tabular}

2 Site project identification

\begin{tabular}{|l|c|c|c|}
\hline Location & $\mathrm{x}$ & & Morgantown \\
\hline Station/milepost format: miles & $\mathrm{x}$ & & 0.00 \\
\hline Station/milepost begin: & $\mathrm{x}$ & & 0.20 \\
\hline Station/milepost end: & $\mathrm{x}$ & & 5.00 \\
\hline Traffic direction & $\mathrm{x}$ & & east bound \\
\hline
\end{tabular}

\section{Analysis parameters}

\begin{tabular}{|c|c|c|}
\hline Initial IRI & $\mathrm{x}$ & \\
\hline Termal IRI (in/mile) & $\mathrm{x}$ & 172 \\
\hline AC surface down cracking - long cracking $(\mathrm{ft} / \mathrm{mi})$ & $\mathrm{x}$ & 1000 \\
\hline AC bottom up cracking - alligator cracking & $\mathrm{x}$ & 100 \\
\hline Chemically stabilized layer fatigue fracture (\%) & $\mathrm{x}$ & 100 \\
\hline Permanent deformation - Total (in) & $\mathrm{x}$ & 0.75 \\
\hline Permanent deformation - AC only (in) & $\mathrm{x}$ & 0.25 \\
\hline
\end{tabular}

\section{B Inputs}

1 Traffic

\begin{tabular}{|l|c|c|c|}
\hline Initial two-day AADTT & $\mathrm{x}$ & & 2000 \\
\hline Two-away annual average daily traffic (AADT) & $\mathrm{x}$ & & 50 \\
\hline Percent of heavy vehicles (class 4 or higher) & $\mathrm{x}$ & & 2 \\
\hline Number of lanes in design direction & $\mathrm{x}$ & & 50 \\
\hline Percent of trucks in design direction & $\mathrm{x}$ & & 95 \\
\hline Percent of trucks in the design line & $\mathrm{x}$ & & 60 \\
\hline Operational speed: 60 mph & $\mathrm{x}$ & & 4 \\
\hline Traffic growth: compound & &
\end{tabular}




\begin{tabular}{|c|c|c|c|}
\hline Traffic adjustment factors & FIX & VARY & Values/Ranges \\
\hline Monthly adjustment (table) & $\mathrm{x}$ & & Default \\
\hline Vehicle class distribution (Use level 3) & $\mathrm{x}$ & & Default \\
\hline Hourly distribution (table) & $\mathrm{x}$ & & Default \\
\hline \multicolumn{4}{|l|}{ Axle load distribution } \\
\hline Axle type & $\mathrm{x}$ & & Default \\
\hline Single axle & $\mathrm{x}$ & & Default \\
\hline Tandem & $\mathrm{x}$ & & Default \\
\hline Tridem & $\mathrm{x}$ & & Default \\
\hline Quad & $\mathrm{x}$ & & Default \\
\hline \multicolumn{4}{|l|}{ General traffic inputs } \\
\hline Mean wheel location (inches from the lane marking) & $\mathrm{x}$ & & 18 \\
\hline Traffic wander standard deviation (in) & $\mathrm{x}$ & & 10 \\
\hline Design lane width $(\mathrm{ft})$ & $\mathrm{x}$ & & 12 \\
\hline Number axles truck & $\mathrm{x}$ & & Default \\
\hline \multicolumn{4}{|l|}{ Axle configuration } \\
\hline Average axle width $(\mathrm{ft})$ & $\mathrm{x}$ & & 8.5 \\
\hline Dual tire spacing (in) & $\mathrm{x}$ & & 12 \\
\hline Tire pressure (psi) & $\mathrm{x}$ & & 120 \\
\hline \multicolumn{4}{|l|}{ Axle spacing (in) } \\
\hline Tandem axle & $\mathrm{x}$ & & 51.6 \\
\hline Tridem axle & $\mathrm{x}$ & & 49.2 \\
\hline Quad axle & $\mathrm{x}$ & & 49.2 \\
\hline Wheelbase (This does not apply for flexible pavement) & & & \\
\hline
\end{tabular}

\section{Climate}

\begin{tabular}{|c|c|c|}
\hline Station Morgantown, WV & $\mathrm{x}$ & Morgantown \\
\hline Latitude: 39.8 & $\mathrm{x}$ & 39.8 \\
\hline Longitude: -79.55 & $\mathrm{x}$ & -79.55 \\
\hline Elevation $(\mathrm{ft})$ & $\mathrm{x}$ & 1245 \\
\hline Depth of water table (ft) & $\mathrm{x}$ & 20 \\
\hline
\end{tabular}

\section{Structure}

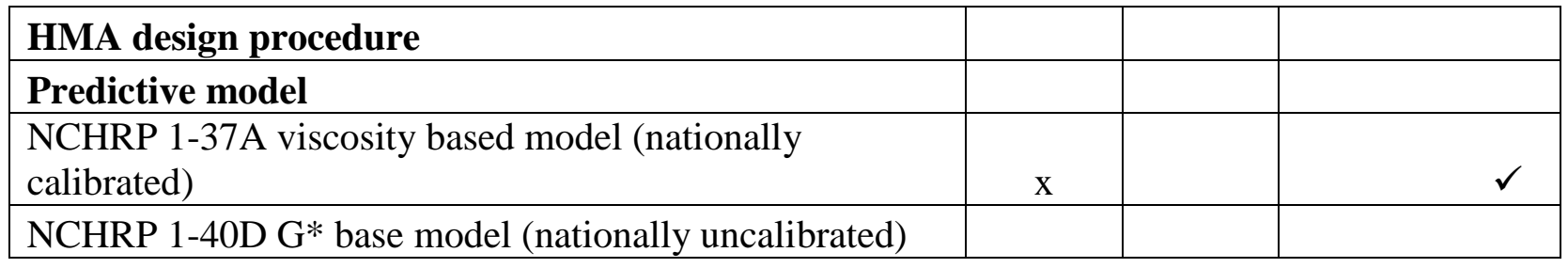


HMA rutting model coefficients

NCHRP 1-37A coefficient

\begin{tabular}{|c|c|c|}
\hline FIX & VARY & Values/Ranges \\
\hline $\mathrm{X}$ & & $\checkmark$ \\
\hline
\end{tabular}

Layer 1 (asphalt concrete layer) $9.5 \mathrm{~mm}$ mix

\begin{tabular}{|c|c|c|c|}
\hline Surface short-wave absorptive & & $\mathrm{x}$ & $0.8-0.90$ \\
\hline Asphalt material type & $\mathrm{x}$ & & $\begin{array}{l}\text { Asphalt } \\
\text { concrete }\end{array}$ \\
\hline Layer thickness (in) & $\mathrm{x}$ & & 2 \\
\hline \multicolumn{4}{|l|}{ Asphalt mix } \\
\hline Cumulative $\%$ retained $\# 3 / 4$ inch sieve & $\mathrm{x}$ & & 0 \\
\hline Cumulative $\%$ retained $\# 3 / 8$ inch sieve & $\mathrm{x}$ & & 7 \\
\hline Cumulative $\%$ retained \#4 inch sieve & $\mathrm{x}$ & & 43 \\
\hline Passing \#200 sieve & $\mathrm{x}$ & & 4.5 \\
\hline \multicolumn{4}{|l|}{ Asphalt binder } \\
\hline Superpave binder grading & $\mathrm{x}$ & & $70-22$ \\
\hline Reference temperature $\left({ }^{\circ} \mathrm{F}\right)$ & $\mathrm{x}$ & $\mathrm{x}$ & 70 \\
\hline \multicolumn{4}{|l|}{ Volumetric properties as bilt } \\
\hline Effective binder content & & $\mathrm{x}$ & $4.5-6.5$ \\
\hline Air voids & & $\mathrm{x}$ & $3-10$ \\
\hline Total unit weight (pcf) & & $\mathrm{x}$ & $145-150$ \\
\hline Poisson's ratio: 0.35 & & $\mathrm{x}$ & $0.25-0.40$ \\
\hline \multicolumn{4}{|l|}{ Thermal properties } \\
\hline Thermal conductivity asphalt (BTU/hr-ft-oF: 0.67 & & $\mathrm{x}$ & $0.5-0.8$ \\
\hline Heat capacity asphalt (BTU/lb-oF): 0.23 & & $\mathrm{x}$ & $0.22-0.5$ \\
\hline
\end{tabular}

Layer 2 (Unbound Base)

\begin{tabular}{|l|c|c|c|}
\hline Unbound material (classification): Crashed Stone & $\mathrm{x}$ & & Crashed Stone \\
\hline Poisson's ratio: 0.35 & & $\mathrm{x}$ & $0.15-0.25$ \\
\hline Coefficient of lateral pressure $\mathrm{K}_{\mathrm{o}}: 0.5$ & & $\mathrm{x}$ & $0.5-0.60$ \\
\hline Modulus (psi) & & $\mathrm{x}$ & $25000-35000$ \\
\hline ICM (Climate model input for this layer's material) & $\mathrm{x}$ & & \\
\hline Gradation & $\mathrm{x}$ & & 5 \\
\hline Plasticity index (PI) & $\mathrm{x}$ & & 45 \\
\hline Liquid limit (LL) & $\mathrm{x}$ & & yes \\
\hline Compact layer & &
\end{tabular}




\begin{tabular}{|c|c|c|c|}
\hline Index properties from sieve analysis & FIX & VARY & Values/Ranges \\
\hline$\%$ passing \# 200 & $\mathrm{x}$ & & 8.7 \\
\hline \%passing \#40 & $\mathrm{x}$ & & 20 \\
\hline \%passing \#4 & $\mathrm{x}$ & & 44.7 \\
\hline $\mathrm{D} 10(\mathrm{~mm})$ & $\mathrm{x}$ & & 0.1035 \\
\hline D20 & $\mathrm{x}$ & & 0.425 \\
\hline D30 & $\mathrm{x}$ & & 1.306 \\
\hline D60 & $\mathrm{x}$ & & 10.82 \\
\hline D90 & $\mathrm{x}$ & & 46.19 \\
\hline \multicolumn{3}{|l|}{ User overridable } & index properties \\
\hline Maximum dry unit weight (PCF) & $\mathrm{x}$ & & 127.2 \\
\hline Specific gravity & $\mathrm{x}$ & & 2.7 \\
\hline Sat hydraulic conductivity (ft/hr) & $\mathrm{x}$ & & 0.051 \\
\hline Optimum gravimetric water content $(\%)$ & $\mathrm{x}$ & & 7.4 \\
\hline Degree of saturation of optimum \% & $\mathrm{x}$ & & 61.2 \\
\hline \multicolumn{4}{|c|}{ Overridable soil water characteristic curve: } \\
\hline Af: 65.23 & $\mathrm{x}$ & & 7.255 \\
\hline Bf: 1.034 & $\mathrm{x}$ & & 1.333 \\
\hline Cf: 0.4994 & $\mathrm{x}$ & & 0.8242 \\
\hline Hf: 500 & $\mathrm{x}$ & & 117.4 \\
\hline
\end{tabular}

Layer 3 (unbound Sub-Base)

\begin{tabular}{|l|c|c|c|}
\hline Unbound material (classification) & $\mathrm{x}$ & & Crushed Gavel \\
\hline Poisson's ratio: 0.35 & & $\mathrm{x}$ & $0.15-0.25$ \\
\hline Coefficient of lateral pressure $\mathrm{K}_{\mathrm{O}}: 0.5$ & & $\mathrm{x}$ & $0.5-0.6$ \\
\hline Modulus (psi) & & $\mathrm{x}$ & $15000-25000$ \\
\hline ICM (Climate model input for this layer's material) & $\mathrm{x}$ & & \\
\hline Gradation & $\mathrm{x}$ & & \multicolumn{2}{|c|}{5} \\
\hline Plasticity index (PI) & $\mathrm{x}$ & & 45 \\
\hline Liquid limit (LL) & $\mathrm{x}$ & & yes \\
\hline Compact layer & & & \multicolumn{2}{|l|}{} \\
\hline
\end{tabular}




\begin{tabular}{|c|c|c|c|}
\hline Index properties from sieve analysis & FIX & VARY & Values/Ranges \\
\hline$\%$ passing \# 200 & $\mathrm{x}$ & & 8.7 \\
\hline$\%$ passing \#40 & $\mathrm{x}$ & & 20 \\
\hline$\%$ passing \#4 & $\mathrm{x}$ & & 44.7 \\
\hline $\mathrm{D} 10(\mathrm{~mm})$ & $\mathrm{x}$ & & 0.1035 \\
\hline D20 & $\mathrm{x}$ & & 0.425 \\
\hline D30 & $\mathrm{x}$ & & 1.306 \\
\hline D60 & $\mathrm{x}$ & & 10.82 \\
\hline D90 & $\mathrm{x}$ & & 46.19 \\
\hline \multicolumn{3}{|l|}{ User overridable } & $\begin{array}{l}\text { index } \\
\text { properties }\end{array}$ \\
\hline Maximum dry unit weight (pcf) & & $\mathrm{x}$ & 127.2 \\
\hline Specific gravity & & $\mathrm{x}$ & 2.7 \\
\hline Sat hydraulic conductivity (ft/hr) & & $\mathrm{x}$ & 0.051 \\
\hline Optimum gravimetric water content (\%) & & $\mathrm{x}$ & 7.4 \\
\hline Degree of saturation of optimum \% & & $\mathrm{x}$ & 61.2 \\
\hline \multicolumn{4}{|c|}{ Overridable soil water characteristic curve: } \\
\hline Af & $\mathrm{x}$ & & 7.255 \\
\hline $\mathrm{Bf}$ & $\mathrm{x}$ & & 1.333 \\
\hline $\mathrm{Cf}$ & $\mathrm{x}$ & & 0.8242 \\
\hline Hf & $\mathrm{x}$ & & 117.4 \\
\hline
\end{tabular}

\section{Layer 4 (Subgrade)}

\begin{tabular}{|c|c|c|c|}
\hline Unbound material (classification): & $\mathrm{x}$ & & $\mathrm{MH}$ \\
\hline Poisson's ratio: 0.35 & & $\mathrm{x}$ & $0.3-0.40$ \\
\hline Coefficient of lateral pressure $\mathrm{K}_{\mathrm{o}}: 0.5$ & & $\mathrm{x}$ & $0.2-0.70$ \\
\hline Modulus (psi) & & $\mathrm{x}$ & $5000-9000$ \\
\hline \multicolumn{4}{|c|}{ ICM (Climate model input for this layer's material) } \\
\hline Gradation & $\mathrm{x}$ & & \\
\hline Plasticity index $(\mathrm{PI})$ & $\mathrm{x}$ & & 5 \\
\hline Liquid limit (LL) & $\mathrm{x}$ & & 45 \\
\hline Compact layer & $\mathrm{x}$ & & yes/not \\
\hline
\end{tabular}




\begin{tabular}{|c|c|c|c|}
\hline Index properties from sieve analysis & FIX & VARY & Values/Ranges \\
\hline \%passing \# 200 & $\mathrm{x}$ & & 54.3 \\
\hline \%passing \#40 & $\mathrm{x}$ & & 0 \\
\hline \%passing \#4 & $\mathrm{x}$ & & 86.9 \\
\hline $\mathrm{D} 10(\mathrm{~mm})$ & $\mathrm{x}$ & & 0.0003384 \\
\hline D20 & $\mathrm{x}$ & & 0.001145 \\
\hline D30 & $\mathrm{x}$ & & 0.003876 \\
\hline D60 & $\mathrm{x}$ & & 0.1234 \\
\hline D90 & $\mathrm{x}$ & & 9.109 \\
\hline \multicolumn{3}{|l|}{ User overridable } & $\begin{array}{l}\text { index } \\
\text { properties }\end{array}$ \\
\hline Maximum dry unit weight (PCF) & $\mathrm{x}$ & & 119.2 \\
\hline Specific gravity & $\mathrm{x}$ & & 2.7 \\
\hline Sat hydraulic conductivity (ft/hr) & $\mathrm{x}$ & & 9.3 e- 0.007 \\
\hline Optimum gravimetric water content $(\%)$ & $\mathrm{x}$ & & 11.4 \\
\hline Degree of saturation of optimum $\%$ & $\mathrm{x}$ & & 74.4 \\
\hline \multicolumn{4}{|c|}{ Overridable soil water characteristic curve: } \\
\hline Af: 65.23 & $\mathrm{x}$ & & 65.23 \\
\hline Bf: 1.034 & $\mathrm{x}$ & & 1.034 \\
\hline Cf: 0.4994 & $\mathrm{x}$ & & 0.4994 \\
\hline Hf: 500 & $\mathrm{x}$ & & 500 \\
\hline
\end{tabular}

\section{Thermal cracking}

\begin{tabular}{|c|c|c|}
\hline Average tensile strength at $140 \mathrm{oF}$ (PSI): 375.74 & $\mathrm{X}$ & $500-1500$ \\
\hline Mixture VMA (\%): 18.6 & & NA \\
\hline Agg coefficient of thermal contraction: $1 \mathrm{e}-006$ & & NA \\
\hline Mix coefficient of thermal contraction & $\mathrm{x}$ & $\begin{array}{l}(2.2-3.4) \times 10^{\wedge} \\
05\end{array}$ \\
\hline
\end{tabular}


Appendix B

LATIN HYPERCUBE SAMPLES 
LHS FOR TRAFFIC SENSITIVITY OF STRUCTURE 1

\begin{tabular}{|c|c|c|c|c|c|c|c|c|c|c|}
\hline RUN & $\begin{array}{l}\text { Two-way } \\
\text { AADT }\end{array}$ & $\begin{array}{l}\% \text { Heavy } \\
\text { Vehicles }\end{array}$ & \begin{tabular}{|l} 
\% Truck \\
Design \\
Direction
\end{tabular} & \begin{tabular}{|l|}
$\%$ Truck \\
Design lane
\end{tabular} & $\begin{array}{l}\text { Operational } \\
\text { Speed }\end{array}$ & $\begin{array}{l}\text { Tracffic } \\
\text { Growth }\end{array}$ & $\begin{array}{l}\text { Mean Wheel } \\
\text { location }\end{array}$ & $\begin{array}{l}\text { Traffic } \\
\text { Wander }\end{array}$ & $\begin{array}{l}\text { Averege } \\
\text { axle width }\end{array}$ & $\begin{array}{l}\text { Dual tire } \\
\text { spacing }\end{array}$ \\
\hline 1 & 34545 & 40.0 & 55.6 & 90.7 & \begin{tabular}{|l|}
57.9 \\
\end{tabular} & 6.1 & $\begin{array}{r}19.7 \\
\end{array}$ & 9.1 & 8.6 & 11.5 \\
\hline 2 & 21515 & 57.6 & 49.5 & 92.2 & 59.1 & 5.3 & 21.0 & 7.1 & 9.7 & 15.4 \\
\hline 3 & 32727 & 52.3 & 56.8 & 73.0 & 63.9 & 7.5 & 15.3 & 12.0 & 9.1 & 15.6 \\
\hline 4 & 39091 & 43.8 & 49.1 & 74.5 & 52.7 & 6.4 & 25.0 & 9.1 & 9.7 & 11.9 \\
\hline 5 & 20303 & 41.6 & 47.1 & 75.1 & 63.0 & 4.3 & 10.9 & 9.7 & 9.6 & 6.3 \\
\hline 6 & 22121 & 52.5 & 59.6 & 83.9 & 45.5 & 5.2 & 7.8 & 10.5 & 8.1 & 8.5 \\
\hline 7 & 34848 & 50.9 & 56.2 & 72.0 & 47.6 & 4.0 & 14.4 & 10.1 & 8.1 & 24.0 \\
\hline 8 & 30303 & 46.7 & 57.6 & 76.8 & 53.0 & 6.8 & 5.6 & 7.5 & 9.1 & 6.2 \\
\hline 9 & 23939 & 59.6 & 41.2 & 93.7 & 55.2 & 6.4 & 23.2 & 9.7 & 8.6 & 8.1 \\
\hline 10 & 35152 & 57.0 & 52.5 & 73.5 & 56.4 & 2.9 & 30.4 & 12.9 & 8.9 & 20.4 \\
\hline 11 & 31515 & 48.7 & 47.5 & 80.1 & 40.3 & 1.8 & 34.1 & 7.4 & 9.0 & 10.8 \\
\hline 12 & 10303 & 49.9 & 49.7 & 73.3 & 57.0 & 7.8 & 18.8 & 11.7 & 8.3 & 11.1 \\
\hline 13 & 38485 & 48.9 & 41.6 & 83.1 & 67.0 & 3.5 & 14.1 & 8.6 & 8.9 & 9.4 \\
\hline 14 & 17879 & 45.5 & 57.4 & 80.6 & 60.9 & 5.2 & 22.5 & 7.6 & 9.3 & 19.4 \\
\hline 15 & 12121 & 47.7 & 59.0 & 88.4 & 54.5 & 2.7 & 36.0 & 9.0 & 8.9 & 7.9 \\
\hline 16 & 33030 & 59.0 & 42.4 & 73.8 & 43.0 & 4.7 & 23.8 & 8.0 & 9.2 & 19.6 \\
\hline 17 & 23030 & 42.8 & 54.5 & 92.7 & 57.6 & 1.3 & 33.5 & 9.2 & 10.0 & 17.5 \\
\hline 18 & 32121 & 41.8 & 58.2 & 85.7 & 65.8 & 5.0 & 26.3 & 12.6 & 9.6 & 15.7 \\
\hline 19 & 11212 & 43.2 & 53.1 & 92.0 & 47.3 & 5.8 & 26.9 & 10.8 & 9.6 & 17.9 \\
\hline 20 & 21212 & 42.2 & 42.6 & 86.9 & 59.7 & 3.6 & 35.7 & 7.8 & 9.2 & 12.5 \\
\hline 21 & 10606 & 53.9 & 52.3 & 81.1 & 46.1 & 4.2 & 20.0 & 12.2 & 8.5 & 20.9 \\
\hline 22 & 18485 & 44.0 & 41.0 & 84.4 & 43.9 & 3.1 & 25.7 & 12.3 & 9.7 & 9.8 \\
\hline 23 & 19091 & 50.7 & 40.8 & 94.2 & 49.7 & 4.1 & 19.1 & 11.6 & 8.7 & 18.6 \\
\hline 24 & 36061 & 52.1 & 47.3 & 91.5 & 41.5 & 1.9 & 27.9 & 11.2 & 9.3 & 19.0 \\
\hline 25 & 33333 & 53.7 & 46.5 & 70.3 & 52.1 & 1.8 & 15.0 & 7.7 & 9.4 & 8.3 \\
\hline 26 & 15758 & 42.0 & 55.8 & 77.8 & 40.9 & 7.2 & 20.3 & 9.4 & 8.4 & 16.3 \\
\hline 27 & 23636 & 57.2 & 58.6 & 91.2 & 48.8 & 5.1 & 12.5 & 11.9 & 9.4 & 16.1 \\
\hline 28 & 13333 & 59.4 & 56.6 & 72.8 & 55.5 & 3.8 & 10.0 & 7.5 & 9.1 & 14.8 \\
\hline 29 & 34242 & 59.2 & 57.2 & 76.6 & 51.8 & 2.8 & 33.8 & 8.9 & 9.9 & 10.6 \\
\hline 30 & 20606 & 49.1 & 55.4 & 76.3 & 45.2 & 2.1 & 35.4 & 12.9 & 9.7 & 14.6 \\
\hline 31 & 13030 & 45.3 & 45.3 & 88.2 & 50.6 & 1.6 & 15.6 & 10.3 & 8.5 & 6.7 \\
\hline 32 & 32424 & 58.2 & 42.8 & 74.0 & 46.4 & 6.3 & 18.2 & 12.5 & 8.6 & 7.7 \\
\hline 33 & 39394 & 58.4 & 50.3 & 87.9 & 47.0 & 4.5 & 7.5 & 9.3 & 8.9 & 21.3 \\
\hline 34 & 26970 & 43.6 & 50.9 & 89.7 & 50.3 & 6.6 & 14.7 & 11.5 & 9.9 & 7.3 \\
\hline 35 & 29394 & 40.8 & 47.7 & 75.8 & 40.6 & 6.7 & 7.2 & 11.0 & 8.8 & 8.6 \\
\hline 36 & 31818 & 47.9 & 45.9 & 71.3 & 64.2 & 1.0 & 32.6 & 9.8 & 9.0 & 13.3 \\
\hline 37 & 36667 & 48.3 & 46.1 & 81.4 & 64.5 & 1.2 & 9.4 & 12.4 & 8.7 & 23.0 \\
\hline 38 & 18182 & 43.0 & 59.4 & 71.5 & 58.2 & 3.9 & 16.3 & 11.8 & 8.3 & 17.1 \\
\hline 39 & 20000 & 41.0 & 58.0 & 79.3 & 42.7 & 1.5 & 19.4 & 8.8 & 9.3 & 12.1 \\
\hline 40 & 40000 & 51.3 & 50.1 & 94.0 & 69.1 & 2.1 & 29.4 & 10.6 & 9.3 & 14.4 \\
\hline 41 & 10000 & 52.9 & 58.8 & 78.3 & 60.0 & 1.4 & 17.8 & 10.6 & 9.5 & 6.9 \\
\hline 42 & 22424 & 49.7 & 53.7 & 84.6 & 66.1 & 3.0 & 8.1 & 8.2 & 8.0 & 13.6 \\
\hline 43 & 12727 & 41.2 & 50.5 & 70.0 & 51.5 & 6.7 & 21.9 & 12.2 & 9.9 & 18.8 \\
\hline 44 & 37273 & 50.3 & 51.5 & 91.7 & 56.7 & 7.6 & 11.9 & 12.7 & 8.4 & 12.3 \\
\hline 45 & 28485 & 47.3 & 45.7 & 76.1 & 64.8 & 5.0 & 13.8 & 12.6 & 8.1 & 5.4 \\
\hline 46 & 30909 & 45.1 & 48.3 & 87.2 & 45.8 & 7.4 & 12.2 & 11.3 & 9.2 & 22.3 \\
\hline 47 & 29091 & 56.4 & 43.0 & 71.8 & 62.7 & 6.5 & 10.3 & 8.3 & 8.3 & 13.8 \\
\hline 48 & 27879 & 51.5 & 44.0 & 80.4 & 44.2 & 2.2 & 5.9 & 8.1 & 8.1 & 13.4 \\
\hline 49 & 11818 & 59.8 & 52.1 & 85.9 & 66.7 & 3.7 & 9.1 & 11.5 & 8.7 & 14.2 \\
\hline 50 & 26061 & 55.8 & 49.3 & 78.6 & 68.5 & 3.4 & 21.6 & 10.2 & 10.0 & 21.9 \\
\hline 51 & 16667 & 55.2 & 46.3 & 83.6 & 67.3 & 7.9 & 18.5 & 11.7 & 9.8 & 10.9 \\
\hline 52 & 17273 & 54.9 & 56.0 & 74.3 & 42.4 & 3.1 & 31.0 & 8.2 & 9.1 & 22.8 \\
\hline 53 & 30606 & 40.2 & 48.5 & 75.6 & 57.3 & 5.5 & 17.5 & 7.9 & 8.0 & 18.4 \\
\hline 54 & 25455 & 44.2 & 48.9 & 90.2 & 43.3 & 5.7 & 17.2 & 7.0 & 9.9 & 17.3 \\
\hline 55 & 38182 & 58.0 & 57.8 & 85.2 & 61.8 & 4.8 & 16.6 & 8.7 & 8.6 & 7.1 \\
\hline 56 & 37576 & 51.9 & 42.0 & 88.9 & 43.6 & 6.9 & 6.3 & 9.9 & 9.2 & 8.8 \\
\hline 57 & 15152 & 56.0 & 50.7 & 90.5 & 40.0 & 2.4 & 11.6 & 8.5 & 8.9 & 20.0 \\
\hline 58 & 36970 & 52.7 & 47.9 & 93.0 & 49.1 & 6.9 & 30.1 & 7.1 & 8.7 & 22.1 \\
\hline 59 & 26667 & 48.5 & 54.7 & 84.1 & 65.5 & 4.3 & 27.5 & 7.2 & 9.8 & 5.2 \\
\hline 60 & 19697 & 48.1 & 40.6 & 75.3 & 54.8 & 7.9 & 23.5 & 8.5 & 9.5 & 19.8 \\
\hline 61 & 16061 & 51.7 & 44.6 & 80.9 & 67.9 & 3.3 & 34.4 & 11.4 & 8.5 & 22.5 \\
\hline 62 & 10909 & 57.8 & 40.0 & 77.3 & 41.8 & 7.0 & 21.3 & 9.2 & 8.3 & 11.7 \\
\hline 63 & 33636 & 49.5 & 43.6 & 70.5 & 50.0 & 4.4 & 8.4 & 12.1 & 9.4 & 16.9 \\
\hline 64 & 18788 & 40.4 & 44.8 & 85.4 & 66.4 & 2.5 & 24.1 & 12.8 & 9.0 & 13.1 \\
\hline 65 & 24848 & 53.3 & 46.7 & 83.4 & 68.2 & 5.5 & 24.7 & 7.2 & 8.1 & 23.6 \\
\hline 66 & 16970 & 46.1 & 51.7 & 77.6 & 61.5 & 5.6 & 27.2 & 7.4 & 8.3 & 5.6 \\
\hline 67 & 28788 & 50.1 & 58.4 & 81.9 & 50.9 & 7.1 & 31.3 & 10.8 & 8.8 & 5.0 \\
\hline 68 & 37879 & 53.5 & 40.2 & 87.7 & 53.9 & 3.8 & 32.2 & 10.0 & 9.6 & 6.0 \\
\hline 69 & 13939 & 55.4 & 43.4 & 82.4 & 70.0 & 2.3 & 9.7 & 7.8 & 9.4 & 11.3 \\
\hline 70 & 24545 & 45.7 & 43.8 & 95.0 & 67.6 & 7.3 & 16.9 & 9.4 & 9.3 & 16.7 \\
\hline 71 & 14848 & 50.5 & 44.4 & 78.8 & 69.4 & 5.7 & 6.9 & 10.4 & 8.7 & 22.7 \\
\hline 72 & 15455 & 49.3 & 48.7 & 82.1 & 53.3 & 4.6 & 5.0 & 10.2 & 10.0 & 18.2 \\
\hline 73 & 35455 & 56.6 & 49.9 & 87.4 & 53.6 & 7.2 & 28.8 & 11.4 & 9.4 & 18.1 \\
\hline 74 & 22727 & 56.2 & 40.4 & 81.6 & 62.4 & 1.1 & 22.2 & 12.5 & 9.8 & 10.4 \\
\hline
\end{tabular}




\begin{tabular}{|c|c|c|c|c|c|c|c|c|c|c|}
\hline RUN & $\begin{array}{l}\text { Two-way } \\
\text { AADT }\end{array}$ & $\begin{array}{l}\% \text { Heavy } \\
\text { Vehicles }\end{array}$ & $\begin{array}{l}\text { \% Truck } \\
\text { Design } \\
\text { Direction }\end{array}$ & $\begin{array}{l}\text { \% Truck } \\
\text { Design lane }\end{array}$ & $\begin{array}{l}\text { Operational } \\
\text { Speed }\end{array}$ & $\begin{array}{l}\text { Tracffic } \\
\text { Growth }\end{array}$ & $\begin{array}{l}\text { Mean Wheel } \\
\text { location }\end{array}$ & $\begin{array}{l}\text { Traffic } \\
\text { Wander }\end{array}$ & $\begin{array}{l}\text { Averege } \\
\text { axle width }\end{array}$ & $\begin{array}{l}\text { Dual tire } \\
\text { spacing }\end{array}$ \\
\hline 75 & 25758 & 46.9 & 53.9 & 93.5 & 55.8 & 3.2 & 32.9 & 12.8 & 8.2 & 12.7 \\
\hline 76 & 20909 & 42.6 & 51.9 & 84.9 & 61.2 & 7.7 & 26.0 & 11.1 & 8.4 & 23.2 \\
\hline 77 & 35758 & 54.3 & 52.9 & 71.0 & 58.5 & 5.4 & 8.8 & 7.7 & 9.5 & 20.5 \\
\hline 78 & 19394 & 46.5 & 45.1 & 88.7 & 46.7 & 8.0 & 11.3 & 8.6 & 8.2 & 9.0 \\
\hline 79 & 39697 & 44.8 & 52.7 & 82.9 & 51.2 & 1.6 & 26.6 & 7.3 & 9.2 & 23.8 \\
\hline 80 & 30000 & 47.5 & 60.0 & 77.1 & 44.8 & 5.9 & 25.4 & 10.7 & 9.5 & 21.7 \\
\hline 81 & 27576 & 43.4 & 53.5 & 89.2 & 47.9 & 2.3 & 10.6 & 10.9 & 8.2 & 21.1 \\
\hline 82 & 21818 & 54.5 & 51.1 & 93.2 & 41.2 & 4.0 & 29.1 & 9.6 & 9.7 & 7.5 \\
\hline 83 & 14545 & 40.6 & 43.2 & 89.4 & 63.3 & 2.6 & 16.0 & 8.9 & 8.6 & 20.7 \\
\hline 84 & 13636 & 56.8 & 46.9 & 72.3 & 49.4 & 4.7 & 20.7 & 10.9 & 9.6 & 6.5 \\
\hline 85 & 17576 & 45.9 & 41.8 & 72.5 & 42.1 & 2.8 & 24.4 & 8.8 & 8.5 & 20.2 \\
\hline 86 & 29697 & 44.6 & 56.4 & 79.1 & 63.6 & 1.7 & 5.3 & 8.3 & 9.8 & 15.0 \\
\hline 87 & 25152 & 41.4 & 45.5 & 74.8 & 48.2 & 4.9 & 30.7 & 11.8 & 8.4 & 10.0 \\
\hline 88 & 28182 & 54.7 & 44.2 & 94.5 & 54.2 & 1.4 & 29.7 & 8.0 & 8.5 & 14.0 \\
\hline 89 & 27273 & 44.4 & 42.2 & 86.4 & 60.3 & 6.2 & 35.1 & 12.0 & 9.8 & 17.7 \\
\hline 90 & 14242 & 60.0 & 54.3 & 79.8 & 44.5 & 7.4 & 31.9 & 10.0 & 9.5 & 15.2 \\
\hline 91 & 16364 & 55.6 & 54.1 & 91.0 & 56.1 & 7.6 & 13.5 & 9.5 & 8.8 & 23.4 \\
\hline 92 & 31212 & 58.8 & 48.1 & 82.6 & 69.7 & 6.0 & 33.2 & $\begin{array}{l}11.1 \\
\end{array}$ & 8.2 & 9.2 \\
\hline 93 & 36364 & 53.1 & 54.9 & 70.8 & 60.6 & 5.9 & 31.6 & 8.4 & 8.4 & 16.5 \\
\hline 94 & 26364 & 54.1 & 59.8 & 86.7 & 58.8 & 1.1 & 22.8 & 9.5 & 8.8 & 21.5 \\
\hline 95 & 12424 & 57.4 & 57.0 & 92.5 & 65.2 & 3.3 & 34.7 & 11.2 & 9.9 & 15.9 \\
\hline 96 & 11515 & 42.4 & 55.2 & 89.9 & 59.4 & 6.2 & 6.6 & 10.3 & 8.8 & 12.9 \\
\hline 97 & 24242 & 51.1 & 51.3 & 94.7 & 68.8 & 4.5 & 12.8 & 12.3 & 9.0 & 5.8 \\
\hline 98 & 23333 & 58.6 & 53.3 & 78.1 & 52.4 & 2.0 & 28.2 & 9.8 & 8.2 & 9.6 \\
\hline 99 & 33939 & 47.1 & 59.2 & 79.6 & 48.5 & 2.6 & 13.1 & 13.0 & 9.1 & 10.2 \\
\hline 100 & 38788 & 46.3 & 41.4 & 86.2 & 62.1 & 3.5 & 28.5 & 10.5 & 8.0 & 19.2 \\
\hline
\end{tabular}


LSH FOR TRAFFIC SENSITIVITY OF STRUCTURE 2

\begin{tabular}{|c|c|c|c|c|c|c|c|c|c|c|}
\hline RUN & $\begin{array}{c}\text { Two-way } \\
\text { AADT }\end{array}$ & $\begin{array}{l}\% \text { Heavy } \\
\text { Vehicles }\end{array}$ & $\begin{array}{l}\% \text { Truck } \\
\text { Design } \\
\text { Direction }\end{array}$ & $\begin{array}{c}\% \text { Truck } \\
\text { Design lane }\end{array}$ & $\begin{array}{l}\text { Operational } \\
\text { Speed }\end{array}$ & $\begin{array}{l}\text { Tracffic } \\
\text { Growth }\end{array}$ & $\begin{array}{l}\text { Mean Wheel } \\
\text { location }\end{array}$ & $\begin{array}{c}\text { Traffic } \\
\text { Wander }\end{array}$ & $\begin{array}{l}\text { Averege } \\
\text { axle width }\end{array}$ & $\begin{array}{l}\text { Dual tire } \\
\text { spacing }\end{array}$ \\
\hline 1 & 3455 & 40.0 & 55.6 & 90.7 & 57.9 & 6.1 & 19.7 & 9.1 & 8.6 & 11.5 \\
\hline 2 & 2152 & 57.6 & 49.5 & 92.2 & 59.1 & 5.3 & 21.0 & 7.1 & 9.7 & 15.4 \\
\hline 3 & 3273 & 52.3 & 56.8 & 73.0 & 63.9 & 7.5 & 15.3 & 12.0 & 9.1 & 15.6 \\
\hline 4 & 3909 & 43.8 & 49.1 & 74.5 & 52.7 & 6.4 & 25.0 & 9.1 & 9.7 & 11.9 \\
\hline 5 & 2030 & 41.6 & 47.1 & 75.1 & 63.0 & 4.3 & 10.9 & 9.7 & 9.6 & 6.3 \\
\hline 6 & 2212 & 52.5 & 59.6 & 83.9 & 45.5 & 5.2 & 7.8 & 10.5 & 8.1 & 8.5 \\
\hline 7 & 3485 & 50.9 & 56.2 & 72.0 & 47.6 & 4.0 & 14.4 & 10.1 & 8.1 & 24.0 \\
\hline 8 & 3030 & 46.7 & 57.6 & 76.8 & 53.0 & 6.8 & 5.6 & 7.5 & 9.1 & 6.2 \\
\hline 9 & 2394 & 59.6 & 41.2 & 93.7 & 55.2 & 6.4 & 23.2 & 9.7 & 8.6 & 8.1 \\
\hline 10 & 3515 & 57.0 & 52.5 & 73.5 & 56.4 & 2.9 & 30.4 & 12.9 & 8.9 & 20.4 \\
\hline 11 & 3152 & 48.7 & 47.5 & 80.1 & 40.3 & 1.8 & 34.1 & 7.4 & 9.0 & 10.8 \\
\hline 12 & 1030 & 49.9 & 49.7 & 73.3 & 57.0 & 7.8 & 18.8 & 11.7 & 8.3 & 11.1 \\
\hline 13 & 3848 & 48.9 & 41.6 & 83.1 & 67.0 & 3.5 & 14.1 & 8.6 & 8.9 & 9.4 \\
\hline 14 & 1788 & 45.5 & 57.4 & 80.6 & 60.9 & 5.2 & 22.5 & 7.6 & 9.3 & 19.4 \\
\hline 15 & 1212 & 47.7 & 59.0 & 88.4 & 54.5 & 2.7 & 36.0 & 9.0 & 8.9 & 7.9 \\
\hline 16 & 3303 & 59.0 & 42.4 & 73.8 & 43.0 & 4.7 & 23.8 & 8.0 & 9.2 & 19.6 \\
\hline 17 & 2303 & 42.8 & 54.5 & 92.7 & 57.6 & 1.3 & 33.5 & 9.2 & 10.0 & 17.5 \\
\hline 18 & 3212 & 41.8 & 58.2 & 85.7 & 65.8 & 5.0 & 26.3 & 12.6 & 9.6 & 15.7 \\
\hline 19 & 1121 & 43.2 & 53.1 & 92.0 & 47.3 & 5.8 & 26.9 & 10.8 & 9.6 & 17.9 \\
\hline 20 & 2121 & 42.2 & 42.6 & 86.9 & 59.7 & 3.6 & 35.7 & 7.8 & 9.2 & 12.5 \\
\hline 21 & 1061 & 53.9 & 52.3 & 81.1 & 46.1 & 4.2 & 20.0 & 12.2 & 8.5 & 20.9 \\
\hline 22 & 1848 & 44.0 & 41.0 & 84.4 & 43.9 & 3.1 & 25.7 & 12.3 & 9.7 & 9.8 \\
\hline 23 & 1909 & 50.7 & 40.8 & 94.2 & 49.7 & 4.1 & 19.1 & 11.6 & 8.7 & 18.6 \\
\hline 24 & 3606 & 52.1 & 47.3 & 91.5 & 41.5 & 1.9 & 27.9 & 11.2 & 9.3 & 19.0 \\
\hline 25 & 3333 & 53.7 & 46.5 & 70.3 & 52.1 & 1.8 & 15.0 & 7.7 & 9.4 & 8.3 \\
\hline 26 & 1576 & 42.0 & 55.8 & 77.8 & 40.9 & 7.2 & 20.3 & 9.4 & 8.4 & 16.3 \\
\hline 27 & 2364 & 57.2 & 58.6 & 91.2 & 48.8 & 5.1 & 12.5 & 11.9 & 9.4 & 16.1 \\
\hline 28 & 1333 & 59.4 & 56.6 & 72.8 & 55.5 & 3.8 & 10.0 & 7.5 & 9.1 & 14.8 \\
\hline 29 & 3424 & 59.2 & 57.2 & 76.6 & 51.8 & 2.8 & 33.8 & 8.9 & 9.9 & 10.6 \\
\hline 30 & 2061 & 49.1 & 55.4 & 76.3 & 45.2 & 2.1 & 35.4 & 12.9 & 9.7 & 14.6 \\
\hline 31 & 1303 & 45.3 & 45.3 & 88.2 & 50.6 & 1.6 & 15.6 & 10.3 & 8.5 & 6.7 \\
\hline 32 & 3242 & 58.2 & 42.8 & 74.0 & 46.4 & 6.3 & 18.2 & 12.5 & 8.6 & 7.7 \\
\hline 33 & 3939 & 58.4 & 50.3 & 87.9 & 47.0 & 4.5 & 7.5 & 9.3 & 8.9 & 21.3 \\
\hline 34 & 2697 & 43.6 & 50.9 & 89.7 & 50.3 & 6.6 & 14.7 & 11.5 & 9.9 & 7.3 \\
\hline 35 & 2939 & 40.8 & 47.7 & 75.8 & 40.6 & 6.7 & 7.2 & 11.0 & 8.8 & 8.6 \\
\hline 36 & 3182 & 47.9 & 45.9 & 71.3 & 64.2 & 1.0 & 32.6 & 9.8 & 9.0 & 13.3 \\
\hline 37 & 3667 & 48.3 & 46.1 & 81.4 & 64.5 & 1.2 & 9.4 & 12.4 & 8.7 & 23.0 \\
\hline 38 & 1818 & 43.0 & 59.4 & 71.5 & 58.2 & 3.9 & 16.3 & 11.8 & 8.3 & 17.1 \\
\hline 39 & 2000 & 41.0 & 58.0 & 79.3 & 42.7 & 1.5 & 19.4 & 8.8 & 9.3 & 12.1 \\
\hline 40 & 4000 & 51.3 & 50.1 & 94.0 & 69.1 & 2.1 & 29.4 & 10.6 & 9.3 & 14.4 \\
\hline 41 & 1000 & 52.9 & 58.8 & 78.3 & 60.0 & 1.4 & 17.8 & 10.6 & 9.5 & 6.9 \\
\hline 42 & 2242 & 49.7 & 53.7 & 84.6 & 66.1 & 3.0 & 8.1 & 8.2 & 8.0 & 13.6 \\
\hline 43 & 1273 & 41.2 & 50.5 & 70.0 & 51.5 & 6.7 & 21.9 & 12.2 & 9.9 & 18.8 \\
\hline 44 & 3727 & 50.3 & 51.5 & 91.7 & 56.7 & 7.6 & 11.9 & 12.7 & 8.4 & 12.3 \\
\hline 45 & 2848 & 47.3 & 45.7 & 76.1 & 64.8 & 5.0 & 13.8 & 12.6 & 8.1 & 5.4 \\
\hline 46 & 3091 & 45.1 & 48.3 & 87.2 & 45.8 & 7.4 & 12.2 & 11.3 & 9.2 & 22.3 \\
\hline 47 & 2909 & 56.4 & 43.0 & 71.8 & 62.7 & 6.5 & 10.3 & 8.3 & 8.3 & 13.8 \\
\hline 48 & 2788 & 51.5 & 44.0 & 80.4 & 44.2 & 2.2 & 5.9 & 8.1 & 8.1 & 13.4 \\
\hline 49 & 1182 & 59.8 & 52.1 & 85.9 & 66.7 & 3.7 & 9.1 & 11.5 & 8.7 & 14.2 \\
\hline 50 & 2606 & 55.8 & 49.3 & 78.6 & 68.5 & 3.4 & 21.6 & 10.2 & 10.0 & 21.9 \\
\hline 51 & 1667 & 55.2 & 46.3 & 83.6 & 67.3 & 7.9 & 18.5 & 11.7 & 9.8 & 10.9 \\
\hline 52 & 1727 & 54.9 & 56.0 & 74.3 & 42.4 & 3.1 & 31.0 & 8.2 & 9.1 & 22.8 \\
\hline 53 & 3061 & 40.2 & 48.5 & 75.6 & 57.3 & 5.5 & 17.5 & 7.9 & 8.0 & 18.4 \\
\hline 54 & 2545 & 44.2 & 48.9 & 90.2 & 43.3 & 5.7 & 17.2 & 7.0 & 9.9 & 17.3 \\
\hline 55 & 3818 & 58.0 & 57.8 & 85.2 & 61.8 & 4.8 & 16.6 & 8.7 & 8.6 & 7.1 \\
\hline 56 & 3758 & 51.9 & 42.0 & 88.9 & 43.6 & 6.9 & 6.3 & 9.9 & 9.2 & 8.8 \\
\hline 57 & 1515 & 56.0 & 50.7 & 90.5 & 40.0 & 2.4 & 11.6 & 8.5 & 8.9 & 20.0 \\
\hline 58 & 3697 & 52.7 & 47.9 & 93.0 & 49.1 & 6.9 & 30.1 & 7.1 & 8.7 & 22.1 \\
\hline 59 & 2667 & 48.5 & 54.7 & 84.1 & 65.5 & 4.3 & 27.5 & 7.2 & 9.8 & 5.2 \\
\hline 60 & 1970 & 48.1 & 40.6 & 75.3 & 54.8 & 7.9 & 23.5 & 8.5 & 9.5 & 19.8 \\
\hline 61 & 1606 & 51.7 & 44.6 & 80.9 & 67.9 & 3.3 & 34.4 & 11.4 & 8.5 & 22.5 \\
\hline 62 & 1091 & 57.8 & 40.0 & 77.3 & 41.8 & 7.0 & 21.3 & 9.2 & 8.3 & 11.7 \\
\hline 63 & 3364 & 49.5 & 43.6 & 70.5 & 50.0 & 4.4 & 8.4 & 12.1 & 9.4 & 16.9 \\
\hline 64 & 1879 & 40.4 & 44.8 & 85.4 & 66.4 & 2.5 & 24.1 & 12.8 & 9.0 & 13.1 \\
\hline 65 & 2485 & 53.3 & 46.7 & 83.4 & 68.2 & 5.5 & 24.7 & 7.2 & 8.1 & 23.6 \\
\hline 66 & 1697 & 46.1 & 51.7 & 77.6 & 61.5 & 5.6 & 27.2 & 7.4 & 8.3 & 5.6 \\
\hline 67 & 2879 & 50.1 & 58.4 & 81.9 & 50.9 & 7.1 & 31.3 & 10.8 & 8.8 & 5.0 \\
\hline 68 & 3788 & 53.5 & 40.2 & 87.7 & 53.9 & 3.8 & 32.2 & 10.0 & 9.6 & 6.0 \\
\hline 69 & 1394 & 55.4 & 43.4 & 82.4 & 70.0 & 2.3 & 9.7 & 7.8 & 9.4 & 11.3 \\
\hline 70 & 2455 & 45.7 & 43.8 & 95.0 & 67.6 & 7.3 & 16.9 & 9.4 & 9.3 & 16.7 \\
\hline 71 & 1485 & 50.5 & 44.4 & 78.8 & 69.4 & 5.7 & 6.9 & 10.4 & 8.7 & 22.7 \\
\hline 72 & 1545 & 49.3 & 48.7 & 82.1 & 53.3 & 4.6 & 5.0 & 10.2 & 10.0 & 18.2 \\
\hline 73 & 3545 & 56.6 & 49.9 & 87.4 & 53.6 & 7.2 & 28.8 & 11.4 & 9.4 & 18.1 \\
\hline 74 & 2273 & 56.2 & 40.4 & 81.6 & 62.4 & 1.1 & 22.2 & 12.5 & 9.8 & 10.4 \\
\hline
\end{tabular}




\begin{tabular}{|c|c|c|c|c|c|c|c|c|c|c|}
\hline RUN & $\begin{array}{c}\text { Two-way } \\
\text { AADT }\end{array}$ & $\begin{array}{l}\% \text { Heavy } \\
\text { Vehicles }\end{array}$ & $\begin{array}{l}\text { \% Truck } \\
\text { Design } \\
\text { Direction }\end{array}$ & $\begin{array}{c}\% \text { Truck } \\
\text { Design lane }\end{array}$ & $\begin{array}{l}\text { Operational } \\
\text { Speed }\end{array}$ & $\begin{array}{l}\text { Tracffic } \\
\text { Growth }\end{array}$ & $\begin{array}{c}\text { Mean Wheel } \\
\text { location }\end{array}$ & $\begin{array}{c}\text { Traffic } \\
\text { Wander }\end{array}$ & $\begin{array}{l}\text { Averege } \\
\text { axle width }\end{array}$ & $\begin{array}{l}\text { Dual tire } \\
\text { spacing }\end{array}$ \\
\hline 75 & 2576 & 46.9 & 53.9 & 93.5 & 55.8 & 3.2 & 32.9 & 12.8 & 8.2 & 12.7 \\
\hline 76 & 2091 & 42.6 & 51.9 & 84.9 & 61.2 & 7.7 & 26.0 & 11.1 & 8.4 & 23.2 \\
\hline 77 & 3576 & 54.3 & 52.9 & 71.0 & 58.5 & 5.4 & 8.8 & 7.7 & 9.5 & 20.5 \\
\hline 78 & 1939 & 46.5 & 45.1 & 88.7 & 46.7 & 8.0 & 11.3 & 8.6 & 8.2 & 9.0 \\
\hline 79 & 3970 & 44.8 & 52.7 & 82.9 & 51.2 & 1.6 & 26.6 & 7.3 & 9.2 & 23.8 \\
\hline 80 & 3000 & 47.5 & 60.0 & 77.1 & 44.8 & 5.9 & 25.4 & 10.7 & 9.5 & 21.7 \\
\hline 81 & 2758 & 43.4 & 53.5 & 89.2 & 47.9 & 2.3 & 10.6 & 10.9 & 8.2 & 21.1 \\
\hline 82 & 2182 & 54.5 & 51.1 & 93.2 & 41.2 & 4.0 & 29.1 & 9.6 & 9.7 & 7.5 \\
\hline 83 & 1455 & 40.6 & 43.2 & 89.4 & 63.3 & 2.6 & 16.0 & 8.9 & 8.6 & 20.7 \\
\hline 84 & 1364 & 56.8 & 46.9 & 72.3 & 49.4 & 4.7 & 20.7 & 10.9 & 9.6 & 6.5 \\
\hline 85 & 1758 & 45.9 & 41.8 & 72.5 & 42.1 & 2.8 & 24.4 & 8.8 & 8.5 & 20.2 \\
\hline 86 & 2970 & 44.6 & 56.4 & 79.1 & 63.6 & 1.7 & 5.3 & 8.3 & 9.8 & 15.0 \\
\hline 87 & 2515 & 41.4 & 45.5 & 74.8 & 48.2 & 4.9 & 30.7 & 11.8 & 8.4 & 10.0 \\
\hline 88 & 2818 & 54.7 & 44.2 & 94.5 & 54.2 & 1.4 & 29.7 & 8.0 & 8.5 & 14.0 \\
\hline 89 & 2727 & 44.4 & 42.2 & 86.4 & 60.3 & 6.2 & 35.1 & 12.0 & 9.8 & 17.7 \\
\hline 90 & 1424 & 60.0 & 54.3 & 79.8 & 44.5 & 7.4 & 31.9 & 10.0 & 9.5 & 15.2 \\
\hline 91 & 1636 & 55.6 & 54.1 & 91.0 & 56.1 & 7.6 & 13.5 & 9.5 & 8.8 & 23.4 \\
\hline 92 & 3121 & 58.8 & 48.1 & 82.6 & 69.7 & 6.0 & 33.2 & 11.1 & 8.2 & 9.2 \\
\hline 93 & 3636 & 53.1 & 54.9 & 70.8 & 60.6 & 5.9 & 31.6 & 8.4 & 8.4 & 16.5 \\
\hline 94 & 2636 & 54.1 & 59.8 & 86.7 & 58.8 & 1.1 & 22.8 & 9.5 & 8.8 & 21.5 \\
\hline 95 & 1242 & 57.4 & 57.0 & 92.5 & 65.2 & 3.3 & 34.7 & 11.2 & 9.9 & 15.9 \\
\hline 96 & 1152 & 42.4 & 55.2 & 89.9 & 59.4 & 6.2 & 6.6 & 10.3 & 8.8 & 12.9 \\
\hline 97 & 2424 & 51.1 & 51.3 & 94.7 & 68.8 & 4.5 & 12.8 & 12.3 & 9.0 & 5.8 \\
\hline 98 & 2333 & 58.6 & 53.3 & 78.1 & 52.4 & 2.0 & 28.2 & 9.8 & 8.2 & 9.6 \\
\hline 99 & 3394 & 47.1 & 59.2 & 79.6 & 48.5 & 2.6 & 13.1 & 13.0 & 9.1 & 10.2 \\
\hline 100 & 3879 & 46.3 & 41.4 & 86.2 & 62.1 & 3.5 & 28.5 & 10.5 & 8.0 & 19.2 \\
\hline
\end{tabular}


Materials Sensitivity for Structure 1

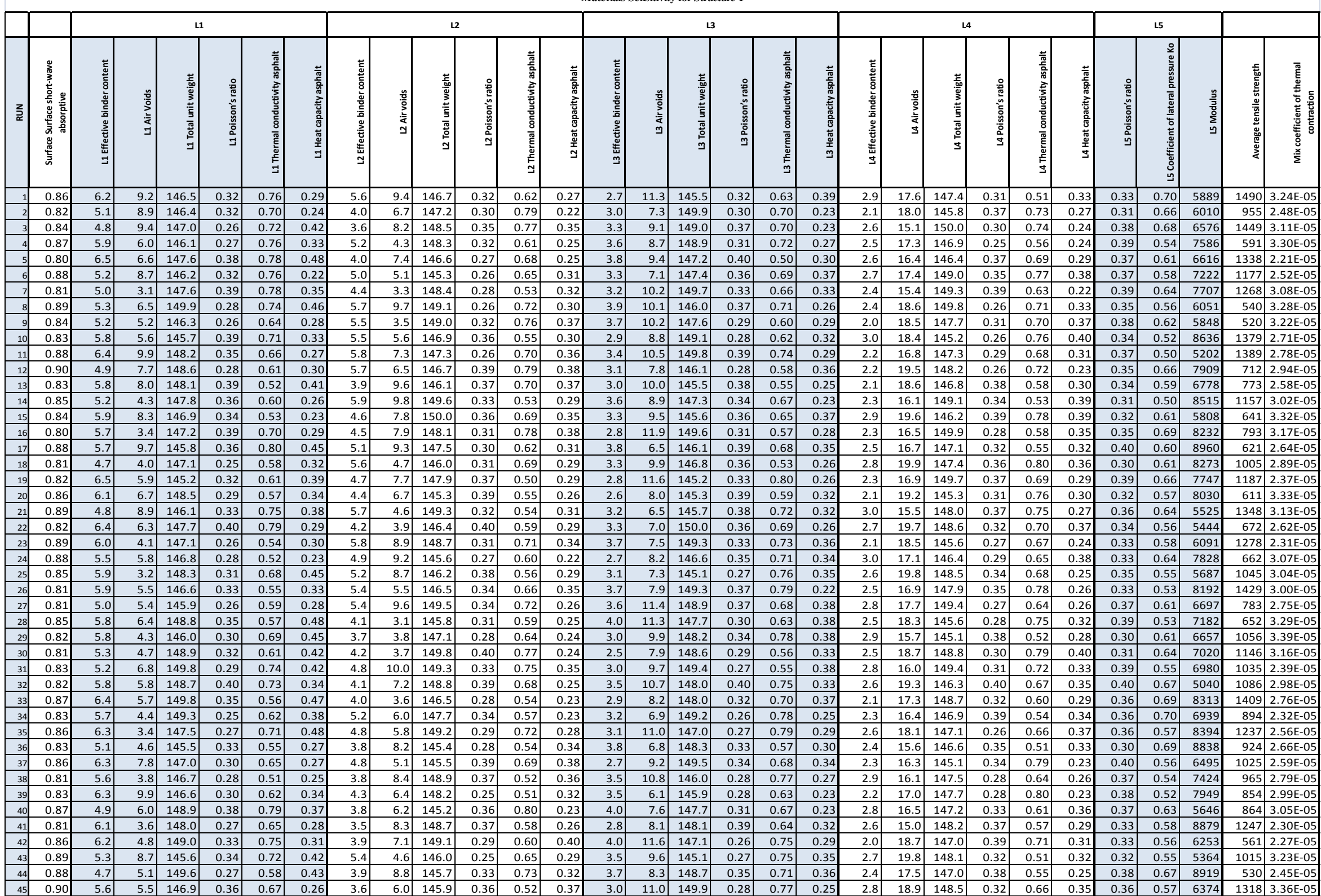




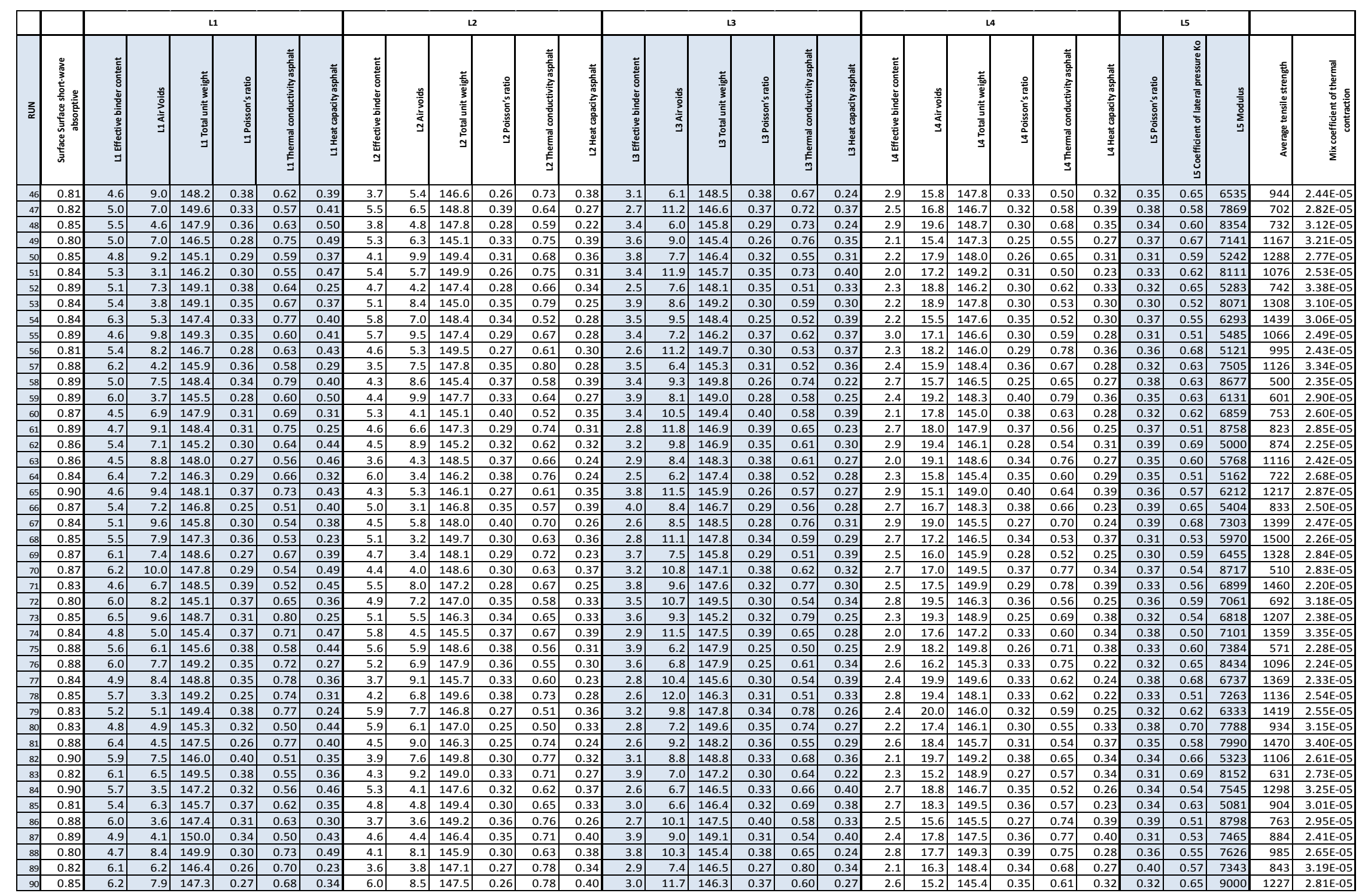




\begin{tabular}{|c|c|c|c|c|c|c|c|c|c|c|c|c|c|c|c|c|c|c|c|c|c|c|c|c|c|c|c|c|c|c|}
\hline & & \multicolumn{6}{|c|}{ L1 } & \multicolumn{6}{|c|}{12} & \multicolumn{6}{|c|}{13} & \multicolumn{6}{|c|}{ L4 } & \multicolumn{3}{|c|}{ L5 } & \multirow{2}{*}{\multicolumn{2}{|c|}{ 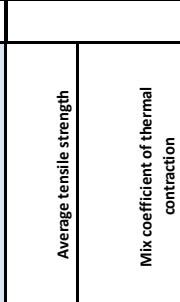 }} \\
\hline za & 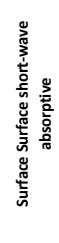 & 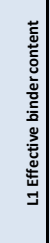 & 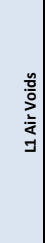 & 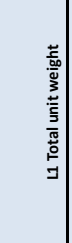 & 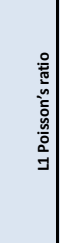 & 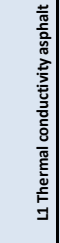 & 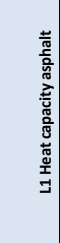 & 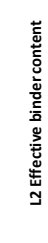 & 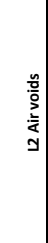 & 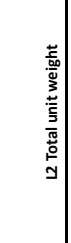 & 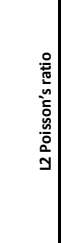 & 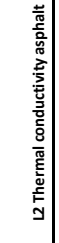 & 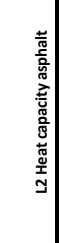 & 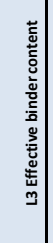 & $\begin{array}{l}\frac{y}{0} \\
\frac{a}{2} \\
\frac{2}{a} \\
m\end{array}$ & 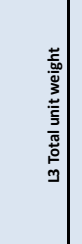 & 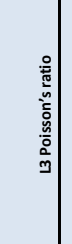 & 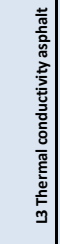 & 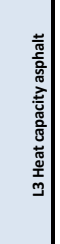 & 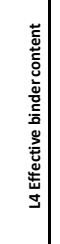 & 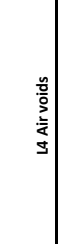 & 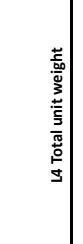 & 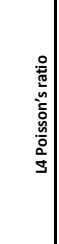 & 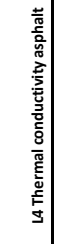 & 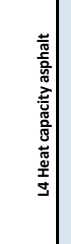 & 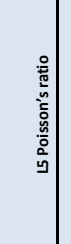 & 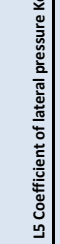 & 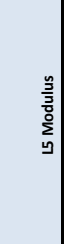 & & \\
\hline 91 & 0.87 & 4.6 & 3.0 & \begin{tabular}{|l|l|}
149.7 \\
\end{tabular} & 0.40 & 0.66 & 0.26 & 5.3 & 5.2 & 148.0 & 0.34 & 0.58 & 0.27 & 2.9 & 6.3 & 146.2 & 0.35 & 0.64 & 0.31 & 2.4 & 16.2 & \begin{tabular}{|l|}
145.9 \\
\end{tabular} & 0.27 & 0.58 & 0.26 & 0.40 & 0.52 & 5929 & 1197 & $2.67 \mathrm{E}-05$ \\
\hline 92 & 0.86 & 4.5 & 4.8 & 148.3 & 0.29 & 0.68 & 0.30 & 4.0 & 4.9 & 146.9 & 0.38 & 0.56 & 0.39 & 3.3 & 8.7 & 145.0 & 0.26 & 0.78 & 0.35 & 2.8 & 15.3 & \begin{tabular}{|l|}
146.8 \\
\end{tabular} & 0.27 & 0.61 & 0.38 & 0.35 & 0.67 & 5566 & 551 & $2.96 \mathrm{E}-05$ \\
\hline 93 & 0.87 & 5.6 & 7.6 & \begin{tabular}{|l|l|}
145.3 \\
\end{tabular} & 0.39 & 0.69 & 0.24 & 5.6 & 7.0 & \begin{tabular}{|c|}
149.7 \\
\end{tabular} & 0.32 & 0.68 & 0.27 & 3.2 & 10.6 & 146.7 & 0.25 & 0.60 & 0.39 & 2.2 & $\begin{array}{ll}18.1 \\
\end{array}$ & \begin{tabular}{|l|}
149.1 \\
\end{tabular} & 0.28 & 0.73 & 0.31 & 0.34 & 0.53 & 8475 & 813 & $2.93 \mathrm{E}-05$ \\
\hline 94 & 0.82 & 5.5 & 5.3 & 147.7 & 0.37 & 0.53 & 0.22 & 4.9 & 9.4 & $\begin{array}{l}147.6 \\
\end{array}$ & 0.38 & 0.75 & 0.33 & 3.7 & \begin{tabular}{|l|}
11.8 \\
\end{tabular} & 148.6 & $\begin{array}{l}0.33 \\
\end{array}$ & 0.56 & 0.24 & 2.8 & 15.9 & \begin{tabular}{|l|l|}
145.8 \\
\end{tabular} & 0.29 & 0.72 & 0.35 & 0.34 & 0.67 & 8596 & 1258 & $2.22 \mathrm{E}-05$ \\
\hline 95 & 0.86 & 5.6 & 3.9 & 145.0 & 0.37 & 0.78 & 0.32 & 5.9 & 7.9 & 145.8 & 0.29 & 0.78 & 0.39 & 2.5 & 10.9 & 147.0 & 0.27 & 0.62 & 0.31 & 2.5 & \begin{tabular}{ll|}
17.9 \\
\end{tabular} & \begin{tabular}{|l|}
145.7 \\
\end{tabular} & 0.33 & 0.74 & 0.29 & 0.36 & 0.64 & 6414 & 803 & $2.70 \mathrm{E}-05$ \\
\hline 96 & 0.87 & 5.1 & 8.6 & 145.4 & 0.34 & 0.68 & 0.47 & 5.0 & 7.5 & \begin{tabular}{|l|l|}
149.9 \\
\end{tabular} & 0.35 & 0.51 & 0.34 & 2.7 & 10.4 & 148.8 & 0.29 & 0.66 & 0.24 & 2.2 & 16.6 & \begin{tabular}{|l|}
148.8 \\
\end{tabular} & 0.39 & 0.59 & 0.36 & 0.31 & 0.66 & 6172 & 682 & $2.92 \mathrm{E}-05$ \\
\hline 97 & 0.85 & 4.9 & 9.3 & 149.4 & 0.31 & 0.59 & 0.38 & 4.2 & 8.7 & 148.3 & 0.31 & 0.55 & 0.33 & 3.3 & 6.4 & 148.7 & 0.36 & 0.72 & 0.35 & 2.6 & 19.0 & \begin{tabular}{|l|}
147.6 \\
\end{tabular} & 0.29 & $\begin{array}{ll}0.73 \\
\end{array}$ & 0.37 & 0.39 & 0.52 & 7667 & 1480 & $3.27 \mathrm{E}-05$ \\
\hline 90 & 0.82 & 4.7 & 8.5 & 149.7 & 0.34 & 0.52 & 0.36 & 4.7 & 3.0 & 145.6 & $\begin{array}{l}0.27 \\
\end{array}$ & 0.53 & 0.23 & 3.7 & 8.5 & 147.3 & 0.34 & 0.75 & 0.28 & 2.2 & 16.6 & \begin{tabular}{|l|}
149.6 \\
\end{tabular} & 0.4 & 0.63 & 0.30 & 0.31 & 0.68 & 5606 & 914 & $2.88 \mathrm{E}-05$ \\
\hline 99 & 0.86 & 5.3 & 8.1 & 149.5 & 0.33 & 0.72 & 0.49 & 5.0 & 5.0 & 148.2 & $\begin{array}{l}0.39 \\
\end{array}$ & 0.57 & 0.30 & 2.7 & 7.8 & 148.4 & 0.32 & 0.52 & 0.31 & 2.1 & 15.3 & \begin{tabular}{|l|}
145.2 \\
\end{tabular} & 0.38 & 0.72 & 0.31 & 0.38 & 0.62 & 5727 & 581 & $2.36 \mathrm{E}-05$ \\
\hline 100 & 0.83 & 6.3 & 9.5 & 149.0 & 0.37 & 0.65 & 0.32 & 4.9 & 6.3 & 148.9 & 0.38 & 0.74 & 0.37 & 3.1 & 6.7 & 146.8 & 0.27 & $\begin{array}{ll}0.53 \\
\end{array}$ & 0.31 & 3.0 & 19.1 & \begin{tabular}{|l|}
149.7 \\
\end{tabular} & 0.36 & 0.62 & 0.35 & 0.34 & 0.59 & 8556 & 975 & $2.72 \mathrm{E}-05$ \\
\hline 101 & 0.89 & 6.5 & 9.3 & 146.9 & 0.32 & 0.72 & 0.36 & 3.8 & 8.3 & \begin{tabular}{|c|}
147.7 \\
\end{tabular} & 0.31 & 0.75 & 0.39 & 2.5 & \begin{tabular}{l|l|}
10.9 \\
\end{tabular} & 146.7 & 0.28 & 0.52 & 0.35 & 2.3 & 15.2 & \begin{tabular}{|l|l}
145.4 \\
\end{tabular} & 0.40 & 0.54 & 0.33 & 0.32 & 0.53 & 6387 & \begin{tabular}{|l|l|}
997 \\
\end{tabular} & $2.77 \mathrm{E}-05$ \\
\hline 102 & 0.82 & 5.5 & 7.1 & 146.5 & 0.27 & 0.57 & 0.41 & 5.6 & 5.7 & $\begin{array}{l}147.0 \\
\end{array}$ & 0.25 & 0.55 & $\begin{array}{l}0.37 \\
\end{array}$ & 3.4 & 10.4 & 145.1 & 0.35 & 0.75 & 0.38 & 2.5 & 20.0 & \begin{tabular}{|l|l|}
149.7 \\
\end{tabular} & 0.31 & 0.61 & 0.24 & 0.39 & 0.56 & 6025 & \begin{tabular}{|l|}
1279 \\
\end{tabular} & $3.07 \mathrm{E}-05$ \\
\hline 103 & 0.87 & 5.4 & 6.0 & $\begin{array}{l}145.1 \\
\end{array}$ & 0.31 & 0.68 & 0.28 & 5.8 & 8.2 & \begin{tabular}{|l|l|}
146.9 \\
\end{tabular} & $\begin{array}{l}0.38 \\
\end{array}$ & 0.53 & 0.27 & 3.2 & $\begin{array}{l}8.7 \\
\end{array}$ & 146.7 & 0.29 & 0.75 & 0.30 & 2.9 & 18.0 & \begin{tabular}{|l|}
145.6 \\
\end{tabular} & 0.31 & 0.53 & 0.39 & 0.39 & 0.57 & 7211 & 857 & $2.98 \mathrm{E}-05$ \\
\hline 104 & 0.87 & 5.8 & 7.6 & $\begin{array}{l}146.4 \\
\end{array}$ & 0.36 & 0.53 & 0.33 & 3.7 & 9.8 & 148.9 & 0.38 & 0.50 & 0.24 & 3.0 & 11.2 & 147.6 & 0.34 & 0.58 & 0.24 & 2.8 & 17.8 & \begin{tabular}{|l|}
148.4 \\
\end{tabular} & 0.25 & 0.64 & 0.24 & 0.35 & 0.56 & 7734 & 616 & $2.51 \mathrm{E}-05$ \\
\hline 105 & 0.83 & 5.1 & 7.1 & 148.2 & 0.36 & 0.55 & 0.29 & 5.8 & 6.4 & 149.2 & 0.28 & 0.51 & 0.35 & 3.2 & 8.2 & 148.2 & 0.39 & \begin{tabular}{ll|}
0.58 \\
\end{tabular} & 0.29 & 2.6 & 16.3 & \begin{tabular}{|l|}
148.8 \\
\end{tabular} & 0.27 & 0.54 & 0.37 & 0.40 & 0.64 & 5482 & 1234 & $2.46 \mathrm{E}-05$ \\
\hline 106 & 0.86 & 6.0 & 7.4 & 149.0 & 0.26 & 0.74 & 0.49 & 5.1 & 7.3 & \begin{tabular}{|l|l|}
147.3 \\
\end{tabular} & 0.31 & 0.59 & 0.32 & 2.7 & 11.9 & 149.8 & 0.34 & 0.68 & 0.23 & 2.7 & 17.6 & \begin{tabular}{|l|l|}
148.4 \\
\end{tabular} & 0.37 & 0.54 & 0.37 & 0.36 & 0.59 & 8518 & 1068 & $3.32 \mathrm{E}-05$ \\
\hline 107 & 0.87 & 5.8 & 3.5 & $\begin{array}{ll}148.1 \\
\end{array}$ & 0.40 & 0.58 & 0.46 & 5.7 & 5.5 & $\begin{array}{l}145.4 \\
1\end{array}$ & \begin{tabular}{|l|}
0.38 \\
\end{tabular} & 0.65 & 0.36 & 3.8 & 7.5 & 146.9 & 0.31 & 0.72 & 0.22 & 2.2 & 16.4 & 149.2 & 0.34 & 0.63 & 0.39 & 0.32 & 0.59 & 5945 & 1440 & $2.54 \mathrm{E}-05$ \\
\hline 108 & 0.85 & 6.0 & 3.7 & \begin{tabular}{|l|}
148.4 \\
\end{tabular} & 0.29 & 0.70 & 0.35 & 5.2 & 4.7 & $\begin{array}{l}148.6 \\
\end{array}$ & 0.33 & 0.75 & 0.22 & 2.9 & 8.9 & \begin{tabular}{|l|}
147.1 \\
\end{tabular} & 0.36 & 0.70 & 0.29 & 2.0 & \begin{tabular}{|l|}
15.3 \\
\end{tabular} & \begin{tabular}{|l|}
146.3 \\
\end{tabular} & 0.26 & 0.65 & 0.23 & 0.38 & 0.55 & 6246 & 791 & $3.04 \mathrm{E}-05$ \\
\hline 109 & 0.85 & 5.8 & 8.5 & 145.3 & 0.29 & 0.67 & 0.28 & 5.4 & 3.9 & 149.2 & 0.29 & 0.58 & 0.35 & 3.6 & 7.6 & 148.9 & 0.30 & 0.57 & 0.27 & 2.7 & 15.3 & \begin{tabular}{|l|l|}
145.9 \\
\end{tabular} & 0.27 & 0.73 & 0.26 & 0.39 & 0.52 & 8739 & 1028 & $2.25 \mathrm{E}-05$ \\
\hline 110 & 0.89 & 5.6 & 3.6 & 147.4 & 0.39 & 0.72 & 0.33 & 3.9 & 3.4 & \begin{tabular}{|l|l|}
146.7 \\
\end{tabular} & 0.39 & 0.59 & 0.27 & 3.4 & 11.2 & 146.0 & 0.32 & 0.61 & 0.24 & 2.6 & 15.9 & \begin{tabular}{|l|}
149.0 \\
\end{tabular} & 0.28 & 0.60 & 0.30 & 0.32 & 0.65 & 8618 & 1369 & $3.14 \mathrm{E}-05$ \\
\hline 111 & 0.80 & 5.1 & 4.4 & 149.3 & 0.25 & 0.54 & 0.37 & 4.0 & 7.2 & 148.5 & 0.28 & 0.61 & 0.28 & 3.7 & 9.1 & 147.3 & 0.39 & 0.69 & 0.29 & 2.8 & \begin{tabular}{|l|l|}
17.7 \\
\end{tabular} & \begin{tabular}{|l|l|}
149.8 \\
\end{tabular} & 0.2 & 0.59 & 0.38 & 0.33 & 0.53 & 6528 & 1158 & $2.30 \mathrm{E}-05$ \\
\hline 112 & 0.82 & 6.2 & 8.6 & 146.2 & 0.28 & 0.64 & 0.44 & 4.6 & 9.8 & 147.9 & 0.26 & 0.76 & 0.37 & 3.3 & 7.0 & 148.0 & 0.28 & 0.67 & 0.28 & 2.8 & 19.9 & \begin{tabular}{|l|}
147.3 \\
\end{tabular} & 0.33 & 0.60 & 0.32 & 0.36 & 0.68 & 6186 & 1495 & $2.22 \mathrm{E}-05$ \\
\hline 113 & 0.84 & 5.7 & 8.9 & 145.0 & 0.32 & 0.70 & 0.27 & 5.0 & 8.6 & \begin{tabular}{|l|l|}
148.1 \\
\end{tabular} & 0.38 & 0.72 & 0.32 & 2.7 & 6.7 & 145.4 & 0.39 & 0.80 & 0.25 & 2.9 & 16.5 & \begin{tabular}{|l|}
145.3 \\
\end{tabular} & 0.30 & 0.78 & 0.35 & 0.34 & 0.52 & 7774 & 1033 & $2.57 \mathrm{E}-05$ \\
\hline 114 & 0.88 & 4.8 & 6.6 & \begin{tabular}{|c|}
149.8 \\
\end{tabular} & 0.28 & 0.61 & 0.48 & 4.1 & 9.1 & \begin{tabular}{|l|l|}
149.3 \\
\end{tabular} & 0.32 & 0.74 & 0.26 & 3.3 & 7.0 & 147.0 & 0.30 & $\begin{array}{ll}0.63 \\
\end{array}$ & 0.37 & 2.7 & 15.3 & \begin{tabular}{|l|l|}
147.9 \\
\end{tabular} & 0.34 & 0.57 & $\begin{array}{l}0.27 \\
\end{array}$ & 0.30 & 0.51 & 8980 & 776 & $2.97 \mathrm{E}-05$ \\
\hline 115 & 0.90 & 5.4 & 9.0 & 147.3 & 0.25 & 0.65 & 0.24 & 4.1 & 9.4 & $\begin{array}{l}145.7 \\
\end{array}$ & 0.40 & 0.65 & 0.32 & 3.6 & \begin{tabular}{l|l|}
11.7 \\
\end{tabular} & 145.5 & 0.27 & 0.74 & 0.25 & 2.3 & \begin{tabular}{|l|}
18.3 \\
\end{tabular} & \begin{tabular}{|l|}
149.3 \\
\end{tabular} & 0.32 & 0.69 & 0.24 & 0.34 & 0.52 & 8638 & 671 & $2.54 \mathrm{E}-05$ \\
\hline 116 & 0.83 & 5.5 & 9.7 & \begin{tabular}{|l|l|}
146.9 \\
\end{tabular} & 0.31 & 0.72 & 0.48 & 5.9 & 4.9 & \begin{tabular}{|l|l|}
145.4 \\
\end{tabular} & 0.31 & 0.79 & 0.30 & 3.6 & 8.2 & 149.0 & 0.27 & 0.65 & 0.3 & 2.3 & $\begin{array}{ll}18.3 \\
\end{array}$ & \begin{tabular}{|l|}
148.3 \\
\end{tabular} & 0.3 & 0.69 & 0.24 & 0.34 & 0.69 & 7874 & 595 & $3.40 \mathrm{E}-05$ \\
\hline 117 & 0.83 & 5.6 & 8.4 & 149.8 & 0.35 & 0.58 & 0.46 & 5.6 & 4.8 & 149.4 & 0.27 & 0.56 & 0.26 & 2.7 & 7.9 & $\begin{array}{l}148.7 \\
\end{array}$ & 0.26 & 0.78 & 0.39 & 2.4 & 15.4 & \begin{tabular}{|l|}
147.1 \\
\end{tabular} & 0.39 & 0.54 & 0.23 & 0.31 & 0.59 & 5985 & 1264 & $2.65 \mathrm{E}-05$ \\
\hline 118 & 0.88 & 5.3 & 3.5 & \begin{tabular}{|l|l|}
145.7 \\
\end{tabular} & 0.35 & 0.68 & 0.26 & 5.1 & 7.5 & \begin{tabular}{|l|l|}
145.1 \\
\end{tabular} & 0.29 & 0.69 & 0.36 & 3.1 & \begin{tabular}{|l|}
11.8 \\
\end{tabular} & 145.6 & 0.29 & 0.51 & 0.23 & 2.5 & 17.2 & \begin{tabular}{|l|}
147.3 \\
\end{tabular} & 0.32 & 0.59 & 0.34 & 0.32 & 0.55 & 5442 & 560 & $3.11 \mathrm{E}-05$ \\
\hline 119 & 0.81 & 5.7 & 8.1 & \begin{tabular}{|l|}
149.4 \\
\end{tabular} & 0.33 & 0.58 & 0.35 & 5.5 & 8.9 & $\begin{array}{l}149.6 \\
\end{array}$ & $\begin{array}{l}0.38 \\
\end{array}$ & 0.74 & 0.37 & 3.8 & 8.8 & 149.9 & 0.26 & 0.7 & 0.3 & 2.4 & 16.0 & \begin{tabular}{|l|l|}
147.8 \\
\end{tabular} & 0.3 & 0.73 & .37 & 0.39 & 0.5 & 6608 & 937 & $2.25 \mathrm{E}-05$ \\
\hline 120 & 0.81 & 5.5 & 9.6 & \begin{tabular}{|l|}
147.0 \\
\end{tabular} & 0.30 & 0.71 & 0.43 & 5.7 & 5.4 & 148.4 & 0.37 & 0.57 & 0.29 & 3.9 & 9.5 & 148.5 & 0.25 & 0.61 & 0.4 & 2.6 & 15.9 & \begin{tabular}{|l|}
145.2 \\
\end{tabular} & 0.2 & 0.55 & 0.26 & 0.34 & 0.50 & 5080 & 1384 & $3.01 \mathrm{E}-05$ \\
\hline 121 & 0.88 & 4.9 & 9.3 & \begin{tabular}{|l|}
148.1 \\
\end{tabular} & 0.25 & 0.76 & 0.45 & 4.6 & 9.6 & $\begin{array}{l}146.9 \\
\end{array}$ & 0.37 & 0.61 & $\begin{array}{l}0.33 \\
\end{array}$ & 3.2 & 7.7 & 145.0 & 0.26 & 0.59 & 0.23 & 2.0 & 15.2 & \begin{tabular}{|l|}
145.9 \\
\end{tabular} & 0.35 & 0.55 & 0.37 & 0.32 & $\begin{array}{l}0.67 \\
\end{array}$ & 7513 & 1078 & $3.13 \mathrm{E}-05$ \\
\hline 122 & 0.81 & 5.6 & 4.6 & 148.3 & 0.39 & 0.79 & 0.29 & 5.9 & 4.7 & 145.1 & 0.39 & 0.62 & 0.35 & 3.3 & 8.4 & 146.8 & 0.38 & 0.77 & 0.32 & 2.1 & 18.7 & \begin{tabular}{|l|l|}
147.9 \\
\end{tabular} & 0.30 & 0.65 & 0.36 & 0.32 & 0.63 & 5844 & 510 & $2.62 \mathrm{E}-05$ \\
\hline 123 & 0.81 & 4.7 & 7.5 & 148.2 & 0.30 & 0.62 & 0.38 & 4.0 & 3.2 & $\begin{array}{l}148.6 \\
\end{array}$ & 0.37 & 0.52 & 0.37 & 2.9 & 7.7 & 146.2 & 0.29 & 0.56 & 0.31 & 2.4 & 19.9 & \begin{tabular}{|l|}
146.0 \\
\end{tabular} & 0.32 & 0.71 & 0.39 & 0.35 & 0.60 & 5563 & 1364 & $3.17 \mathrm{E}-05$ \\
\hline 124 & 0.89 & 6.0 & 5.9 & 149.0 & 0.35 & 0.77 & 0.34 & 5.8 & 5.3 & 145.2 & 0.40 & 0.55 & 0.28 & 2.7 & 10.3 & 148.1 & 0.36 & 0.60 & 0.2 & 2.2 & 18.7 & \begin{tabular}{|l|}
148.8 \\
\end{tabular} & 0.30 & 0.71 & 0.25 & 0.30 & 0.51 & 7995 & 545 & $3.26 \mathrm{E}-05$ \\
\hline 125 & 0.83 & 6.3 & 9.9 & 145.6 & 0.33 & 0.61 & 0.23 & 4.3 & 5.5 & 145.9 & 0.27 & 0.57 & 0.26 & 2.8 & 7.1 & 147.6 & 0.37 & 0.51 & & 2.2 & 17.0 & \begin{tabular}{|l|}
146.3 \\
\end{tabular} & & 0.77 & 0.29 & 0.34 & & 6568 & 555 & $2.44 \mathrm{E}-05$ \\
\hline 126 & 0.81 & 5.9 & 6.8 & 146.3 & 0.30 & 0.59 & 0.25 & 4.7 & 9.5 & 145.5 & 0.35 & 0.58 & 0.30 & 3.5 & 6.9 & 149.6 & 0.36 & 0.74 & 0.31 & 2.3 & 16.4 & \begin{tabular}{|l|}
146.4 \\
\end{tabular} & 0.26 & 0.78 & 0.35 & 0.38 & 0.68 & 6226 & 1148 & $3.29 \mathrm{E}-05$ \\
\hline 127 & 0.81 & 6.0 & 6.8 & 149.0 & 0.32 & 0.74 & 0.27 & 3.5 & 5.2 & \begin{tabular}{|l|l|}
145.3 \\
\end{tabular} & 0.35 & 0.73 & 0.25 & 3.2 & \begin{tabular}{l|l|}
11.9 \\
\end{tabular} & 149.1 & 0.35 & 0.52 & & 2.8 & 15.2 & \begin{tabular}{|l|l|}
148.3 \\
148
\end{tabular} & 0.3 & 0.74 & & 0.39 & & 5181 & 626 & $2.95 \mathrm{E}-05$ \\
\hline 128 & 0.85 & 5.3 & 3.6 & 146.9 & 0.36 & 0.63 & 0.46 & 5.9 & 9.3 & 148.0 & 0.31 & 0.52 & 0.24 & 3.7 & 8.6 & 146.2 & 0.36 & 0.66 & 0.34 & 2.6 & $\begin{array}{ll}19.1 \\
\end{array}$ & \begin{tabular}{|l|}
148.0 \\
\end{tabular} & 0.39 & 0.66 & 0.28 & 0.34 & 0.62 & 5462 & 1430 & $2.74 \mathrm{E}-05$ \\
\hline 129 & 0.86 & 6.3 & 4.9 & 145.2 & 0.27 & 0.67 & 0.24 & 5.9 & 4.7 & 149.1 & 0.31 & 0.63 & $\begin{array}{l}0.37 \\
\end{array}$ & 2.6 & 8.4 & 146.3 & 0.39 & $\begin{array}{l}0.63 \\
\end{array}$ & 0.37 & 2.5 & 18.4 & \begin{tabular}{|l|l|}
145.8 \\
\end{tabular} & 0.33 & 0.74 & 0.26 & 0.35 & 0.55 & 7472 & 1048 & $2.66 \mathrm{E}-05$ \\
\hline 130 & 0.87 & 4.9 & 3.2 & 148.8 & 0.40 & 0.56 & 0.35 & 4.0 & 6.4 & 149.0 & 0.35 & 0.58 & 0.32 & 3.4 & 11.3 & 149.5 & 0.40 & 0.70 & & 2.5 & 17.5 & \begin{tabular}{|l|}
149.1 \\
\end{tabular} & 0.39 & 0.63 & 0.32 & 0.37 & 0.54 & 7392 & 530 & $2.41 \mathrm{E}-05$ \\
\hline 131 & 0.89 & 5.4 & 5.4 & 146.5 & 0.26 & 0.78 & 0.46 & 4.0 & 5.9 & $\begin{array}{l}148.2 \\
\end{array}$ & 0.28 & 0.52 & 0.31 & 2.7 & 11.5 & 145.2 & 0.31 & 0.65 & 0.29 & 2.8 & 18.2 & 146.9 & 0.30 & 0.52 & 0.38 & 0.39 & 0.57 & 6829 & 1304 & $2.32 \mathrm{E}-05$ \\
\hline 132 & 0.81 & 4.9 & 6.0 & $\begin{array}{l}146.6 \\
\end{array}$ & 0.38 & 0.77 & 0.30 & 3.5 & 6.1 & 1469 & & 0.70 & 0.25 & 2.9 & \begin{tabular}{|l|}
7.7 \\
\end{tabular} & 145.2 & 0.28 & 0.73 & & 2.2 & & 1487 & & & & & & 5683 & 1455 & $3.12 \mathrm{E}-05$ \\
\hline 133 & 0.89 & 6.5 & 3.0 & \begin{tabular}{|l|l|}
147.4 \\
\end{tabular} & 0.33 & 0.58 & 0.27 & 4.8 & 5.1 & $\begin{array}{l}146.7 \\
\end{array}$ & 0.31 & 0.80 & 0.35 & 2.8 & \begin{tabular}{|l|}
11.6 \\
\end{tabular} & 147.8 & 0.32 & 0.72 & 0.39 & 2.6 & 19.0 & \begin{tabular}{|l|}
148.0 \\
\end{tabular} & 0.33 & 0.63 & 0.36 & 0.39 & 0.65 & 5643 & 1294 & $3.26 \mathrm{E}-05$ \\
\hline 134 & 0.86 & 5.3 & 4.0 & 148.0 & 0.38 & 0.71 & 0.41 & 4.6 & 4.4 & 150.0 & 0.27 & 0.61 & 0.23 & 2.5 & 10.9 & 145.4 & 0.30 & 0.69 & 0.34 & 2.5 & 17.3 & 146.5 & 0.38 & 0.56 & 0.28 & 0.38 & 0.61 & 5241 & 525 & $2.28 \mathrm{E}-05$ \\
\hline 135 & 0.82 & 6.5 & 8.4 & 148.7 & 0.27 & 0.67 & 0.28 & 5.4 & 6.6 & 147.2 & 0.31 & 0.75 & 0.31 & 3.3 & 10.3 & 146.0 & 0.29 & 0.55 & 0.23 & 2.2 & 19.3 & 147.5 & 0.29 & 0.51 & 0.36 & 0.38 & 0.56 & 879 & 1410 & $3.08 \mathrm{E}-05$ \\
\hline
\end{tabular}




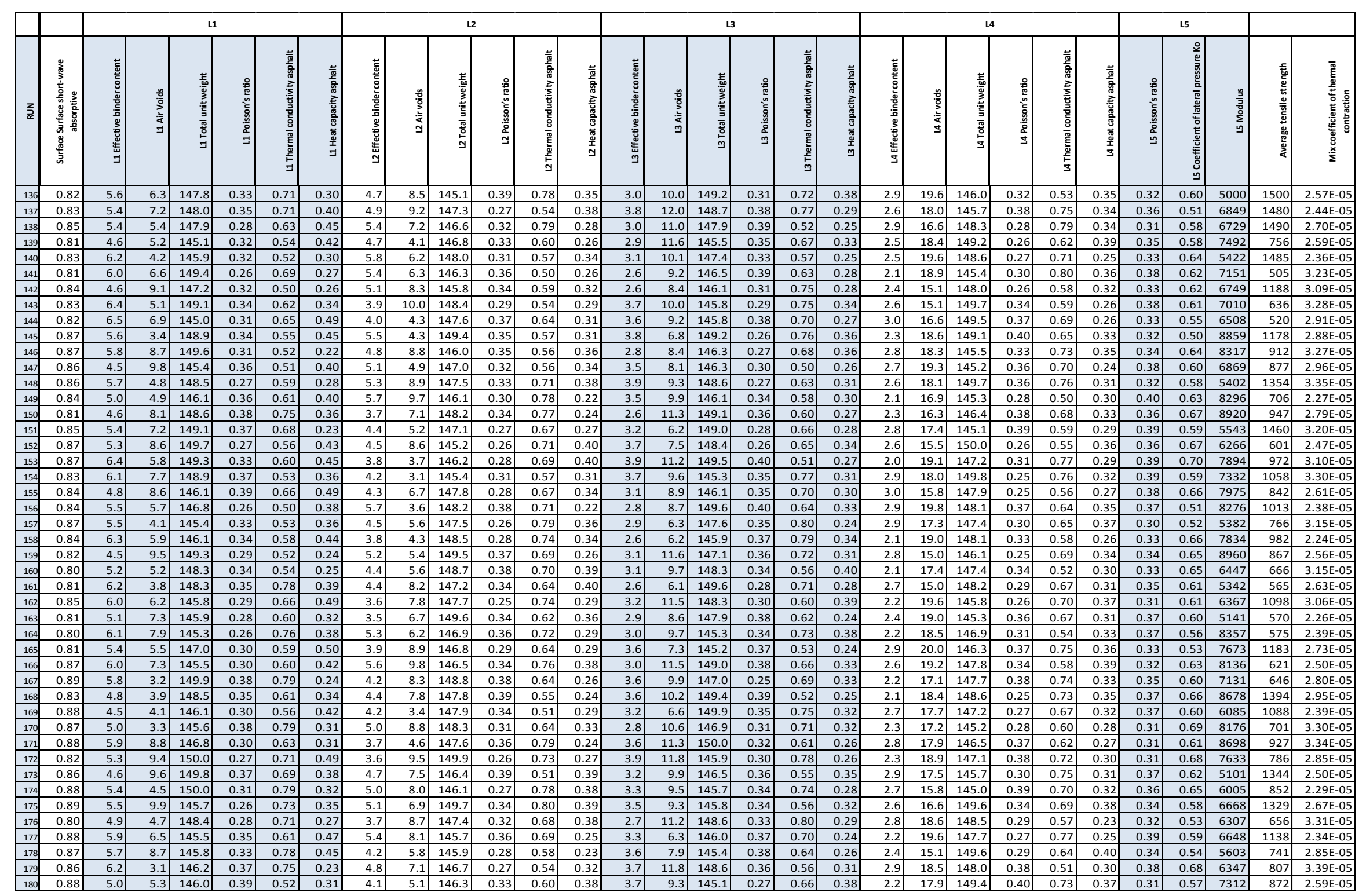




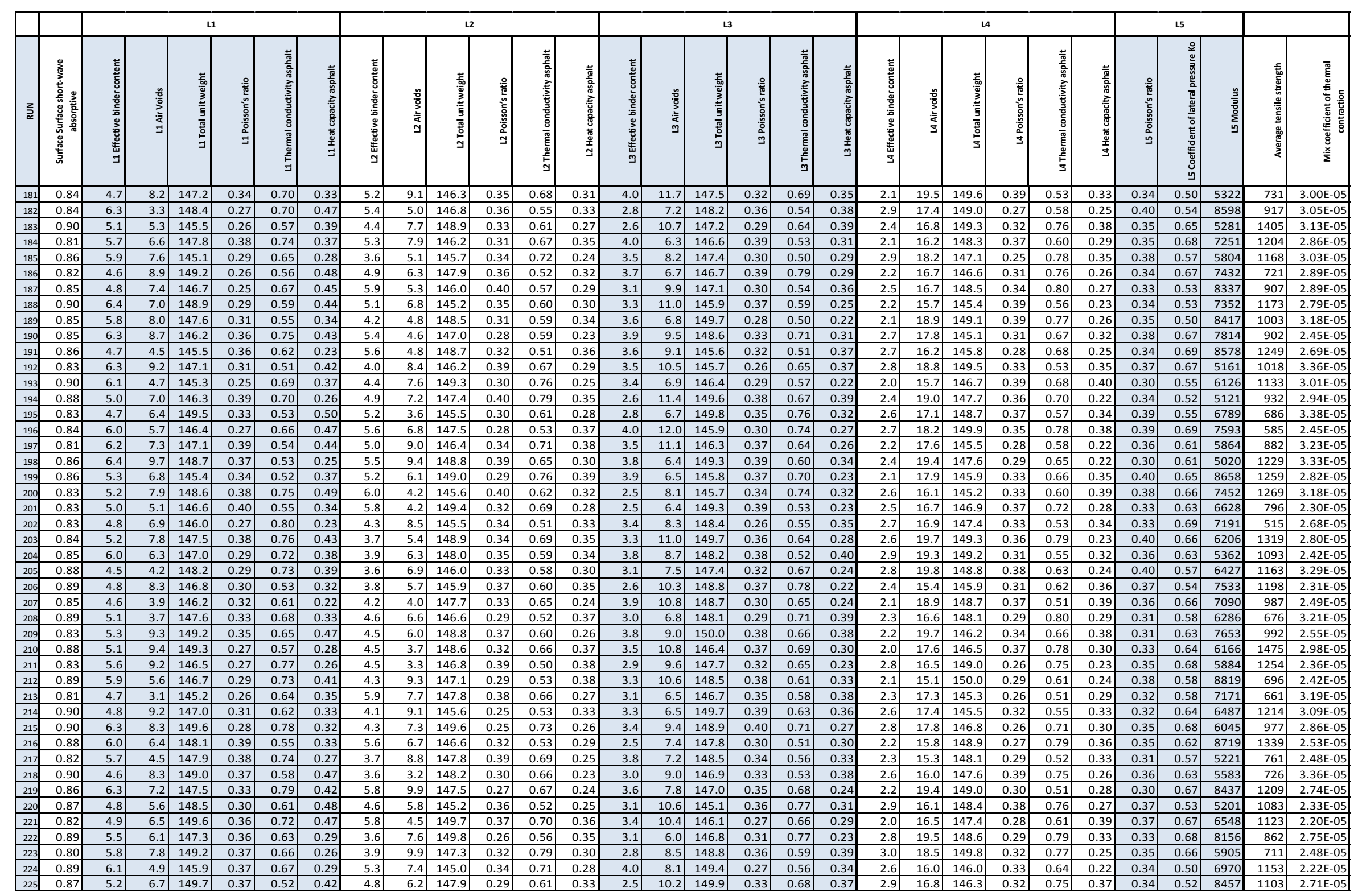




\begin{tabular}{|c|c|c|c|c|c|c|c|c|c|c|c|c|c|c|c|c|c|c|c|c|c|c|c|c|c|c|c|c|c|c|}
\hline & & \multicolumn{6}{|c|}{ L1 } & \multicolumn{6}{|c|}{12} & \multicolumn{6}{|c|}{ L3 } & \multicolumn{6}{|c|}{$\mathrm{L} 4$} & \multicolumn{3}{|c|}{ L5 } & \multirow{2}{*}{\multicolumn{2}{|c|}{ 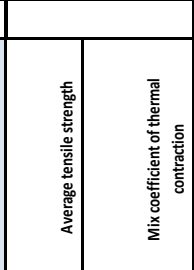 }} \\
\hline że & 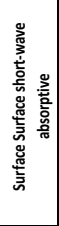 & 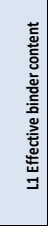 & 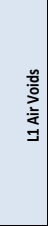 & 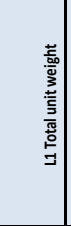 & 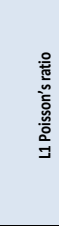 & 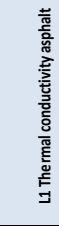 & 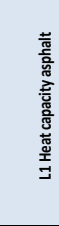 & 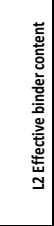 & 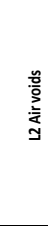 & 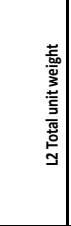 & 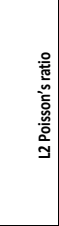 & 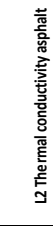 & 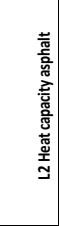 & 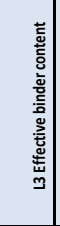 & 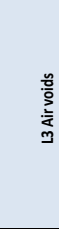 & 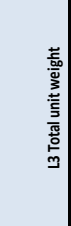 & 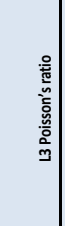 & 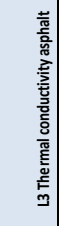 & 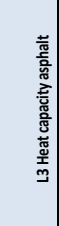 & 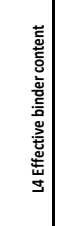 & 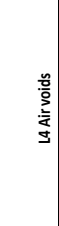 & 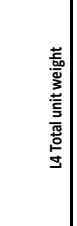 & 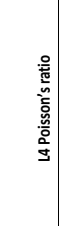 & 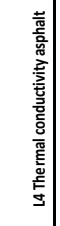 & 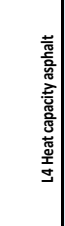 & 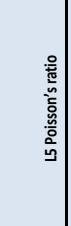 & 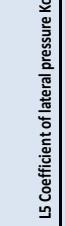 & 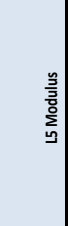 & & \\
\hline 226 & 0.88 & 5.6 & 6.2 & 148.0 & 0.35 & 0.63 & 0.46 & 4.6 & 6.9 & 149.5 & 0.38 & 0.68 & 0.23 & 3.9 & 11.8 & 146.4 & 0.32 & 0.64 & 0.36 & 2.3 & 15.6 & 146.5 & 0.34 & 0.52 & 0.25 & 0.34 & 0.69 & 8397 & 1359 & $2.37 \mathrm{E}-05$ \\
\hline & 0.84 & 5.8 & 6.9 & 145.6 & 0.39 & 0.54 & 0.26 & 4.7 & 7.0 & 145.7 & 0.30 & 0.56 & 0.39 & 2.8 & 8.0 & \begin{tabular}{|l|l|}
149.8 \\
\end{tabular} & 0.40 & 0.54 & 0.25 & 2.6 & 17.2 & 147.8 & 0.30 & 0.51 & 0.25 & 0.31 & 0.58 & 7935 & 922 & $2.66 \mathrm{E}-05$ \\
\hline & 0.82 & 5.2 & 9.5 & 148.8 & 0.35 & 0.79 & 0.44 & 4.4 & 3.9 & 148.4 & 0.27 & .77 & 0.27 & 3.4 & 6.6 & 147.9 & 0.33 & 0.73 & 0.35 & 2.1 & 17.1 & 147.0 & 0.27 & 0.71 & 0.36 & .39 & 0.60 & 0060 & 606 & $2.77 \mathrm{E}-05$ \\
\hline & 0.83 & 4.7 & 7.5 & 147.6 & 0.34 & 0.59 & 0.30 & 4.8 & 3.1 & 146.3 & 0.36 & 5.70 & 0.25 & 2.7 & 7.8 & 149.2 & 0.28 & 0.75 & 7 & 2.3 & \begin{tabular}{ll|}
19.4 \\
\end{tabular} & 147.0 & & .58 & & & & 940 & 1425 & \\
\hline 230 & 0.87 & 5.9 & 6.0 & \begin{tabular}{|l|}
147.7 \\
\end{tabular} & 0.39 & 0.75 & 0.38 & 5.3 & 6.8 & 149.7 & 0.39 & 0.72 & 0.28 & 3.8 & 9.3 & \begin{tabular}{|l|l|}
149.8 \\
\end{tabular} & 0.27 & 0.76 & 0.25 & 3.0 & 16.3 & 149.4 & 0.26 & 0.72 & 0.34 & 0.37 & 0.56 & 5744 & 967 & $2.75 \mathrm{E}-05$ \\
\hline 231 & 0.89 & 6.1 & 6.7 & \begin{tabular}{|l|}
146.4 \\
\end{tabular} & 0.34 & 0.51 & 0.27 & 3.6 & 4.4 & 149.2 & 0.34 & 0.54 & 0.32 & 3.0 & 11.7 & \begin{tabular}{|l|l|}
147.4 \\
\end{tabular} & 0.34 & 0.80 & 0.30 & 2.4 & 17.0 & 148.5 & 0.30 & 0.52 & 0.27 & 0.30 & 0.55 & 6588 & 847 & $2.83 \mathrm{E}-05$ \\
\hline 232 & 0.82 & 4.6 & 5.5 & 146.3 & 0.34 & 0.60 & 0.47 & 5.6 & 7.6 & \begin{tabular}{|l|}
147.1 \\
\end{tabular} & 0.26 & 0.77 & 0.33 & 3.1 & 11.1 & 145.0 & 0.34 & 0.68 & 0.31 & 3.0 & 19.3 & \begin{tabular}{|l|l|}
147.6 \\
\end{tabular} & 0.40 & 0.55 & 0.29 & 0.31 & $\begin{array}{l}0.70 \\
\end{array}$ & 7050 & 1063 & $3.10 \mathrm{E}-05$ \\
\hline 233 & 0.86 & $\begin{array}{ll}6.1 \\
\end{array}$ & 6.4 & 146.5 & 0.27 & 0.50 & 0.22 & 4.0 & 7.0 & 146.4 & 0.29 & 0.78 & 0.38 & \begin{tabular}{|l|l|}
3.9 \\
\end{tabular} & 8.3 & $\begin{array}{l}147.2 \\
\end{array}$ & \begin{tabular}{|l|l|}
0.27 \\
\end{tabular} & 0.55 & 0.22 & 2.1 & $\begin{array}{l}17.3 \\
\end{array}$ & \begin{tabular}{|c|}
145.6 \\
\end{tabular} & $\begin{array}{ll}0.35 \\
\end{array}$ & $\begin{array}{l}0.67 \\
\end{array}$ & 0.31 & 0.37 & \begin{tabular}{|l|l|}
0.54 \\
\end{tabular} & 5925 & \begin{tabular}{|c|}
1053 \\
\end{tabular} & $2.60 \mathrm{E}-05$ \\
\hline 234 & 0.85 & 5.6 & 3.8 & 149.2 & 0.32 & 0.68 & 0.25 & 3.6 & 4.2 & 150.0 & 0.33 & 0.64 & 0.25 & 3.0 & 6.1 & 146.6 & 0.34 & 0.73 & 0.35 & 2.5 & 16.9 & 146.2 & 0.31 & 0.52 & 0.35 & 0.36 & 0.62 & 8116 & 1008 & $2.90 \mathrm{E}-05$ \\
\hline 235 & 0.86 & 5.1 & 8.2 & 147.3 & 0.40 & 0.70 & 0.34 & 5.8 & 4.5 & 145.3 & & 7 & & 2.6 & 10.2 & 146.8 & 0.32 & 0.79 & & 2.5 & 15.4 & 149.7 & & 0.80 & & $x^{2}$ & & & & \\
\hline 236 & 0.89 & 5.7 & 9.6 & 146.0 & 0.30 & 0.57 & 0.31 & 5.7 & 7.0 & 146.5 & 0.30 & 0.68 & 0.31 & 2.6 & 10.7 & 147.0 & 0.29 & 0.55 & 0.26 & 2.2 & 17.5 & 149.9 & 0.32 & 0.61 & 0.22 & 0.36 & & 6990 & 716 & $3.06 \mathrm{E}-05$ \\
\hline 237 & 0.86 & 5.9 & 4.8 & 149.4 & 0.35 & 0.53 & 0.30 & 4.1 & 6.5 & 149.9 & 0.27 & 0.74 & 0.23 & 2.6 & 7.3 & \begin{tabular}{|l|l|}
149.1 \\
\end{tabular} & 0.27 & 0.66 & 0.34 & 2.9 & 18.1 & 147.3 & 0.27 & 0.60 & 0.31 & 0.38 & 0.55 & 5623 & 771 & $2.56 \mathrm{E}-05$ \\
\hline 238 & 0.80 & 4.9 & 5.4 & \begin{tabular}{|l|}
148.7 \\
\end{tabular} & 0.39 & 0.64 & 0.37 & 4.8 & 8.4 & 145.8 & 0.35 & 0.50 & 0.22 & 3.4 & 10.5 & 148.8 & 0.35 & 0.59 & 0.35 & 2.9 & 15.5 & 146.7 & 0.40 & 0.71 & 0.29 & 0.37 & 0.58 & 8779 & 500 & $2.76 \mathrm{E}-05$ \\
\hline 239 & 0.81 & 4.7 & 8.0 & 146.8 & 0.27 & 0.69 & 0.31 & 3.8 & 4.4 & 148.1 & 0.29 & 0.75 & 0.33 & 3.0 & 10.3 & 145.6 & 0.25 & 0.71 & 0.36 & 2.9 & 15.6 & 146.8 & 0.28 & 0.69 & 0.31 & 0.32 & 0.65 & 5784 & 1470 & $3.03 \mathrm{E}-05$ \\
\hline 240 & 0.85 & 5.2 & 4.2 & 146.9 & $\begin{array}{ll}0.38 \\
\end{array}$ & 0.55 & 0.43 & 4.6 & 2 & 149.5 & 0.25 & 0.66 & 0.39 & 2.9 & 11.5 & 145.5 & 0.25 & 0.56 & 0.35 & 2.9 & 18.2 & 148.2 & 0.36 & 0.67 & 0.31 & 0.40 & & 6327 & $\mid$\begin{tabular}{|l|l|}
1239 \\
\end{tabular} & $2.72 \mathrm{E}-05$ \\
\hline 241 & $\begin{array}{ll}0.87 \\
\end{array}$ & 4.8 & 7.4 & 147.5 & 0. & 0.60 & & 3 & & 149 & & & & 3.8 & 7.7 & 146.6 & & & & 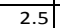 & 16 & & & & & & & & & $2.33 \mathrm{E}-05$ \\
\hline 242 & 0.84 & 5.9 & 6.2 & 148.6 & & & & & & 147.6 & & & & & 10.7 & & & & & 2.7 & & & & & & & & & & \\
\hline 243 & 0.80 & 5.5 & 4.0 & 149.1 & 0.26 & 0.69 & 0.31 & 4.7 & 4.0 & 149.9 & 0.26 & 0.56 & 0.27 & 4.0 & 6.2 & 147.6 & 0.27 & 0.62 & 0.25 & 2.7 & 19.4 & 146.6 & 0.31 & 0.73 & 0.27 & 0.36 & 0.62 & 7372 & 832 & $2.21 \mathrm{E}-05$ \\
\hline 244 & 0.80 & 5.2 & 5.6 & 146.6 & 0.29 & 0.72 & 0.29 & 4.9 & 8.6 & \begin{tabular}{|l|}
146.7 \\
\end{tabular} & 0.34 & 0.60 & 0.22 & 3.2 & 10.8 & 148.9 & 0.28 & 0.58 & 0.27 & 2.1 & 19.1 & 148.6 & 0.30 & 0.79 & 0.29 & 0.36 & 0.53 & 6769 & 535 & $2.34 \mathrm{E}-00$ \\
\hline 245 & 0.86 & 6.4 & 3.4 & 145.6 & 0.40 & 0.56 & 0.40 & 4.4 & 4.9 & \begin{tabular}{|l|}
149.1 \\
\end{tabular} & 0.29 & 0.68 & 0.28 & 3.9 & 8.8 & 148.8 & 0.28 & 0.68 & 0.26 & 2.3 & 18.8 & 145.0 & 0.36 & 0.66 & 0.40 & 0.35 & 0.57 & 5663 & 827 & $3.22 \mathrm{E}-05$ \\
\hline 246 & 0.85 & 5.3 & 8.0 & \begin{tabular}{|l|l|}
148.5 \\
\end{tabular} & 0.36 & 0.57 & 0.48 & 3.8 & 8.1 & 145.0 & 0.33 & 0.76 & 0.23 & 3.2 & 6.9 & 146.8 & 0.30 & 0.51 & 0.31 & 2.5 & 17.9 & 145.6 & & 0.57 & 0.28 & 0.37 & & 5523 & 1465 & $2.88 \mathrm{E}-05$ \\
\hline 247 & 0.82 & 5.6 & & 147.5 & 0.32 & 0.66 & & 4.9 & & 145 & & & & & 7.4 & & & & & 2.5 & & & & & & & & & 781 & $2.82 \mathrm{E}-05$ \\
\hline 248 & 0.85 & 4.8 & & & & & & & & & & & & & 7.6 & & & & & & & & & & & & & & & \\
\hline 249 & 0.89 & 4.7 & 8.2 & 145.2 & 0.4 & 0.80 & 0.3 & 4.5 & 8.0 & 146. & & 0.7 & & 3.1 & 8.1 & \begin{tabular}{|l|l|}
147.7 \\
\end{tabular} & & 0.7 & & 2.5 & 18.4 & 149.6 & & 0.79 & & 0.3 & & & 892 & $4 \mathrm{E}-05$ \\
\hline 250 & 0.88 & 5.9 & 3.2 & \begin{tabular}{|l|}
147.4 \\
\end{tabular} & 0.31 & 0.52 & 0.41 & 4.6 & 9.6 & 149.0 & 0.29 & 0.7 & 0. & 4.0 & 11.9 & $\begin{array}{l}149.5 \\
\end{array}$ & 0.33 & 0.58 & 0.40 & 2.4 & 19.9 & 148.2 & 0.3 & 0.66 & & 0.3 & 0.7 & & 957 & $2.94 \mathrm{E}-05$ \\
\hline 251 & 0.85 & 4.9 & 6.3 & \begin{tabular}{|l|l|}
146.6 \\
\end{tabular} & 0.29 & 0.51 & 0.3 & 4.7 & 3.8 & 149.3 & 0.3 & 0.75 & 0. & 3.4 & 10 & \begin{tabular}{|l|l|}
147.1 \\
\end{tabular} & & 0.62 & 0.27 & 2.6 & 15.7 & 147.1 & & 0.56 & 0.2 & 0.3 & & 52 & 746 & $2.83 \mathrm{E}-05$ \\
\hline 252 & 0.85 & 5.2 & 7.7 & 149.5 & 0.30 & 0.58 & 0.36 & 4.9 & 5.9 & 149.8 & & 0.77 & & 3.8 & 10 & 147 & & & & 2.7 & & & & 0.57 & & & & & 580 & $2.40 \mathrm{E}-05$ \\
\hline 253 & 0.86 & 5.3 & 3.6 & 148.4 & & 0.74 & & 5.9 & & 148 & & & & 2.9 & 11.1 & 148 & & & & 2.3 & & & & 0.62 & & & & & 1219 & $2.93 \mathrm{E}-05$ \\
\hline 254 & 0.84 & 5.5 & 8. & 147. & 0.2 & 0.54 & & & & 147 & & & & 39 & 9.8 & $14 !$ & & & & 2.4 & 16 & & & & & & & & & \\
\hline 255 & 0.90 & 6.2 & 8.8 & \begin{tabular}{|l|l|}
149.4 \\
\end{tabular} & 0.3 & 0.6 & & & & 147. & & & & 3. & 7.2 & $14 \varepsilon$ & & & & & 17 & & & & & & & & & \\
\hline 256 & 0.84 & 5.0 & 3.0 & 147.2 & 0.36 & 0.77 & 0.5 & 3.7 & 8.7 & 145.6 & & 0.7 & 0. & 3.2 & 9.8 & 149.1 & 0.3 & 0.72 & 0.2 & 2.0 & 18.8 & & & 0.61 & & & & & 751 & $4 \mathrm{E}-05$ \\
\hline 257 & 0.88 & 5.2 & 4.3 & \begin{tabular}{|l|}
146.4 \\
\end{tabular} & 0.35 & 0.50 & 0. & 5.1 & 7.5 & 146 & & & & & 6.6 & $14 \varepsilon$ & & 0. & & 3.0 & 19 & 14 & & 0.56 & & & & & & $3.02 \mathrm{E}-05$ \\
\hline 258 & 0.86 & 6.1 & 7.5 & \begin{tabular}{|l|l|}
147.1 \\
\end{tabular} & 0.34 & 0.80 & & 5.2 & 9.4 & 146 & & & & 3.4 & 6.2 & & & & & 2.4 & & & & 64 & & & & 90 & 837 & $2.52 \mathrm{E}-05$ \\
\hline 259 & 0.87 & 5.0 & 5.0 & \begin{tabular}{|l|l|}
146.7 \\
\end{tabular} & 0.34 & 0.80 & 0.45 & 6.0 & & \begin{tabular}{|l|}
149.4 \\
\end{tabular} & & 0.7 & 0.26 & 3.8 & 10.6 & 147 & 0.38 & 0. & 0.23 & 2.3 & 15 & 5.1 & & $\begin{array}{l}0.68 \\
\end{array}$ & 0.34 & 35 & & 8035 & \begin{tabular}{|l|l|}
1415 \\
\end{tabular} & $2.53 \mathrm{E}-05$ \\
\hline 260 & 0.85 & 6.2 & 3.9 & 149.5 & 0.31 & 0.63 & 0.4 & 5.0 & 6 & 145. & & 0.5 & & 3.5 & 7.6 & \begin{tabular}{|l|l|}
147.8 \\
\end{tabular} & 0.3 & 0.7 & 0.2 & 2.7 & 19.5 & 145.5 & & 0.56 & & 0.3 & 0.5 & 6407 & 611 & $2.64 \mathrm{E}-05$ \\
\hline & 0.82 & 6.3 & 4.3 & 148. & 0.39 & 0.7 & & & & 148. & & & & & 7. & $14 \mathrm{c}$ & & 0.5 & & & & & & & & & & & & \\
\hline 262 & 0.88 & 6.4 & 3.5 & 146.7 & 0.34 & 0.65 & 0. & 3.9 & & 147. & & 0.7 & & 2. & 6.0 & 145 & & & & & 19 & 147 & & & & & & & 128 & \\
\hline 263 & 0.89 & 5.2 & 9.8 & 149. & 0.3 & 0.7 & & 5. & & 149. & & & & & 8. & 145 & & & & & & & & & & & & & & \\
\hline 264 & 0.89 & 4.9 & 5.1 & 145 & & & & 4.9 & & 14 & & & & 2.9 & 9.6 & & & & & & & & & & & & & & 1143 & -05 \\
\hline 265 & 0.90 & 5.8 & 4.8 & 145.8 & 0.25 & 0.68 & 0.42 & 5.7 & 5.7 & 145 & & & & 3.2 & 10.5 & 148.1 & & & 0.3 & 2.1 & 17 & & & & & 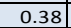 & 0. & & 651 & $3.21 \mathrm{E}-05$ \\
\hline 266 & 0.87 & 4.5 & 7.0 & \begin{tabular}{|l|l|}
145.7 \\
\end{tabular} & 0.31 & 0.64 & 0.33 & 4.5 & 7.9 & 149.1 & 0.25 & 0.62 & 0.25 & 2.9 & 7.3 & 148.9 & 0.27 & 0.64 & 0.27 & 2.2 & 16.1 & 146.8 & 0.32 & 0.62 & 0.38 & 0.38 & 0.70 & 6889 & 641 & 2.92E-05 \\
\hline & 0.83 & 4.6 & 4.6 & 149.5 & 0.28 & 0.75 & 0.25 & 5.5 & 9.0 & 148.4 & 0.31 & 0.52 & 0.23 & \begin{tabular}{|l|l|}
3.9 \\
\end{tabular} & 8.5 & 148.2 & 0.26 & 0.76 & 0.2 & 2.2 & 16.8 & 147.6 & 0.35 & 0.63 & 0.33 & 037 & 0.6 & 7754 & 1349 & $3.37 \mathrm{E}-05$ \\
\hline & 0. & 4 & & 149. & & 0.6 & & & & 145 & & & & & & 147 & & & & & & & & & & & & & & \\
\hline & 0.8 & 6.1 & 5.0 & & & 0.74 & & 4.5 & & & & 0.5 & & 2.7 & 9. & & 0.3 & 0.5 & & 2.8 & & & & & & & & & & \\
\hline & 0.88 & 5.4 & 5.5 & 49.1 & 0.29 & 0.64 & 0.40 & 3.7 & 4.5 & 149.5 & 0.32 & 0.55 & 0.37 & 2.7 & 8.5 & 146.2 & 0.31 & 0.73 & 0.24 & 2.1 & 15.5 & 146.7 & 0.38 & 0.61 & 0.39 & 0.38 & 0.53 & 5040 & 962 & $2.99 \mathrm{E}-05$ \\
\hline
\end{tabular}




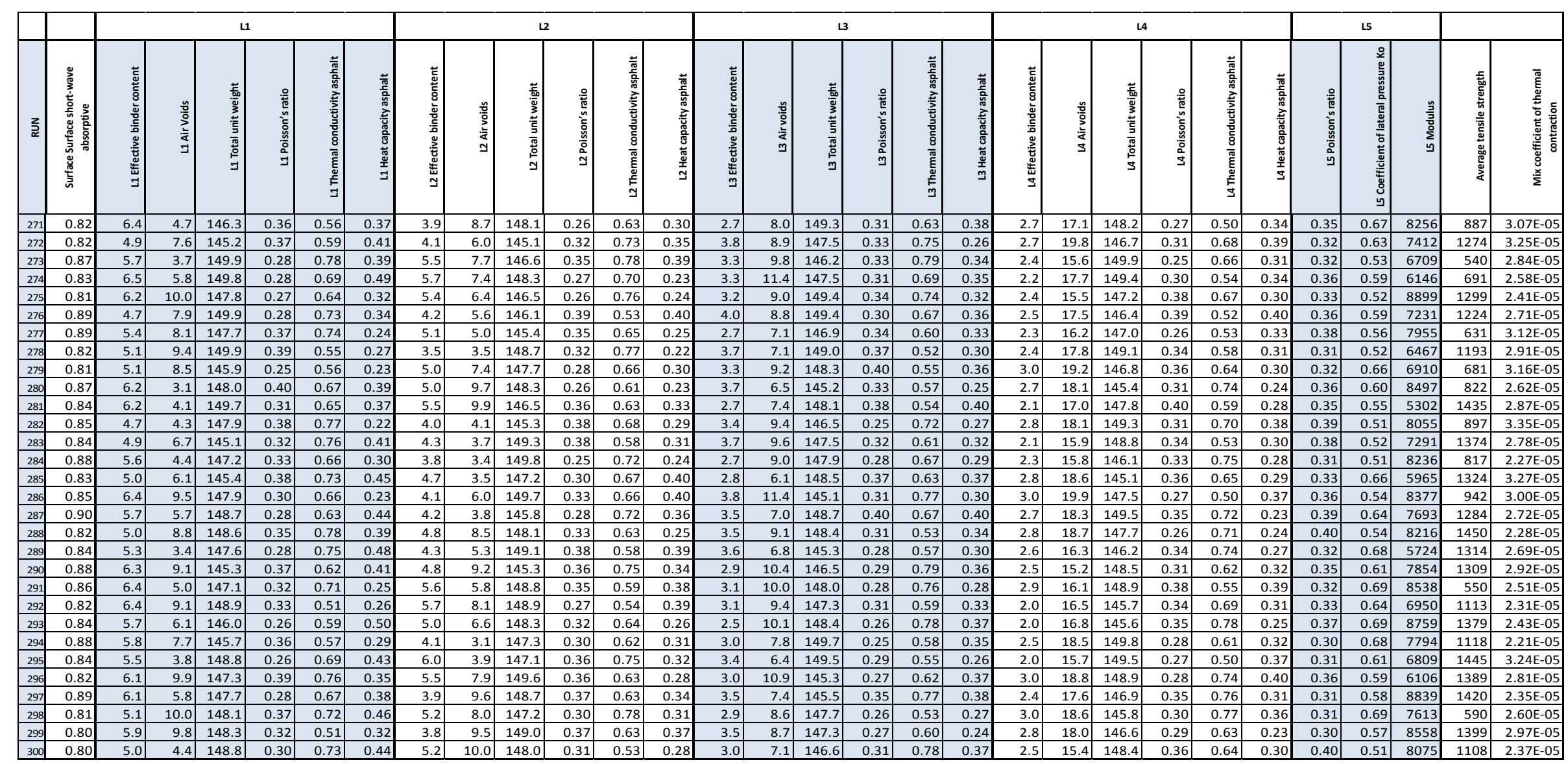


LHS for Materials Sentis tivity Structure 2

\begin{tabular}{|c|c|c|c|c|c|c|c|c|c|c|c|c|c|c|c|c|c|c|}
\hline \multirow[b]{2}{*}{$\underset{\widetilde{a}}{z}$} & \multirow[b]{2}{*}{ 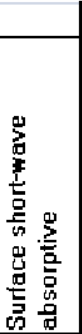 } & \multicolumn{6}{|c|}{ L1 } & \multicolumn{3}{|c|}{ L2 } & \multicolumn{3}{|c|}{ L3 } & \multicolumn{3}{|c|}{ L4 } & \multirow[b]{2}{*}{ 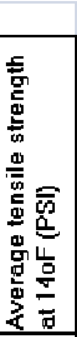 } & \multirow[b]{2}{*}{ 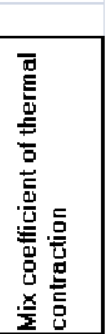 } \\
\hline & & 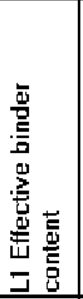 & $\begin{array}{l}\frac{n}{0} \\
\frac{3}{3} \\
\frac{5}{3} \\
\end{array}$ & 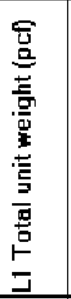 & 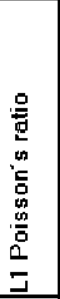 & 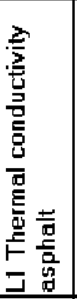 & 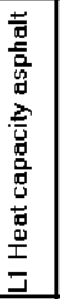 & 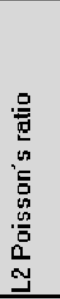 & 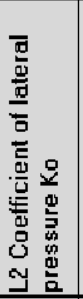 & 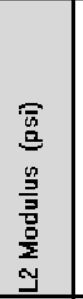 & 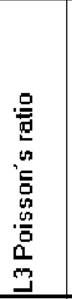 & 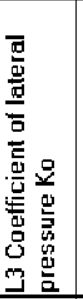 & 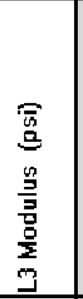 & 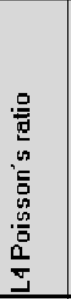 & 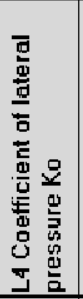 & 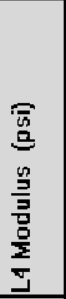 & & \\
\hline 1 & 0.83 & 4.7 & 8.5 & 148.2 & 0.28 & 0.76 & 0.31 & 0.25 & 0.56 & 28920 & 020 & 0.55 & 20377 & 0.32 & 0.66 & 9000 & 1329 & $2.50 \mathrm{E}-05$ \\
\hline 2 & 0.88 & 6.4 & 7.3 & 147.7 & 0.25 & 0.75 & 0.38 & 0.20 & 0.51 & 25553 & \begin{tabular}{l|l|}
0.19 \\
\end{tabular} & 0.60 & 18116 & 0.34 & 0.57 & 6990 & 1023 & $3.12 \mathrm{E}-05$ \\
\hline 3 & 0.90 & 5.0 & 3.4 & 148.9 & 0.39 & 0.67 & 0.48 & 0.18 & 0.57 & 28618 & 0.15 & 0.55 & 20779 & 0.33 & 0.56 & 6508 & 1193 & $3.33 E-05$ \\
\hline 4 & 0.82 & 4.8 & 3.1 & 145.4 & 0.27 & 0.65 & 0.24 & 0.20 & 0.59 & 27412 & 021 & 0.53 & 22387 & 0.32 & 0.54 & 7492 & 530 & 229E-05 \\
\hline 5 & 0.83 & 5.9 & 9.0 & 149.0 & 0.31 & 0.55 & 0.43 & 0.20 & 0.53 & 32990 & 0.17 & 0.50 & 19221 & 0.32 & 0.59 & 5141 & 1420 & $3.36 \mathrm{E}-05$ \\
\hline 6 & 0.86 & 5.1 & 6.3 & 146.9 & 0.38 & 0.52 & 0.28 & 0.24 & 0.54 & 32739 & 023 & 0.56 & 19824 & 0.38 & 0.63 & 5181 & 746 & $2.36 \mathrm{E}-05$ \\
\hline 7 & 0.90 & 5.8 & 8.9 & 149.3 & 0.38 & 0.70 & 0.43 & 0.24 & 0.55 & 34950 & 0.18 & 0.58 & 19322 & 0.37 & 0.65 & 7352 & 616 & 321E-05 \\
\hline 8 & 0.82 & 6.5 & 4.9 & 145.9 & 0.34 & 0.51 & 0.37 & 0.20 & 0.58 & 25754 & 020 & 0.52 & 15754 & 0.34 & 0.65 & 8337 & 997 & $2.64 \mathrm{E}-05$ \\
\hline 9 & 0.81 & 5.8 & 4.8 & 148.7 & 0.38 & 0.72 & 0.40 & 0.22 & 0.57 & 32638 & 025 & 0.54 & 22337 & 0.39 & 0.59 & 7995 & 1148 & $2.76 \mathrm{E}-05$ \\
\hline 10 & 0.83 & 5.3 & 4.4 & 147.2 & 0.37 & 0.53 & 0.37 & 0.23 & 0.52 & 25251 & 0.16 & 0.50 & 21985 & 0.36 & 0.52 & 7372 & 1349 & 294E-05 \\
\hline 11 & 0.89 & 5.8 & 5.4 & 147.6 & 0.34 & 0.68 & 0.26 & 0.22 & 0.58 & 25603 & 022 & 0.56 & 17462 & 0.39 & 0.67 & 8296 & 1379 & 324E-05 \\
\hline 12 & 0.82 & 5.5 & 5.8 & 149.8 & 0.33 & \begin{tabular}{l|l|}
0.74 \\
\end{tabular} & 0.23 & 0.19 & 0.52 & 32940 & 024 & 0.56 & 23643 & 0.33 & 0.68 & 5482 & 1244 & $2.57 \mathrm{E}-05$ \\
\hline 13 & 0.86 & 6.3 & 9.7 & 146.1 & 0.34 & 0.70 & 0.24 & 0.24 & 0.57 & 27211 & 020 & 0.59 & 16759 & 0.40 & 0.56 & 6427 & 71 & $2.59 \mathrm{E}-05$ \\
\hline 14 & 0.86 & 6.3 & 72 & 146.5 & 0.39 & 0.58 & 0.43 & 0.17 & 0.53 & 33693 & 0.18 & 0.53 & 17764 & 0.33 & 0.69 & 8357 & 1093 & $325 E-05$ \\
\hline 15 & 0.81 & 5.1 & 4.4 & 146.8 & 0.35 & 0.58 & 0.26 & 0.25 & 0.59 & 33090 & 0.15 & 0.53 & 15050 & 0.33 & 0.61 & 7975 & 1405 & $329 \mathrm{E}-05$ \\
\hline 16 & 0.83 & 6.0 & 4.0 & 149.9 & 0.38 & 0.71 & 0.42 & 0.23 & 0.52 & 26055 & 0.16 & 0.55 & 17010 & 0.35 & 0.55 & 5884 & 1158 & $2.54 \mathrm{E}-05$ \\
\hline 17 & 0.86 & 4.9 & 3.3 & 149.4 & 0.29 & 0.62 & 0.31 & 0.24 & 0.54 & 31533 & 0.25 & 0.51 & 18618 & 0.38 & 0.65 & 7231 & 1033 & $3.05 E-05$ \\
\hline 18 & 0.81 & 5.7 & 6.9 & 148.3 & 0.27 & 0.74 & 0.35 & 0.17 & 0.55 & 26759 & 024 & 0.50 & 18518 & 0.40 & 0.57 & 5784 & 1389 & $3.34 \mathrm{E}-05$ \\
\hline 19 & 0.83 & 52 & 5.3 & 147.1 & 0.39 & 0.58 & 0.42 & 0.18 & 0.51 & 27613 & 023 & 0.51 & 22286 & 0.34 & 0.51 & 5020 & 827 & 2.47E-05 \\
\hline 20 & 0.89 & 62 & 4.1 & 147.8 & 0.31 & 0.64 & 0.39 & 0.17 & 0.57 & 25201 & 022 & 0.58 & 15603 & 0.36 & 0.56 & 8839 & 1163 & 2.44E-05 \\
\hline 21 & 0.83 & 5.0 & 5.4 & 146.3 & 0.32 & 0.55 & 0.33 & 0.25 & 0.54 & 27915 & 020 & 0.59 & 21281 & 0.39 & \begin{tabular}{|l|}
0.65 \\
\end{tabular} & 5503 & 1465 & $3.37 \mathrm{E}-05$ \\
\hline 22 & 0.85 & 6.3 & 7.8 & 145.4 & 0.27 & 0.62 & 0.45 & 0.16 & 0.55 & 29322 & 0.19 & 0.52 & 21080 & 0.38 & 0.62 & 5442 & 927 & $224 E-05$ \\
\hline 23 & 0.90 & 5.4 & 8.4 & 146.4 & 0.35 & 0.79 & 0.50 & 0.18 & 0.55 & 26809 & 021 & 0.57 & 17563 & 0.34 & 0.63 & 6005 & 656 & $2.60 \mathrm{E}-05$ \\
\hline 24 & 0.89 & 4.9 & 72 & 147.8 & 0.31 & 0.71 & 0.46 & 0.17 & 0.57 & 35000 & 022 & 0.59 & 24045 & 0.37 & 0.59 & 7935 & 766 & $2.63 \mathrm{E}-05$ \\
\hline 25 & 0.83 & 6.1 & 8.7 & 145.9 & 0.36 & 0.63 & 0.50 & 0.18 & 0.56 & 26256 & 0.18 & 0.59 & 16960 & 0.35 & 0.60 & 5241 & 1229 & $3.14 \mathrm{E}-05$ \\
\hline 26 & 0.83 & 6.1 & 9.5 & 148.2 & 0.26 & 0.59 & 0.48 & 0.20 & 0.51 & 30327 & 0.19 & 0.57 & 15804 & 0.37 & 0.69 & 8799 & 1219 & $2.68 \mathrm{E}-05$ \\
\hline 27 & 0.87 & 5.7 & 4.1 & 147.1 & 0.28 & 0.54 & 0.32 & 0.18 & 0.58 & 32839 & 025 & 0.51 & 20980 & 0.30 & 0.53 & 6487 & 1500 & $3.00 \mathrm{E}-05$ \\
\hline 28 & 0.81 & 6.5 & 6.8 & 149.5 & 0.27 & 0.59 & 0.33 & 0.18 & 0.51 & 30779 & 0.17 & 0.57 & \begin{tabular}{|l|l|}
18367 \\
\end{tabular} & 0.32 & 0.67 & 5101 & 706 & $329 \mathrm{E}-05$ \\
\hline 29 & 0.84 & 6.5 & 3.7 & 148.8 & 0.35 & 0.79 & 0.31 & 0.20 & 0.60 & 26960 & 020 & 0.54 & 20226 & 0.32 & 0.55 & 6166 & 1319 & $3.19 \mathrm{E}-05$ \\
\hline 30 & 0.87 & 5.0 & 52 & 147.2 & 0.40 & 0.70 & 0.47 & 0.23 & 0.52 & 30477 & 022 & 0.54 & 15251 & 0.38 & 0.63 & 6467 & 912 & $3.03 E-05$ \\
\hline 31 & 0.88 & 5.6 & 5.9 & 146.8 & 0.29 & 0.76 & 0.41 & 0.19 & 0.59 & 30276 & 023 & 0.60 & 17613 & 0.32 & 0.56 & 5905 & 1470 & $3.07 \mathrm{E}-05$ \\
\hline 32 & 0.87 & 4.9 & 7.5 & 148.9 & 0.30 & 0.63 & 0.36 & 0.21 & 0.59 & 32085 & 024 & 0.58 & 18467 & 0.31 & 0.69 & 6688 & 545 & $2.66 \mathrm{E}-05$ \\
\hline 33 & 0.90 & 5.6 & 4.5 & 145.3 & 0.26 & 0.50 & 0.41 & 0.18 & 0.52 & 31935 & 0.19 & 0.57 & 19020 & 0.34 & 0.57 & 7774 & 631 & 2.45E-05 \\
\hline 34 & 0.83 & 4.8 & 7.5 & 147.8 & 0.36 & 0.53 & 0.31 & 0.15 & 0.57 & 34598 & 025 & 0.51 & 24146 & 0.33 & 0.66 & 8075 & 1173 & $293 \mathrm{E}-05$ \\
\hline 35 & 0.85 & 5.6 & 6.7 & 148.0 & 0.31 & 0.60 & 0.44 & 0.24 & 0.52 & 29623 & 022 & 0.58 & 18166 & 0.37 & 0.54 & 8136 & 505 & $224 E-05$ \\
\hline 36 & 0.81 & 4.7 & 3.1 & 146.3 & 0.30 & 0.59 & 0.32 & 0.19 & 0.51 & 32286 & 0.17 & 0.59 & 24749 & 0.34 & 0.53 & 7191 & 1133 & $3.04 \mathrm{E}-05$ \\
\hline 37 & 0.81 & 4.8 & 6.7 & 147.3 & 0.37 & 0.70 & 0.36 & 0.21 & 0.51 & 34799 & 025 & 0.54 & 18668 & 0.36 & 0.61 & 8759 & 666 & $3.38 E-05$ \\
\hline 38 & 0.80 & 62 & 7.4 & 148.7 & 0.32 & 0.56 & 0.30 & 0.15 & 0.57 & 33543 & 022 & 0.55 & 23794 & 0.34 & 0.58 & 6367 & 1254 & $222 \mathrm{E}-05$ \\
\hline 39 & 0.89 & 6.5 & 5.5 & 148.5 & 0.31 & 0.54 & 0.25 & 0.23 & 0.58 & 28417 & 0.18 & 0.52 & 20528 & $\begin{array}{l}0.32 \\
0.32\end{array}$ & 0.66 & 6186 & 1028 & $297 \mathrm{E}-05$ \\
\hline 40 & 0.87 & 5.1 & 9.5 & 148.3 & 0.27 & 0.55 & 0.25 & 0.19 & 0.55 & 26457 & 020 & 0.52 & 15000 & 0.33 & 0.69 & 7090 & 84 & $3.35 E-05$ \\
\hline 41 & 0.84 & & 5.8 & 146.2 & 0.34 & 0.77 & 0.27 & 0.21 & 0.57 & 26859 & 0.19 & 0.59 & 17362 & 0.31 & & 6106 & 1073 & 2.37E-05 \\
\hline 42 & 0.81 & 5.9 & 6.3 & 148.0 & 0.28 & 0.71 & 0.27 & 0.21 & 0.53 & 31030 & 0.15 & 0.50 & 24698 & 0.31 & 0.51 & 7573 & 982 & $298 E-05$ \\
\hline 43 & 0.90 & 6.0 & $\mid 7.4$ & 149.1 & $\begin{array}{l}0.20 \\
0.26\end{array}$ & 0.76 & 0.26 & 0.20 & $\begin{array}{l}0.55 \\
0.06\end{array}$ & 29724 & \begin{tabular}{|l|l|}
0.17 \\
\end{tabular} & 0.57 & 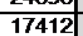 & \begin{tabular}{|l}
0.36 \\
0.06
\end{tabular} & $\begin{array}{l}0.70 \\
0.70\end{array}$ & 7332 & 540 & $2.62 \mathrm{E}-05$ \\
\hline
\end{tabular}




\begin{tabular}{|c|c|c|c|c|c|c|c|c|c|c|c|c|c|c|c|c|c|c|}
\hline & & \multicolumn{6}{|c|}{ L1 } & \multicolumn{3}{|c|}{ L2 } & \multicolumn{3}{|c|}{ L3 } & \multicolumn{3}{|c|}{ L4 } & \multirow[b]{2}{*}{ 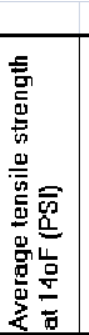 } & \multirow[b]{2}{*}{ 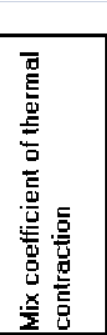 } \\
\hline$\underset{z}{\underline{z}}$ & 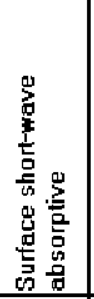 & 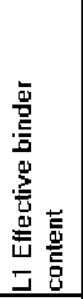 & $\begin{array}{l}\frac{0}{0} \\
\frac{5}{3} \\
\vdots \\
\vdots \\
\end{array}$ & 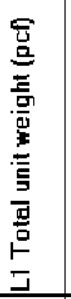 & 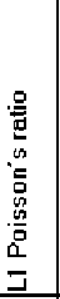 & 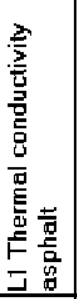 & 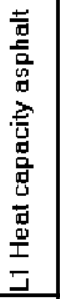 & 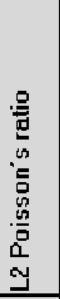 & 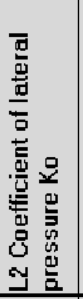 & 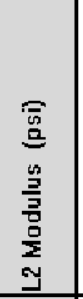 & 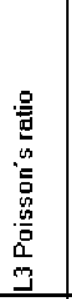 & 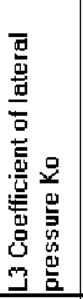 & 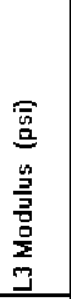 & 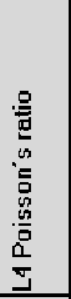 & 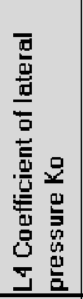 & 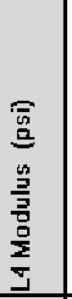 & & \\
\hline 44 & 0.83 & 5.6 & 8.3 & 146.8 & 026 & 0.73 & 0.43 & 0.15 & 0.58 & 26106 & 0.19 & 0.59 & 20276 & 0.38 & 0.68 & 7874 & 802 & $3.13 E-05$ \\
\hline 45 & 0.89 & 5.5 & 9.4 & 147.9 & 029 & 0.58 & 0.40 & 0.21 & 0.60 & 25050 & 0.15 & 0.58 & 16709 & 0.31 & 0.60 & 7714 & 701 & 2.92E-05 \\
\hline 46 & 0.87 & 5.9 & 7.4 & 149.0 & 0.30 & 0.51 & 0.46 & 0.17 & 0.54 & 29221 & 0.16 & 0.51 & 16357 & 0.30 & 0.57 & 8216 & 691 & 2.41E-05 \\
\hline 47 & 0.84 & 4.5 & \begin{tabular}{|l|}
9.3 \\
\end{tabular} & 146.5 & 027 & 0.59 & 0.32 & 0.19 & 0.58 & 31332 & 0.16 & 0.53 & 21382 & 0.39 & 0.62 & 8437 & 1123 & 3.01E-05 \\
\hline 48 & 0.89 & 5.9 & 8.8 & 145.5 & 0.36 & 0.63 & 0.35 & 0.16 & 0.51 & 28668 & 0.16 & 0.58 & 19975 & 0.38 & 0.53 & 5523 & 555 & $2.59 E-05$ \\
\hline 49 & 0.83 & 5.6 & 32 & \begin{tabular}{|l|}
149.1 \\
\end{tabular} & 027 & 0.78 & 0.36 & 0.21 & 0.52 & 31382 & 0.22 & 0.55 & 18417 & 0.32 & 0.58 & 7513 & 1450 & $2.66 \mathrm{E}-05$ \\
\hline 50 & 0.89 & 5.4 & 42 & \begin{tabular}{|l|}
148.6 \\
\end{tabular} & 026 & 0.52 & 0.26 & 0.19 & 0.58 & 33643 & 0.16 & 0.56 & 19372 & 0.37 & 0.53 & 5643 & 1038 & $2.85 E-05$ \\
\hline 51 & 0.87 & 6.0 & 5.8 & 145.7 & 0.33 & 0.79 & 0.34 & 0.21 & 0.54 & 33442 & 0.16 & \begin{tabular}{|l|}
0.53 \\
\end{tabular} & 16608 & 0.32 & 0.63 & 8678 & 1344 & $225 E-05$ \\
\hline 52 & 0.89 & 4.7 & $\begin{array}{l}9.6 \\
\end{array}$ & 1462 & 0.36 & 0.66 & 0.33 & 0.23 & 0.55 & 31080 & \begin{tabular}{l|l|}
0.17 \\
\end{tabular} & 0.60 & 16407 & 0.36 & 0.59 & 8417 & \begin{tabular}{|l|}
917 \\
\end{tabular} & $2.37 \mathrm{E}-05$ \\
\hline 53 & 0.84 & 6.1 & 8.0 & 149.9 & 0.34 & 0.50 & 0.44 & 0.20 & 0.58 & 29472 & 0.19 & 0.57 & 23141 & 0.36 & 0.51 & 8719 & 601 & $296 E-05$ \\
\hline 54 & 0.83 & 52 & 3.5 & \begin{tabular}{|l|}
145.4 \\
\end{tabular} & 0.40 & 0.71 & 0.34 & 0.18 & 0.55 & 32889 & 0.19 & 0.53 & 18819 & 0.34 & 0.68 & 6085 & 1440 & $2.74 E-05$ \\
\hline 55 & 0.80 & 5.1 & 3.6 & 146.9 & 028 & 0.79 & 0.49 & 0.19 & 0.56 & 32437 & 0.19 & 0.54 & 15905 & 0.32 & 0.08 & 6709 & \begin{tabular}{|l|}
947 \\
\end{tabular} & $2.95 E-05$ \\
\hline 56 & 0.89 & 4.6 & 9.1 & 147.7 & 0.32 & 0.59 & 0.30 & 0.16 & 0.52 & 32035 & 0.23 & 0.55 & 20025 & 0.34 & 0.51 & 5322 & 932 & $2.68 E-05$ \\
\hline 57 & 0.81 & 6.0 & 6.9 & \begin{tabular}{|l|}
145.3 \\
\end{tabular} & 0.35 & 0.71 & 0.42 & 0.22 & 0.59 & 25101 & 0.23 & 0.56 & 24095 & 0.31 & 0.51 & 7291 & 761 & $2.82 E-05$ \\
\hline 58 & 0.85 & 5.8 & 5.4 & 1482 & 0.33 & 0.77 & 0.38 & 0.21 & 0.60 & 26307 & 0.22 & 0.59 & 24447 & 0.36 & 0.67 & 5543 & 590 & $2.88 \mathrm{E}-05$ \\
\hline 59 & 0.87 & 6.5 & \begin{tabular}{l|l|}
3.4 \\
\end{tabular} & 147.5 & 0.39 & 0.66 & 029 & 0.20 & 0.55 & 30176 & $\begin{array}{l}0.16 \\
\end{array}$ & 0.53 & 23995 & 0.38 & 0.53 & 8156 & 887 & $2.30 E-05$ \\
\hline 60 & 0.84 & 6.4 & 5.5 & \begin{tabular}{|l|}
146.7 \\
\end{tabular} & 0.38 & 0.61 & 0.36 & 0.23 & 0.53 & 33040 & 0.21 & 0.54 & 21432 & 0.30 & 0.50 & 8196 & 1083 & $2.60 \mathrm{E}-05$ \\
\hline 61 & 0.83 & 5.8 & 6.1 & \begin{tabular}{|l|}
145.3 \\
\end{tabular} & 028 & 0.78 & 0.41 & 0.17 & 0.58 & 33141 & 0.25 & 0.57 & 18869 & 0.35 & 0.69 & 6045 & 535 & 221E-05 \\
\hline 62 & 0.84 & 52 & 6.7 & \begin{tabular}{|l|}
49.7 \\
\end{tabular} & 026 & 0.57 & 0.32 & 0.15 & 0.54 & 29171 & 0.22 & 0.55 & 24497 & 0.37 & 0.68 & 8819 & 1204 & $2.77 \mathrm{E}-05$ \\
\hline 63 & 0.81 & 6.4 & 5.0 & 145.9 & 029 & 0.80 & 0.39 & 0.23 & 0.54 & 30829 & 0.18 & 0.56 & 18065 & 0.37 & 0.54 & 7111 & 937 & 2.42E-05 \\
\hline 64 & 0.82 & 5.3 & \begin{tabular}{l|l|}
4.7 \\
\end{tabular} & \begin{tabular}{|l|}
149.6 \\
\end{tabular} & 0.36 & 0.71 & 0.45 & 0.15 & 0.53 & 34246 & 0.18 & 0.55 & 21181 & 0.3 & 0.66 & 7955 & 1198 & $228 \mathrm{E}-05$ \\
\hline 65 & 0.88 & 6.4 & 7.9 & 147.0 & 0.30 & 0.72 & 028 & 0.22 & 0.51 & 29874 & 0.24 & 0.51 & 20578 & 0.40 & 0.64 & 6910 & 882 & 2.48E-05 \\
\hline 66 & 0.88 & 5.0 & 6.1 & 149.1 & 0.37 & 0.69 & 0.44 & 0.20 & 0.56 & 32337 & 0.24 & 0.58 & 20879 & 0.31 & 0.68 & 8598 & 1480 & $3.03 E-05$ \\
\hline 67 & 0.88 & 4.9 & 5.6 & \begin{tabular}{|l|l|}
149.6 \\
\end{tabular} & 0.32 & 0.61 & 0.31 & 0.24 & 0.52 & 30578 & 0.21 & 0.58 & 16859 & 0.30 & 0.60 & 5583 & 1214 & $327 \mathrm{E}-05$ \\
\hline 68 & 0.85 & 6.1 & \begin{tabular}{ll|}
7.9 \\
\end{tabular} & \begin{tabular}{|l|}
145.7 \\
\end{tabular} & 0.34 & 0.68 & 0.37 & 0.21 & 0.60 & 27563 & 0.17 & 0.51 & 22739 & 0.32 & 0.55 & 8397 & 1259 & 3.39E-05 \\
\hline 69 & 0.82 & 5.4 & 8.9 & 148.5 & 0.37 & 0.80 & 0.40 & 0.24 & 0.55 & 34698 & 0.17 & 0.60 & 23241 & 0.3 & 0.55 & 5965 & 1384 & 2.82E-05 \\
\hline 70 & 0.85 & 4.9 & 8.3 & 148.9 & 026 & 0.53 & 0.49 & 0.17 & 0.54 & 32688 & 0.20 & 0.59 & 16307 & & 0.59 & 6769 & 1289 & 2.95E-05 \\
\hline 71 & 0.90 & 5.5 & \begin{tabular}{|l|}
9.3 \\
\end{tabular} & 148.5 & 0.39 & 0.61 & 0.32 & 0.24 & 0.56 & 29121 & 0.23 & 0.52 & 18920 & 0.35 & 0.51 & 6849 & 716 & $323 E-05$ \\
\hline 72 & 0.86 & 5.4 & \begin{tabular}{l|l|}
8.1 \\
\end{tabular} & 149.0 & 029 & 0.73 & 0.46 & 0.16 & 0.58 & 27111 & 0.16 & 0.57 & 23945 & 0.34 & 0.51 & 7653 & 1143 & $3.06 \mathrm{E}-05$ \\
\hline 73 & 0.88 & 5.9 & 8.4 & 149.9 & 0.35 & 0.62 & 022 & 0.16 & 0.56 & 30729 & 0.20 & 0.56 & 15653 & 0.38 & 0.57 & 7854 & 1183 & $227 \mathrm{E}-05$ \\
\hline 74 & 0.82 & 5.0 & \begin{tabular}{l|l}
5.6 \\
\end{tabular} & 149.5 & 027 & 0.56 & 0.47 & 0.24 & 0.55 & 26206 & 0.18 & 0.53 & 20477 & 0.36 & 0.69 & 6387 & 1168 & $2.77 \mathrm{E}-05$ \\
\hline 75 & 0.86 & 62 & 9.4 & 146.3 & 0.34 & 0.54 & 027 & 0.19 & 0.52 & 33945 & 0.19 & 0.55 & 19925 & & & 5824 & 1138 & $3.13 E-05$ \\
\hline 76 & 0.90 & 5.4 & 92 & 147.9 & 0.36 & 0.77 & 0.37 & 0.22 & 0.57 & 28467 & 0.19 & 0.55 & 23844 & 0.38 & 0.50 & 7312 & 1058 & $2.51 E-05$ \\
\hline 77 & 0.86 & 5.7 & 6.6 & 1452 & 025 & 0.65 & 0.30 & 0.18 & 0.59 & 26910 & 0.16 & 0.53 & 15201 & 0.34 & 0.52 & 7151 & 1048 & 2.43E-05 \\
\hline 78 & 0.88 & 5.4 & 9.6 & \begin{tabular}{|l|}
145.4 \\
\end{tabular} & 027 & 0.60 & 0.47 & 0.15 & 0.54 & 30528 & 0.22 & 0.57 & 22638 & 0.39 & 0.64 & 8176 & 1445 & 3.12E-05 \\
\hline 79 & 0.88 & 52 & 6.3 & \begin{tabular}{|l|}
146.7 \\
\end{tabular} & 0.39 & 0.80 & 0.33 & 0.16 & 0.59 & 29925 & 0.23 & 0.54 & 19171 & 0.40 & 0.5 & 6065 & 626 & $3.16 E-05$ \\
\hline 80 & 0.89 & 6.0 & 8.6 & 147.4 & 0.36 & 0.52 & 0.38 & 0.17 & 0.5 & 26005 & 0.23 & 0.58 & 19724 & & & 8879 & 676 & $3.09 \mathrm{E}-05$ \\
\hline 81 & 0.90 & 5.3 & 52 & 146.4 & 0.29 & 0.64 & 0.39 & 0.23 & 0.52 & 29070 & 0.20 & 0.54 & 23543 & 0.40 & 0.53 & 5161 & 721 & $2.86 \mathrm{E}-05$ \\
\hline 82 & 0.86 & 5.8 & 92 & \begin{tabular}{l|l|}
149.0 \\
\end{tabular} & 028 & 0.79 & 024 & 0.15 & 0.54 & 31583 & 0.21 & 0.59 & 20126 & 0.38 & 0.54 & 7211 & 580 & $323 E-05$ \\
\hline 83 & 0.89 & 4.6 & 8.3 & \begin{tabular}{|l|l|}
148.7 \\
\end{tabular} & 025 & 0.69 & 0.49 & 0.22 & 0.56 & 34447 & 0.21 & 0.52 & 20678 & 0.36 & 0.58 & 5844 & 1234 & 2.33E-05 \\
\hline 84 & 0.80 & & \begin{tabular}{l|l|}
4.7 \\
\end{tabular} & 145.0 & 0.34 & & & 0.18 & & 27663 & & & & & & 6568 & 897 & $220 \mathrm{E}-05$ \\
\hline 85 & 0.87 & 5.1 & 3.7 & 145.5 & 029 & 0.76 & 025 & 0.25 & 0.58 & 30025 & 0.22 & 0.51 & 19573 & 0.35 & 0.66 & 5060 & 942 & $2.98 E-05$ \\
\hline 86 & 0.88 & 4.8 & $\begin{array}{ll}3.6 \\
\end{array}$ & 148.6 & 029 & 0.53 & 0.34 & 0.21 & 0.54 & 34347 & 0.21 & 0.50 & 22085 & 0.31 & 0.69 & 6789 & 872 & $2.41 \mathrm{E}-05$ \\
\hline
\end{tabular}




\begin{tabular}{|c|c|c|c|c|c|c|c|c|c|c|c|c|c|c|c|c|c|c|}
\hline \multirow[b]{2}{*}{ Z } & \multirow[b]{2}{*}{ 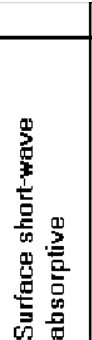 } & \multicolumn{6}{|c|}{ L1 } & \multicolumn{3}{|c|}{ L2 } & \multicolumn{3}{|c|}{$\mathbf{L 3}$} & \multicolumn{3}{|c|}{ L4 } & \multirow[b]{2}{*}{ 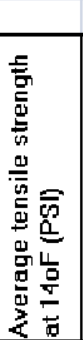 } & \multirow[b]{2}{*}{ 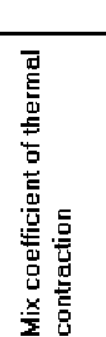 } \\
\hline & & 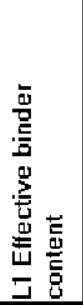 & $\begin{array}{l}\frac{n}{2} \\
\frac{D}{5} \\
= \\
= \\
=\end{array}$ & 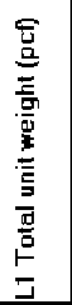 & 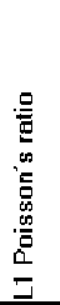 & 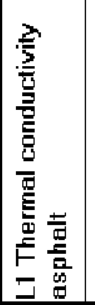 & 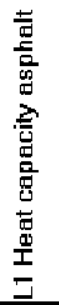 & 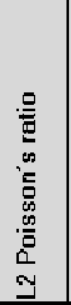 & 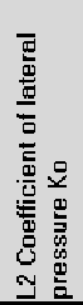 & 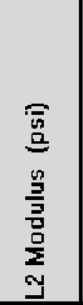 & 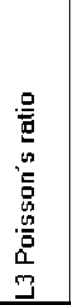 & 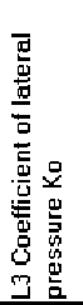 & 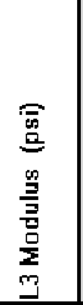 & 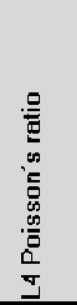 & 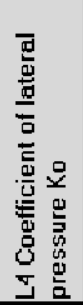 & 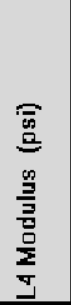 & & \\
\hline 87 & 0.85 & 5.0 & 4.6 & 145.5 & 0.28 & 0.60 & 0.35 & 0.21 & 0.52 & 31884 & 023 & 0.52 & 21332 & 0.39 & 0.51 & 7794 & 1209 & $226 \mathrm{E}-05$ \\
\hline 88 & 0.85 & 4.9 & 7.6 & 147.1 & 0.35 & 0.77 & 0.40 & 0.17 & 0.53 & 27513 & 0.17 & 0.53 & 25000 & 0.39 & 0.61 & 6548 & 1415 & $3.00 \mathrm{E}-05$ \\
\hline 89 & 0.86 & 6.1 & 42 & 149.5 & 0.30 & 0.78 & 0.45 & 0.24 & 0.59 & 34296 & 0.17 & 0.52 & 18216 & 0.37 & 0.59 & 7754 & 1013 & $2.73 E-05$ \\
\hline 90 & 0.89 & 5.1 & 7.5 & 145.1 & 0.33 & 0.61 & 0.40 & 0.23 & 0.54 & 26608 & 0.16 & 0.56 & 18719 & 0.38 & 0.69 & 7412 & 671 & $321 \mathrm{E}-05$ \\
\hline 91 & 0.85 & 5.3 & 6.6 & 149.3 & 0.26 & 0.73 & 0.46 & 0.19 & 0.54 & 30628 & 0.17 & 0.57 & 22889 & 0.38 & 0.68 & 5281 & 892 & $2.39 \mathrm{E}-05$ \\
\hline 92 & 0.83 & 6.3 & 32 & 146.6 & 0.31 & 0.78 & 0.42 & 0.21 & 0.58 & 30678 & 020 & 0.52 & 22186 & 0.33 & 0.65 & 6889 & 1490 & 2.34E-05 \\
\hline 93 & 0.85 & 5.6 & 3.3 & 147.3 & 0.38 & 0.50 & 0.28 & 0.24 & 0.51 & 33291 & 0.25 & 0.54 & 22789 & 0.37 & 0.57 & 8920 & 611 & $2.80 \mathrm{E}-05$ \\
\hline 94 & 0.80 & 4.7 & 7.8 & 145.8 & 0.30 & 0.69 & 0.37 & 0.16 & 0.50 & 28266 & 022 & 0.51 & 17513 & 0.33 & 0.55 & 8457 & 1098 & 2.71E-05 \\
\hline 95 & 0.83 & 4.6 & 3.1 & 148.4 & 0.29 & 0.68 & 0.29 & 0.22 & 0.59 & 34899 & 0.18 & 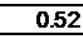 & 22437 & 0.38 & 0.66 & 8538 & 1304 & $2.39 \mathrm{E}-05$ \\
\hline 96 & 0.85 & 4.7 & 4.7 & 149.6 & 0.37 & 0.64 & 0.42 & 0.21 & 0.56 & 29422 & 0.16 & 0.56 & 21030 & 0.40 & 0.59 & 6950 & 510 & 2.67E-05 \\
\hline 97 & 0.89 & 4.8 & 5.5 & 1472 & 0.32 & 0.72 & 0.26 & 0.18 & 0.59 & 33794 & 0.20 & 0.56 & 18015 & 0.34 & 0.56 & 8618 & 832 & 3.35E-05 \\
\hline 98 & 0.87 & 6.3 & 5.6 & 149.4 & 0.38 & 0.67 & 0.47 & 0.18 & 0.59 & 30980 & 0.20 & 0.55 & 16106 & 0.32 & 0.56 & 6286 & 1399 & 2.31E-05 \\
\hline 99 & 0.85 & 6.4 & 5.3 & 1452 & 0.37 & 0.53 & 0.43 & 0.22 & 0.51 & 27161 & 0.23 & 0.56 & 21131 & 0.36 & 0.55 & 6266 & 560 & $3.38 \mathrm{E}-05$ \\
\hline 100 & 0.87 & 5.0 & 8.6 & 146.6 & 0.31 & 0.66 & 0.45 & 0.20 & 0.59 & 34146 & 0.23 & 0.51 & 21633 & 0.30 & 0.67 & 6025 & 1299 & 3.10E-05 \\
\hline 101 & 0.84 & 5.9 & 6.5 & 147.0 & 0.35 & 0.56 & 0.45 & 0.18 & 0.58 & 25503 & 0.17 & 0.54 & 24196 & 0.31 & 0.61 & 5221 & 585 & $2.69 \mathrm{E}-05$ \\
\hline 102 & 0.88 & 5.8 & 3.0 & 148.0 & 0.34 & 0.80 & 0.29 & 0.22 & 0.55 & 34548 & 0.20 & 0.58 & 19623 & 0.35 & 0.58 & 5945 & 1043 & $2.30 \mathrm{E}-05$ \\
\hline 103 & 0.83 & 5.4 & 6.6 & 1468 & 0.28 & 0.51 & 0.23 & 0.16 & 0.57 & 31131 & 023 & 0.59 & 21482 & 0.39 & 0.60 & 6628 & 621 & $290 \mathrm{E}-05$ \\
\hline 104 & 0.81 & 4.6 & 9.9 & 1492 & 0.30 & $\begin{array}{l}0.77 \\
\end{array}$ & 0.28 & 0.23 & 0.51 & 25352 & 0.18 & 0.56 & 19673 & 0.39 & 0.52 & 8518 & 967 & $2.70 \mathrm{E}-05$ \\
\hline 105 & 0.80 & 5.4 & 4.1 & 145.1 & 0.39 & 0.54 & 0.41 & 0.19 & 0.58 & 33492 & 0.23 & 0.51 & 17915 & 0.38 & 0.50 & 6930 & 1224 & $2.88 \mathrm{E}-05$ \\
\hline 106 & 0.85 & 6.4 & 3.0 & 146 & 0. & 0.62 & 0.44 & 0.24 & 0.56 & 27965 & 0.18 & & 23392 & 0.3 & 0.62 & 8035 & 1284 & $3.36 \mathrm{E}-05$ \\
\hline 107 & & & 5.7 & 145.7 & & 0.58 & 0.25 & 0.16 & 0.57 & 27462 & 0.19 & 5 & 20829 & 0.33 & 0.64 & 7070 & 1485 & $3.04 \mathrm{E}-05$ \\
\hline 108 & & 5.7 & 8.9 & 150 & 0. & 0.67 & 0.33 & 0.17 & 0.53 & 33392 & 0 & 0.51 & 19523 & 0.3 & 0.50 & 8497 & 1068 & 227E-05 \\
\hline 109 & 0.86 & 5.3 & 8.5 & 146.0 & 0.30 & 0.51 & 0.27 & 0.23 & 0.52 & 34497 & 0.22 & 0.53 & 24950 & 0.30 & 0.63 & 8779 & 796 & $2.91 \mathrm{E}-05$ \\
\hline 110 & 0.85 & 6.2 & 7.1 & 145.6 & 0.27 & 0.67 & 0.44 & 0.24 & 0.57 & 30075 & 0.1 & & 20930 & 0.35 & 0.63 & 5362 & 570 & $326 \mathrm{E}-05$ \\
\hline 111 & 0.86 & 5.2 & 3.8 & 146.9 & 0.34 & 0.64 & 0.33 & 0.16 & 0.50 & 33593 & 0.1 & 0.5 & 21533 & 0.37 & 0.63 & 6809 & 575 & $3.02 \mathrm{E}-05$ \\
\hline 112 & 0.82 & 5 & 4.6 & 147.9 & 0.33 & 0.65 & 0.50 & 0.22 & 0.51 & 29673 & 0.18 & & 23593 & 0.31 & 0.67 & 8055 & 781 & $3.40 \mathrm{E}-05$ \\
\hline 113 & 0.81 & 4.8 & 9.8 & 146.3 & 0.25 & 0.67 & 0.27 & 0.24 & 0.50 & 32789 & 0.16 & 0.54 & 23442 & 0.40 & 0.60 & 5864 & 857 & $2.62 \mathrm{E}-05$ \\
\hline 114 & 0.82 & 6 & 5.1 & 148.6 & 0.37 & 0.60 & 0.29 & 0.18 & 0.50 & 28367 & 022 & & 19070 & 0.36 & 0.6 & 7894 & 565 & 222E-05 \\
\hline 115 & 0.90 & 6. & 4.5 & 147.0 & 0. & 0.65 & 0.30 & 0.17 & 0.56 & 31482 & 0.24 & & 22588 & 0.33 & 0.59 & 5925 & 812 & $2.58 \mathrm{E}-05$ \\
\hline 116 & 0. & & 82 & 147.5 & & 0.64 & 0.22 & 0.22 & 0.54 & 25000 & 0. & & 19774 & 0.32 & 0.64 & 7673 & 651 & $2.99 \mathrm{E}-05$ \\
\hline 117 & 0.87 & 5. & 42 & 147.4 & 0.32 & 0.74 & 0.37 & 0.20 & 0.50 & 28819 & 024 & 0.60 & 20427 & 0.38 & 0.63 & 5121 & 550 & 2.42E-05 \\
\hline 118 & 0.89 & 5 & 4.3 & 148.1 & 0.33 & 0.54 & 0.31 & 0.25 & 0.50 & 31985 & 0. & 0.52 & 16055 & 0.35 & 0.53 & 8377 & 877 & 3.10E-05 \\
\hline 119 & & & & 148.7 & & & & 0. & 0.56 & 33995 & & & 15854 & 0.37 & & 5663 & 1334 & $E-05$ \\
\hline 120 & & & & 147 & & & & & 0. & 29020 & & & 18769 & 0.3 & 0.5 & 8638 & 837 & $3.01 \mathrm{E}-05$ \\
\hline 121 & 0.88 & 6.2 & 82 & 148.6 & 0. & 0.6 & 0. & 0.21 & 0.56 & 34648 & 0. & & 21683 & 0.31 & 0.62 & 6347 & 1455 & $2.56 \mathrm{E}-05$ \\
\hline 122 & & 4 & 6.8 & 1492 & & 0.6 & & 0.19 & 0.53 & 32236 & & & 20729 & 0.3 & 0.62 & 8558 & 1359 & 3.33E-05 \\
\hline 123 & 0.8 & 5. & 10.0 & 146.0 & 0. & 0.53 & 0.33 & 0.24 & 0.57 & 25653 & 021 & 0.54 & 24849 & 0.39 & 0.64 & 6729 & 1410 & 2.69E-05 \\
\hline 124 & & & & 148.3 & & & & 0.16 & 0.54 & 29824 & & & 17161 & & & 8899 & 1113 & $3.30 \mathrm{E}-05$ \\
\hline 125 & 0.84 & 5. & 4.3 & 145.1 & 0.31 & 0.77 & 0.29 & 0.17 & 0.52 & 27010 & 0.17 & 0.58 & 21231 & 0.32 & 0.58 & 6407 & 1178 & 223E-05 \\
\hline 126 & & & & 145.6 & & & & 0.25 & 0.56 & 25452 & & & 15352 & & & 8859 & 1088 & $2.79 \mathrm{E}-05$ \\
\hline 127 & 0.88 & 4.7 & 8.0 & 149.5 & 0.26 & 0.63 & 0.39 & 0.24 & 0.53 & 26658 & 0.16 & 0.51 & 23040 & 0.31 & 0.54 & 5201 & 1118 & 3.18E-05 \\
\hline 128 & & & 3.8 & 1482 & & & 0.48 & 0.16 & 0.51 & 33241 & 0.17 & & 22688 & 0.36 & 0.54 & 5302 & 977 & 3.32E-05 \\
\hline 129 & 0.84 & 4.6 & 4.4 & 147.0 & 0.35 & 0.78 & 0.25 & 0.20 & 0.58 & 27864 & 0.25 & 0.54 & 24548 & 0.33 & 0.62 & 7251 & 1103 & $3.30 \mathrm{E}-05$ \\
\hline
\end{tabular}




\begin{tabular}{|c|c|c|c|c|c|c|c|c|c|c|c|c|c|c|c|c|c|c|}
\hline & & \multicolumn{6}{|c|}{ L1 } & \multicolumn{3}{|c|}{ L2 } & \multicolumn{3}{|c|}{ L3 } & \multicolumn{3}{|c|}{ L4 } & \multirow[b]{2}{*}{ 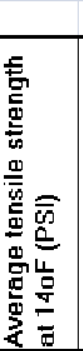 } & \multirow[b]{2}{*}{ 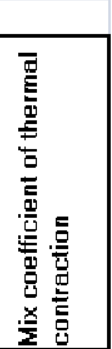 } \\
\hline z & 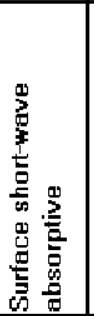 & 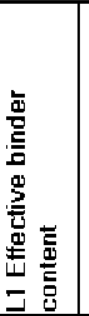 & 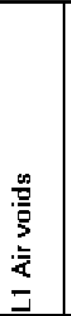 & 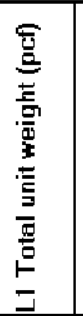 & 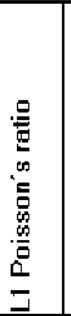 & 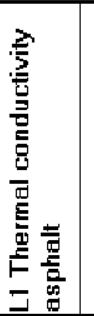 & 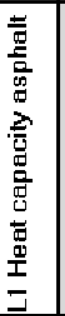 & 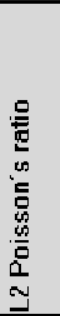 & 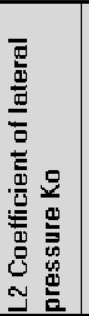 & 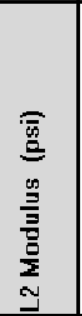 & 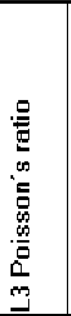 & 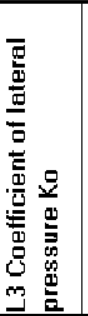 & 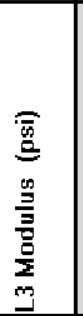 & 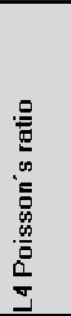 & 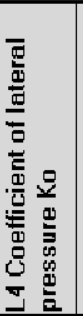 & 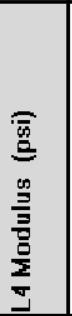 & & \\
\hline 130 & 0.88 & 62 & 5.7 & 146.4 & 0.29 & 0.73 & 0.38 & 0.25 & 0.56 & 32588 & 024 & 0.56 & 16005 & 0.33 & \begin{tabular}{c|}
0.58 \\
\end{tabular} & 8940 & 636 & 294E-05 \\
\hline 131 & 0.81 & 5.5 & 7.7 & 148.8 & 0.36 & 0.68 & 0.40 & 0.18 & 0.55 & 25151 & 024 & 0.57 & 15151 & 0.35 & 0.52 & 6246 & 606 & 297E-05 \\
\hline 132 & 0.87 & 6.1 & 5.3 & 147.9 & 027 & 0.59 & 0.49 & 0.21 & 0.54 & 27714 & 024 & 0.57 & 19271 & 0.37 & \begin{tabular}{|l|}
0.67 \\
\end{tabular} & 5402 & 1269 & 271E-05 \\
\hline 133 & 0.89 & 5.7 & 7.0 & 149.7 & 0.34 & 0.55 & 0.34 & 0.22 & 0.51 & 25704 & 021 & 0.56 & 23744 & 0.33 & 0.60 & 7915 & 1364 & 265E-05 \\
\hline 134 & 0.84 & 5.3 & 6.4 & 148.4 & 0.40 & 0.78 & 0.37 & 0.22 & 0.55 & 28317 & 0.18 & 0.51 & 23291 & 0.31 & 0.66 & 8256 & 681 & 235E-05 \\
\hline 135 & 0.86 & 5.6 & 3.9 & 149.7 & 0.31 & 0.75 & 0.22 & 0.16 & 0.55 & 28216 & 0.17 & 0.60 & 23693 & 0.32 & 0.65 & 6608 & 1264 & $3.09 \mathrm{E}-05$ \\
\hline 136 & 0.80 & 5.5 & 6.4 & 148.8 & 025 & 0.63 & 0.23 & 0.22 & 0.59 & 31784 & 0.18 & 0.54 & 20075 & 0.34 & 0.68 & 7392 & 520 & 3.17E-05 \\
\hline 137 & 0.86 & 5.5 & 8.0 & 146.5 & 0.37 & 0.60 & 0.23 & 0.17 & 0.56 & 33894 & 0.15 & 0.51 & 17261 & 0.34 & 0.58 & 7010 & 807 & 289E-05 \\
\hline 138 & 0.85 & 5.1 & 3.8 & 145.0 & 028 & 0.67 & 0.32 & 0.19 & 0.55 & 28065 & 021 & & 17211 & 0.40 & 0.51 & 6970 & 1108 & 3.15E-05 \\
\hline 139 & 0.81 & 6.0 & 5.1 & 1492 & 0.27 & 0.66 & 0.44 & 0.23 & 0.56 & 29372 & 0.18 & 0.6 & 15704 & 0.38 & 0.57 & 7472 & 862 & $327 \mathrm{E}-05$ \\
\hline 140 & 089 & 4.8 & 6.4 & 149.4 & 029 & 0.66 & 0.38 & 0.19 & 0.52 & 25302 & 0.16 & & 17965 & 0.33 & 0.51 & 6226 & 902 & 252E-05 \\
\hline 141 & 0.88 & f & 4 & 146.7 & 029 & 0.56 & 0.28 & 0.15 & 0.53 & 26357 & & & 17060 & & 0.61 & 6528 & 1128 & 233E-05 \\
\hline 142 & 0.89 & & 7.0 & 149.8 & 0.39 & 0.55 & 0.31 & 0.21 & 0.50 & 30126 & 0.1 & & 15503 & 0.3 & & 5382 & 817 & $278 \mathrm{E}-0$ \\
\hline 143 & 0.88 & & 8. & 146.4 & 0.32 & 0.65 & 0.24 & 0.23 & 0.60 & 31834 & & & 19874 & 0.3 & & 8477 & & $236 \mathrm{E}-0$ \\
\hline 144 & 0.86 & a & 3.7 & 145.5 & 0.38 & 0.69 & 0.36 & 0.20 & 0.57 & 27261 & 022 & & 17814 & 0.37 & & 8578 & 661 & $232 E-0$ \\
\hline 145 & 0.84 & & 6.0 & 148.4 & 0.38 & & 0.27 & 0.22 & 0.57 & 28518 & & & 16809 & & 0.6 & 8698 & 641 & $322 \mathrm{E}-05$ \\
\hline 146 & 0.82 & & 9.6 & 147.8 & 0.30 & 0.72 & 0.45 & 0.20 & 53 & 32186 & 0.1 & & 5452 & & & 704 & 741 & $320 \mathrm{E}-05$ \\
\hline 147 & & & & 149.4 & 0.36 & & 0.26 & 0.1 & 57 & 28769 & & & 23894 & & & & 736 & $228 E-05$ \\
\hline 148 & 0.81 & & 4.9 & 146.7 & 0.28 & 0.64 & 0.28 & 0.17 & 57 & 31683 & 02 & & 22035 & 0.37 & & 563 & 1394 & $256 \mathrm{E}-05$ \\
\hline 149 & 0.86 & & 5. & 150.0 & 0.32 & & 0.46 & 0.16 & 0.55 & 30226 & 0.18 & & 17312 & 0.39 & 0.5 & 7452 & 1374 & $278 E-05$ \\
\hline 150 & 0.82 & 5 & 5.0 & 145.6 & 0.35 & 0.66 & 0.34 & 0.22 & 0.60 & 34749 & 0.18 & & 22940 & 0.36 & 0.70 & 8317 & 500 & 291E-05 \\
\hline 151 & 0.84 & & 5. & 148.4 & 0.31 & 0.7 & 0.48 & 0.15 & 0.54 & 27312 & 02 & & 21734 & 0.31 & 0.57 & 5261 & 1339 & 251E-0 \\
\hline 152 & 0.87 & & 6 & 145.1 & 0.27 & 0.7 & 022 & 0.22 & 53 & 30427 & & & 24347 & & & & 1354 & $286 \mathrm{E}-0$ \\
\hline 153 & 0.86 & & 9 & \begin{tabular}{|l|}
147.3 \\
\end{tabular} & 0.35 & 0.7 & 0.49 & 0.24 & 50 & 31231 & 0.1 & & 22538 & 0.34 & 0.5 & 5422 & 962 & 265E-0? \\
\hline 154 & 0.89 & & 3. & 146.5 & 0.40 & 0 & 0.38 & 0.2 & 0.56 & 26508 & 02 & & 24296 & & & 5603 & 791 & 245E-0 \\
\hline 155 & 0.87 & & 9. & 147.4 & 0.26 & & 0.41 & 0.24 & .54 & 31281 & 0.1 & & 19422 & 0.36 & 0.5 & 7050 & 1309 & $326 \mathrm{E}-05$ \\
\hline 156 & & & & 149.8 & 0.31 & & 0.44 & 0.1 & & 26709 & & & 16658 & & & & 053 & $275 E-0$ \\
\hline 157 & & & & 147.6 & 0.33 & & 0.27 & 0.1 & & 26156 & & & 18568 & & & 8960 & 1249 & $289 \mathrm{E}-0$ \\
\hline 158 & 0.83 & & 8 & 149.8 & 0.33 & 0.5 & 0.27 & 0.23 & 0.59 & 28970 & 021 & 0.5 & 17864 & 0.35 & & 7693 & 1460 & $255 \mathrm{E}-0$ \\
\hline 159 & 0.86 & & & 145.3 & 0.37 & & 0.30 & & & 25804 & & & 15402 & & & 6588 & 711 & $328 \mathrm{E}-0$ \\
\hline 160 & 0.82 & & 8 & 145.6 & 0.36 & 0.7 & 0.49 & 0.18 & 53 & 31181 & 0.24 & & 16558 & 0.39 & & 7834 & 972 & 292E-0 \\
\hline 161 & & & & 147.6 & 0.30 & & & & & 26558 & & & 20236 & & & 5000 & 867 & 244E-0 \\
\hline 162 & 0.82 & & 32 & 149.6 & 0.33 & & 0.34 & 0.23 & 59 & 34045 & 02 & & 19121 & 0.33 & & 5040 & 1188 & 261E-0 \\
\hline 163 & & & & 149.9 & 0.31 & & 0.36 & & & 29271 & & & 24899 & & & & 756 & $283 \mathrm{E}-0$ \\
\hline 164 & 0.8 & & & 148 & & & & & & 28568 & & & 20176 & & & 764 & 1003 & $3.06 \mathrm{E}-0$ \\
\hline 165 & & & & 146. & & & & & & 33342 & & & 21583 & & & 7734 & 1078 & 3.11E-0 \\
\hline 166 & & & & 145. & & & & & & 321 & & & 15302 & & & 6869 & 1279 & $272 \mathrm{E}-0$ \\
\hline 167 & & & 6.0 & 145.8 & 028 & 0.5 & 0.30 & 0.1 & 0.5 & 25402 & 02 & & 23191 & 0.31 & & 8236 & 852 & $263 \mathrm{E}-0$ \\
\hline 168 & & & 7.6 & 146.1 & & & & & & 33844 & & & 20327 & & & 7814 & 726 & $221 E-0$ \\
\hline 169 & & & 4.0 & 146.6 & 0.37 & 0.76 & 0.35 & 0.16 & 0.53 & 27764 & 0.18 & & 16508 & 0.39 & 0.58 & 5804 & 1369 & $283 E-0$ \\
\hline 170 & 0.85 & & 7.3 & & & & & & & 29573 & & & 22990 & & & 6829 & 1475 & $234 E-0$ \\
\hline 171 & 0.84 & 4.6 & 9.1 & 148.3 & 0.39 & 0.72 & 0.32 & 0.2 & 0.59 & 28166 & 0.19 & 0.54 & 18266 & 0.35 & 0.61 & 5080 & 1008 & 285E-0? \\
\hline 172 & 87 & & & 147.3 & 34 & 52 & .41 & .20 & 0.56 & 34849 & & 0.58 & 17663 & 0.39 & 0.60 & 8276 & 1435 & 231E-0 \\
\hline
\end{tabular}




\begin{tabular}{|c|c|c|c|c|c|c|c|c|c|c|c|c|c|c|c|c|c|c|}
\hline \multirow[b]{2}{*}{$\underset{\widetilde{a}}{z}$} & \multirow[b]{2}{*}{ 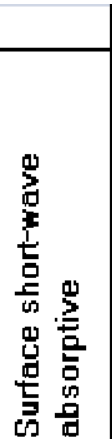 } & \multicolumn{6}{|c|}{ L1 } & \multicolumn{3}{|c|}{ L2 } & \multicolumn{3}{|c|}{$\mathbf{L 3}$} & \multicolumn{3}{|c|}{ L4 } & \multirow[b]{2}{*}{ 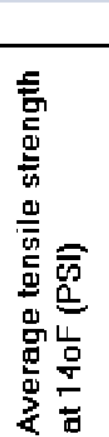 } & \multirow[b]{2}{*}{ 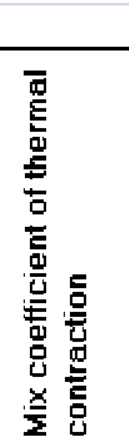 } \\
\hline & & 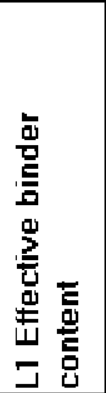 & 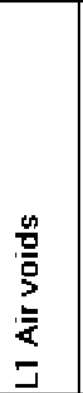 & 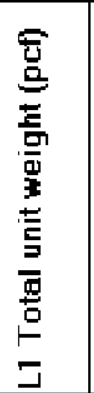 & 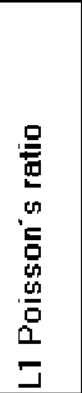 & 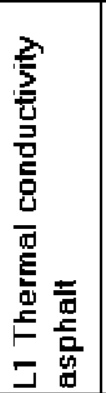 & 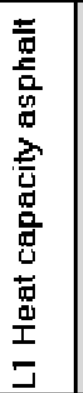 & 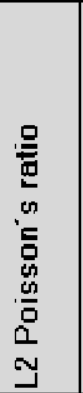 & 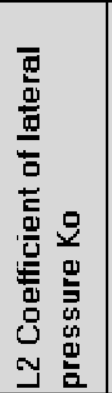 & 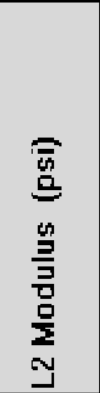 & 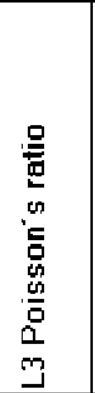 & 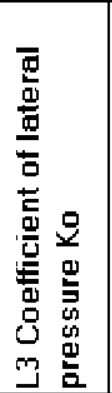 & 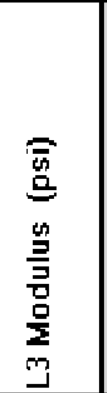 & 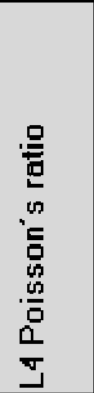 & 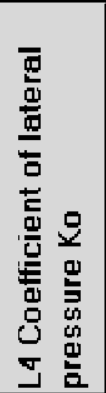 & 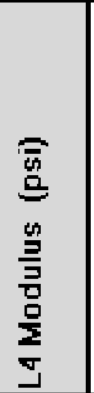 & & \\
\hline 173 & 0.90 & 62 & 4.8 & 149.3 & 028 & 0.76 & 0.42 & 0.18 & 0.55 & 29523 & 022 & 0.54 & 23492 & 0.40 & $0 . \overline{55}$ & 7553 & 1430 & $2.87 \mathrm{E}-05$ \\
\hline 174 & 0.86 & 4.6 & 4.5 & 148.8 & 0.32 & 0.74 & 0.45 & 0.23 & 0.59 & 28015 & 021 & 0.57 & 21834 & 0.32 & 0.52 & 7030 & 1018 & $2.49 E-05$ \\
\hline 175 & 0.80 & 6.4 & 6.1 & 149.7 & 0.35 & 0.73 & 0.46 & 0.17 & 0.58 & 33744 & 022 & 0.58 & 17111 & 0.34 & 0.55 & 6447 & 957 & $3.32 \mathrm{E}-05$ \\
\hline 176 & 0.83 & 5.6 & 9.9 & 147.7 & 0.35 & 0.77 & 0.39 & 0.16 & 0.58 & 28719 & 021 & 0.59 & 21935 & 0.33 & 0.65 & 7533 & 1495 & $238 \mathrm{E}-05$ \\
\hline 177 & 0.81 & 62 & 3.9 & 1462 & 0.33 & 0.75 & 028 & 0.23 & 0.51 & 31734 & 021 & 0.55 & 19472 & 0.31 & 0.65 & 7171 & 525 & $3.15 E-05$ \\
\hline 178 & 0.81 & 5.4 & 4.3 & 1452 & 0.36 & 0.56 & 0.30 & 0.25 & 0.51 & 26407 & 022 & 0.51 & 15101 & 0.33 & 0.58 & 6146 & 696 & $2.53 \mathrm{E}-05$ \\
\hline 179 & 0.84 & 6.0 & 9.1 & 148.9 & 0.38 & 0.61 & 0.50 & 0.16 & 0.50 & 31432 & 025 & 0.52 & 24246 & 0.35 & 0.66 & 7271 & 907 & $2.40 \mathrm{E}-05$ \\
\hline 180 & 0.87 & 5.5 & 3.5 & 146.9 & 0.40 & 0.62 & 0.49 & 0.17 & 0.58 & 27814 & 022 & 0.55 & 24397 & 0.38 & 0.67 & 7593 & 1294 & $3.08 \mathrm{E}-05$ \\
\hline 181 & 0.88 & 5.4 & 7.3 & 149.3 & 0.30 & 0.57 & 0.48 & 0.19 & 0.56 & 30930 & 023 & 0.50 & 23342 & 0.37 & 0.64 & 5724 & 751 & 3.31E-05 \\
\hline 182 & 0.84 & 5.0 & 72 & 147.4 & 0.38 & 0.70 & 0.46 & 0.19 & 0.52 & 30879 & 0.17 & 0.50 & 16156 & 0.31 & 0.52 & 6749 & 786 & 3.19E-05 \\
\hline 183 & 0.84 & 5.7 & 9.3 & 147.5 & 0.33 & 0.52 & 0.39 & 0.23 & 0.60 & 33191 & 0.17 & 0.56 & 16206 & 0.30 & 0.52 & 5683 & 987 & $2.75 E-05$ \\
\hline 184 & 0.86 & 4.9 & 6.0 & 145.9 & 0.40 & 0.55 & 0.38 & 0.22 & 0.52 & 25955 & 0.15 & 0.56 & 15955 & 0.35 & 0.63 & 5744 & 1153 & $2.48 \mathrm{E}-05$ \\
\hline 185 & 0.81 & 4.6 & 7.6 & 145.7 & 026 & 0.57 & 0.47 & 0.18 & 0.53 & 27060 & 025 & 0.57 & 22487 & 0.32 & 0.54 & 6327 & 686 & $2.57 \mathrm{E}-05$ \\
\hline 186 & 0.88 & 6.1 & 7.9 & 146.1 & 0.38 & 0.50 & 0.36 & 0.21 & 0.58 & 25905 & 024 & 0.56 & 15553 & 0.39 & 0.60 & 5462 & 1324 & $2.80 \mathrm{E}-0.5$ \\
\hline 187 & 0.88 & 4.7 & 4.8 & 147.5 & 0.30 & 0.70 & 024 & 0.18 & 0.52 & 30377 & 0.19 & 0.56 & 16457 & 0.38 & 0.69 & 8116 & 1274 & $225 \mathrm{E}-05$ \\
\hline 188 & 0.82 & 5.6 & 7.1 & 148.1 & 0.34 & 0.78 & 0.35 & 0.17 & 0.60 & 32487 & 0.19 & 0.52 & 23090 & 0.33 & 0.56 & 6668 & 515 & $3.07 \mathrm{E}-05$ \\
\hline 189 & 0.84 & 4.9 & 8.7 & 145.8 & 0.40 & 0.52 & 0.41 & 0.19 & 0.57 & 34095 & 021 & 0.57 & 16910 & 0.36 & 0.62 & 5985 & 776 & 3.39E-05 \\
\hline 190 & 0.82 & 5.3 & 8.1 & 147.7 & 0.36 & 0.67 & 0.47 & 0.16 & 0.57 & 28869 & 023 & 0.52 & 21784 & 0.38 & 0.56 & 8980 & 595 & $2.50 \mathrm{E}-05$ \\
\hline 191 & 0.85 & 6.3 & 7.7 & 1452 & 025 & 0.54 & 0.43 & 0.21 & 0.60 & 31633 & 024 & 0.55 & 20628 & 0.39 & 0.64 & 8095 & 847 & $2.81 E-05$ \\
\hline 192 & 0.82 & 6.3 & 10.0 & 1492 & 0.33 & 0.57 & 0.40 & 0.25 & 0.56 & 32387 & 023 & 0.51 & 17714 & 0.34 & 0.66 & 6648 & 822 & $2.47 \mathrm{E}-05$ \\
\hline 193 & 0.86 & 5.5 & 3.4 & 149.1 & 026 & 0.61 & 023 & 0.21 & 0.55 & 34196 & 024 & 0.59 & 22839 & 0.36 & 0.52 & 8658 & 992 & $2.72 \mathrm{E}-05$ \\
\hline 194 & 0.80 & 6.1 & 9.7 & 148.1 & 0.38 & 0.61 & 025 & 0.22 & 0.53 & 29774 & 0.16 & 0.53 & 18317 & 0.36 & 0.57 & 8015 & 1314 & $2.46 \mathrm{E}-05$ \\
\hline 195 & 0.85 & 4.8 & 62 & 146.0 & 0.30 & 0.51 & 0.48 & 0.20 & 0.60 & 25854 & 021 & 0.50 & 24648 & 0.36 & 0.60 & 7432 & 952 & $2.54 \mathrm{E}-05$ \\
\hline 196 & 0.83 & 4.8 & 5.7 & 1472 & 029 & 0.52 & 026 & 0.19 & 0.51 & 28116 & 020 & 0.53 & 16256 & 0.40 & 0.54 & 5623 & 731 & 3.18E-05 \\
\hline 197 & 0.81 & 5.0 & 9.8 & 147.1 & 0.37 & 0.58 & 0.43 & 0.19 & 0.52 & 32538 & 024 & 0.60 & 21884 & 0.35 & 0.61 & 6307 & 1425 & 2.84E-05 \\
\hline 198 & 0.88 & 5.8 & 3.5 & 148.0 & 0.36 & 0.57 & 0.36 & 0.24 & 0.54 & 34397 & 0.16 & 0.55 & 24598 & 0.36 & 0.69 & 6206 & 1239 & $2.53 E-05$ \\
\hline 199 & 0.83 & 5.5 & 9.5 & 146.1 & 026 & 0.72 & 029 & 0.15 & 0.53 & 29975 & 0.19 & 0.55 & 22136 & 0.31 & 0.63 & 5342 & 1063 & $3.16 E-05$ \\
\hline 200 & 0.89 & 4.7 & 7.0 & 146.0 & 025 & 0.71 & 0.48 & 0.19 & 0.50 & 27362 & 021 & 0.52 & 18970 & 0.35 & 0.58 & 7613 & 922 & $324 \mathrm{E}-05$ \\
\hline
\end{tabular}

John $\mathrm{H}$. 